\title{
Structure-based Molecular Networking for the Target Discovery of Oxahomoaporphine and 8-Oxohomoaporphine Alkaloids from Duguetia surinamensis
}

Weider H. P. Paz, ${ }^{\dagger} ₫$ Rodolfo N. de Oliveira,,${ }^{\ddagger}$ Gabriel Heerdt, ${ }^{\S}$ Célio F. F. Angolini, ${ }^{\perp}$ Lívia S. de Medeiros, $\|$ Valdenizia R. Silva, ${ }^{\nabla}$ Luciano S. Santos, ${ }^{\nabla}$ Milena B. P. Soares, ${ }^{\nabla}$ Daniel P. Bezerra ${ }^{\nabla}$ Nelson H. Morgon, ${ }^{\#}$ Jackson R.G.S. Almeida, ${ }^{\curvearrowleft}$ Felipe M. A. da Silva, ${ }^{*}$ Emmanoel V. Costa $^{*, \dagger}$ and Hector H. F. Koolen $*, \dagger$

$\dagger$ Metabolomics and Mass Spectrometry Research Group, Amazonas State University, Manaus 690065-130, Brazil

Department of Chemistry, Federal University of Amazonas, Manaus 69077-000, Brazil

$\S$ Department of Chemistry, Federal University of Minas Gerais, Belo Horizonte 31270-901, Brazil

${ }^{\perp}$ Center of Human and Natural Sciences, Federal University of ABC, 09210-580 Santo André, Brazil

\| Department of Chemistry, Federal University of São Paulo, 09920-540 Diadema, Brazil

${ }^{\nabla}$ Gonçalo Moniz Institute, Oswaldo Cruz Foundation, Salvador 40296-710, Brazil

\# Institute of Chemistry, University of Campinas, Campinas 13083-970, Brazil

I Center for Study and Research of Medicinal Plants, Federal University of Vale do São Francisco, Petrolina 56304-205, Brazil

\footnotetext{
ABSTRACT: In addition to seven known alkaloids (2, 6-11) and 1,2,4-trimethoxybenzene (1), three isoquinoline-derived alkaloids (3-5), namely, duguetinine (3), a compound based on an unprecedented oxahomoaporphine scaffold, and two new 8-oxohomoaporphine alkaloids, duguesuramine (4) and 11-methoxyduguesuramine (5), and a new asarone-derived phenylpropanoid (10) were isolated from the bark of Duguetia surinamensis. The isolation workflow was guided by HPLC-HRESI-MS/MS and molecular networking-based analyses. Twenty-four known alkaloids were dereplicated from the $D$. surinamensis alkaloid-rich fraction network and were assigned by manual MS/MS interpretation. Their cytotoxic potential was evaluated.
} 


\section{List of Supplementary Figures}

Figure S1. Total ion chromatogram of the alkaloid-rich fraction from D. surinamensis.

Figure S2. Chemical structures of the dereplicated compounds 2, 11, 12a-12h, 13a, 13b, 14a14n and 15.

Figure S3. Extracted ion chromatogram, MS and MS/MS spectra of compound 12a.

Figure S4. Extracted ion chromatogram, MS and MS/MS spectra of compound $\mathbf{1 2 b}$.

Figure S5. Extracted ion chromatogram, MS and MS/MS spectra of compound 12c.

Figure S6. Extracted ion chromatogram, MS and MS/MS spectra of compound 12d.

Figure S7. Extracted ion chromatogram, MS and MS/MS spectra of compound 12e.

Figure S8. Extracted ion chromatogram, MS and MS/MS spectra of compound $\mathbf{1 2 f}$.

Figure S9. Extracted ion chromatogram, MS and MS/MS spectra of compound 12g.

Figure S10. Extracted ion chromatogram, MS and MS/MS spectra of compound $\mathbf{1 2 h}$.

Figure S11. Extracted ion chromatogram, MS and MS/MS spectra of compound 13a.

Figure S12. Extracted ion chromatogram, MS and MS/MS spectra of compound $\mathbf{1 3 b}$.

Figure S13. Extracted ion chromatogram, MS and MS/MS spectra of compound 2.

Figure S14. Extracted ion chromatogram, MS and MS/MS spectra of compound 14a.

Figure S15. Extracted ion chromatogram, MS and MS/MS spectra of compound 14b.

Figure S16. Extracted ion chromatogram, MS and MS/MS spectra of compound 14c.

Figure S17. Extracted ion chromatogram, MS and MS/MS spectra of compound 14d.

Figure S18. Extracted ion chromatogram, MS and MS/MS spectra of compound 14e.

Figure S19. Extracted ion chromatogram, MS and MS/MS spectra of compound $\mathbf{1 4 f}$.

Figure S20. Extracted ion chromatogram, MS and MS/MS spectra of compound 14g.

Figure S21. Extracted ion chromatogram, MS and MS/MS spectra of compound $\mathbf{1 4 h}$.

Figure S22. Extracted ion chromatogram, MS and MS/MS spectra of compound 14i.

Figure S23. Extracted ion chromatogram, MS and MS/MS spectra of compound $\mathbf{1 4 j}$.

Figure S24. Extracted ion chromatogram, MS and MS/MS spectra of compound $\mathbf{1 4 k}$.

Figure S25. Extracted ion chromatogram, MS and MS/MS spectra of compound 141.

Figure S26. Extracted ion chromatogram, MS and MS/MS spectra of compound $\mathbf{1 4 m}$.

Figure S27. Extracted ion chromatogram, MS and MS/MS spectra of compound 15.

Figure S28. ${ }^{1} \mathrm{H}$ NMR spectrum of compound 3 in $\mathrm{CDCl}_{3}$ at $500 \mathrm{MHz}$. 
Figure S29. Enlargement ( $\delta 7.20-5.93)$ of ${ }^{1} \mathrm{H}$ NMR spectrum of compound $\mathbf{3}$ in $\mathrm{CDCl}_{3}$ at 500 $\mathrm{MHz}$.

Figure S30. Enlargement ( $\delta$ 4.72-3.93) of ${ }^{1} \mathrm{H}$ NMR spectrum of compound 3 in $\mathrm{CDCl}_{3}$ at 500 $\mathrm{MHz}$.

Figure S31. Enlargement ( $\delta 3.25-2.55)$ of ${ }^{1} \mathrm{H}$ NMR spectrum of compound 3 in $\mathrm{CDCl}_{3}$ at 500 $\mathrm{MHz}$.

Figure S32. Enlargement ( $\delta 3.25-2.97)$ of ${ }^{1} \mathrm{H}$ NMR spectrum of compound 3 in $\mathrm{CDCl}_{3}$ at 500 $\mathrm{MHz}$.

Figure S33. Enlargement $(\delta 2.73-2.65)$ of ${ }^{1} \mathrm{H}$ NMR spectrum of compound 3 in $\mathrm{CDCl}_{3}$ at 500 $\mathrm{MHz}$.

Figure 34. ${ }^{13} \mathrm{C}$ NMR spectrum of compound 3 in $\mathrm{CDCl}_{3}$ at $125 \mathrm{MHz}$.

Figure S35. Enlargement ( $\delta$ 148.8-100.9) of ${ }^{13} \mathrm{C}$ NMR spectrum of compound 3 in $\mathrm{CDCl}_{3}$ at $500 \mathrm{MHz}$.

Figure S36. Enlargement ( $\delta 66.7-29.4)$ of ${ }^{13} \mathrm{C}$ NMR spectrum of compound 3 in $\mathrm{CDCl}_{3}$ at 500 $\mathrm{MHz}$.

Figure S37. ${ }^{13} \mathrm{C}$ NMR DEP135 spectrum of compound 3 in $\mathrm{CDCl}_{3}$ at $125 \mathrm{MHz}$.

Figure S38. Enlargement $\left(\delta\right.$ 112.3-84.3) of ${ }^{13} \mathrm{C}$ NMR DEP135 spectrum of compound 3 in $\mathrm{CDCl}_{3}$ at $125 \mathrm{MHz}$.

Figure S39. Enlargement $(\delta 66.7-29.4)$ of ${ }^{13} \mathrm{C}$ NMR DEP135 spectrum of compound 3 in $\mathrm{CDCl}_{3}$ at $125 \mathrm{MHz}$.

Figure S40. ${ }^{1} \mathrm{H}-{ }^{1} \mathrm{H}$ COSY correlation map from ${ }^{1} \mathrm{H}$ NMR experiment of compound 3 in $\mathrm{CDCl}_{3}$ at $500 \mathrm{MHz}$.

Figure S41. Enlargement $\left(\delta\right.$ 4.72-3.83) of ${ }^{1} \mathrm{H}-{ }^{1} \mathrm{H}$ COSY correlation map from ${ }^{1} \mathrm{H}$ NMR experiment of compound 3 in $\mathrm{CDCl}_{3}$ at $500 \mathrm{MHz}$.

Figure S42. Enlargement ( $\delta$ 3.32-2.49) of ${ }^{1} \mathrm{H}-{ }^{1} \mathrm{H}$ COSY correlation map from ${ }^{1} \mathrm{H}$ NMR experiment of compound 3 in $\mathrm{CDCl}_{3}$ at $500 \mathrm{MHz}$.

Figure S43. ${ }^{1} \mathrm{H}_{-13}{ }^{13} \mathrm{C}$ one-bond correlation map from HSQC NMR experiment of compound 3 in $\mathrm{CDCl}_{3}$ at 500 and $125 \mathrm{MHz}$.

Figure S44. Enlargement $(\delta 7.20-5.93)$ of ${ }^{1} \mathrm{H}-{ }^{13} \mathrm{C}$ one-bond correlation map from HSQC NMR experiment of compound 3 in $\mathrm{CDCl}_{3}$ at 500 and $125 \mathrm{MHz}$.

Figure S45. Enlargement $(\delta 4.90-2.55)$ of ${ }^{1} \mathrm{H}-{ }^{13} \mathrm{C}$ one-bond correlation map from HSQC NMR experiment of compound 3 in $\mathrm{CDCl}_{3}$ at 500 and $125 \mathrm{MHz}$.

Figure S46. ${ }^{1} \mathrm{H}-{ }^{13} \mathrm{C}$ long-range correlation map from HMBC NMR experiment of compound 3 in $\mathrm{CDCl}_{3}$ at 500 and $125 \mathrm{MHz}$.

Figure S47. Enlargement $(\delta 7.60-5.93)$ of ${ }^{1} \mathrm{H}-{ }^{13} \mathrm{C}$ long-range correlation map from HMBC NMR experiment of compound 3 in $\mathrm{CDCl}_{3}$ at 500 and $125 \mathrm{MHz}$. 
Figure S48. Enlargement $(\delta 4.72-2.55)$ of ${ }^{1} \mathrm{H}-{ }^{13} \mathrm{C}$ long-range correlation map from HMBC NMR experiment of compound 3 in $\mathrm{CDCl}_{3}$ at 500 and $125 \mathrm{MHz}$.

Figure S49. Enlargement of $\mathrm{NOE}(\delta 7.20)$ experiments of compound 3 in $\mathrm{CDCl}_{3}$ at $500 \mathrm{MHz}$.

Figure S50. Enlargement of NOE ( $\delta$ 6.95) experiments of compound 3 in $\mathrm{CDCl}_{3}$ at $500 \mathrm{MHz}$.

Figure S51. Enlargement of $\mathrm{NOE}(\delta 6.67)$ experiments of compound 3 in $\mathrm{CDCl}_{3}$ at $500 \mathrm{MHz}$.

Figure S52. Enlargement of $\mathrm{NOE}(\delta 4.43)$ experiments of compound 3 in $\mathrm{CDCl}_{3}$ at $500 \mathrm{MHz}$.

Figure S53. Enlargement of $\mathrm{NOE}(\delta 4.22)$ experiments of compound 3 in $\mathrm{CDCl}_{3}$ at $500 \mathrm{MHz}$.

Figure S54. Enlargement of $\mathrm{NOE}(\delta 3.22)$ experiments of compound 3 in $\mathrm{CDCl}_{3}$ at $500 \mathrm{MHz}$.

Figure S55. Enlargement of $\mathrm{NOE}(\delta 3.00)$ experiments of compound 3 in $\mathrm{CDCl}_{3}$ at $500 \mathrm{MHz}$.

Figure S56. Enlargement of $\mathrm{NOE}(\delta 2.55)$ experiments of compound 3 in $\mathrm{CDCl}_{3}$ at $500 \mathrm{MHz}$.

Figure S57. HRESIMS spectrum of compound 3.

Figure S58. Extracted ion chromatogram, MS and MS/MS spectra of compound 3.

Figure S59. ${ }^{1} \mathrm{H}$ NMR spectrum of compound 4 in $\mathrm{CDCl}_{3}$ at $500 \mathrm{MHz}$.

Figure S60. Enlargement ( $\delta$ 8.64-6.09) of ${ }^{1} \mathrm{H}$ NMR spectrum of compound 4 in $\mathrm{CDCl}_{3}$ at 500 $\mathrm{MHz}$.

Figure S61. Enlargement ( $\delta$ 8.64-7.93) of ${ }^{1} \mathrm{H}$ NMR spectrum of compound 4 in $\mathrm{CDCl}_{3}$ at 500 $\mathrm{MHz}$.

Figure S62. Enlargement ( $\delta$ 7.26-6.09) of ${ }^{1} \mathrm{H}$ NMR spectrum of compound 4 in $\mathrm{CDCl}_{3}$ at 500 $\mathrm{MHz}$.

Figure S63. Enlargement ( $\delta$ 3.94-3.10) of ${ }^{1} \mathrm{H}$ NMR spectrum of compound 4 in $\mathrm{CDCl}_{3}$ at 500 $\mathrm{MHz}$.

Figure S64. Enlargement ( $\delta 3.69-3.10)$ of ${ }^{1} \mathrm{H}$ NMR spectrum of compound 4 in $\mathrm{CDCl}_{3}$ at 500 $\mathrm{MHz}$.

Figure S65. ${ }^{13} \mathrm{C}$ NMR spectrum of compound 4 in $\mathrm{CDCl}_{3}$ at $125 \mathrm{MHz}$.

Figure S66. Enlargement ( $\delta$ 183.5-100.6) of ${ }^{13} \mathrm{C}$ NMR spectrum of compound 4 in $\mathrm{CDCl}_{3}$ at $125 \mathrm{MHz}$.

Figure S67. Enlargement ( $\delta 55.7-36.0)$ of ${ }^{13} \mathrm{C}$ NMR spectrum of compound 4 in $\mathrm{CDCl}_{3}$ at 125 $\mathrm{MHz}$.

Figure S68. ${ }^{1} \mathrm{H}-{ }^{1} \mathrm{H}$ COSY correlation map from ${ }^{1} \mathrm{H}$ NMR experiment of compound 4 in $\mathrm{CDCl}_{3}$ at $500 \mathrm{MHz}$.

Figure S69. Enlargement $\left(\delta\right.$ 8.64-7.21) of ${ }^{1} \mathrm{H}^{1}{ }^{1} \mathrm{H}$ COSY correlation map from ${ }^{1} \mathrm{H}$ NMR experiment of compound 4 in $\mathrm{CDCl}_{3}$ at $500 \mathrm{MHz}$.

Figure S70. Enlargement $\left(\delta\right.$ 3.68-3.11) of ${ }^{1} \mathrm{H}^{-1} \mathrm{H}$ COSY correlation map from ${ }^{1} \mathrm{H}$ NMR experiment of compound 4 in $\mathrm{CDCl}_{3}$ at $500 \mathrm{MHz}$. 
Figure S71. ${ }^{1} \mathrm{H}-{ }^{13} \mathrm{C}$ one-bond correlation map from HSQC NMR experiment of compound 4 in $\mathrm{CDCl}_{3}$ at 500 and $125 \mathrm{MHz}$.

Figure S72. Enlargement $(\delta 8.64-6.08)$ of ${ }^{1} \mathrm{H}-{ }^{13} \mathrm{C}$ one-bond correlation map from HSQC NMR experiment of compound 4 in $\mathrm{CDCl}_{3}$ at 500 and $125 \mathrm{MHz}$.

Figure S73. Enlargement $(\delta 4.05-3.11)$ of ${ }^{1} \mathrm{H}-{ }^{13} \mathrm{C}$ one-bond correlation map from HSQC NMR experiment of compound 4 in $\mathrm{CDCl}_{3}$ at 500 and $125 \mathrm{MHz}$.

Figure S74. ${ }^{1} \mathrm{H}-{ }^{13} \mathrm{C}$ long-range correlation map from HMBC NMR experiment of compound 4 in $\mathrm{CDCl}_{3}$ at 500 and $125 \mathrm{MHz}$.

Figure S75. Enlargement $\left(\delta\right.$ 8.64-6.08) of ${ }^{1} \mathrm{H}-{ }^{13} \mathrm{C}$ long-range correlation map from HMBC NMR experiment of compound 4 in $\mathrm{CDCl}_{3}$ at 500 and $125 \mathrm{MHz}$.

Figure S76. Enlargement ( $\delta$ 3.94-3.11) of ${ }^{1} \mathrm{H}-{ }^{13} \mathrm{C}$ long-range correlation map from HMBC NMR experiment of compound 4 in $\mathrm{CDCl}_{3}$ at 500 and $125 \mathrm{MHz}$.

Figure S77. HRESIMS spectrum of compound 4.

Figure S78. ESIMS/MS spectrum of compound 4.

Figure S79. ${ }^{1} \mathrm{H}$ NMR spectrum of compound 5 in $\mathrm{CDCl}_{3}$ at $500 \mathrm{MHz}$.

Figure S80. Enlargement ( $\delta$ 8.23-6.09) of ${ }^{1} \mathrm{H}$ NMR spectrum of compound $\mathbf{5}$ in $\mathrm{CDCl}_{3}$ at 500 $\mathrm{MHz}$.

Figure S81. Enlargement ( $\delta$ 4.02-3.08) of ${ }^{1} \mathrm{H}$ NMR spectrum of compound 5 in $\mathrm{CDCl}_{3}$ at 500 $\mathrm{MHz}$.

Figure S82. ${ }^{13} \mathrm{C}$ NMR spectrum of compound 5 in $\mathrm{CDCl}_{3}$ at $125 \mathrm{MHz}$.

Figure S83. Enlargement ( $\delta$ 182.9-125.8) of ${ }^{13} \mathrm{C}$ NMR spectrum of compound $\mathbf{5}$ in $\mathrm{CDCl}_{3}$ at $125 \mathrm{MHz}$.

Figure S84. Enlargement $(\delta 111.6-36.0)$ of ${ }^{13} \mathrm{C}$ NMR spectrum of compound 5 in $\mathrm{CDCl}_{3}$ at 125 $\mathrm{MHz}$.

Figure S85. ${ }^{13} \mathrm{C}$ NMR DEP135 spectrum of compound 5 in $\mathrm{CDCl}_{3}$ at $125 \mathrm{MHz}$.

Figure S86. Enlargement ( $\delta$ 152.0-100.6) of ${ }^{13} \mathrm{C}$ NMR DEP135 spectrum of compound 5 in $\mathrm{CDCl}_{3}$ at $125 \mathrm{MHz}$.

Figure S87. Enlargement ( $\delta 56.0-36.0)$ of ${ }^{13} \mathrm{C}$ NMR DEP135 spectrum of compound 3 in $\mathrm{CDCl}_{3}$ at $125 \mathrm{MHz}$.

Figure S88. ${ }^{1} \mathrm{H}-{ }^{1} \mathrm{H}$ COSY correlation map from ${ }^{1} \mathrm{H}$ NMR experiment of compound 5 in $\mathrm{CDCl}_{3}$ at $500 \mathrm{MHz}$.

Figure S89. Enlargement $\left(\delta\right.$ 8.23-6.09) of ${ }^{1} \mathrm{H}-{ }^{1} \mathrm{H}$ COSY correlation map from ${ }^{1} \mathrm{H}$ NMR experiment of compound 5 in $\mathrm{CDCl}_{3}$ at $500 \mathrm{MHz}$.

Figure S90. Enlargement $\left(\delta\right.$ 4.02-3.08) of ${ }^{1} \mathrm{H}-{ }^{1} \mathrm{H}$ COSY correlation map from ${ }^{1} \mathrm{H}$ NMR experiment of compound 5 in $\mathrm{CDCl}_{3}$ at $500 \mathrm{MHz}$. 
Figure S91. ${ }^{1} \mathrm{H}-{ }^{13} \mathrm{C}$ one-bond correlation map from HSQC NMR experiment of compound 5 in $\mathrm{CDCl}_{3}$ at 500 and $125 \mathrm{MHz}$.

Figure S92. Enlargement $(\delta 8.23-6.09)$ of ${ }^{1} \mathrm{H}-{ }^{13} \mathrm{C}$ one-bond correlation map from HSQC NMR experiment of compound 5 in $\mathrm{CDCl}_{3}$ at 500 and $125 \mathrm{MHz}$.

Figure S93. Enlargement $(\delta 4.02-3.08)$ of ${ }^{1} \mathrm{H}-{ }^{13} \mathrm{C}$ one-bond correlation map from HSQC NMR experiment of compound 5 in $\mathrm{CDCl}_{3}$ at 500 and $125 \mathrm{MHz}$.

Figure S94. ${ }^{1} \mathrm{H}-{ }^{13} \mathrm{C}$ long-range correlation map from HMBC NMR experiment of compound 5 in $\mathrm{CDCl}_{3}$ at 500 and $125 \mathrm{MHz}$.

Figure S95. Enlargement $(\delta 8.23-6.09)$ of ${ }^{1} \mathrm{H}-{ }^{13} \mathrm{C}$ long-range correlation map from HMBC NMR experiment of compound 5 in $\mathrm{CDCl}_{3}$ at 500 and $125 \mathrm{MHz}$.

Figure S96. Enlargement $\left(\delta\right.$ 4.02-3.08) of ${ }^{1} \mathrm{H}-{ }^{13} \mathrm{C}$ long-range correlation map from HMBC NMR experiment of compound $\mathbf{5}$ in $\mathrm{CDCl}_{3}$ at 500 and $125 \mathrm{MHz}$.

Figure S97. HRESIMS spectrum of compound 5.

Figure S98. Extracted ion chromatogram, MS and MS/MS spectra of compound 5.

Figure S99. ${ }^{1} \mathrm{H}$ NMR spectrum of compound 10 in $\mathrm{CDCl}_{3}$ at $500 \mathrm{MHz}$.

Figure S100. Enlargement $\left(\delta\right.$ 7.26-6.52) of ${ }^{1} \mathrm{H}$ NMR spectrum of compound 10 in $\mathrm{CDCl}_{3}$ at $500 \mathrm{MHz}$.

Figure S101. Enlargement $(\delta 5.01-3.63)$ of ${ }^{1} \mathrm{H}$ NMR spectrum of compound 10 in $\mathrm{CDCl}_{3}$ at $500 \mathrm{MHz}$.

Figure S102. ${ }^{13} \mathrm{C}$ NMR spectrum of compound 10 in $\mathrm{CDCl}_{3}$ at $125 \mathrm{MHz}$.

Figure S103. Enlargement ( $\delta$ 150.7-97.3) of ${ }^{13} \mathrm{C}$ NMR spectrum of compound 10 in $\mathrm{CDCl}_{3}$ at $125 \mathrm{MHz}$.

Figure S104. Enlargement $\left(\delta\right.$ 70.8-56.1) of ${ }^{13} \mathrm{C}$ NMR spectrum of compound 10 in $\mathrm{CDCl}_{3}$ at $125 \mathrm{MHz}$.

Figure S105. ${ }^{13} \mathrm{C}$ NMR DEP135 spectrum of compound 10 in $\mathrm{CDCl}_{3}$ at $125 \mathrm{MHz}$.

Figure S106. Enlargement ( $\delta$ 111.2-56.1) of ${ }^{13} \mathrm{C}$ NMR DEP135 spectrum of compound 10 in $\mathrm{CDCl}_{3}$ at $125 \mathrm{MHz}$.

Figure S107. Enlargement ( $\delta 70.8-56.1)$ of ${ }^{13} \mathrm{C}$ NMR DEP135 spectrum of compound 10 in $\mathrm{CDCl}_{3}$ at $125 \mathrm{MHz}$.

Figure S108. ${ }^{1} \mathrm{H}-{ }^{1} \mathrm{H}$ COSY correlation map from ${ }^{1} \mathrm{H}$ NMR experiment of compound 10 in $\mathrm{CDCl}_{3}$ at $500 \mathrm{MHz}$.

Figure S109. Enlargement $\left(\delta\right.$ 7.26-3.63) of ${ }^{1} \mathrm{H}-{ }^{1} \mathrm{H}$ COSY correlation map from ${ }^{1} \mathrm{H}$ NMR experiment of compound 10 in $\mathrm{CDCl}_{3}$ at $500 \mathrm{MHz}$.

Figure S110. Enlargement $\left(\delta\right.$ 5.01-3.63) of ${ }^{1} \mathrm{H}-{ }^{1} \mathrm{H}$ COSY correlation map from ${ }^{1} \mathrm{H}$ NMR experiment of compound $\mathbf{1 0}$ in $\mathrm{CDCl}_{3}$ at $500 \mathrm{MHz}$. 
Figure S111. ${ }^{1} \mathrm{H}-{ }^{13} \mathrm{C}$ one-bond correlation map from HSQC NMR experiment of compound $\mathbf{1 0}$ in $\mathrm{CDCl}_{3}$ at 500 and $125 \mathrm{MHz}$.

Figure S112. Enlargement $(\delta 8.23-6.09)$ of ${ }^{1} \mathrm{H}-{ }^{13} \mathrm{C}$ one-bond correlation map from HSQC NMR experiment of compound $\mathbf{1 0}$ in $\mathrm{CDCl}_{3}$ at 500 and $125 \mathrm{MHz}$.

Figure S113. Enlargement $(\delta 4.02-3.08)$ of ${ }^{1} \mathrm{H}-{ }^{13} \mathrm{C}$ one-bond correlation map from HSQC NMR experiment of compound 10 in $\mathrm{CDCl}_{3}$ at 500 and $125 \mathrm{MHz}$.

Figure S114. ${ }^{1} \mathrm{H}-{ }^{13} \mathrm{C}$ long-range correlation map from HMBC NMR experiment of compound 10 in $\mathrm{CDCl}_{3}$ at 500 and $125 \mathrm{MHz}$.

Figure S115. Enlargement $(\delta 7.45-6.50)$ of ${ }^{1} \mathrm{H}-{ }^{13} \mathrm{C}$ long-range correlation map from HMBC NMR experiment of compound $\mathbf{1 0}$ in $\mathrm{CDCl}_{3}$ at 500 and $125 \mathrm{MHz}$.

Figure S116. Enlargement $(\delta 5.00-3.60)$ of ${ }^{1} \mathrm{H}-{ }^{13} \mathrm{C}$ long-range correlation map from HMBC NMR experiment of compound 10 in $\mathrm{CDCl}_{3}$ at 500 and $125 \mathrm{MHz}$.

Figure S117. HRESIMS spectrum of compound 10.

Figure S118. Structures of alkaloids 2, 6, 7, 9 and 11 whose NMR data were fully assigned.

Figure S119. ${ }^{1} \mathrm{H}$ NMR spectrum of compound 1 in $\mathrm{CDCl}_{3}$ at $500 \mathrm{MHz}$.

Figure S120. Enlargement ( $\delta 7.69-6.88)$ of ${ }^{1} \mathrm{H}$ NMR spectrum of compound 1 in $\mathrm{CDCl}_{3}$ at 500 $\mathrm{MHz}$.

Figure S121. Enlargement ( $83.94-3.89)$ of ${ }^{1} \mathrm{H}$ NMR spectrum of compound $\mathbf{1}$ in $\mathrm{CDCl}_{3}$ at 500 $\mathrm{MHz}$.

Figure S122. ${ }^{1} \mathrm{H}$ NMR spectrum of compound 2 in $\mathrm{CDCl}_{3}$ at $500 \mathrm{MHz}$.

Figure S123. Enlargement ( $\delta 7.66-6.52)$ of ${ }^{1} \mathrm{H}$ NMR spectrum of compound 2 in $\mathrm{CDCl}_{3}$ at 500 $\mathrm{MHz}$.

Figure S124. Enlargement ( $\delta 6.08-5.93)$ of ${ }^{1} \mathrm{H}$ NMR spectrum of compound 2 in $\mathrm{CDCl}_{3}$ at 500 $\mathrm{MHz}$.

Figure S125. Enlargement ( $\delta 3.92-2.57)$ of ${ }^{1} \mathrm{H}$ NMR spectrum of compound 2 in $\mathrm{CDCl}_{3}$ at 500 $\mathrm{MHz}$.

Figure S126. ${ }^{1} \mathrm{H}$ NMR spectrum of compound 6 in $\mathrm{CDCl}_{3}$ at $500 \mathrm{MHz}$.

Figure S127. Enlargement ( $\delta 7.58-6.52)$ of ${ }^{1} \mathrm{H}$ NMR spectrum of compound 6 in $\mathrm{CDCl}_{3}$ at 500 $\mathrm{MHz}$.

Figure S128. Enlargement $\left(\delta\right.$ 6.11-4.93) of ${ }^{1} \mathrm{H}$ NMR spectrum of compound 6 in $\mathrm{CDCl}_{3}$ at 500 MHz.

Figure S129. Enlargement ( $\delta$ 4.07-2.76) of ${ }^{1} \mathrm{H}$ NMR spectrum of compound 6 in $\mathrm{CDCl}_{3}$ at 500 $\mathrm{MHz}$.

Figure S130. ${ }^{1} \mathrm{H}$ NMR spectrum of compound 7 in $\mathrm{CDCl}_{3}$ at $500 \mathrm{MHz}$. 
Figure S131. Enlargement $(\delta 7.63-7.26)$ of ${ }^{1} \mathrm{H}$ NMR spectrum of compound 7 in $\mathrm{CDCl}_{3}$ at 500 $\mathrm{MHz}$.

Figure S132. Enlargement $(\delta 6.53-5.95)$ of ${ }^{1} \mathrm{H}$ NMR spectrum of compound 7 in $\mathrm{CDCl}_{3}$ at 500 $\mathrm{MHz}$.

Figure S133. Enlargement ( $\delta 4.61-3.50)$ of ${ }^{1} \mathrm{H}$ NMR spectrum of compound 7 in $\mathrm{CDCl}_{3}$ at 500 $\mathrm{MHz}$.

Figure S134. Enlargement ( $\delta 3.08-2.59)$ of ${ }^{1} \mathrm{H}$ NMR spectrum of compound 7 in $\mathrm{CDCl}_{3}$ at 500 $\mathrm{MHz}$.

Figure S135. ${ }^{1} \mathrm{H}$ NMR spectrum of compound 8 in $\mathrm{CDCl}_{3}$ at $500 \mathrm{MHz}$.

Figure S136. Enlargement $(\delta 6.78-6.33)$ of ${ }^{1} \mathrm{H}$ NMR spectrum of compound 8 in $\mathrm{CDCl}_{3}$ at 500 $\mathrm{MHz}$.

Figure S137. Enlargement ( $\delta 3.90-2.98)$ of ${ }^{1} \mathrm{H}$ NMR spectrum of compound 8 in $\mathrm{CDCl}_{3}$ at 500 $\mathrm{MHz}$.

Figure S138. Enlargement ( $\delta 2.59-1.81)$ of ${ }^{1} \mathrm{H}$ NMR spectrum of compound 8 in $\mathrm{CDCl}_{3}$ at 500 $\mathrm{MHz}$.

Figure S139. ${ }^{1} \mathrm{H}$ NMR spectrum of compound 9 in $\mathrm{CDCl}_{3}+$ drops of $\mathrm{CD}_{3} \mathrm{OD}$ at $300 \mathrm{MHz}$.

Figure S140. Enlargement ( $\delta 8.80-6.38)$ of ${ }^{1} \mathrm{H}$ NMR spectrum of compound 9 in $\mathrm{CDCl}_{3}+$ drops of $\mathrm{CD}_{3} \mathrm{OD}$ at $300 \mathrm{MHz}$.

Figure S141. Enlargement ( $\delta 4.06-4.00)$ of ${ }^{1} \mathrm{H}$ NMR spectrum of compound 9 in $\mathrm{CDCl}_{3}+$ drops of $\mathrm{CD}_{3} \mathrm{OD}$ at $300 \mathrm{MHz}$.

Figure S142. ${ }^{1} \mathrm{H}$ NMR spectrum of compound 11 in $\mathrm{CDCl}_{3}$ at $500 \mathrm{MHz}$.

Figure S143. Enlargement $\left(\delta\right.$ 7.62-6.55) of ${ }^{1} \mathrm{H}$ NMR spectrum of compound 11 in $\mathrm{CDCl}_{3}$ at $500 \mathrm{MHz}$.

Figure S144. Enlargement ( $\delta$ 6.17-6.00) of ${ }^{1} \mathrm{H}$ NMR spectrum of compound 11 in $\mathrm{CDCl}_{3}$ at $500 \mathrm{MHz}$.

Figure S145. Enlargement ( $\delta 5.19-3.92)$ of ${ }^{1} \mathrm{H}$ NMR spectrum of compound 11 in $\mathrm{CDCl}_{3}$ at $500 \mathrm{MHz}$.

Figure S146. Enlargement ( $\delta 3.73-2.99)$ of ${ }^{1} \mathrm{H}$ NMR spectrum of compound 11 in $\mathrm{CDCl}_{3}$ at $500 \mathrm{MHz}$. 
Figure S1. Total ion chromatogram of the alkaloid-rich fraction from D. surinamensis.

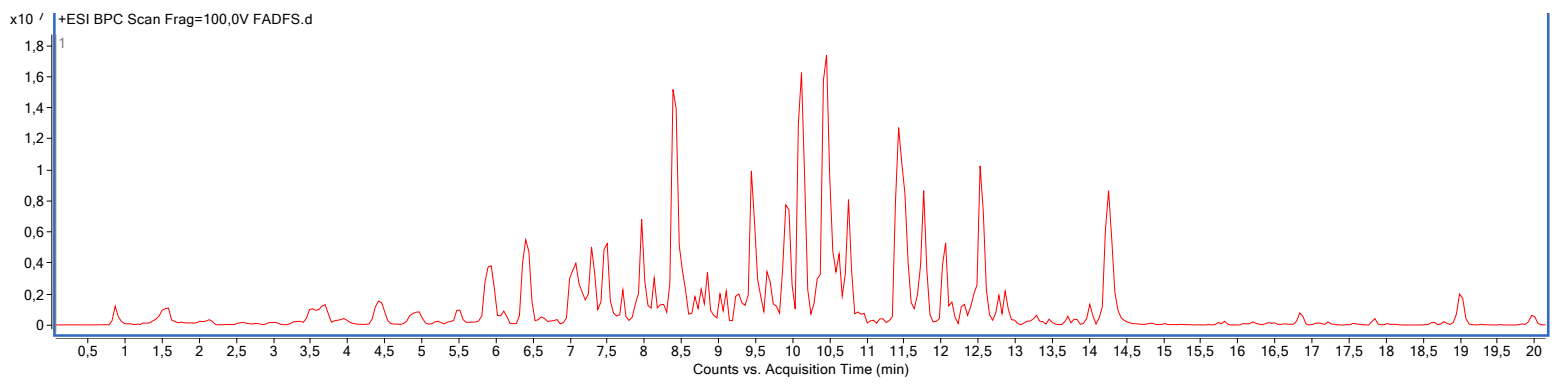


Figure S2. Chemical structures of the dereplicated compounds $2,11,12 a-12 h, 13 a, 13 b, 14 a-$ 14n and 15.<smiles>[R]c1ccc(C[C@@H]2c3cc([R])c([R])cc3CCN2[R6])cc1[R]</smiles><smiles>[R2]c1ccc(C[C@@H]2c3cc(O)c(OC)cc3CC[N+]2(C)[O-])cc1[R]</smiles>

$\begin{array}{llllll} & \mathbf{R}_{\mathbf{1}} & \mathbf{R}_{\mathbf{2}} & \mathbf{R}_{\mathbf{3}} & \mathbf{R}_{\mathbf{4}} & \mathbf{R}_{\mathbf{5}} \\ \mathbf{1 2 a} & \mathrm{OCH}_{3} & \mathrm{OH} & \mathrm{H} & \mathrm{OH} & \mathrm{H} \\ \mathbf{1 2 b} & \mathrm{OCH}_{3} & \mathrm{OH} & \mathrm{H} & \mathrm{OH} & \mathrm{CH}_{3} \\ \mathbf{1 2 c} & \mathrm{OCH}_{3} & \mathrm{OCH} & \mathrm{H} & \mathrm{OCH}_{3} & \mathrm{CH}_{3} \\ \mathbf{1 2 d} & \mathrm{OCH}_{3} & \mathrm{OH} & \mathrm{OH} & \mathrm{OCH}_{3} & \mathrm{CH}_{3} \\ \mathbf{1 2 e} & \mathrm{OCH}_{3} & \mathrm{OH} & \mathrm{H} & \mathrm{OCH}_{3} & \mathrm{CH}_{3} \\ \mathbf{1 2 f} & \mathrm{OCH}_{3} & \mathrm{OCH}_{3} & \mathrm{H} & \mathrm{OCH}_{3} & \mathrm{CHO}\end{array}$

$$
\begin{array}{ccc} 
& \mathbf{R}_{\mathbf{1}} & \mathbf{R}_{\mathbf{2}} \\
\mathbf{1 2 g} & \mathrm{H} & \mathrm{OH} \\
\mathbf{1 2 h} & \mathrm{OH} & \mathrm{OCH}_{3}
\end{array}
$$<smiles>COc1c(OC)c2c3c(nccc3c1OC)C(=O)c1cc(O)ccc1-2</smiles>

$\mathbf{R}$

13b $\mathrm{OCH}_{3}$

\begin{tabular}{|c|c|c|c|c|c|c|c|}
\hline 2 & $\begin{array}{c}\mathbf{R}_{\mathbf{1}} \\
\mathrm{H}\end{array}$ & $\begin{array}{l}\mathbf{R}_{\mathbf{2}} \\
\text {-O-Cl }\end{array}$ & $\begin{array}{r}\mathbf{R}_{\mathbf{3}} \\
\mathrm{H}_{2}-\mathrm{O}-\end{array}$ & $\begin{array}{l}\mathbf{R}_{\mathbf{4}} \\
\mathrm{H}\end{array}$ & $\begin{array}{c}\mathbf{R}_{\mathbf{5}} \\
\mathrm{OCH}_{3}\end{array}$ & $\begin{array}{c}\mathbf{R}_{6} \\
\mathrm{OCH}_{3}\end{array}$ & $\begin{array}{l}\mathbf{R}_{\mathbf{7}} \\
\mathrm{CH}_{3}\end{array}$ \\
\hline $14 a$ & $\mathrm{H}$ & $\mathrm{OH}$ & $\mathrm{OCH}_{3}$ & $\mathrm{H}$ & $\mathrm{H}$ & $\mathrm{H}$ & $\mathrm{H}$ \\
\hline $14 b$ & $\mathrm{H}$ & $\mathrm{OCH}_{3}$ & $\mathrm{OCH}_{3}$ & $\mathrm{H}$ & $\mathrm{OCH}_{3}$ & $\mathrm{OH}$ & $\mathrm{H}$ \\
\hline $14 c$ & $\mathrm{H}$ & $\mathrm{OCH}_{3}$ & $\mathrm{OCH}_{3}$ & $\mathrm{H}$ & $\mathrm{OCH}_{3}$ & $\mathrm{OCH}_{3}$ & $\mathrm{H}$ \\
\hline 14d & $\mathrm{H}$ & $\mathrm{OH}$ & $\mathrm{OCH}_{3}$ & $\mathrm{H}$ & $\mathrm{H}$ & $\mathrm{H}$ & $\mathrm{CH}_{3}$ \\
\hline $14 e$ & $\mathrm{H}$ & $\mathrm{OCH}_{3}$ & $\mathrm{OCH}_{3}$ & $\mathrm{H}$ & $\mathrm{OCH}_{3}$ & $\mathrm{OH}$ & $\mathrm{CH}_{3}$ \\
\hline $14 f$ & $\mathrm{H}$ & $\mathrm{OCH}_{3}$ & $\mathrm{OH}$ & $\mathrm{OH}$ & $\mathrm{OCH}_{3}$ & $\mathrm{H}$ & $\mathrm{CH}_{3}$ \\
\hline $14 \mathrm{~g}$ & $\mathrm{H}$ & $\mathrm{OCH}_{3}$ & $\mathrm{OCH}_{3}$ & $\mathrm{H}$ & $\mathrm{OCH}_{3}$ & $\mathrm{OCH}_{3}$ & $\mathrm{CH}_{3}$ \\
\hline $14 \mathrm{~h}$ & $\mathrm{H}$ & $-\mathrm{O}-\mathrm{C}$ & $\mathrm{H}_{2}-\mathrm{O}-$ & $\mathrm{H}$ & $\mathrm{H}$ & & ${ }_{3} \mathrm{CH}_{3}$ \\
\hline $14 i$ & $\mathrm{H}$ & $\mathrm{OCH}_{3}$ & $\mathrm{OH}$ & $\mathrm{OH}$ & $\mathrm{OCH}_{3}$ & $\mathrm{H}$ & $\mathrm{CHO}$ \\
\hline 14j & $\mathrm{H}$ & $\mathrm{OCH}_{3}$ & $\mathrm{OCH}_{3}$ & $\mathrm{H}$ & $\mathrm{OCH}_{3}$ & $\mathrm{OCH}_{3}$ & $\mathrm{CHO}$ \\
\hline
\end{tabular}<smiles>[R]c1cc2c(c([R])c1[R3])-c1c([R3])c([R])c([R])c3c1[C@@H]([2H])N([R])CC23</smiles>

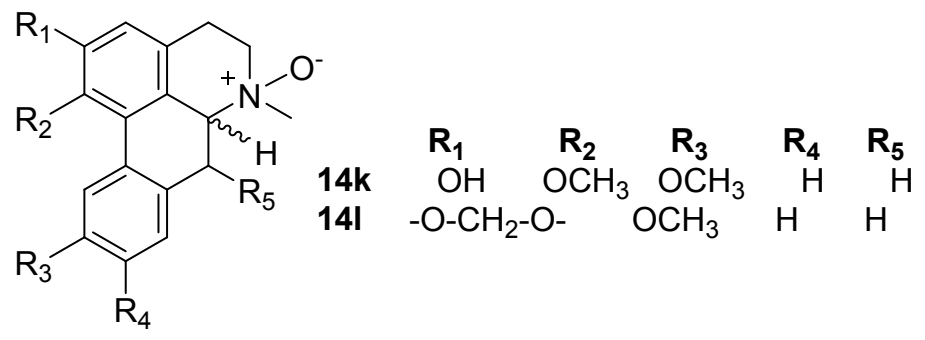<smiles>COc1cc2c3c(c1OC)C1(C=CC(=O)C=C1)CC3NCC2</smiles><smiles>COc1cc2c(cc1OC)CNCC2</smiles> 
Figure S3. Extracted ion chromatogram, MS and MS/MS spectra of compound 12a.
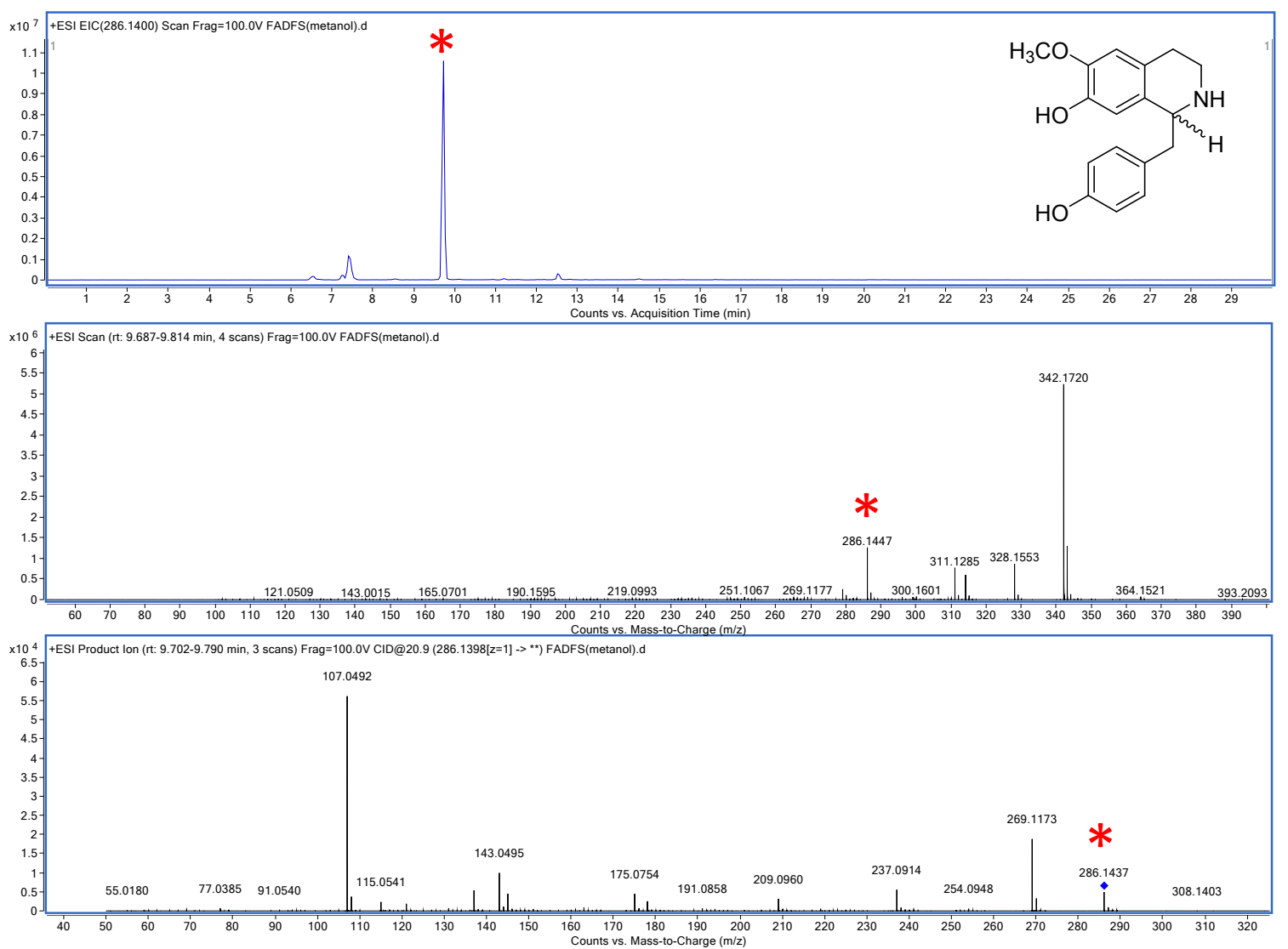

https://gnps.ucsd.edu/ProteoSAFe/gnpslibraryspectrum.jsp?SpectrumID=CCMSLIB0000543 6045\#\%7B\%7D 
Figure S4. Extracted ion chromatogram, MS and MS/MS spectra of compound $\mathbf{1 2 b}$.
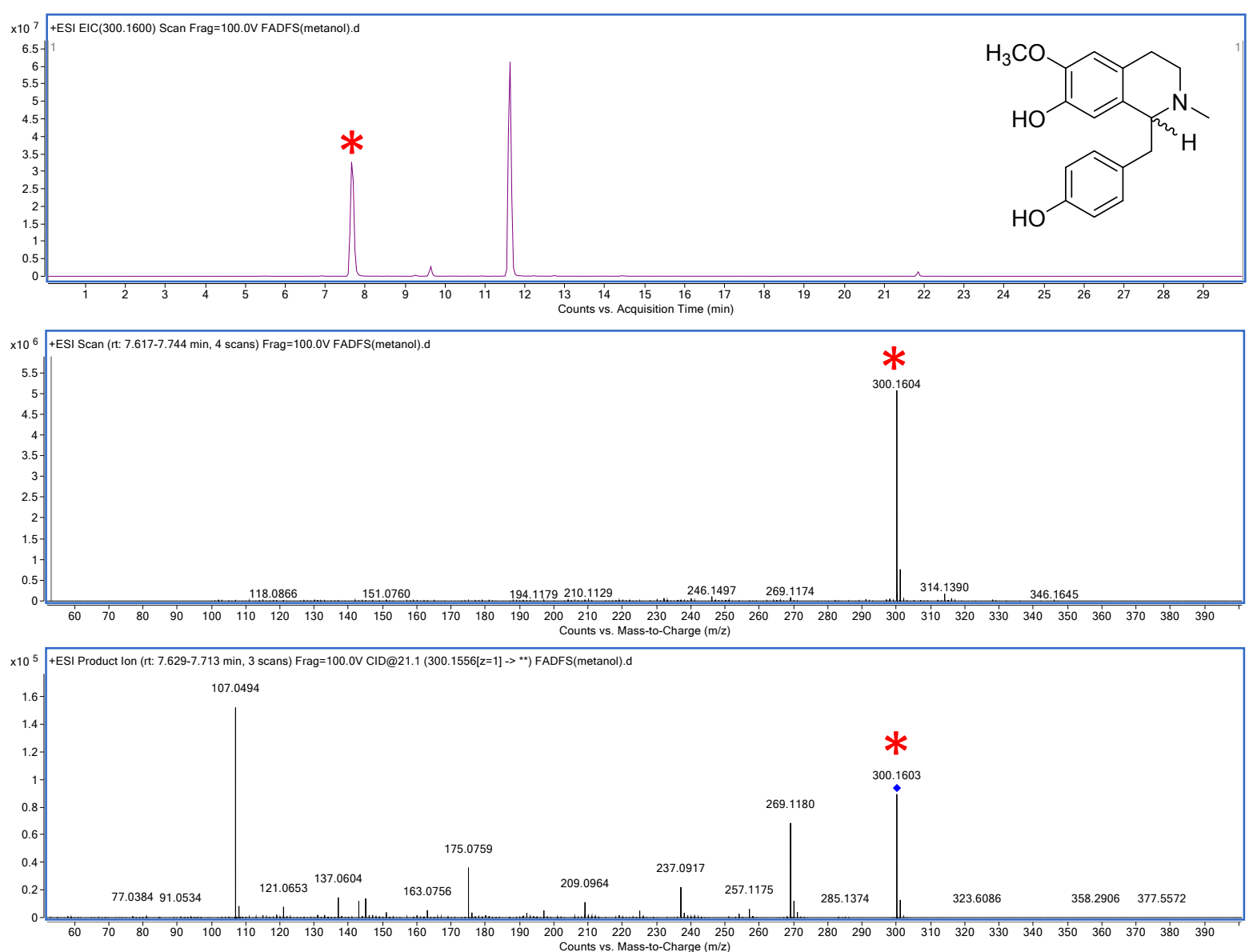

https://gnps.ucsd.edu/ProteoSAFe/gnpslibraryspectrum.jsp?SpectrumID=CCMSLIB0000543 6046\#\%7B\%7D 
Figure S5. Extracted ion chromatogram, MS and MS/MS spectra of compound 12c.
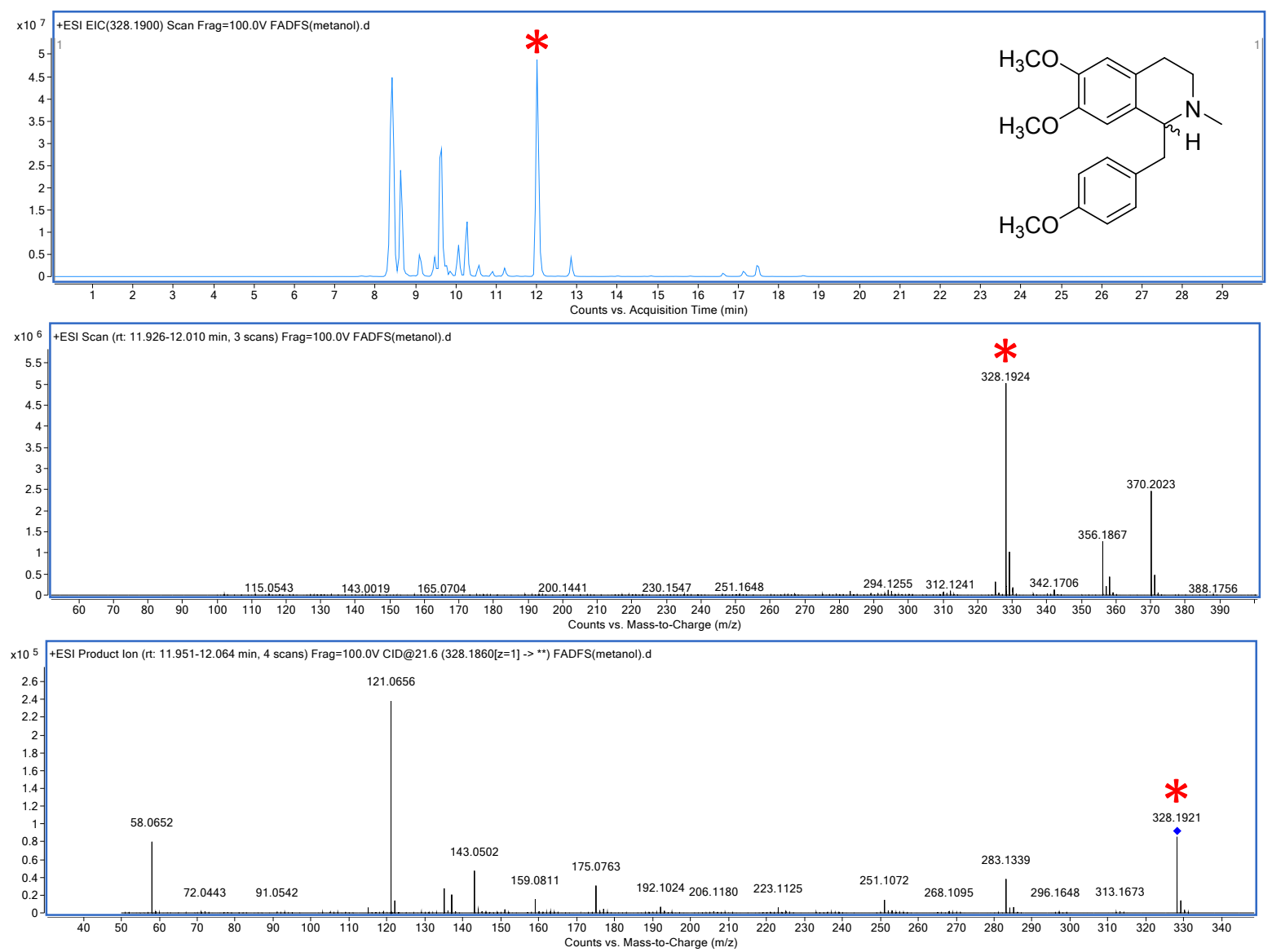

https://gnps.ucsd.edu/ProteoSAFe/gnpslibraryspectrum.jsp?SpectrumID=CCMSLIB0000543 $6047 \# \% 7 \mathrm{~B} \% 7 \mathrm{D}$ 
Figure S6. Extracted ion chromatogram, MS and MS/MS spectra of compound 12d.
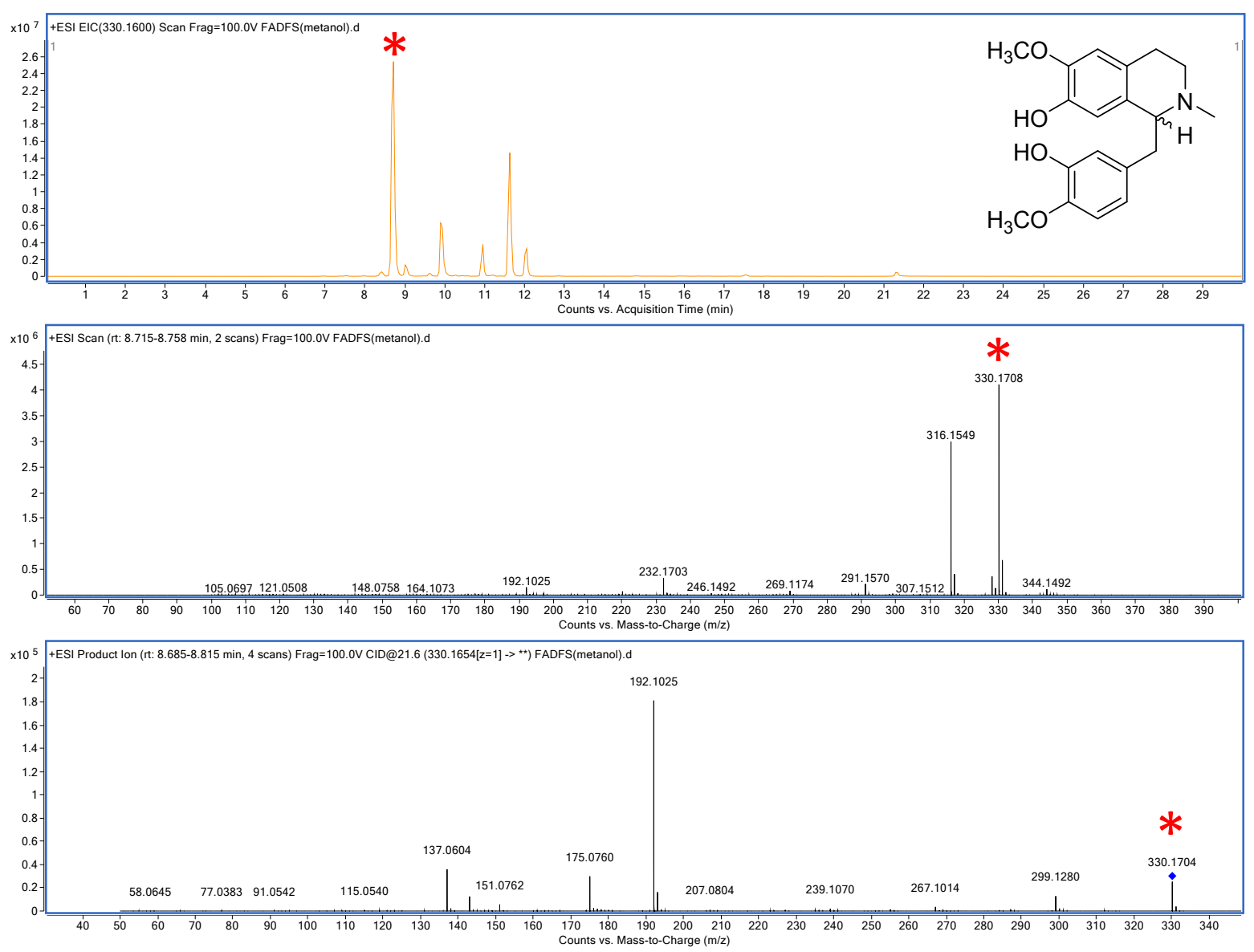

https://gnps.ucsd.edu/ProteoSAFe/gnpslibraryspectrum.jsp?SpectrumID=CCMSLIB0000543 $\underline{6048 \# \% 7 \mathrm{~B} \% 7 \mathrm{D}}$ 
Figure S7. Extracted ion chromatogram, MS and MS/MS spectra of compound 12e.
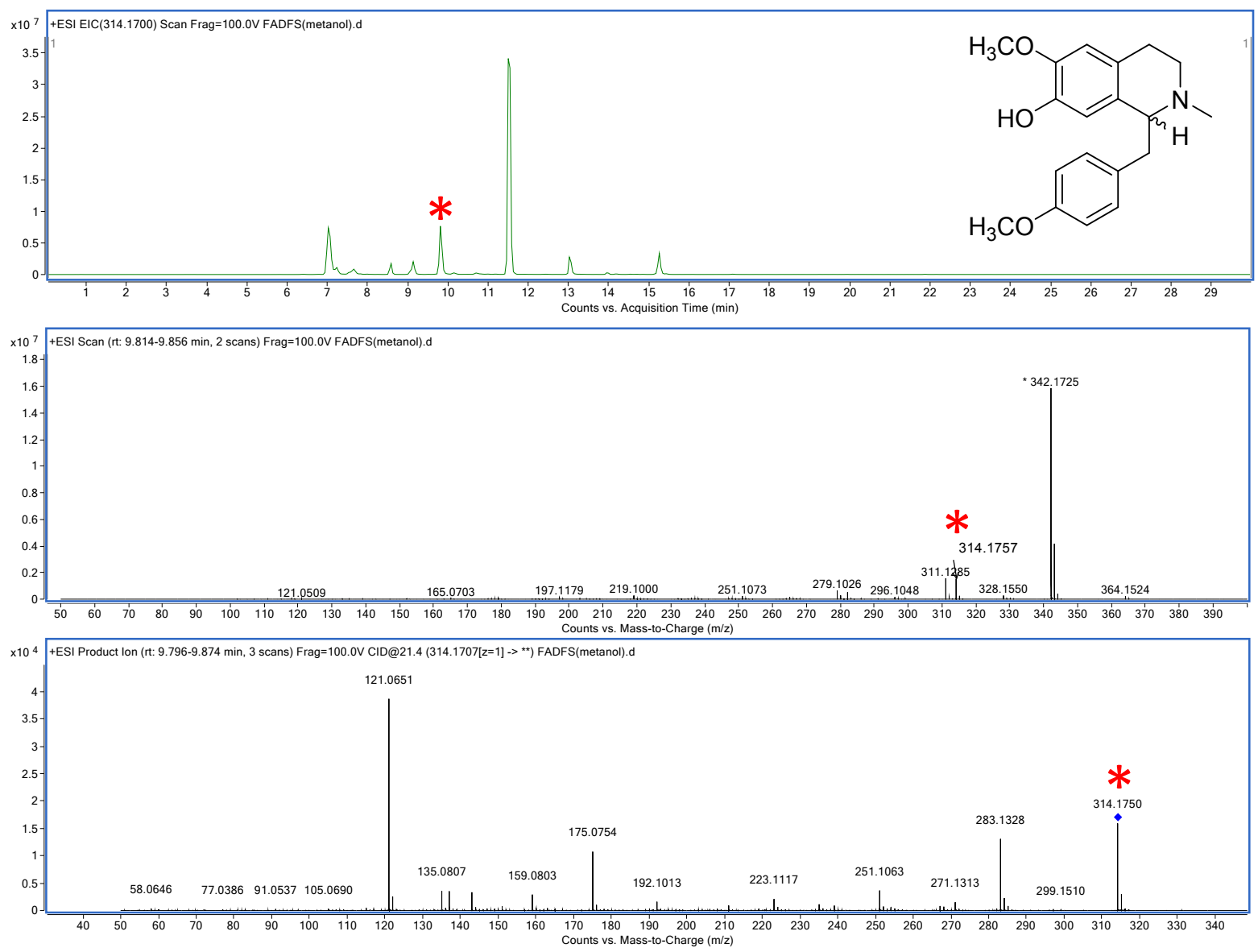

https://gnps.ucsd.edu/ProteoSAFe/gnpslibraryspectrum.jsp?SpectrumID=CCMSLIB0000543 6049\#\%7B\%7D 
Figure S8. Extracted ion chromatogram, MS and MS/MS spectra of compound $\mathbf{1 2 f}$.
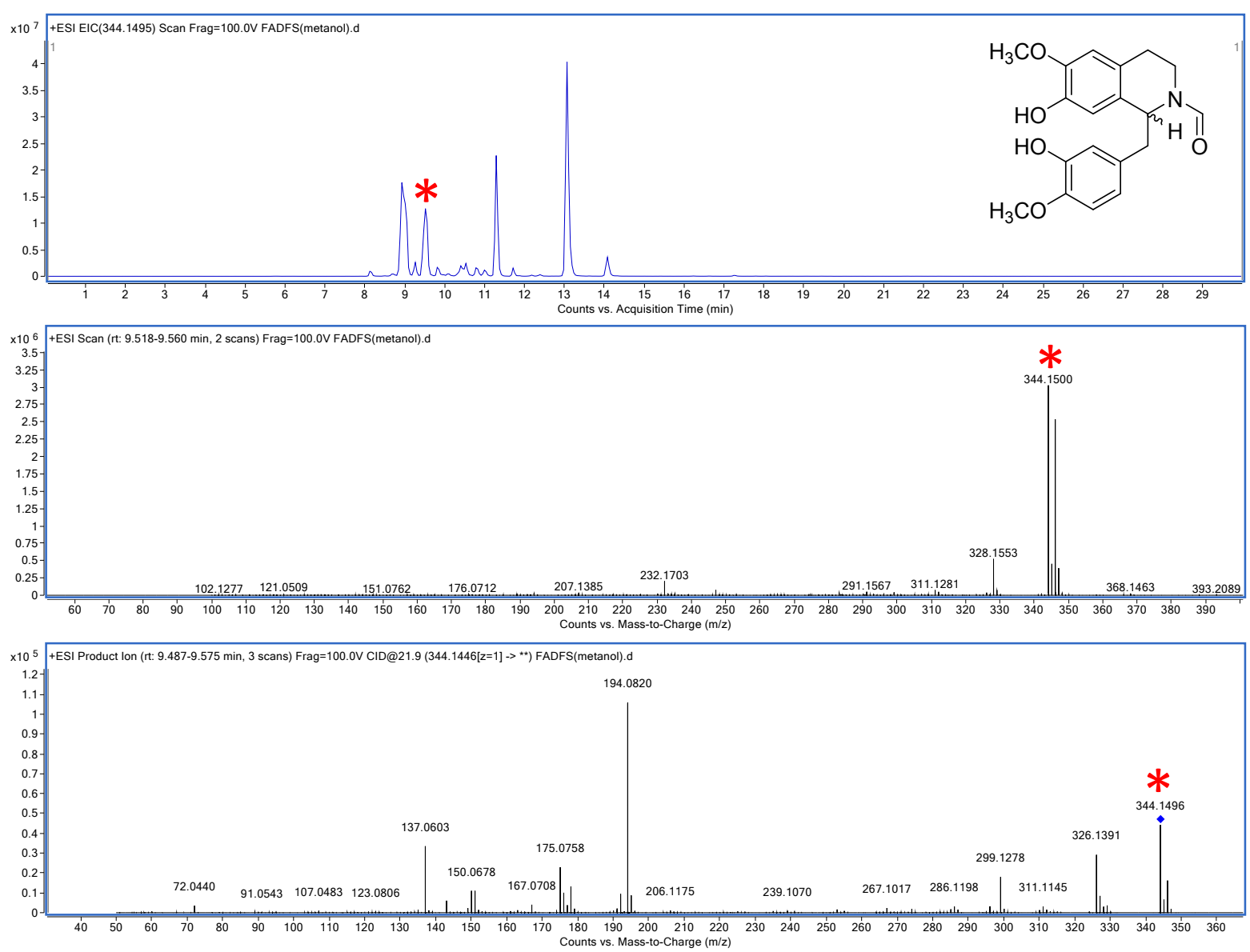

https://gnps.ucsd.edu/ProteoSAFe/gnpslibraryspectrum.jsp?SpectrumID=CCMSLIB0000543 $6050 \# \% 7 \mathrm{~B} \% 7 \mathrm{D}$ 
Figure S9. Extracted ion chromatogram, MS and MS/MS spectra of compound 12g.
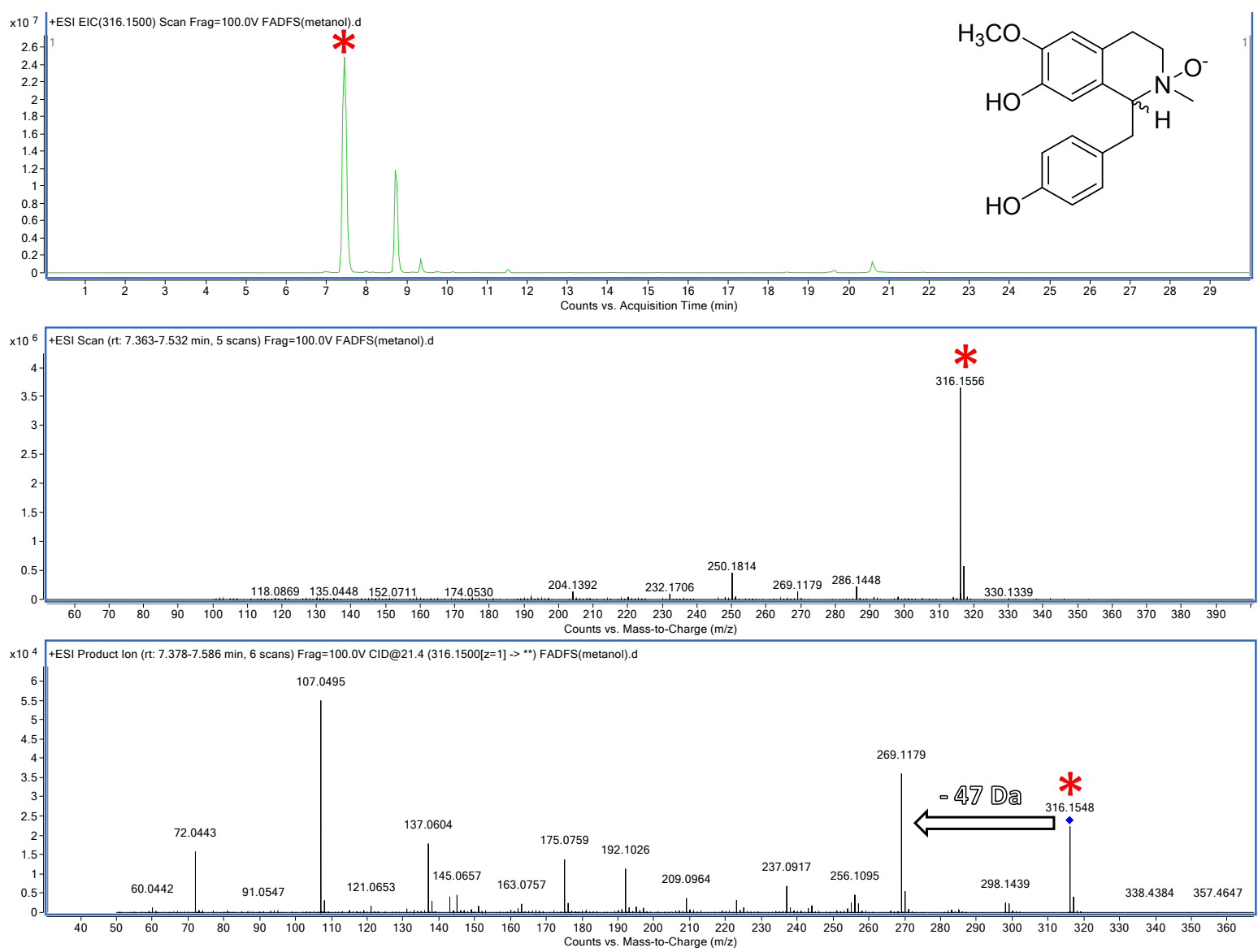

https://gnps.ucsd.edu/ProteoSAFe/gnpslibraryspectrum.jsp?SpectrumID=CCMSLIB0000543 $\underline{6051 \# \% 7 \mathrm{~B} \% 7 \mathrm{D}}$ 
Figure S10. Extracted ion chromatogram, MS and MS/MS spectra of compound $\mathbf{1 2 h}$.
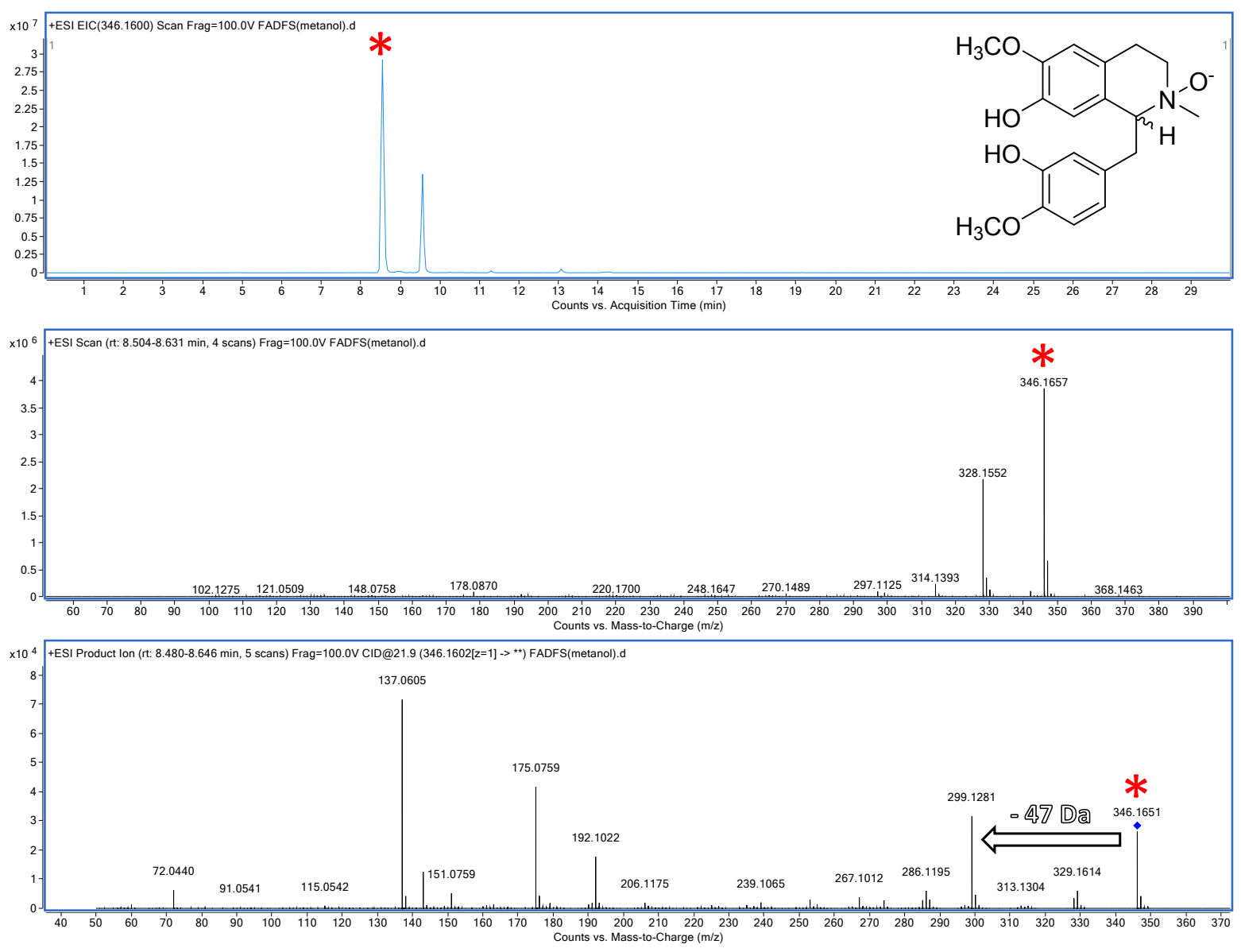

https://gnps.ucsd.edu/ProteoSAFe/gnpslibraryspectrum.jsp?SpectrumID=CCMSLIB0000543 $\underline{6052 \# \% 7 \mathrm{~B} \% 7 \mathrm{D}}$ 
Figure S11. Extracted ion chromatogram, MS and MS/MS spectra of compound 13a.
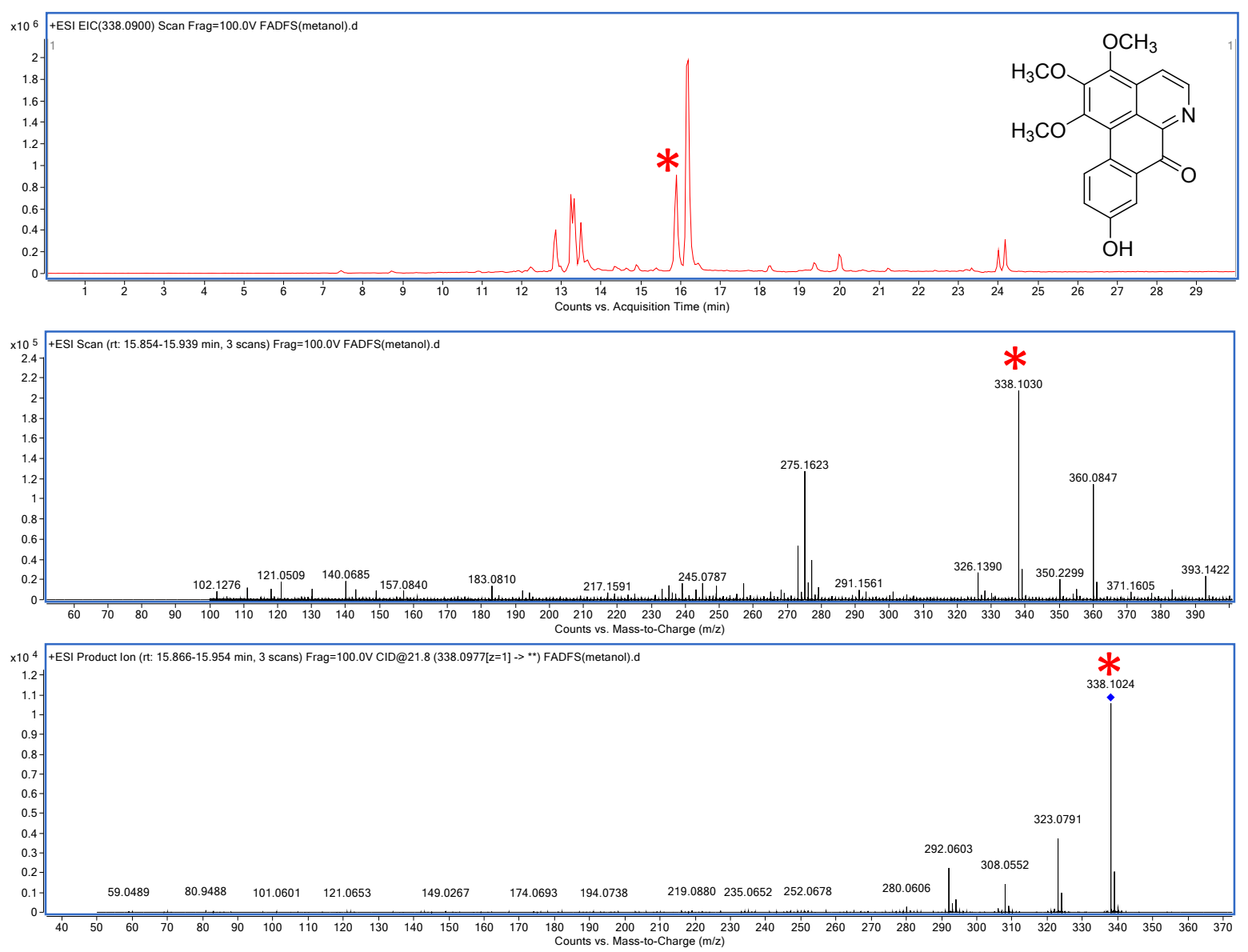

https://gnps.ucsd.edu/ProteoSAFe/gnpslibraryspectrum.jsp?SpectrumID=CCMSLIB0000543 $\underline{6053 \# \% 7 \mathrm{~B} \% 7 \mathrm{D}}$ 
Figure S12. Extracted ion chromatogram, MS and MS/MS spectra of compound 13b.
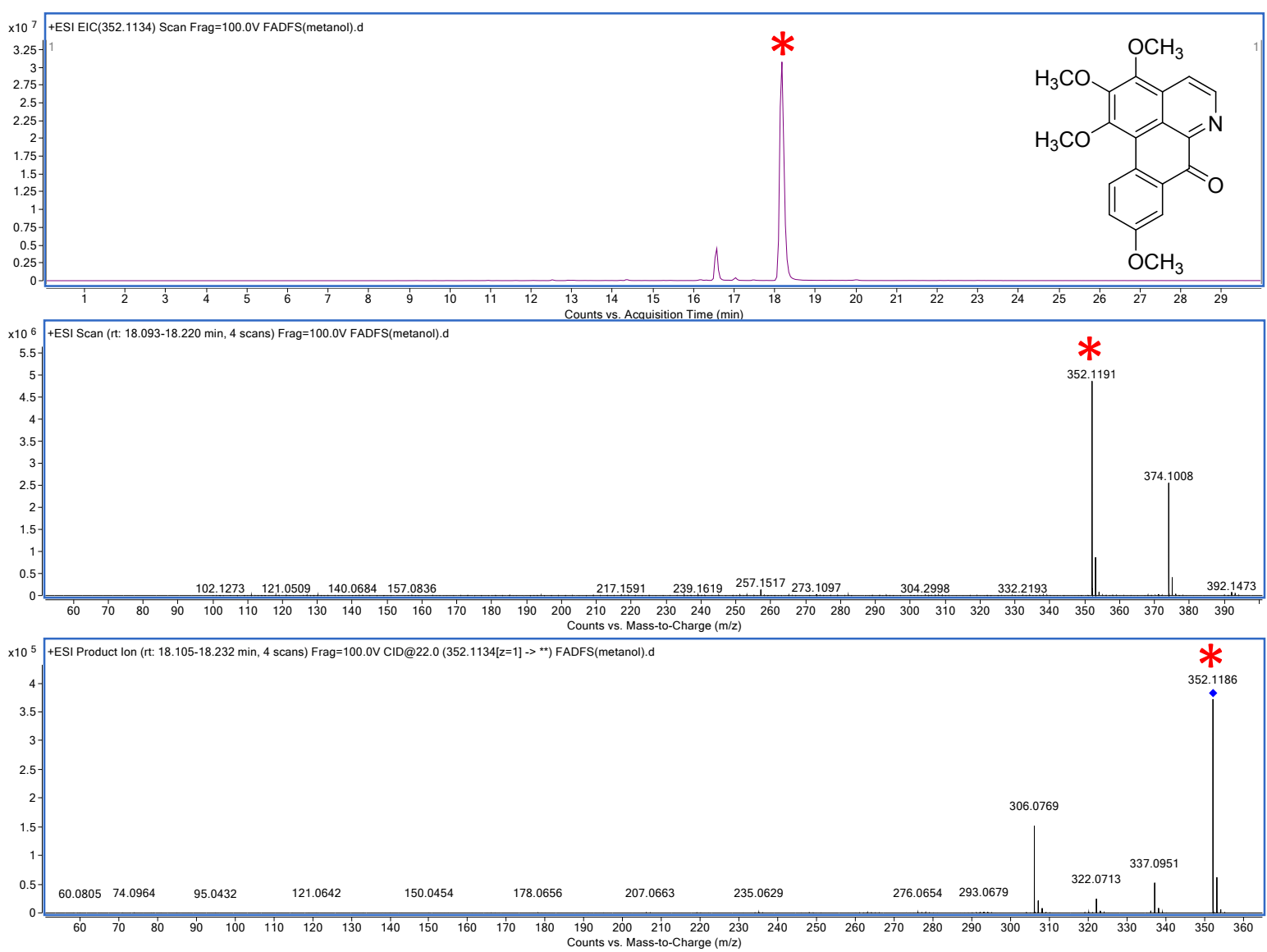

https://gnps.ucsd.edu/ProteoSAFe/gnpslibraryspectrum.jsp?SpectrumID=CCMSLIB0000543 6054\#\%7B\%7D 
Figure S13. Extracted ion chromatogram, MS and MS/MS spectra of compound 2.
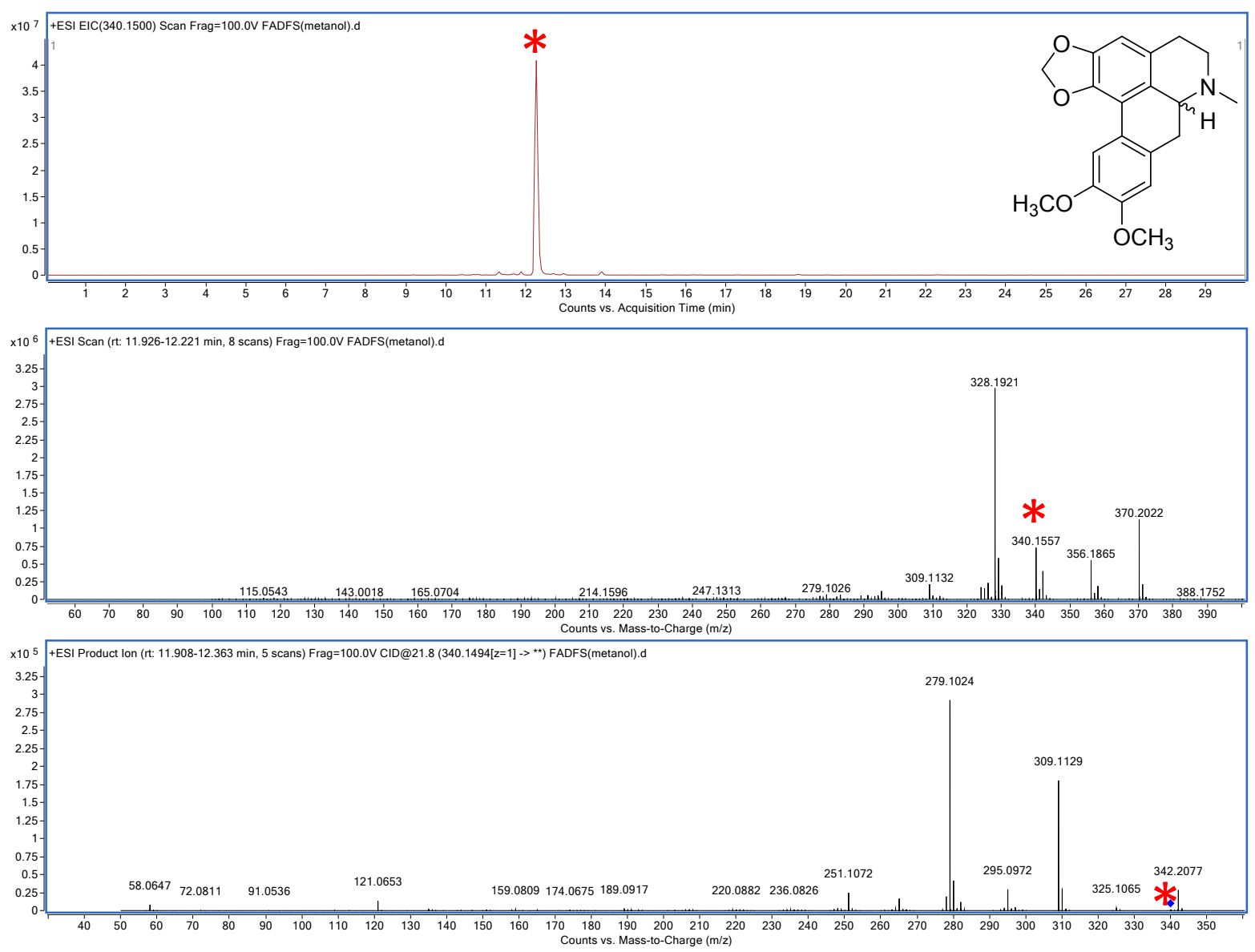

https://gnps.ucsd.edu/ProteoSAFe/gnpslibraryspectrum.jsp?SpectrumID=CCMSLIB0000543 $\underline{6039 \# \% 7 \mathrm{~B} \% 7 \mathrm{D}}$ 
Figure S14. Extracted ion chromatogram, MS and MS/MS spectra of compound 14a.
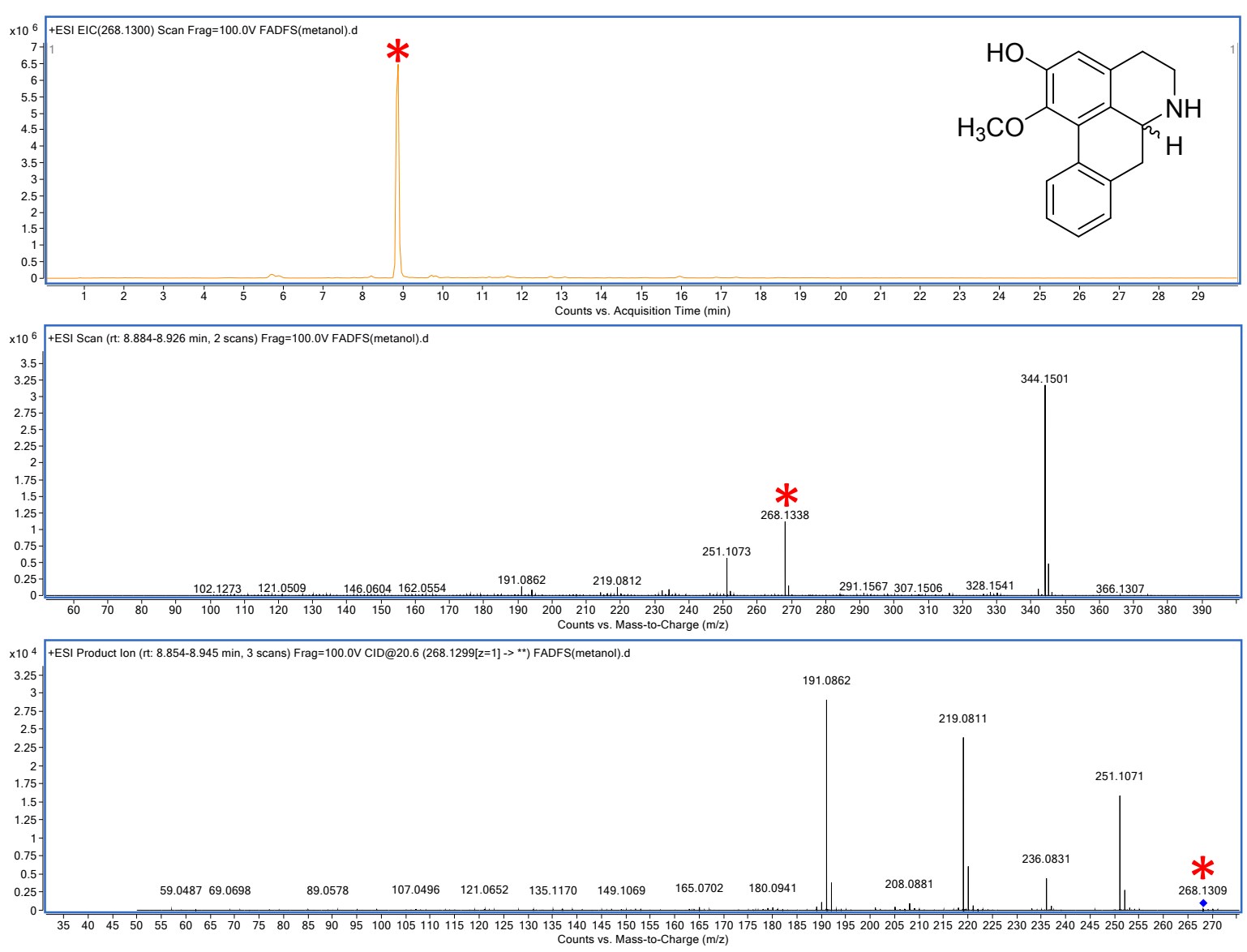

https://gnps.ucsd.edu/ProteoSAFe/gnpslibraryspectrum.jsp?SpectrumID=CCMSLIB0000543 6078\#\%7B\%7D 
Figure S15. Extracted ion chromatogram, MS and MS/MS spectra of compound $\mathbf{1 4 b}$.
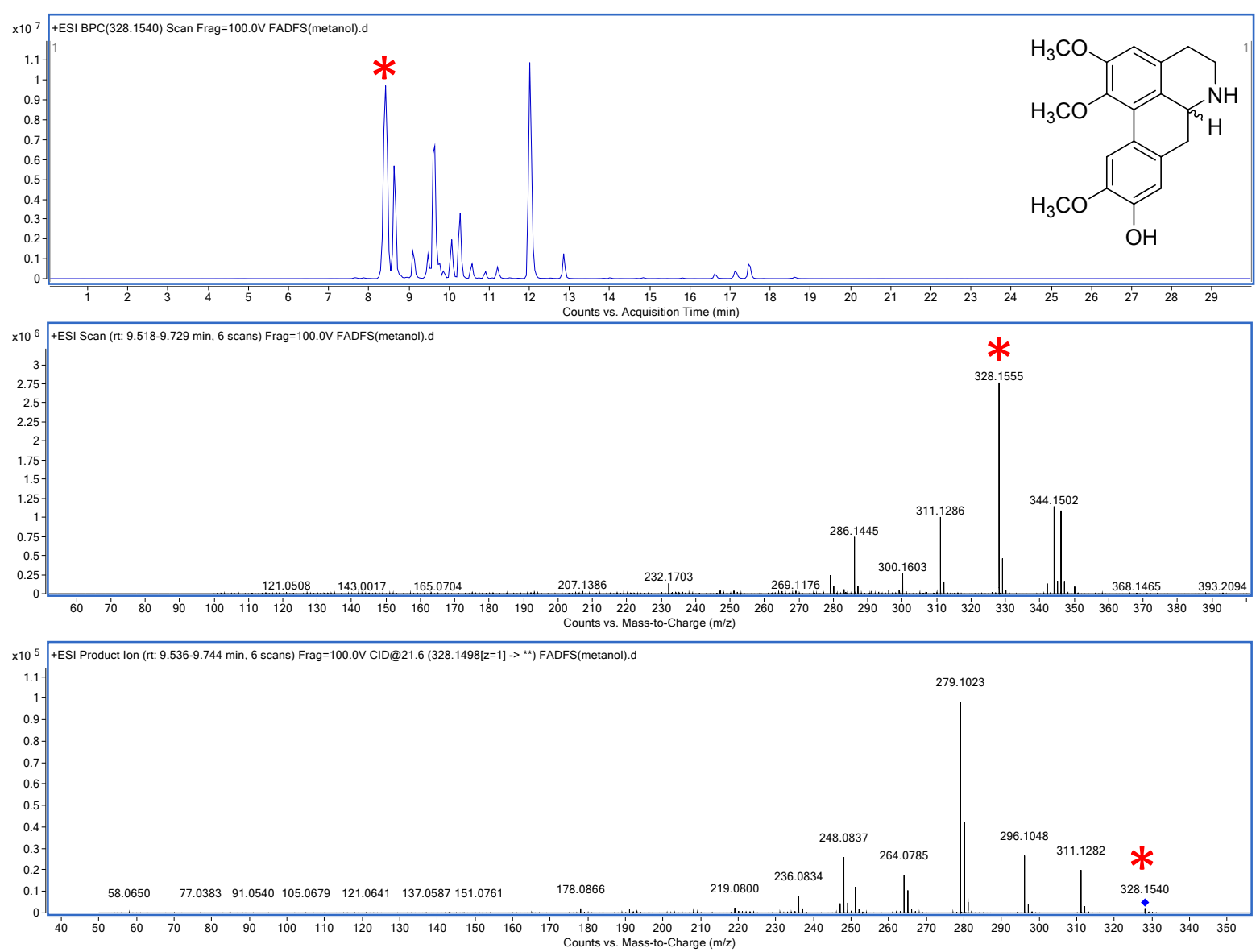

https://gnps.ucsd.edu/ProteoSAFe/gnpslibraryspectrum.jsp?SpectrumID=CCMSLIB0000543 $\underline{6056 \# \% 7 \mathrm{~B} \% 7 \mathrm{D}}$ 
Figure S16. Extracted ion chromatogram, MS and MS/MS spectra of compound 14c.
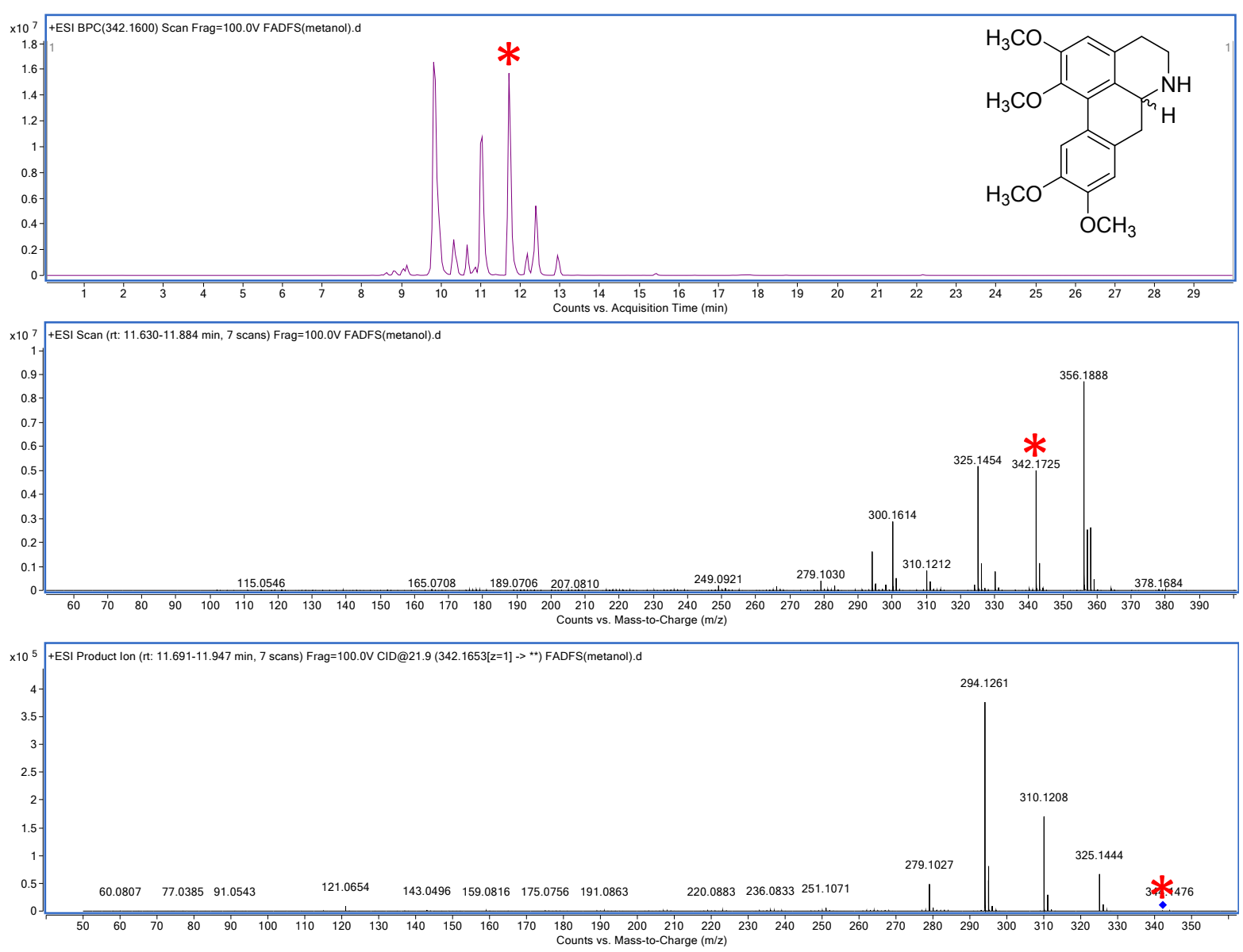

https://gnps.ucsd.edu/ProteoSAFe/gnpslibraryspectrum.jsp?SpectrumID=CCMSLIB0000543 $\underline{6057 \# \% 7 \mathrm{~B} \% 7 \mathrm{D}}$ 
Figure S17. Extracted ion chromatogram, MS and MS/MS spectra of compound 14d.
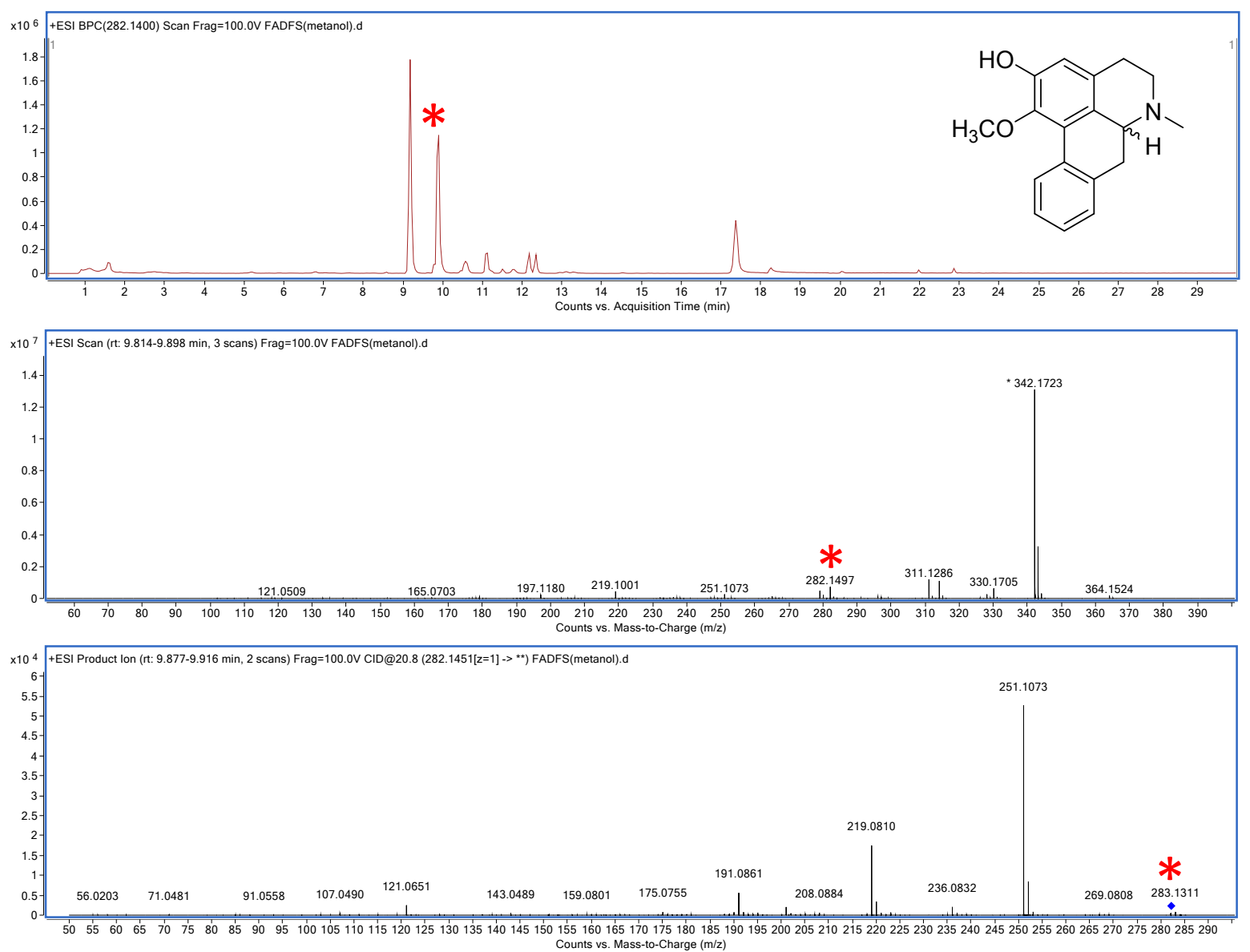

https://gnps.ucsd.edu/ProteoSAFe/gnpslibraryspectrum.jsp?SpectrumID=CCMSLIB0000543 6058\#\%7B\%7D 
Figure S18. Extracted ion chromatogram, MS and MS/MS spectra of compound 14e.
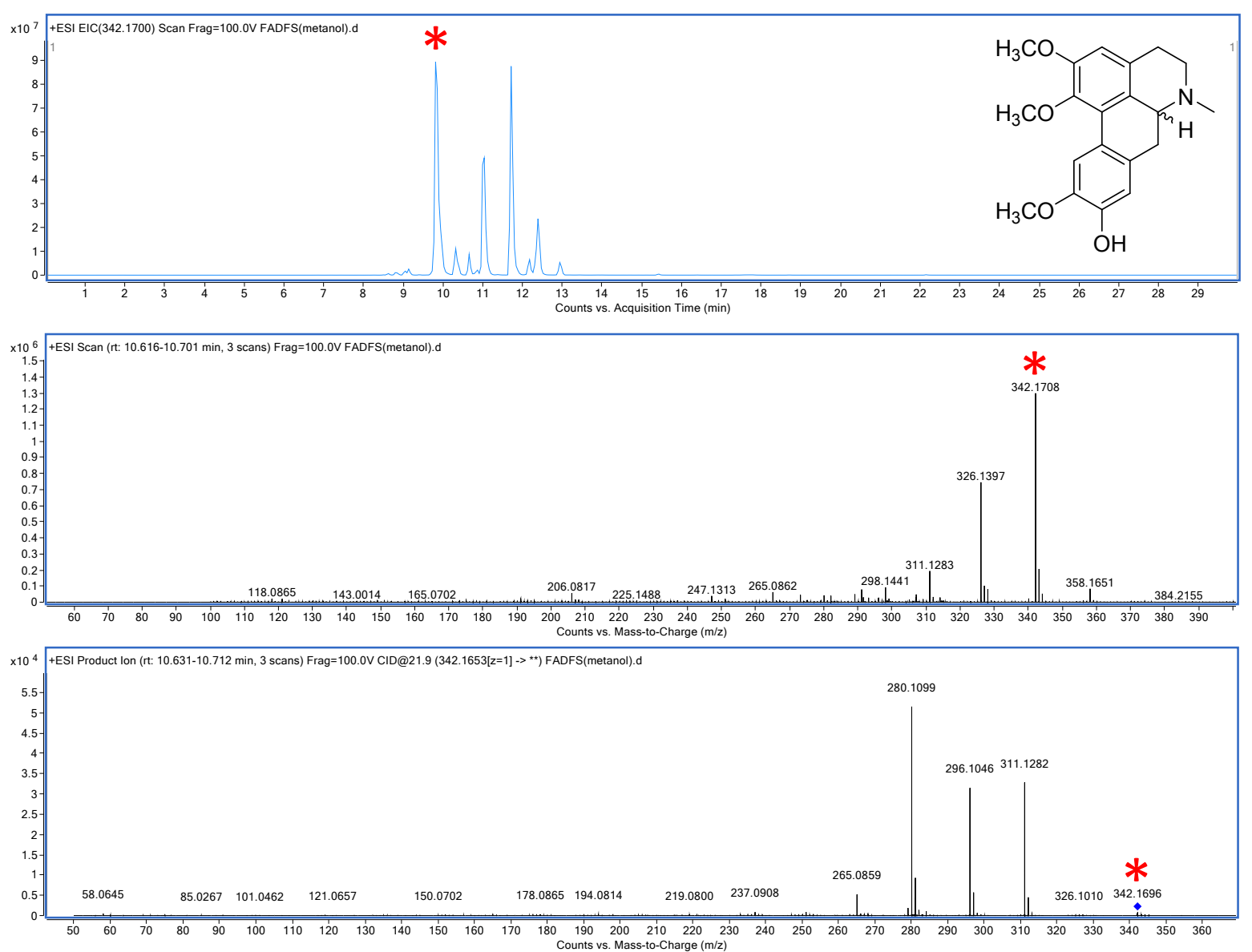

https://gnps.ucsd.edu/ProteoSAFe/gnpslibraryspectrum.jsp?SpectrumID=CCMSLIB0000543 6059\#\%7B\%7D 
Figure S19. Extracted ion chromatogram, MS and MS/MS spectra of compound $\mathbf{1 4 f}$.
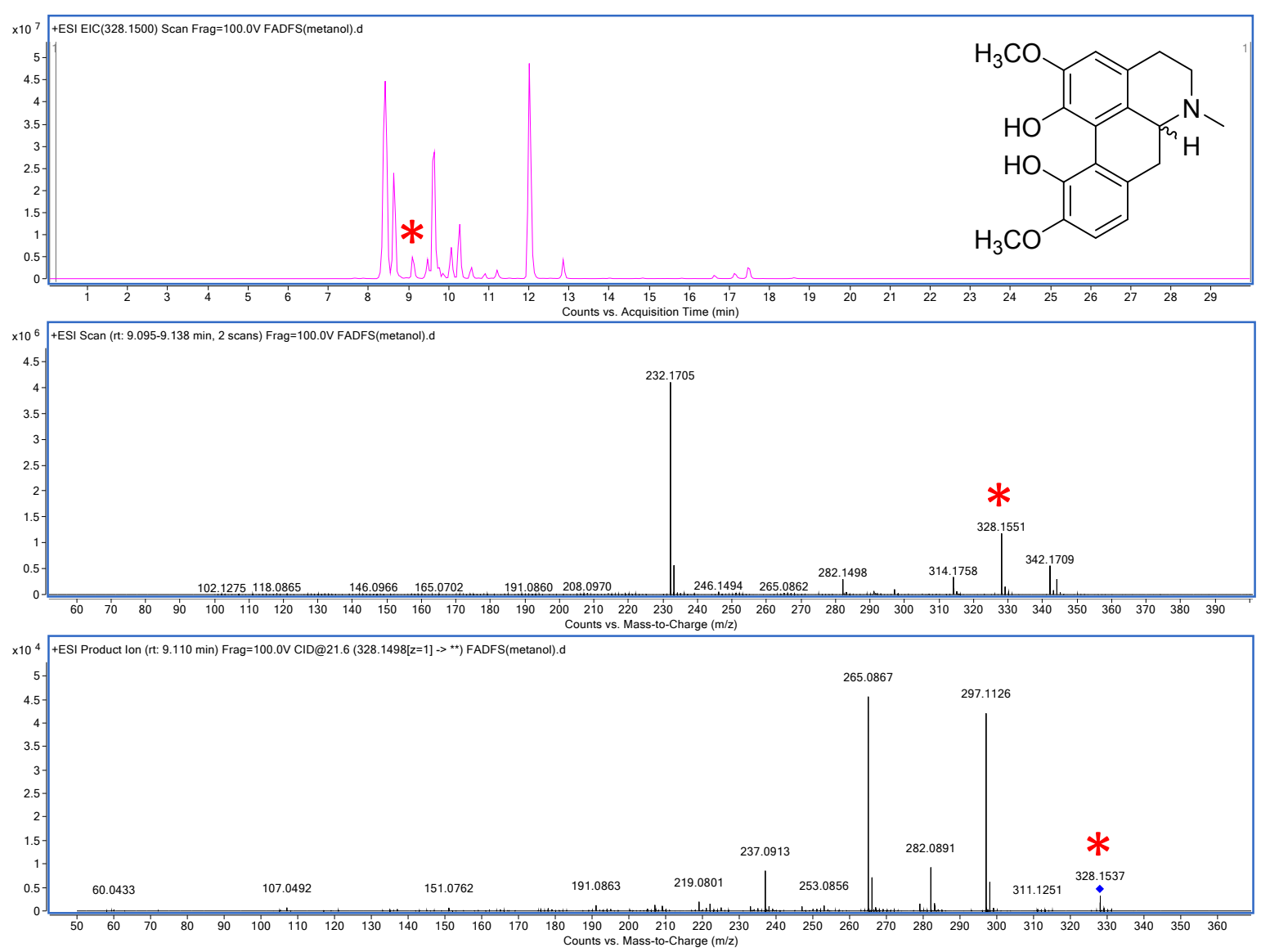

https://gnps.ucsd.edu/ProteoSAFe/gnpslibraryspectrum.jsp?SpectrumID=CCMSLIB0000543 $6060 \# \% 7 \mathrm{~B} \% 7 \mathrm{D}$ 
Figure S20. Extracted ion chromatogram, MS and MS/MS spectra of compound 14g.
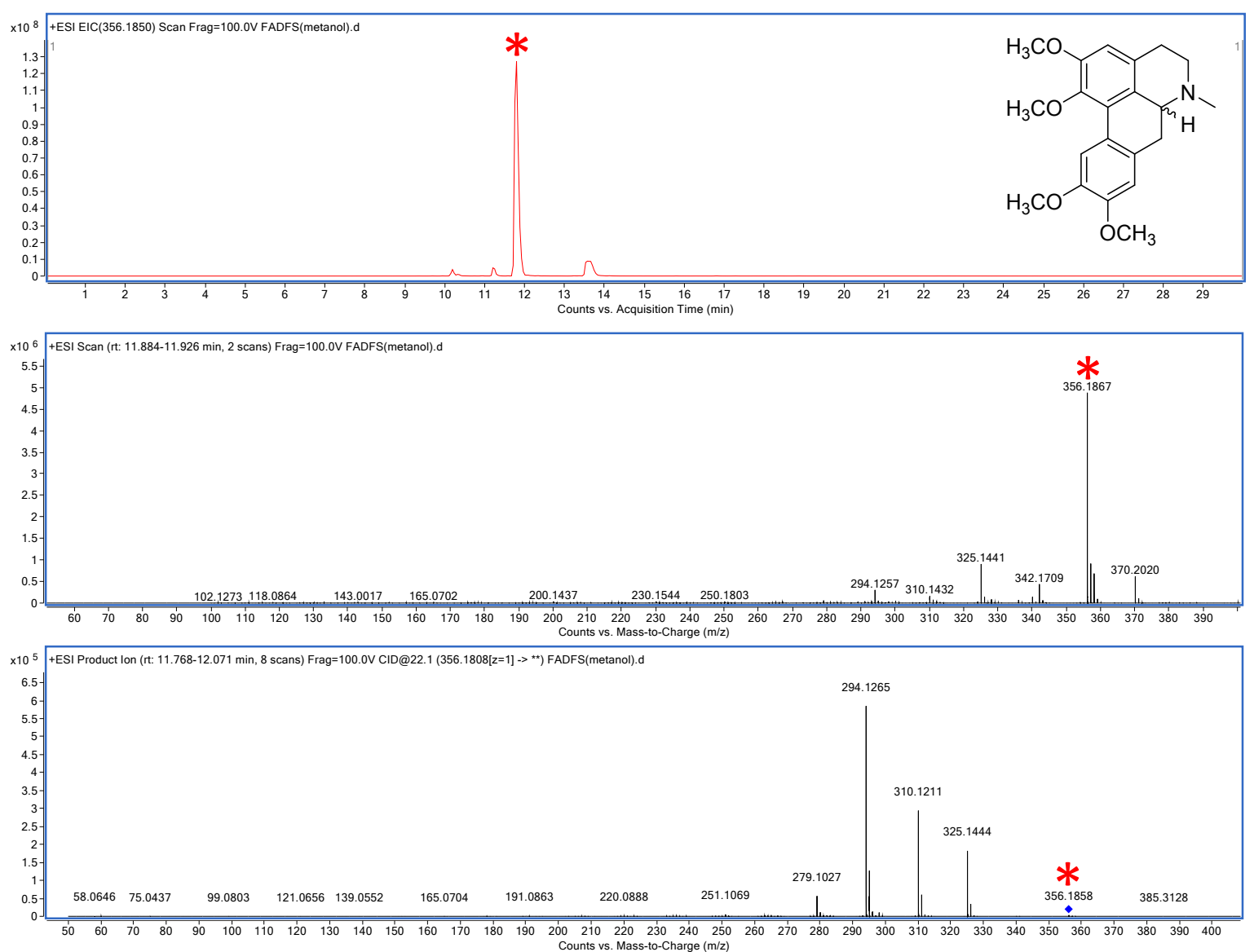

https://gnps.ucsd.edu/ProteoSAFe/gnpslibraryspectrum.jsp?SpectrumID=CCMSLIB0000543 $6061 \# \% 7 \mathrm{~B} \% 7 \mathrm{D}$ 
Figure S21. Extracted ion chromatogram, MS and MS/MS spectra of compound $\mathbf{1 4 h}$.
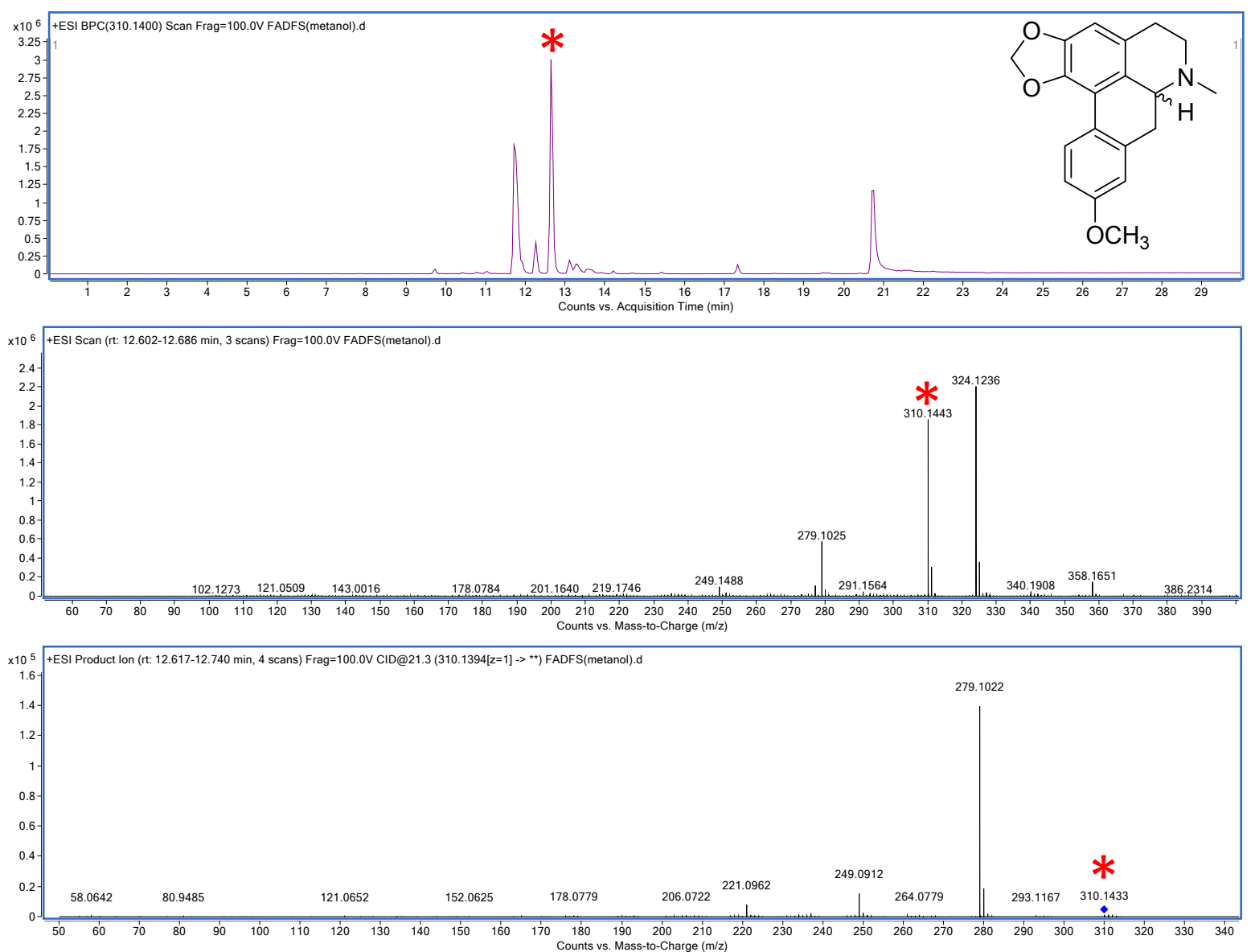

https:/gnps.ucsd.edu/ProteoSAFe/gnpslibraryspectrum.jsp?SpectrumID=CCMSLIB0000543 $\underline{6062 \# \% 7 \mathrm{~B} \% 7 \mathrm{D}}$ 
Figure S22. Extracted ion chromatogram, MS and MS/MS spectra of compound $\mathbf{1 4 i}$.
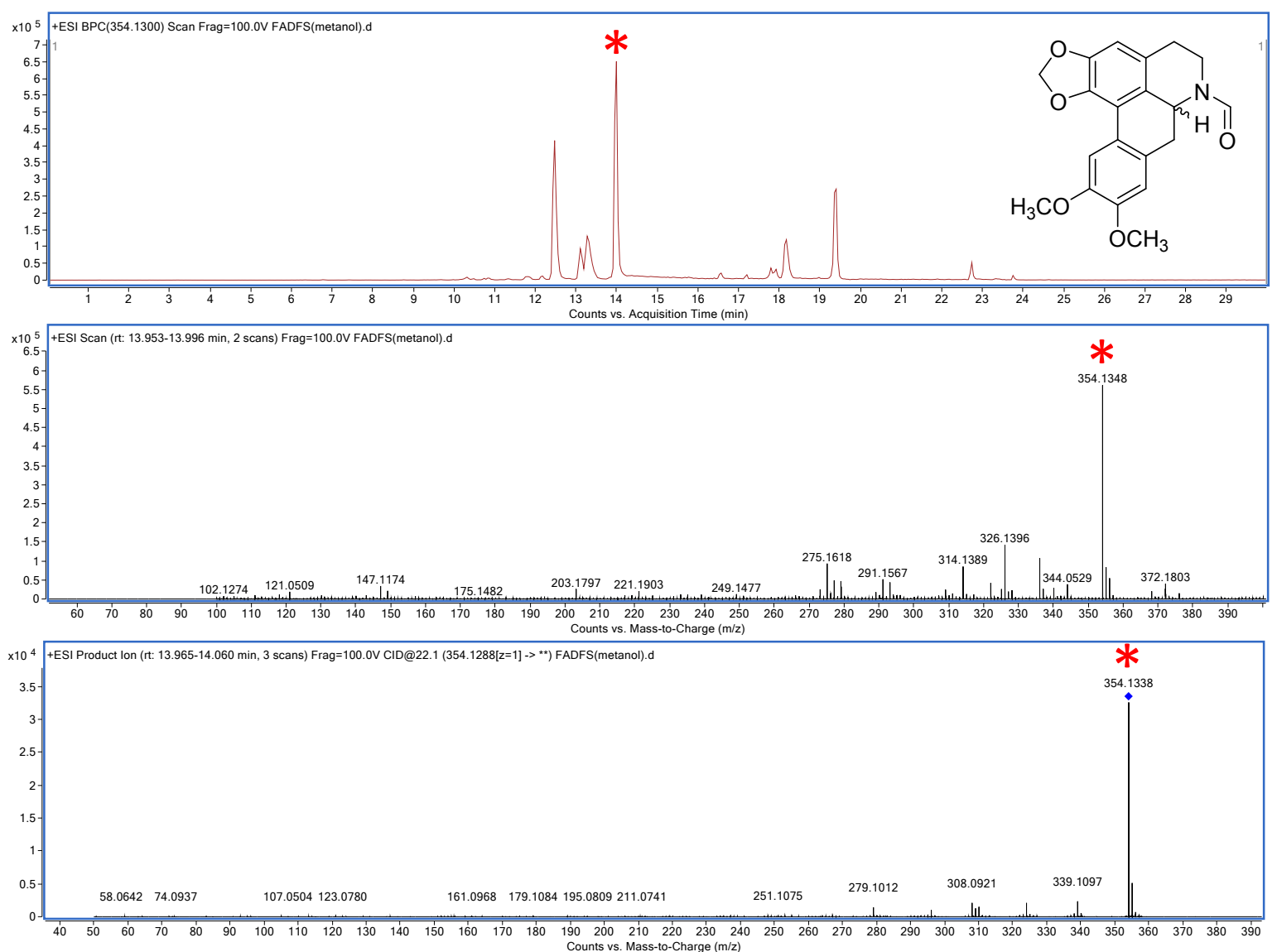

https://gnps.ucsd.edu/ProteoSAFe/gnpslibraryspectrum.jsp?SpectrumID=CCMSLIB0000543 $6063 \# \% 7 \mathrm{~B} \% 7 \mathrm{D}$ 
Figure S23. Extracted ion chromatogram, MS and MS/MS spectra of compound $\mathbf{1 4 j}$.
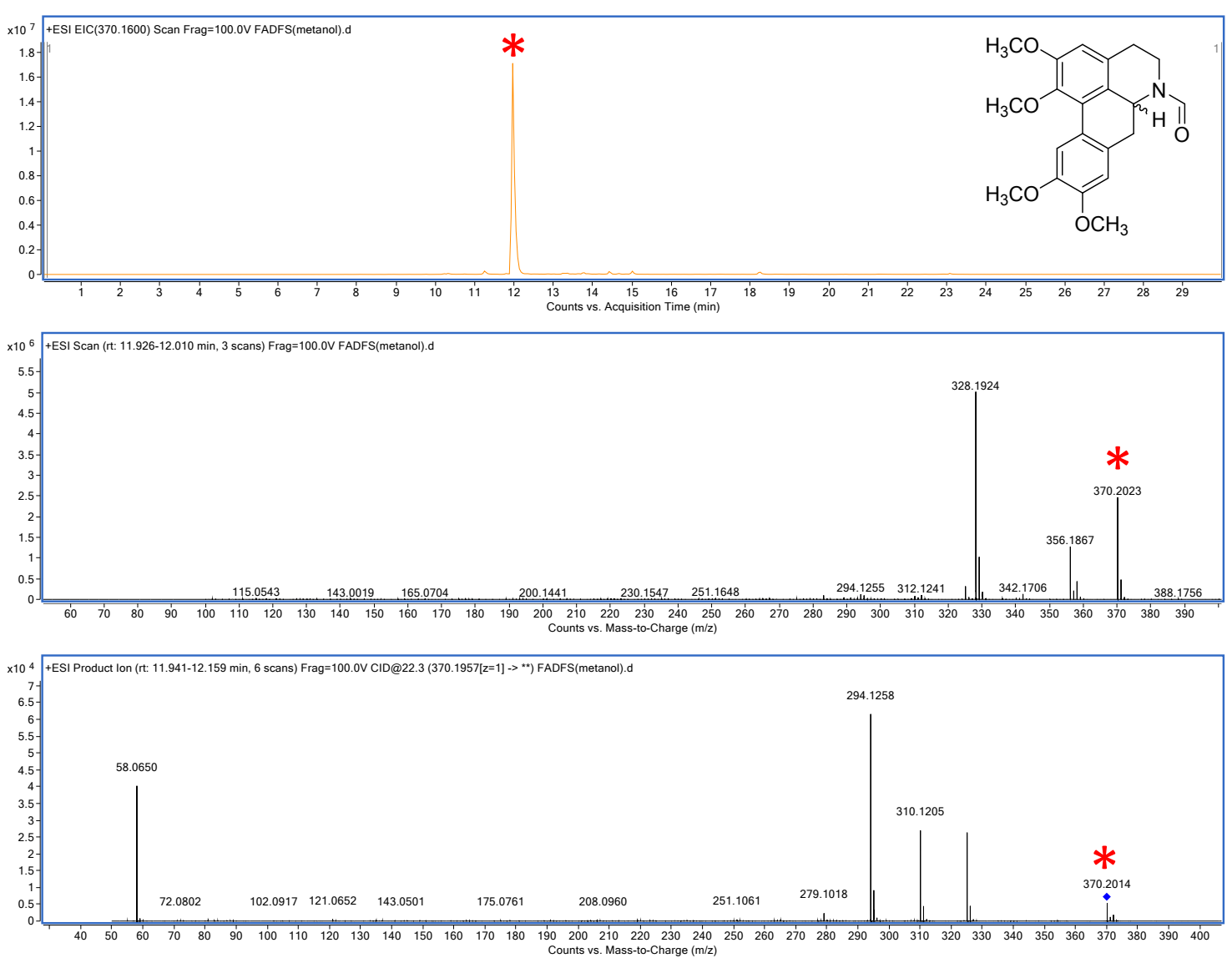

https://gnps.ucsd.edu/ProteoSAFe/gnpslibraryspectrum.jsp?SpectrumID=CCMSLIB0000543 $\underline{6064 \# \% 7 \mathrm{~B} \% 7 \mathrm{D}}$ 
Figure S24. Extracted ion chromatogram, MS and MS/MS spectra of compound 14k.
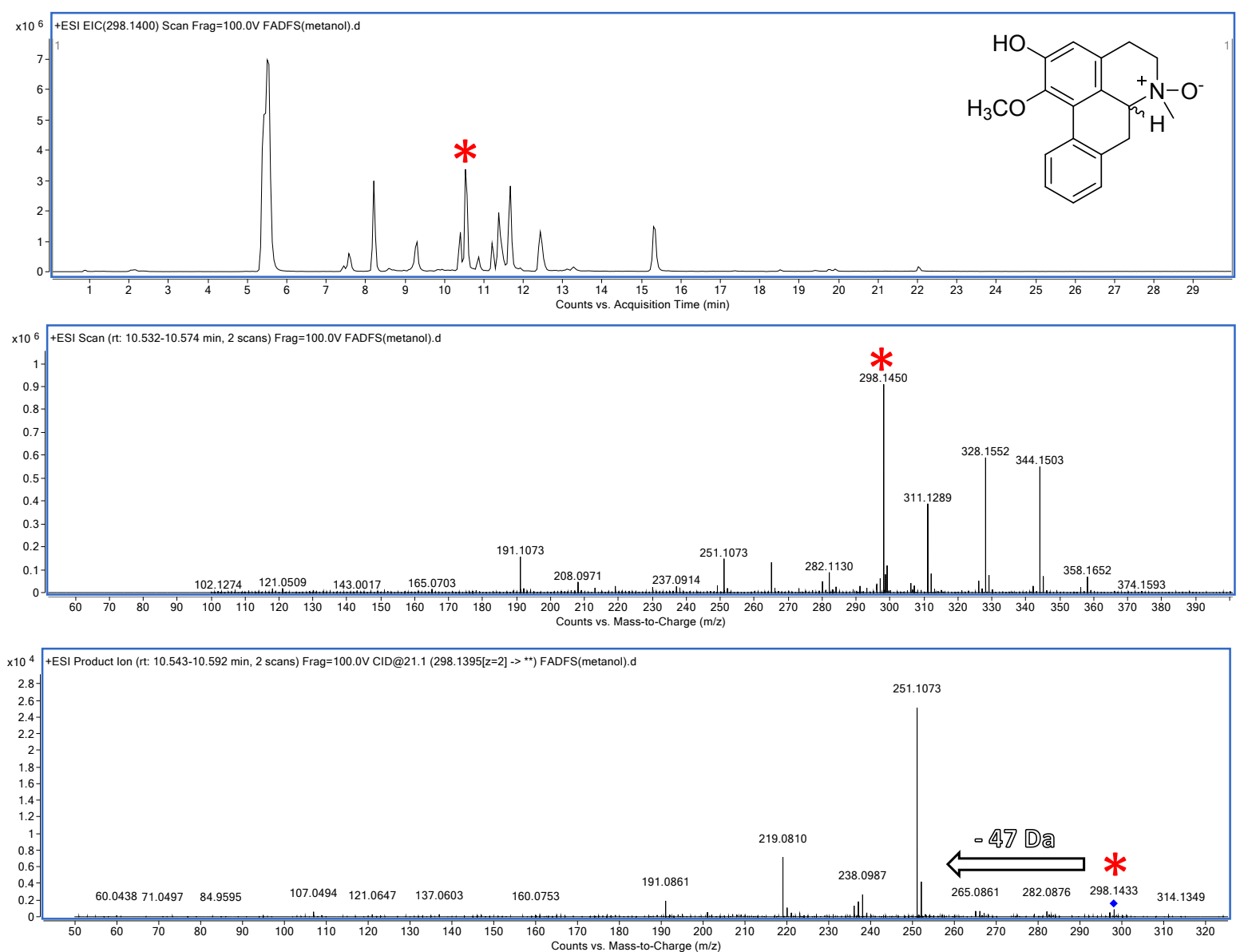

https://gnps.ucsd.edu/ProteoSAFe/gnpslibraryspectrum.jsp?SpectrumID=CCMSLIB0000543 $\underline{6065 \# \% 7 \mathrm{~B} \% 7 \mathrm{D}}$ 
Figure S25. Extracted ion chromatogram, MS and MS/MS spectra of compound $\mathbf{1 4 1 .}$
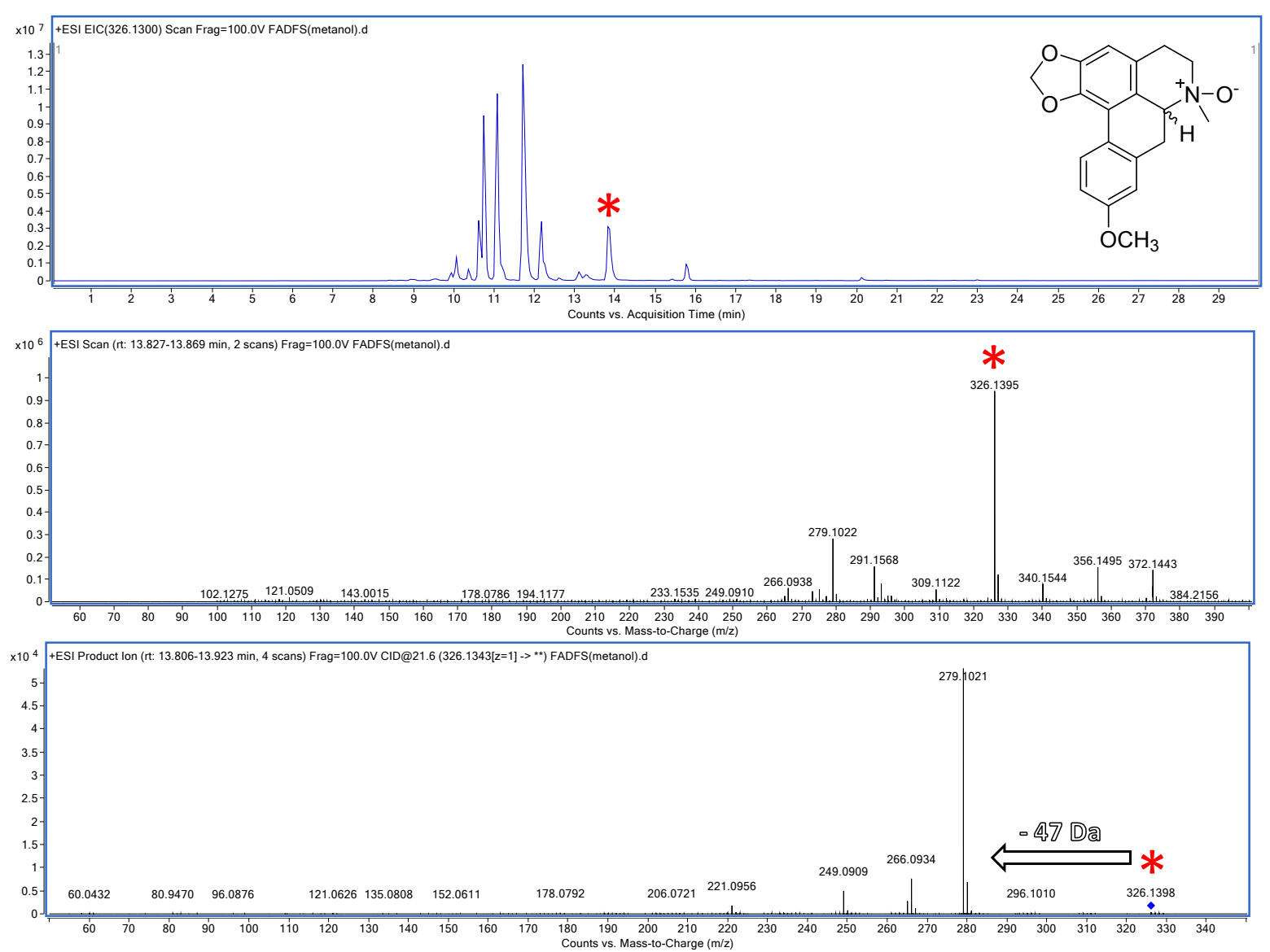

https://gnps.ucsd.edu/ProteoSAFe/gnpslibraryspectrum.jsp?SpectrumID=CCMSLIB0000543 $\underline{6066 \# \% 7 \mathrm{~B} \% 7 \mathrm{D}}$ 
Figure S26. Extracted ion chromatogram, MS and MS/MS spectra of compound $\mathbf{1 4 m}$.
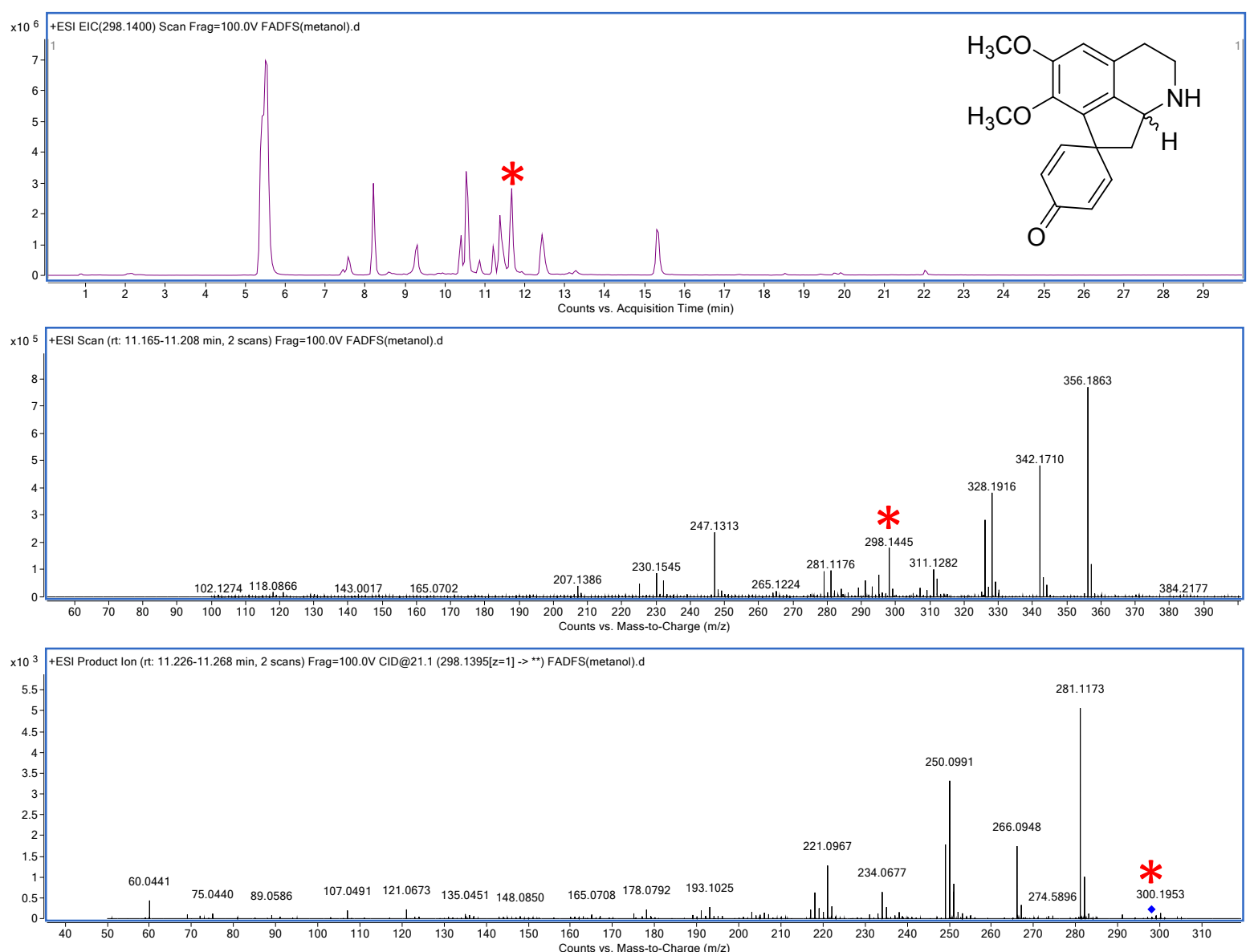

https://gnps.ucsd.edu/ProteoSAFe/gnpslibraryspectrum.jsp?SpectrumID=CCMSLIB0000543 $\underline{6067 \# \% 7 \mathrm{~B} \% 7 \mathrm{D}}$ 
Figure S27. Extracted ion chromatogram, MS and MS/MS spectra of compound 15.
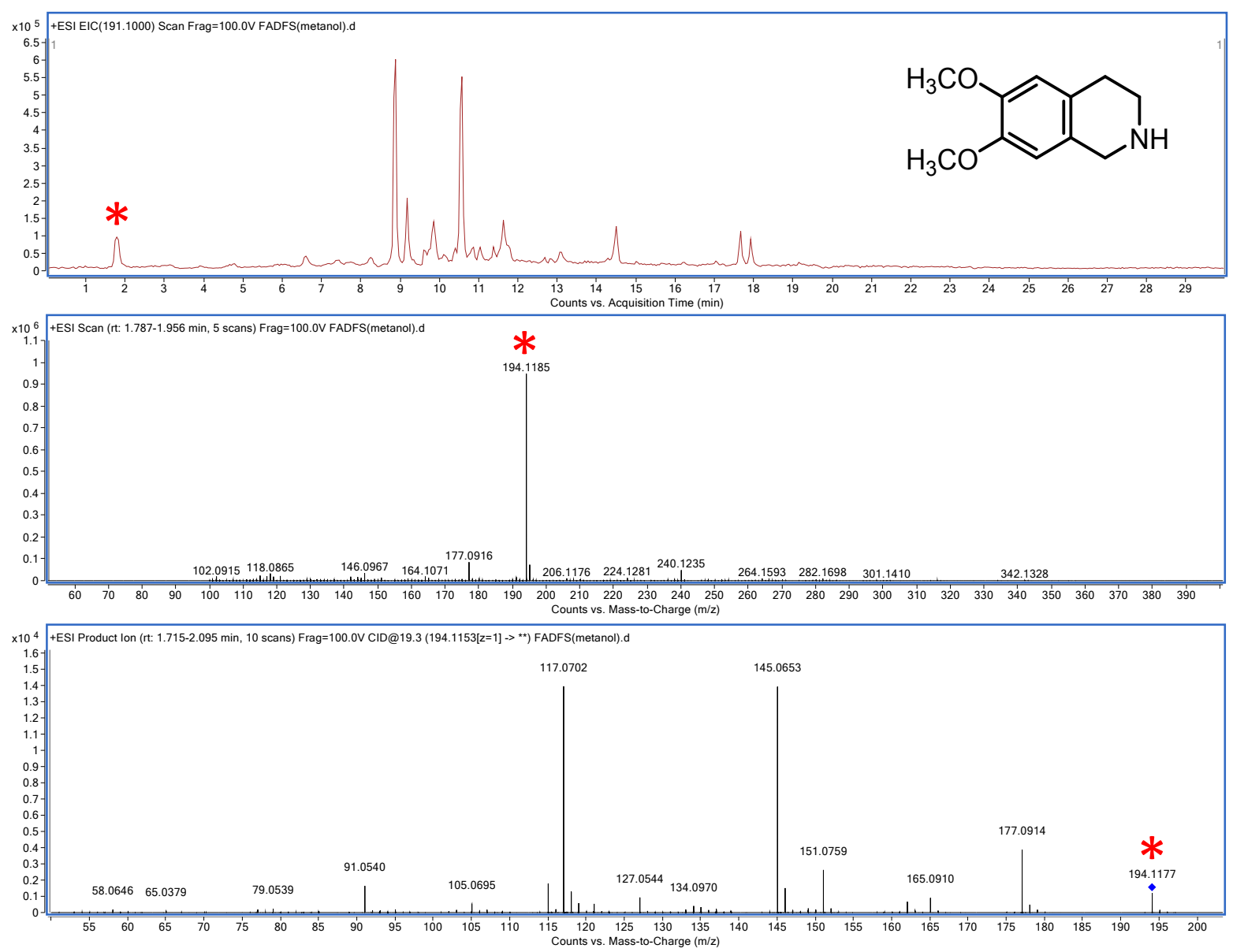

https://gnps.ucsd.edu/ProteoSAFe/gnpslibraryspectrum.jsp?SpectrumID=CCMSLIB0000543 $\underline{6068 \# \% 7 \mathrm{~B} \% 7 \mathrm{D}}$ 
Figure S28. ${ }^{1} \mathrm{H}$ NMR spectrum of compound 3 in $\mathrm{CDCl}_{3}$ at $500 \mathrm{MHz}$.

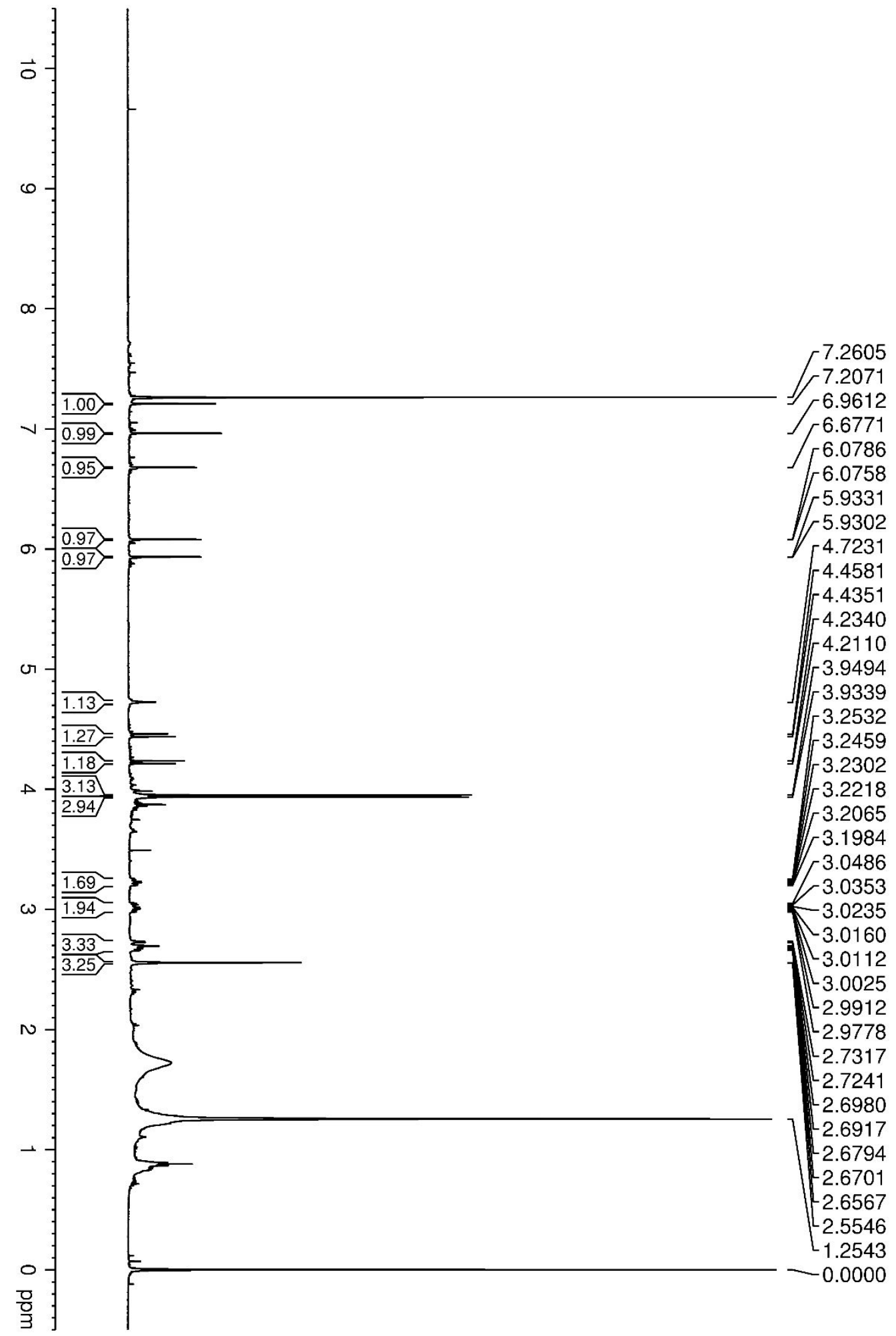


Figure S29. Enlargement ( $\delta$ 7.20-5.93) of ${ }^{1} \mathrm{H}$ NMR spectrum of compound 3 in $\mathrm{CDCl}_{3}$ at 500 $\mathrm{MHz}$.

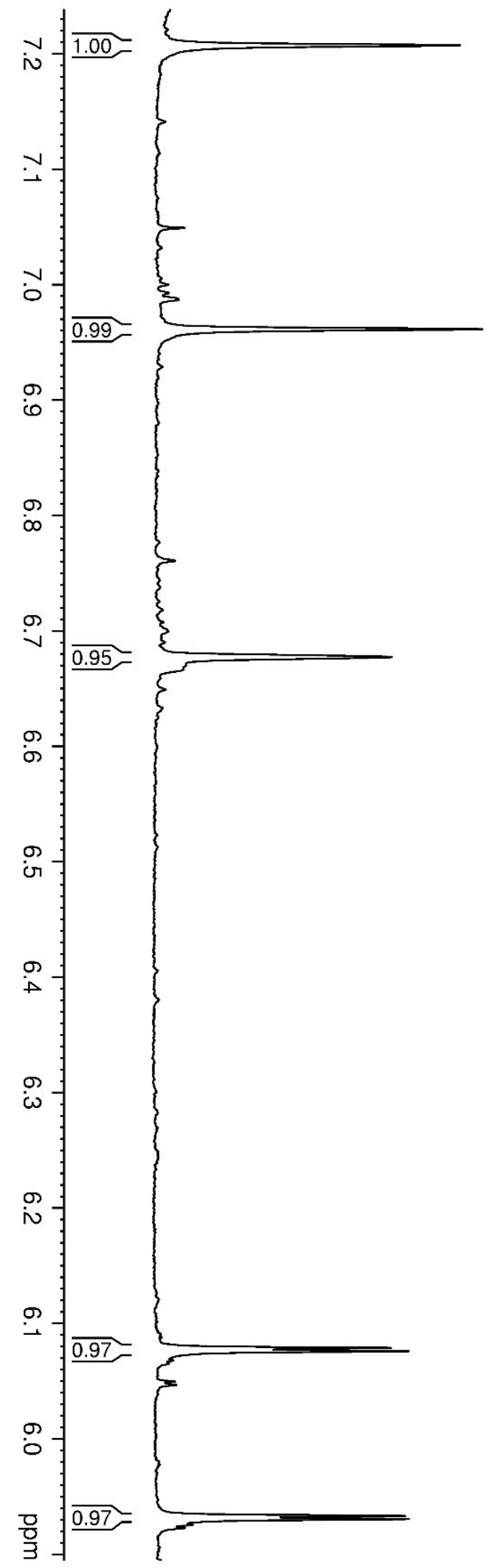


Figure S30. Enlargement $\left(\delta\right.$ 4.72-3.93) of ${ }^{1} \mathrm{H}$ NMR spectrum of compound 3 in $\mathrm{CDCl}_{3}$ at 500 $\mathrm{MHz}$.

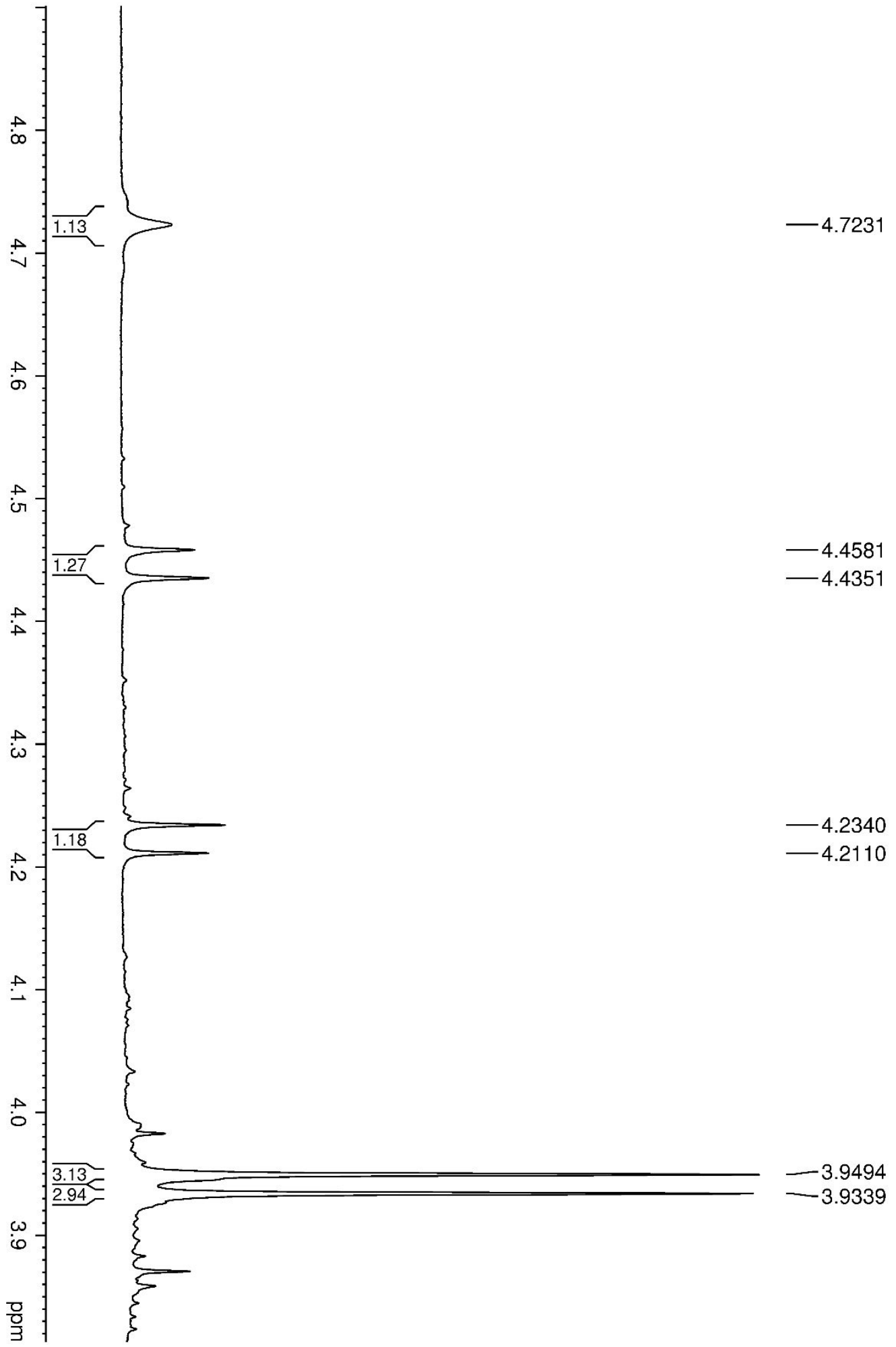


Figure S31. Enlargement $(\delta 3.25-2.55)$ of ${ }^{1} \mathrm{H}$ NMR spectrum of compound 3 in $\mathrm{CDCl}_{3}$ at 500 $\mathrm{MHz}$.

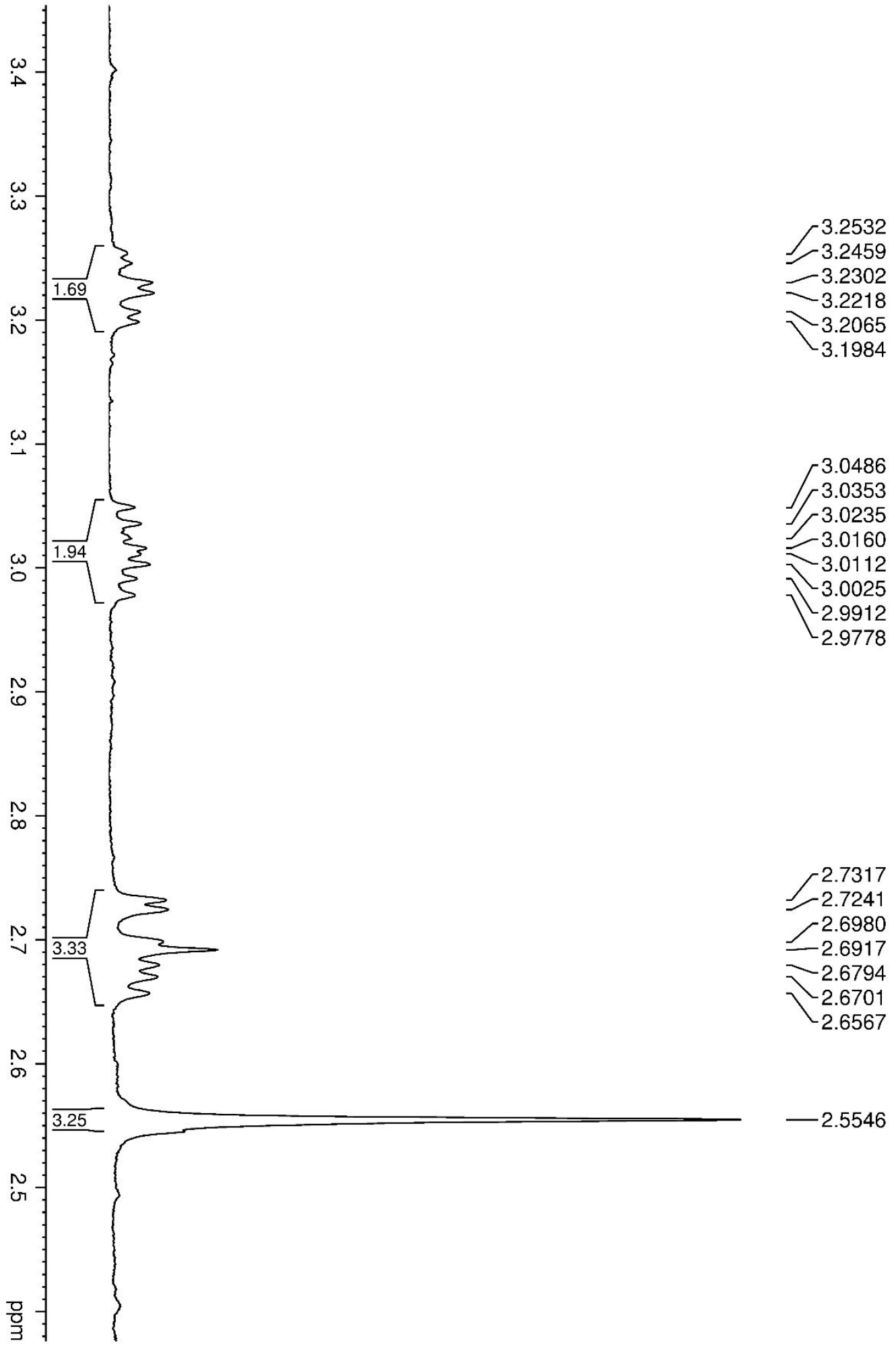


Figure S32. Enlargement ( $\delta 3.25-2.97)$ of ${ }^{1} \mathrm{H}$ NMR spectrum of compound 3 in $\mathrm{CDCl}_{3}$ at 500 $\mathrm{MHz}$.

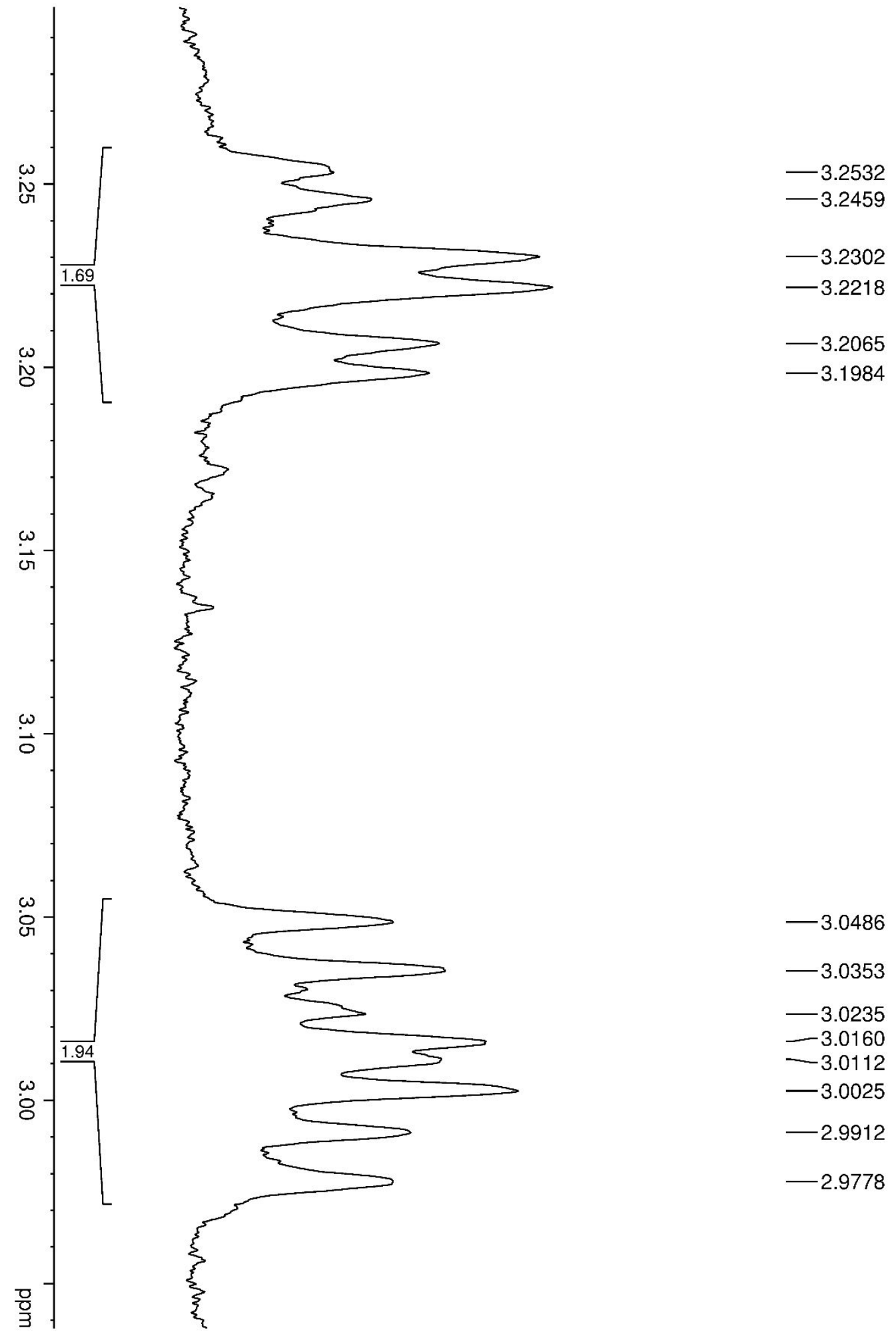


Figure S33. Enlargement $(\delta 2.73-2.65)$ of ${ }^{1} \mathrm{H}$ NMR spectrum of compound 3 in $\mathrm{CDCl}_{3}$ at 500 $\mathrm{MHz}$.

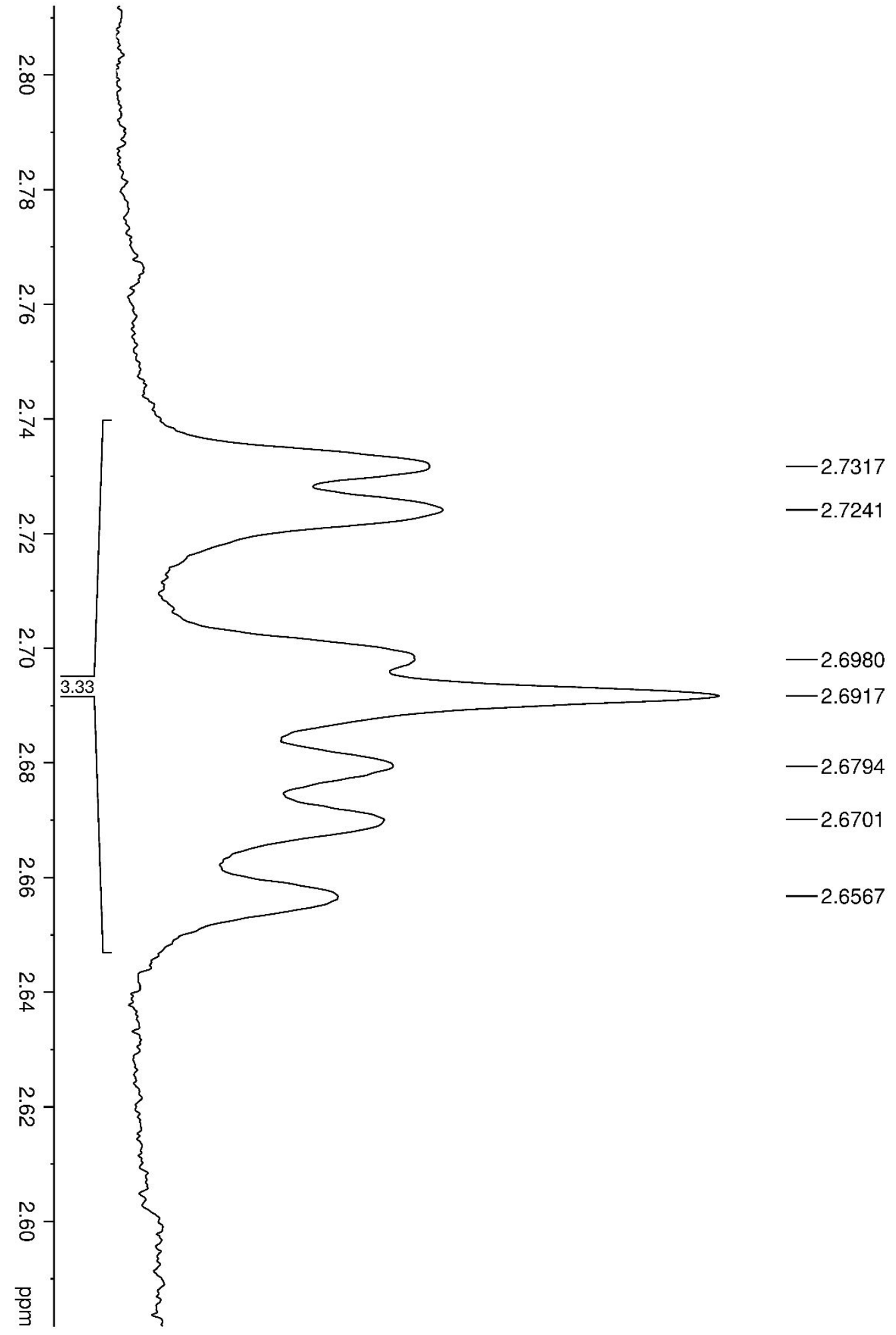


Figure 34. ${ }^{13} \mathrm{C}$ NMR spectrum of compound 3 in $\mathrm{CDCl}_{3}$ at $125 \mathrm{MHz}$.

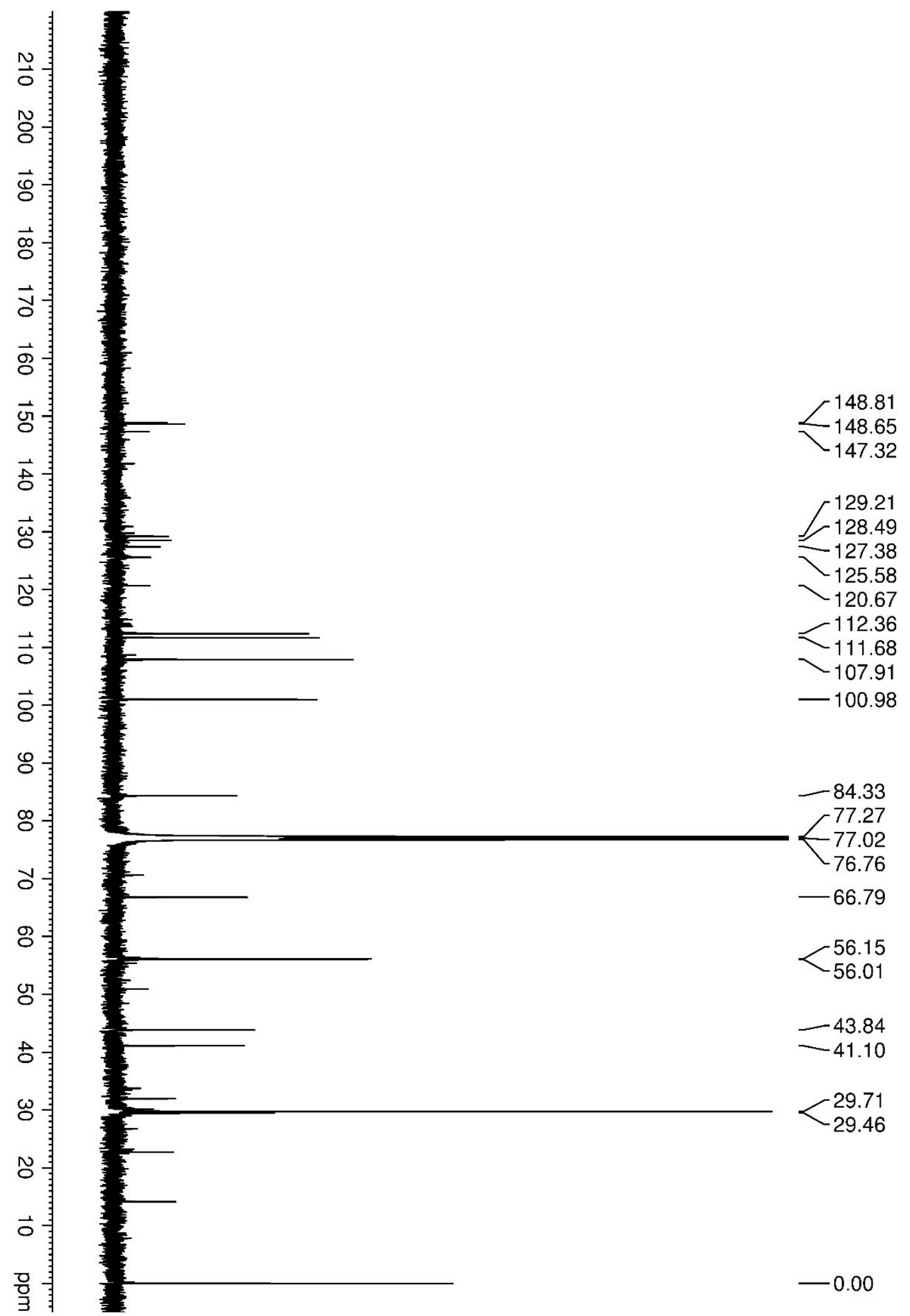


Figure S35. Enlargement ( $\delta$ 148.8-100.9) of ${ }^{13} \mathrm{C}$ NMR spectrum of compound 3 in $\mathrm{CDCl}_{3}$ at $500 \mathrm{MHz}$.

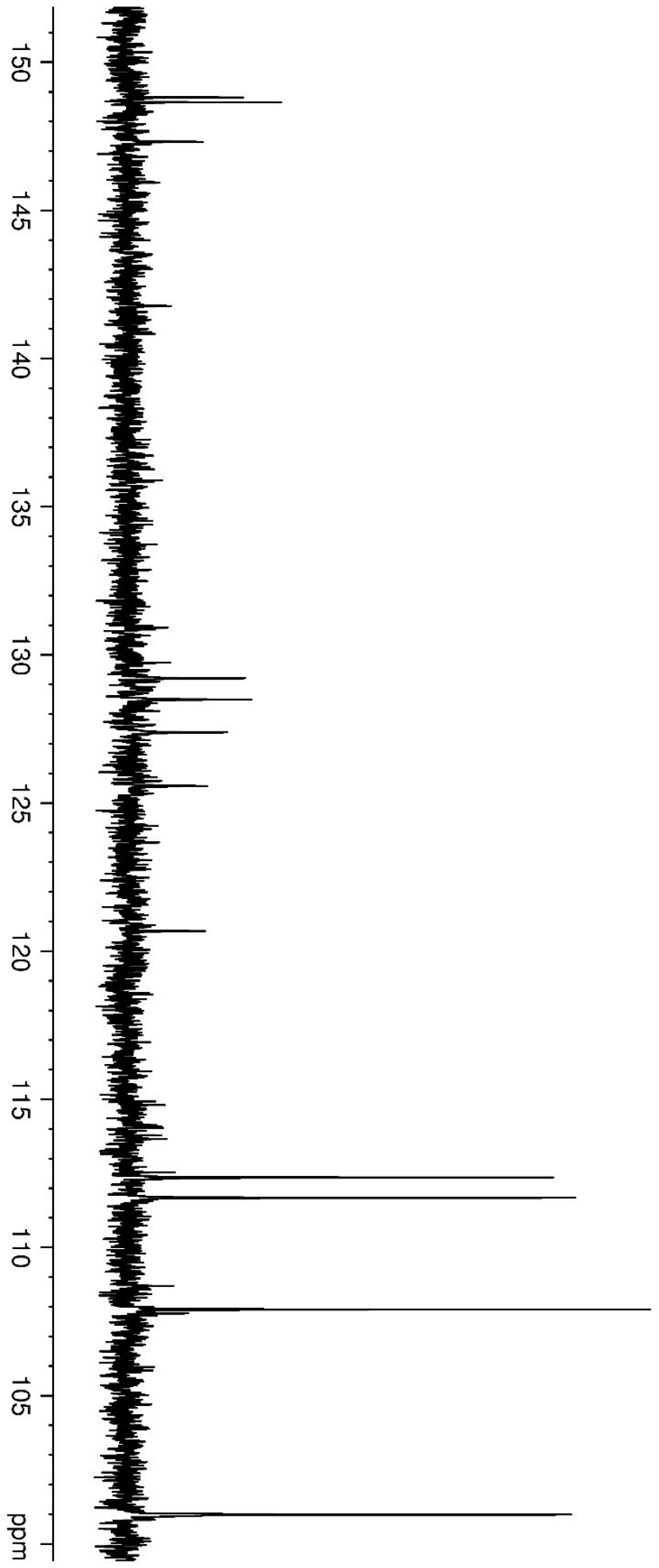

$-112.36$ 
Figure S36. Enlargement ( $\delta 66.7-29.4)$ of ${ }^{13} \mathrm{C}$ NMR spectrum of compound 3 in $\mathrm{CDCl}_{3}$ at 500 $\mathrm{MHz}$.

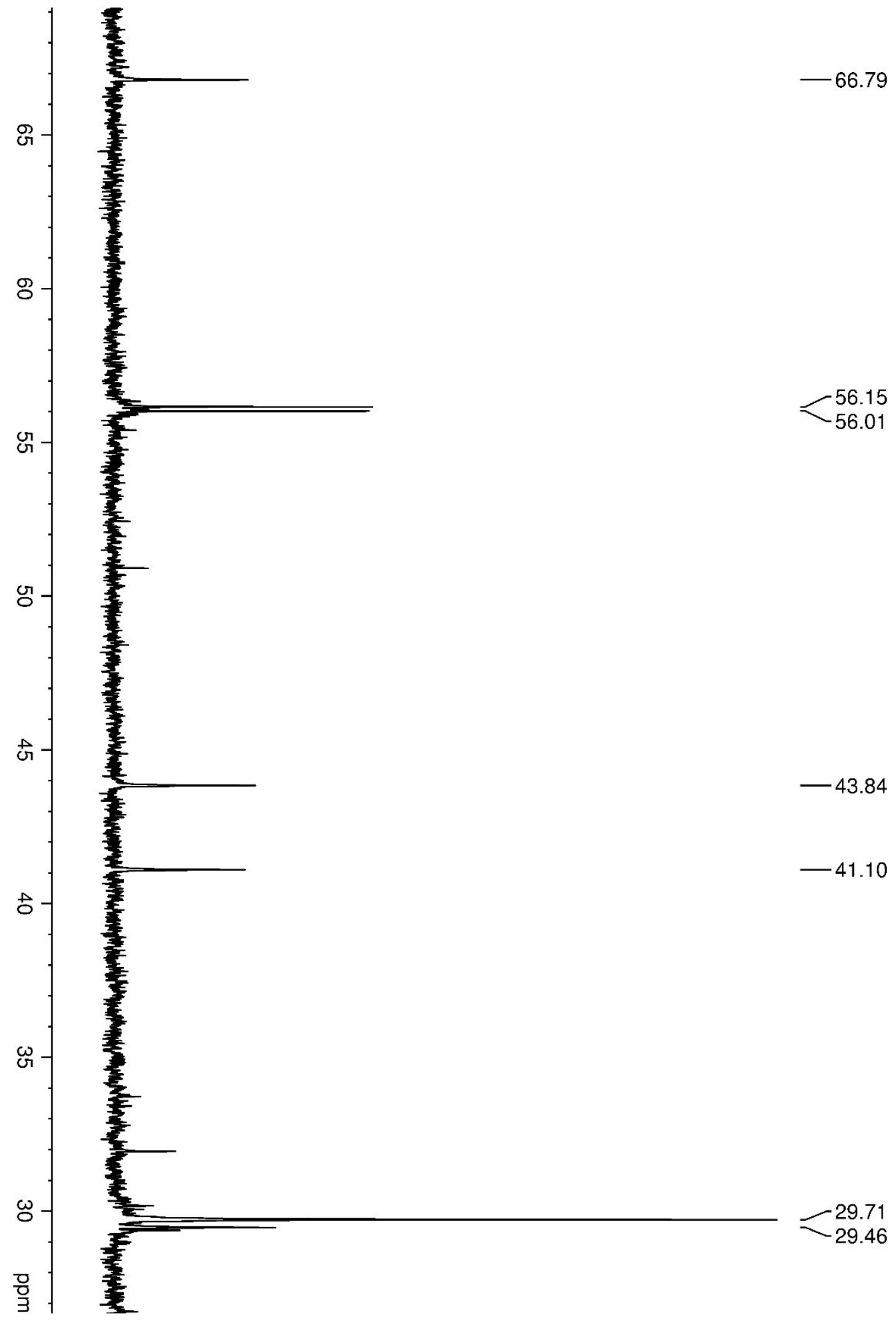


Figure S37. ${ }^{13} \mathrm{C}$ NMR DEP135 spectrum of compound 3 in $\mathrm{CDCl}_{3}$ at $125 \mathrm{MHz}$.

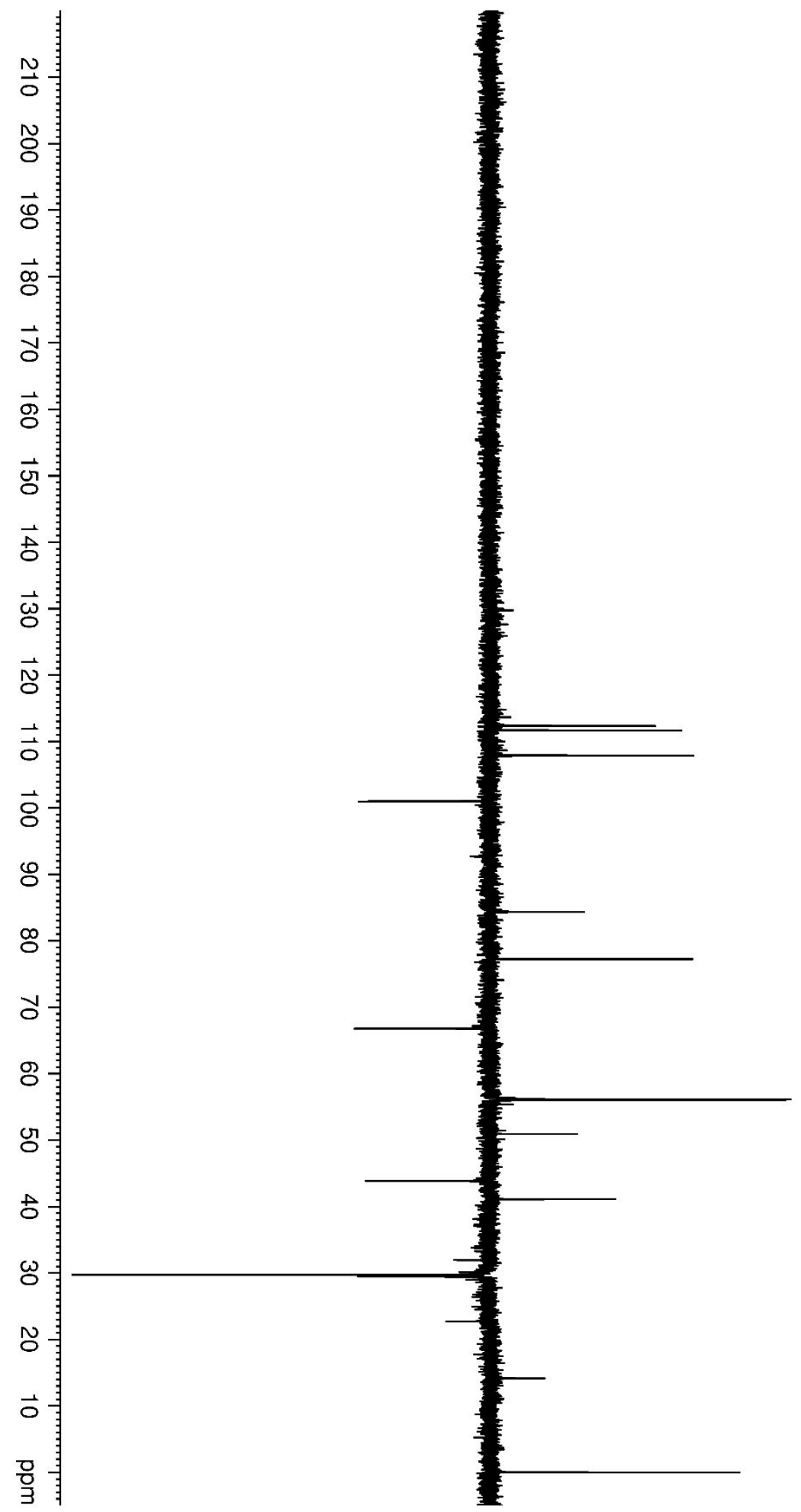


Figure S38. Enlargement $(\delta 112.3-84.3)$ of ${ }^{13} \mathrm{C}$ NMR DEP135 spectrum of compound 3 in $\mathrm{CDCl}_{3}$ at $125 \mathrm{MHz}$.

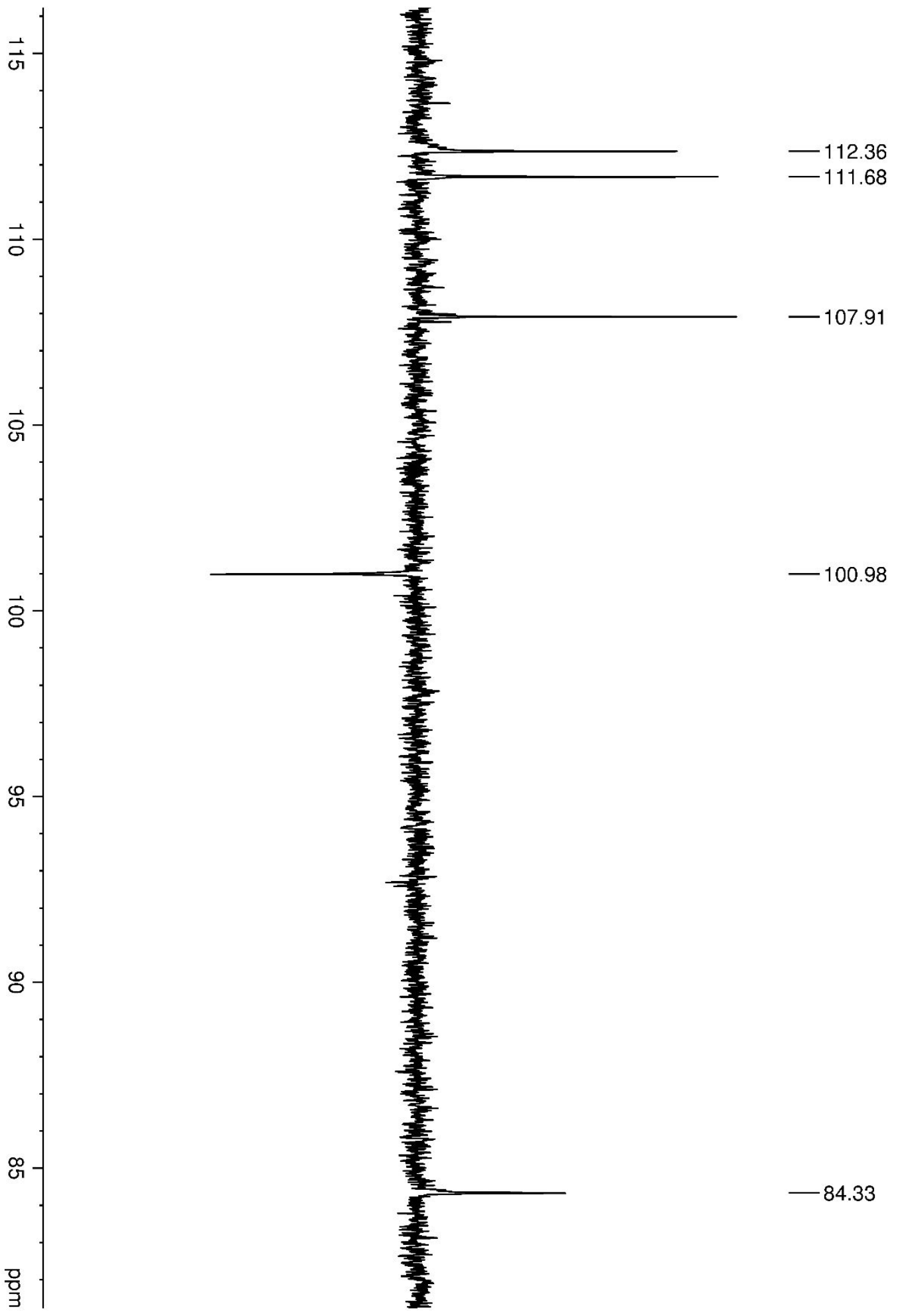


Figure S39. Enlargement $(\delta 66.7-29.4)$ of ${ }^{13} \mathrm{C}$ NMR DEP135 spectrum of compound 3 in $\mathrm{CDCl}_{3}$ at $125 \mathrm{MHz}$.

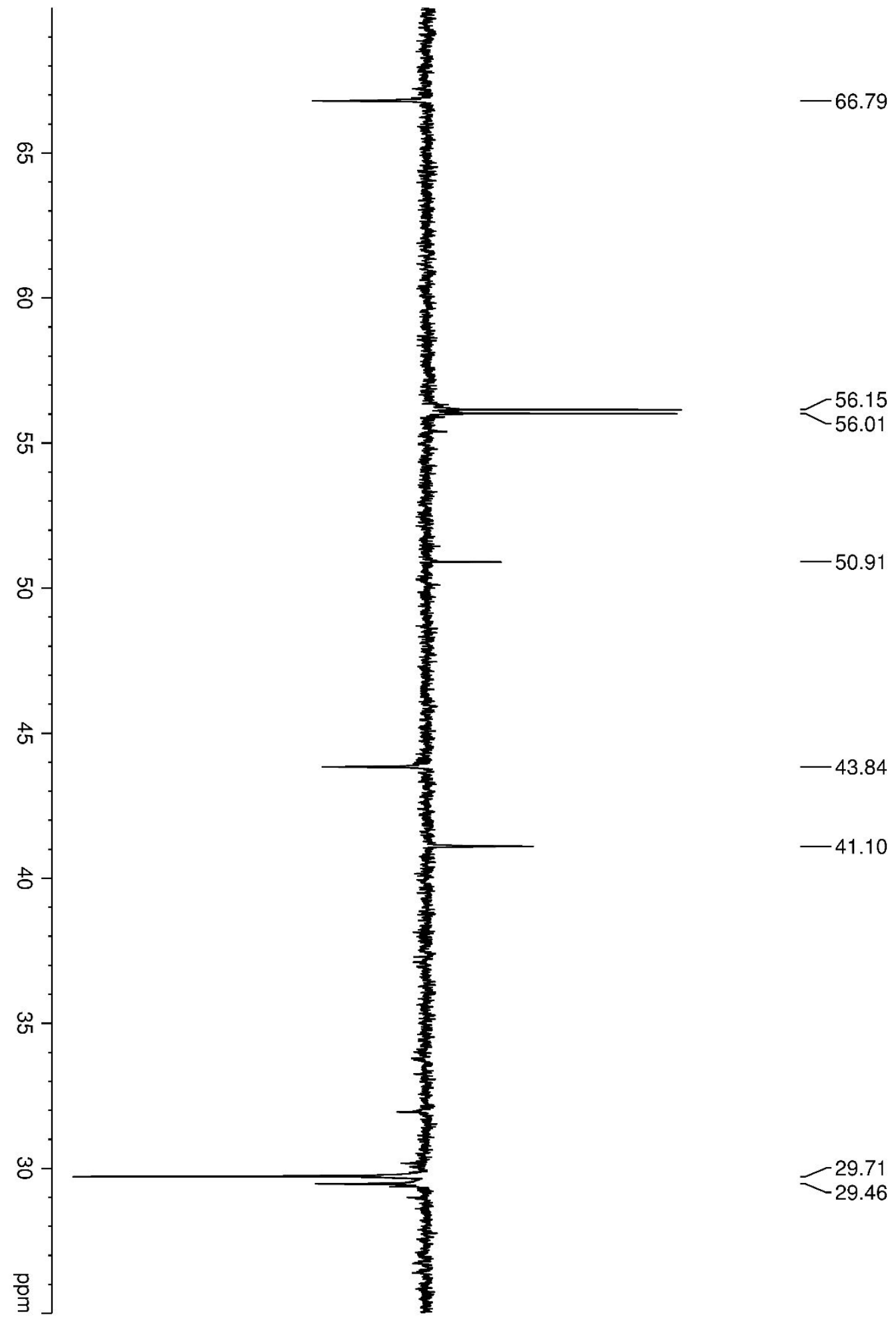


Figure S40. ${ }^{1} \mathrm{H}-{ }^{1} \mathrm{H}$ COSY correlation map from ${ }^{1} \mathrm{H}$ NMR experiment of compound 3 in $\mathrm{CDCl}_{3}$ at $500 \mathrm{MHz}$.

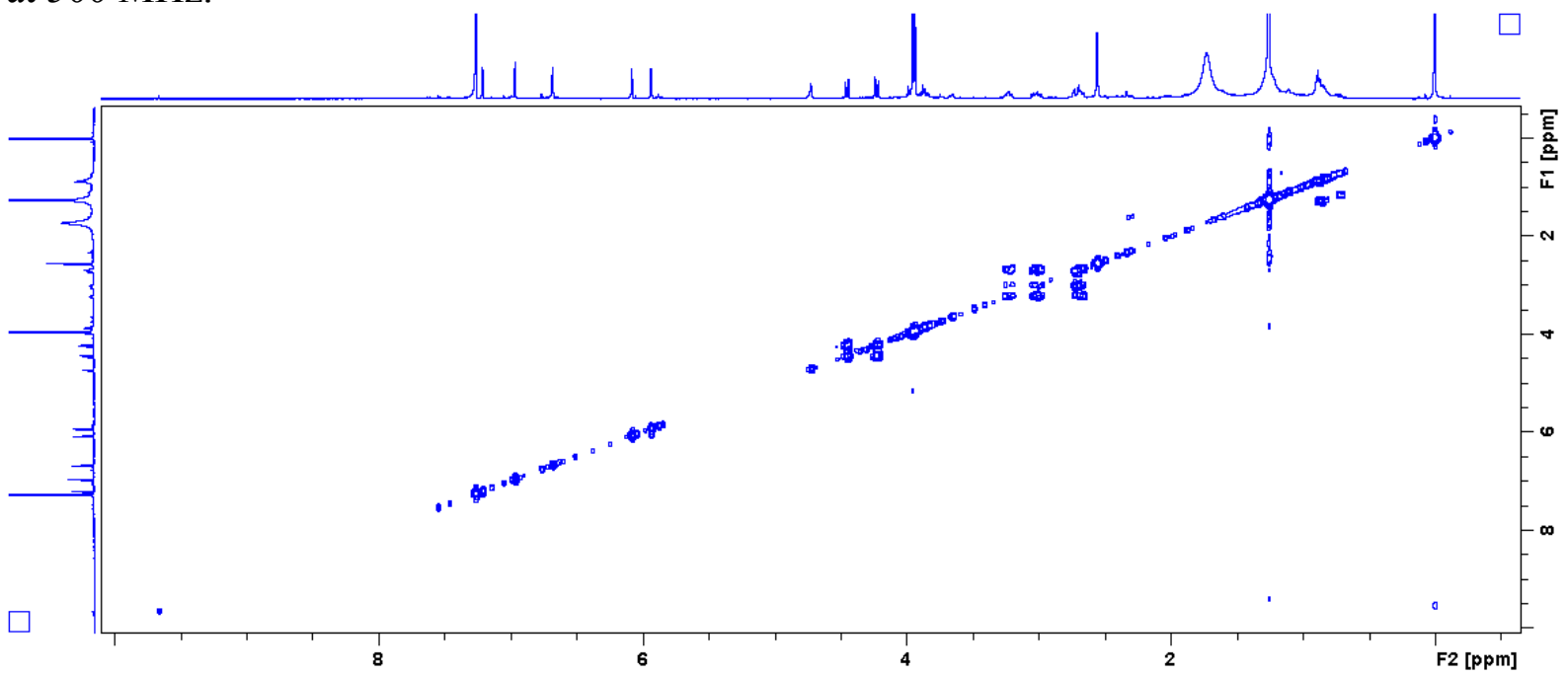

Figure S41. Enlargement $\left(\delta\right.$ 4.72-3.83) of ${ }^{1} \mathrm{H}-{ }^{1} \mathrm{H}$ COSY correlation map from ${ }^{1} \mathrm{H}$ NMR experiment of compound 3 in $\mathrm{CDCl}_{3}$ at $500 \mathrm{MHz}$.

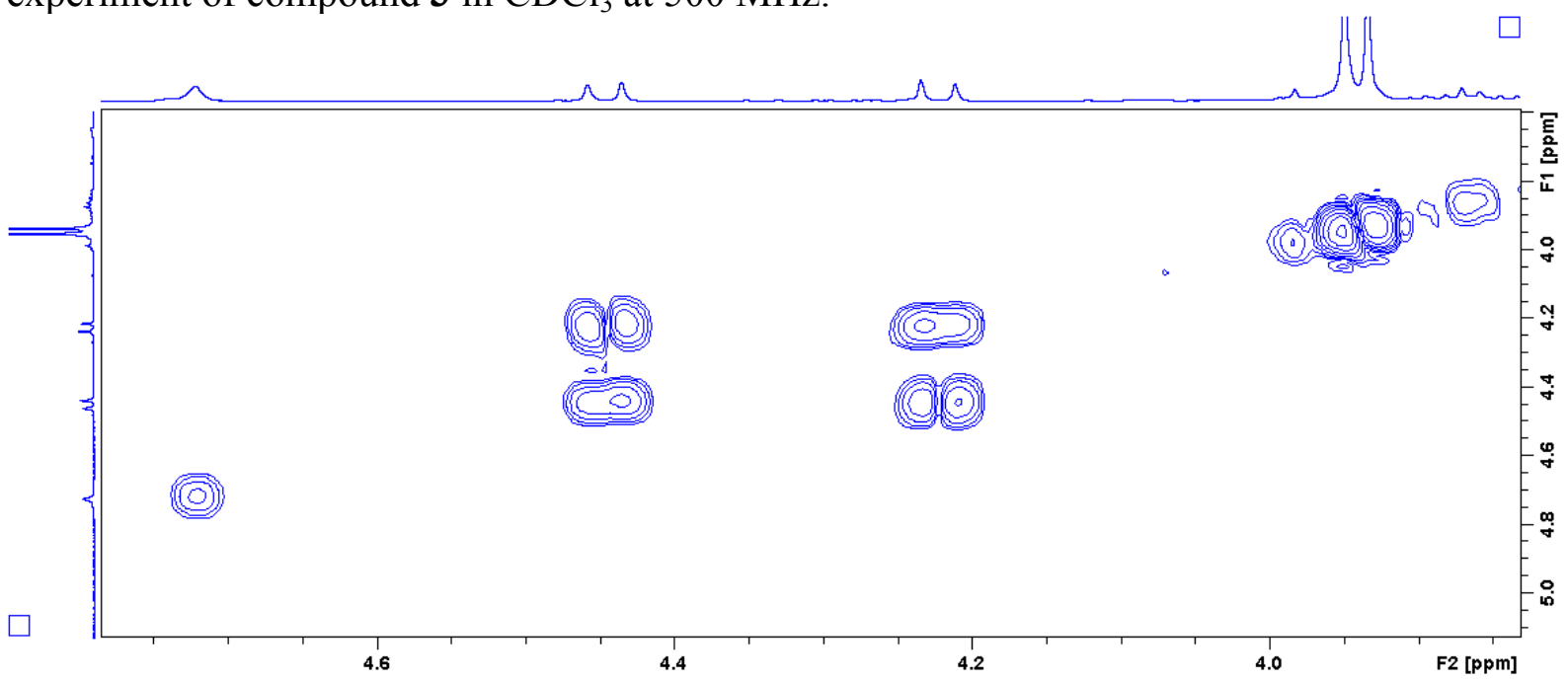

Figure S42. Enlargement $(\delta 3.32-2.49)$ of ${ }^{1} \mathrm{H}-{ }^{1} \mathrm{H}$ COSY correlation map from ${ }^{1} \mathrm{H}$ NMR experiment of compound $\mathbf{3}$ in $\mathrm{CDCl}_{3}$ at $500 \mathrm{MHz}$.

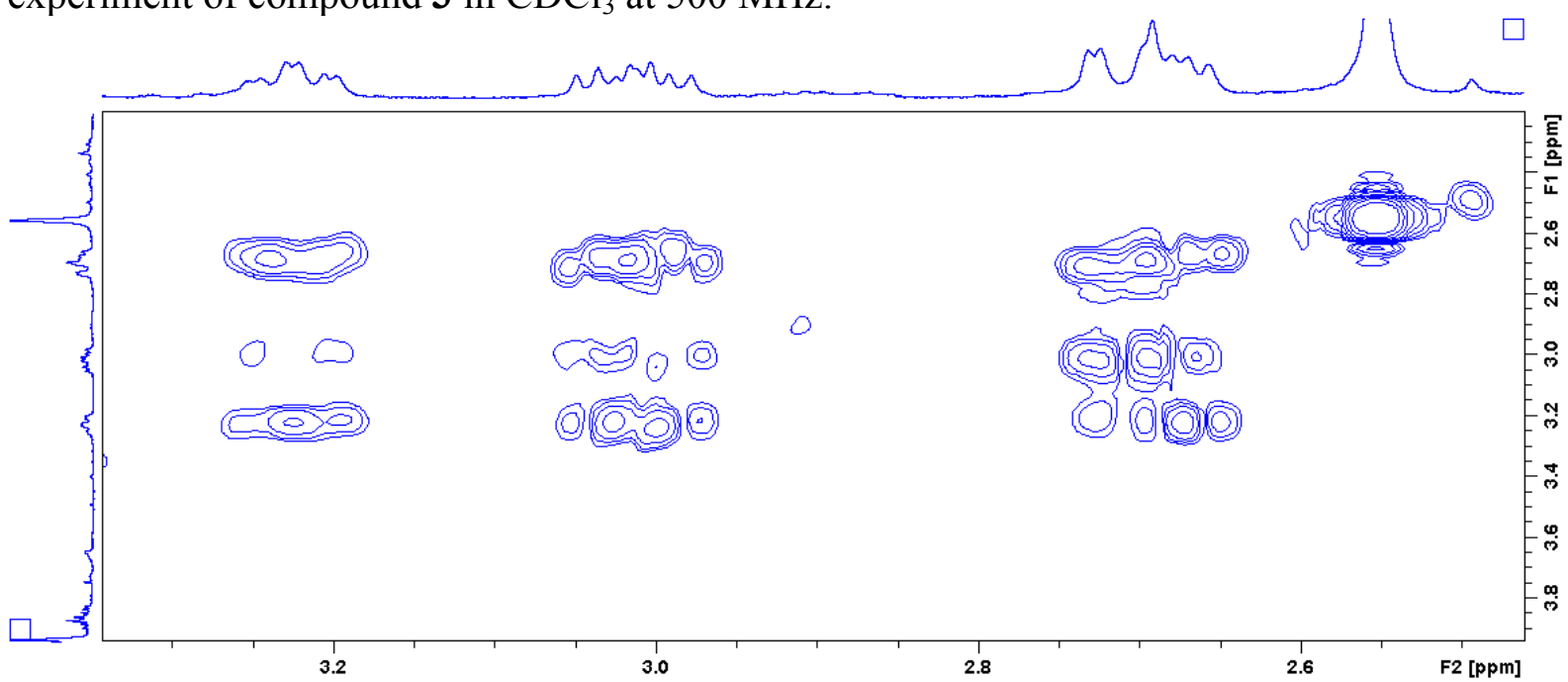


Figure S43. ${ }^{1} \mathrm{H}-{ }^{13} \mathrm{C}$ one-bond correlation map from HSQC NMR experiment of compound 3 in $\mathrm{CDCl}_{3}$ at 500 and $125 \mathrm{MHz}$.

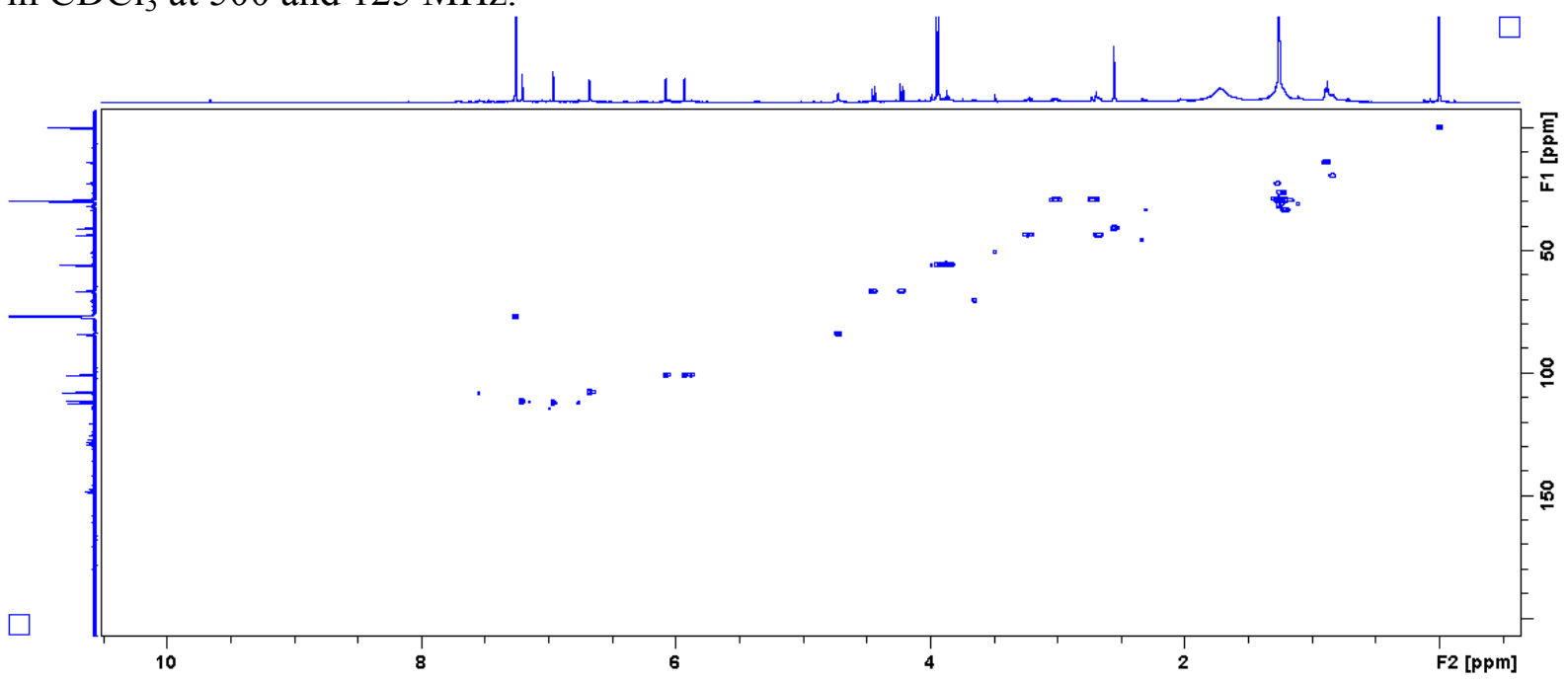

Figure S44. Enlargement $(\delta 7.20-5.93)$ of ${ }^{1} \mathrm{H}-{ }^{13} \mathrm{C}$ one-bond correlation map from HSQC NMR experiment of compound 3 in $\mathrm{CDCl}_{3}$ at 500 and $125 \mathrm{MHz}$.

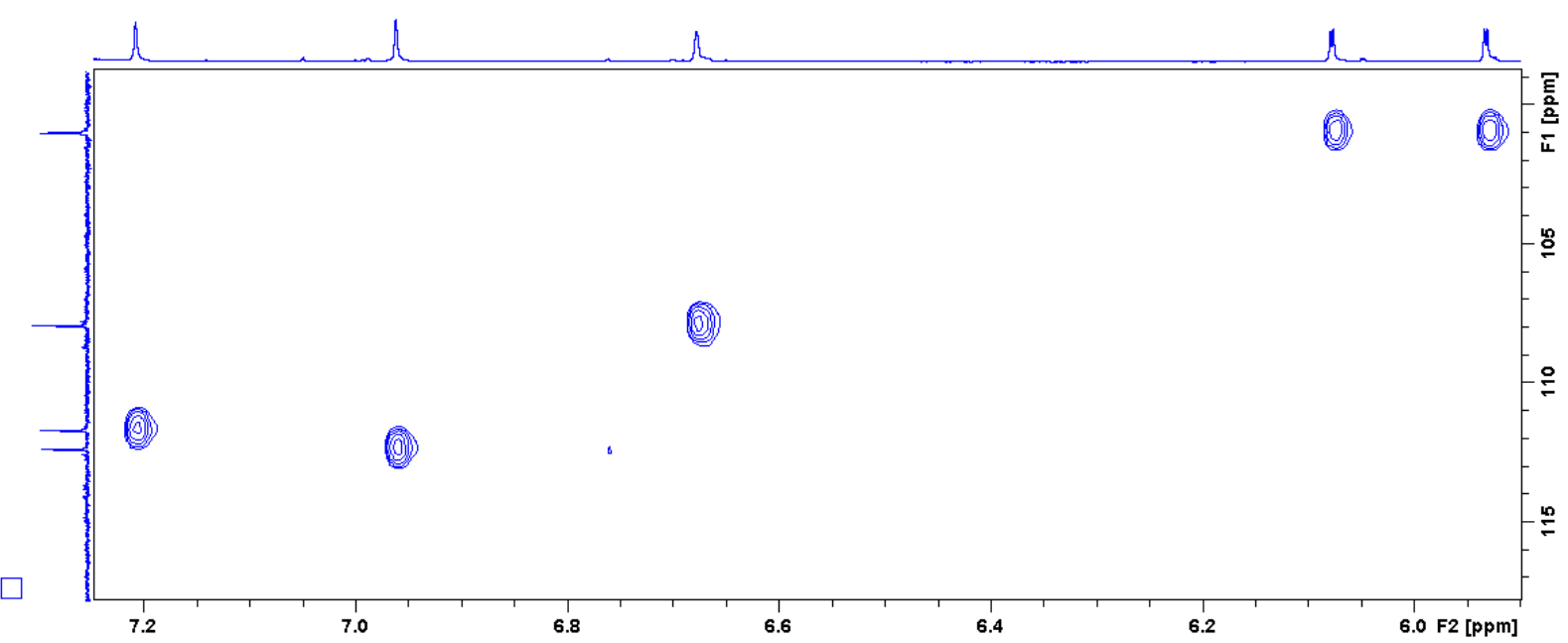

Figure S45. Enlargement $(\delta 4.90-2.55)$ of ${ }^{1} \mathrm{H}_{-}{ }^{13} \mathrm{C}$ one-bond correlation map from HSQC NMR experiment of compound 3 in $\mathrm{CDCl}_{3}$ at 500 and $125 \mathrm{MHz}$.

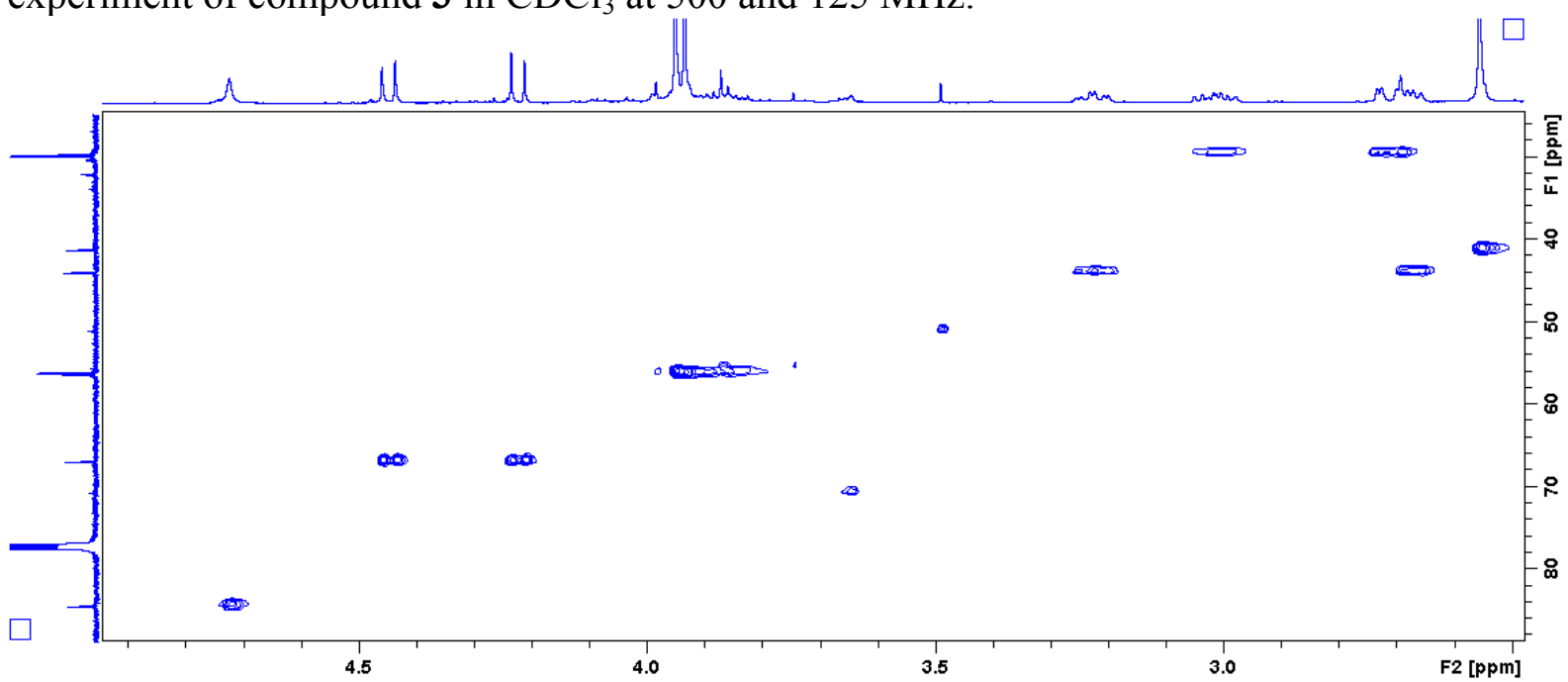


Figure S46. ${ }^{1} \mathrm{H}-{ }^{13} \mathrm{C}$ long-range correlation map from HMBC NMR experiment of compound 3 in $\mathrm{CDCl}_{3}$ at 500 and $125 \mathrm{MHz}$.

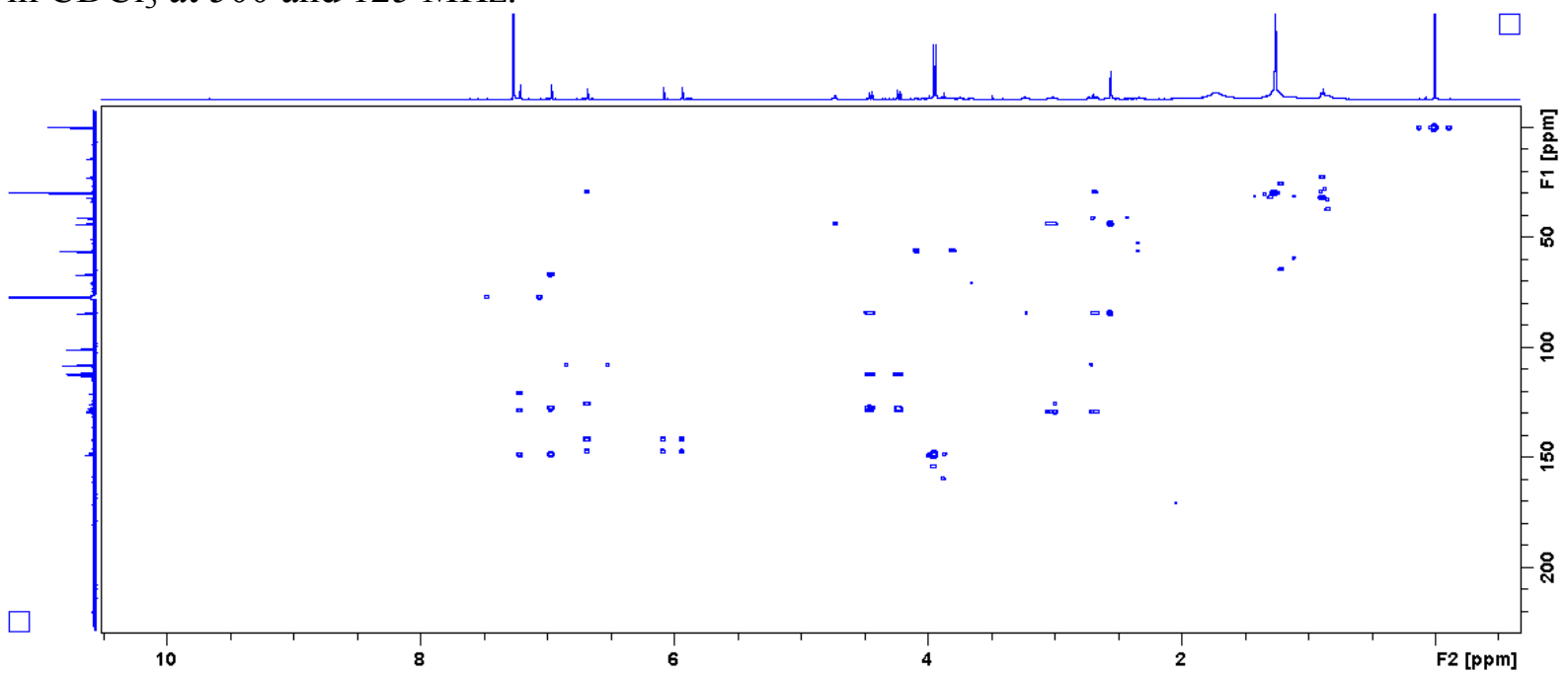

Figure S47. Enlargement $\left(\delta\right.$ 7.60-5.93) of ${ }^{1} \mathrm{H}-{ }^{13} \mathrm{C}$ long-range correlation map from HMBC NMR experiment of compound 3 in $\mathrm{CDCl}_{3}$ at 500 and $125 \mathrm{MHz}$.

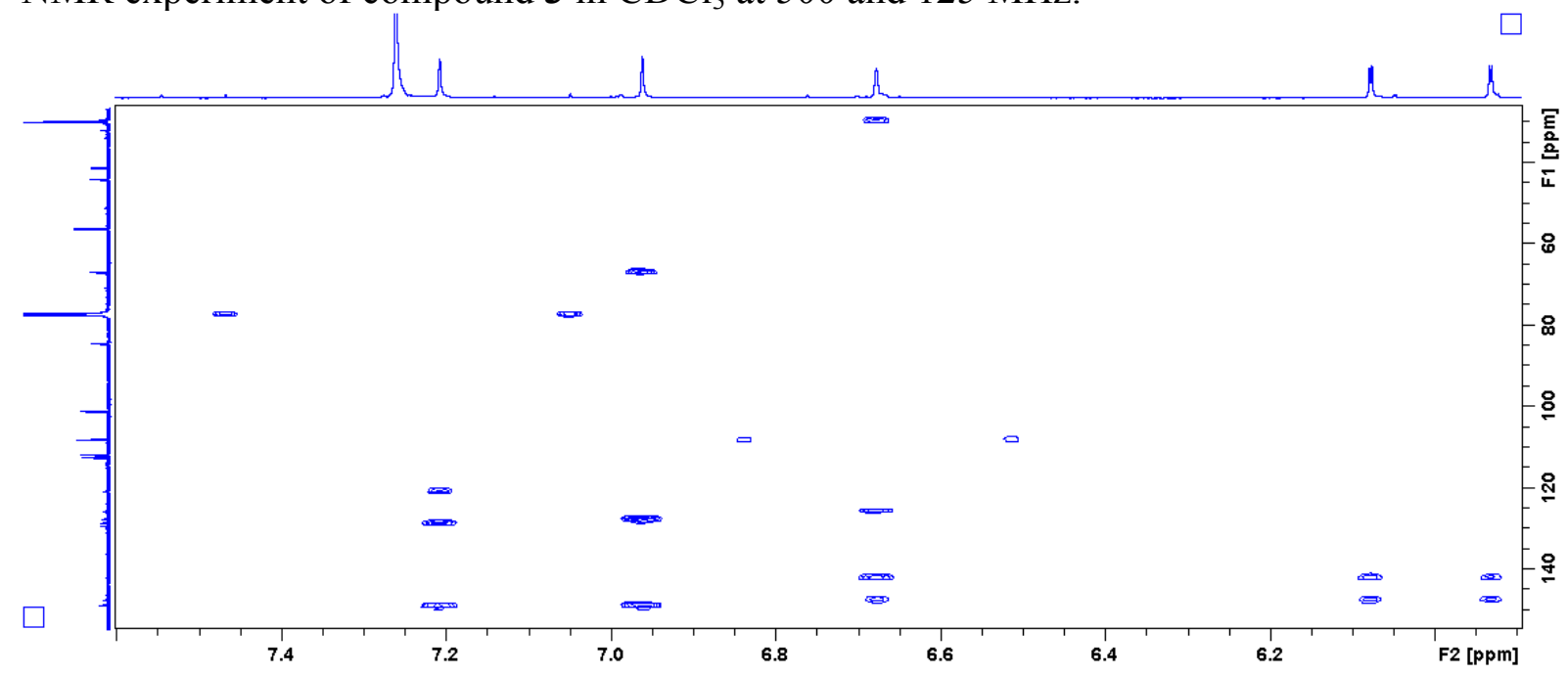

Figure S48. Enlargement $(\delta 4.72-2.55)$ of ${ }^{1} \mathrm{H}-{ }^{13} \mathrm{C}$ long-range correlation map from HMBC NMR experiment of compound 3 in $\mathrm{CDCl}_{3}$ at 500 and $125 \mathrm{MHz}$.

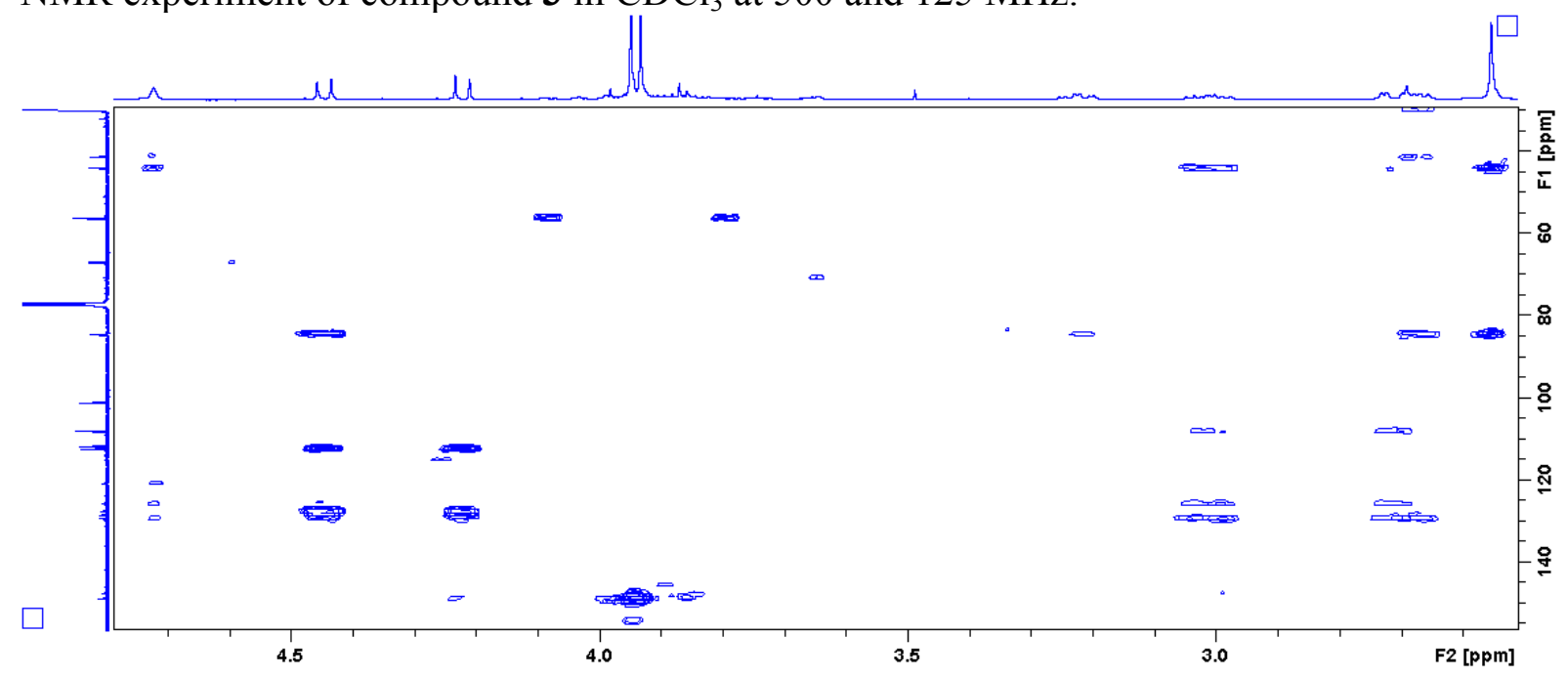


Figure S49. Enlargement of $\mathrm{NOE}(\delta 7.20)$ experiments of compound 3 in $\mathrm{CDCl}_{3}$ at $500 \mathrm{MHz}$.

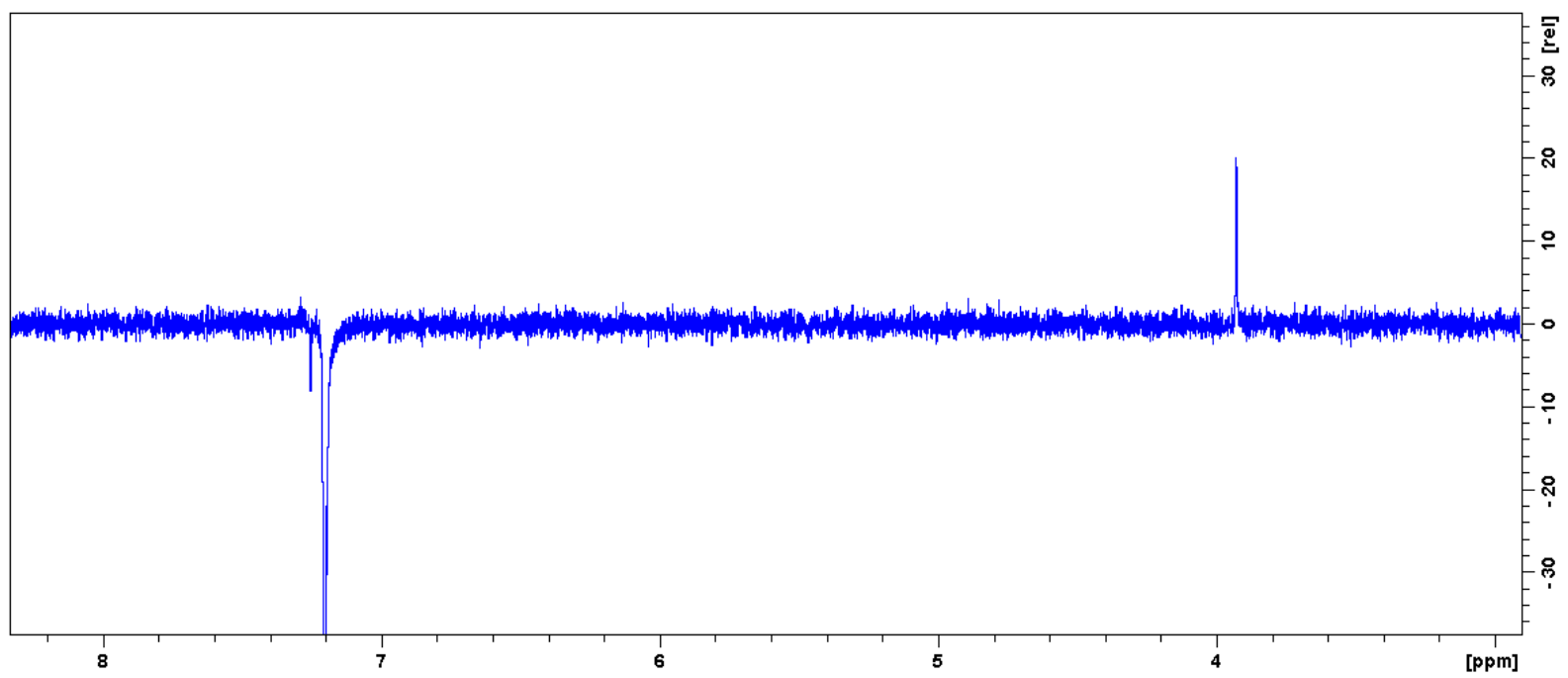

Figure S50. Enlargement of $\mathrm{NOE}(\delta 6.95)$ experiments of compound 3 in $\mathrm{CDCl}_{3}$ at $500 \mathrm{MHz}$.

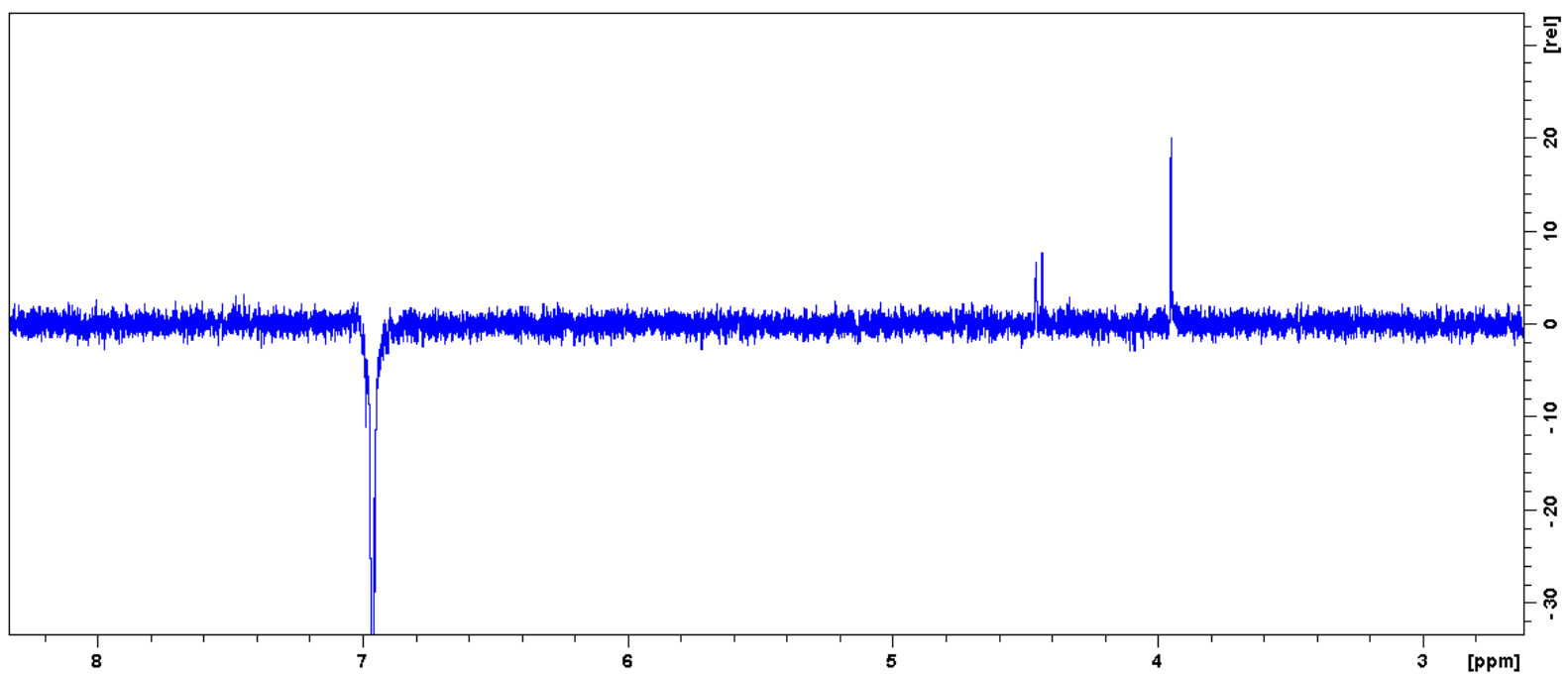

Figure S51. Enlargement of $\mathrm{NOE}\left(\delta\right.$ 6.67) experiments of compound 3 in $\mathrm{CDCl}_{3}$ at $500 \mathrm{MHz}$.

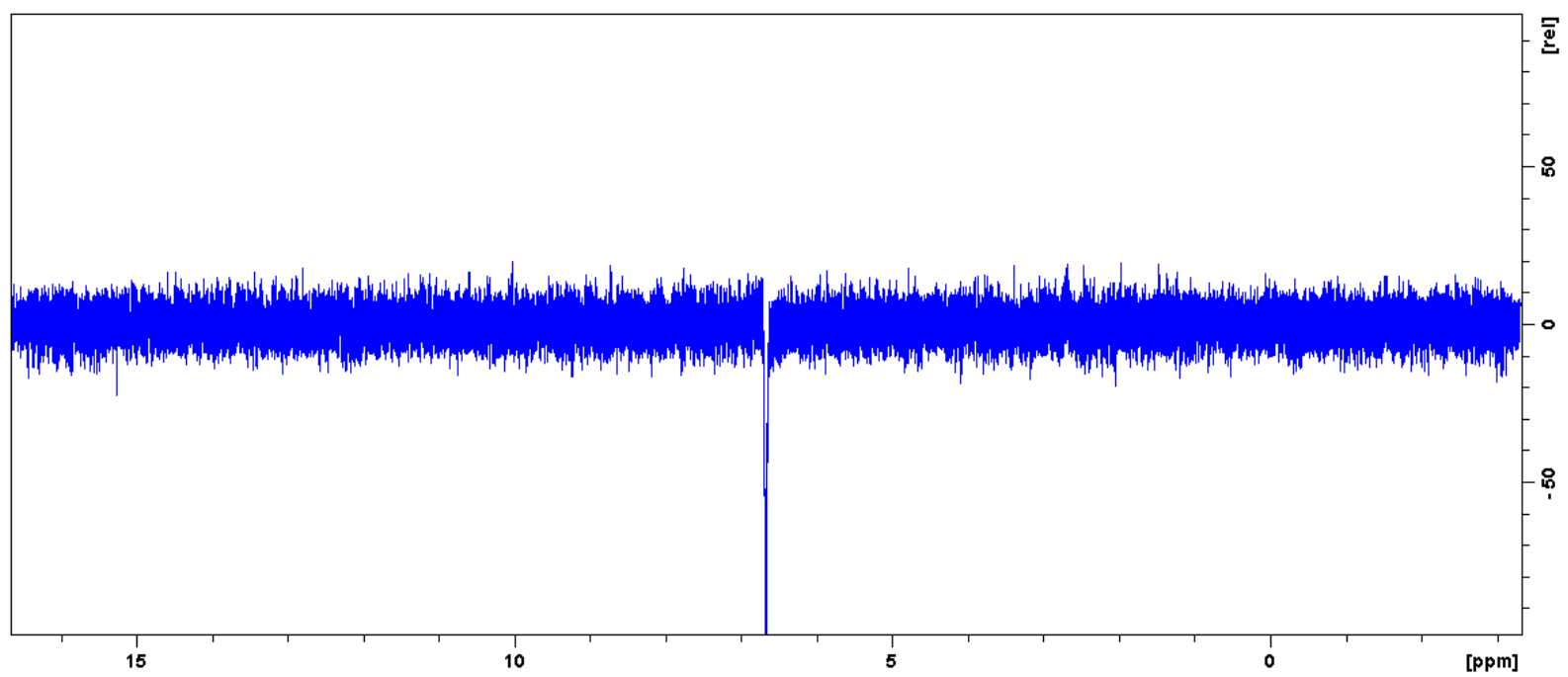


Figure S52. Enlargement of $\mathrm{NOE}(\delta 4.43)$ experiments of compound 3 in $\mathrm{CDCl}_{3}$ at $500 \mathrm{MHz}$.

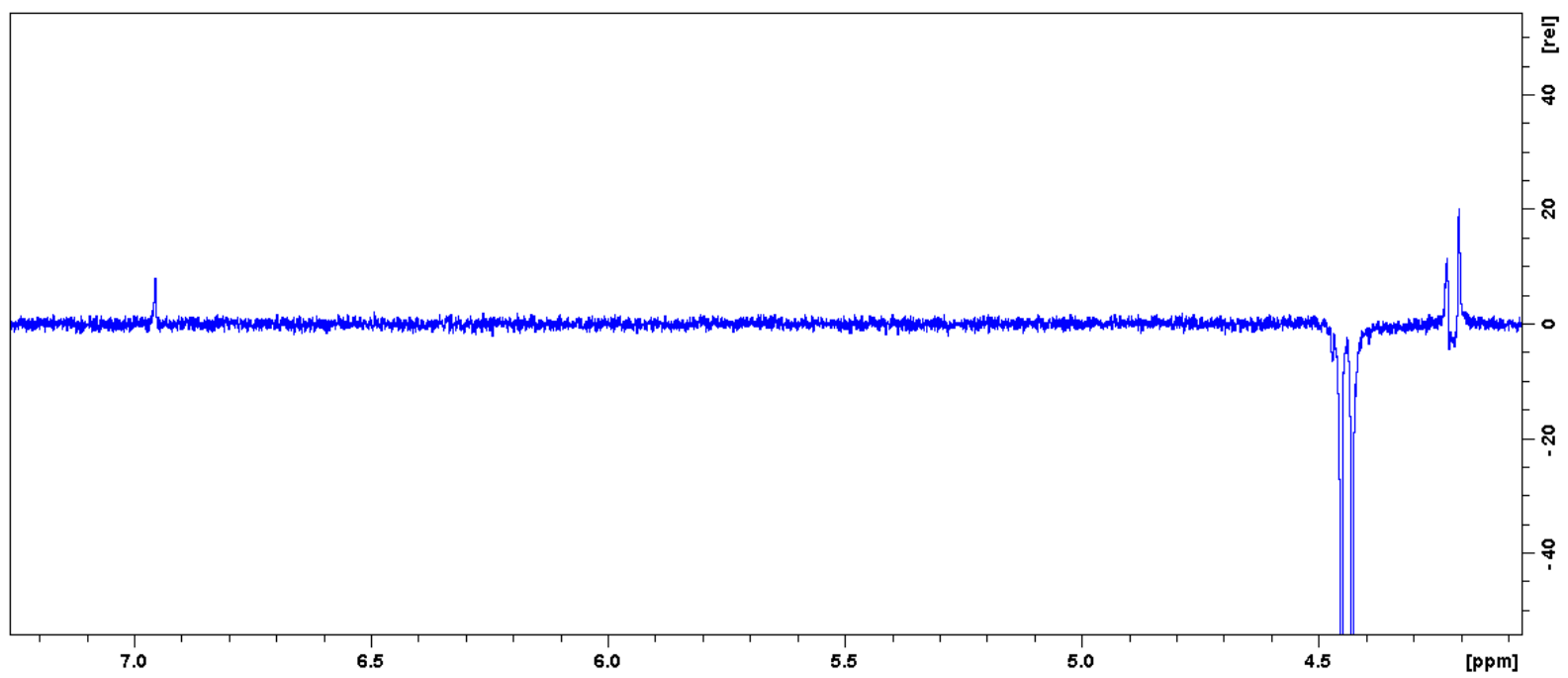

Figure S53. Enlargement of $\mathrm{NOE}(\delta 4.22)$ experiments of compound 3 in $\mathrm{CDCl}_{3}$ at $500 \mathrm{MHz}$.

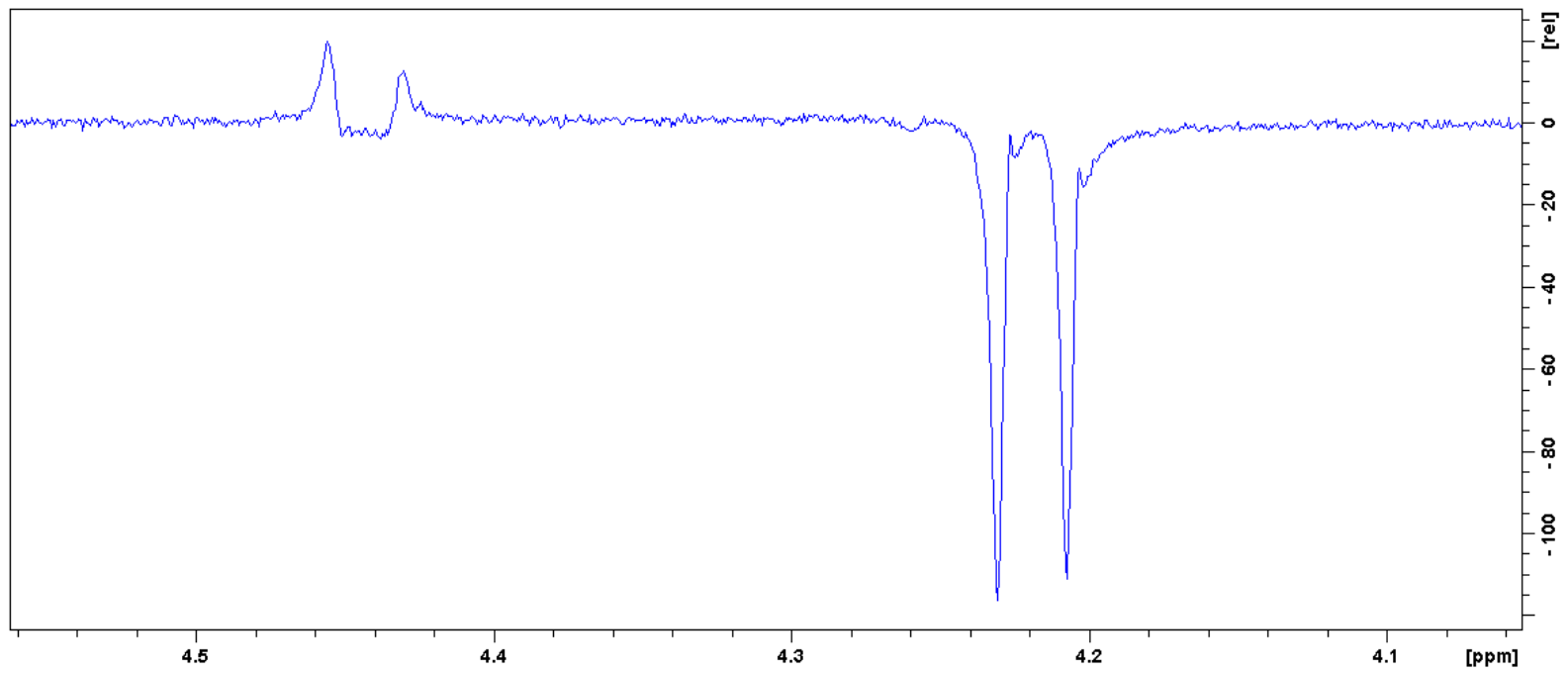

Figure S54. Enlargement of $\mathrm{NOE}(\delta 3.22)$ experiments of compound 3 in $\mathrm{CDCl}_{3}$ at $500 \mathrm{MHz}$.

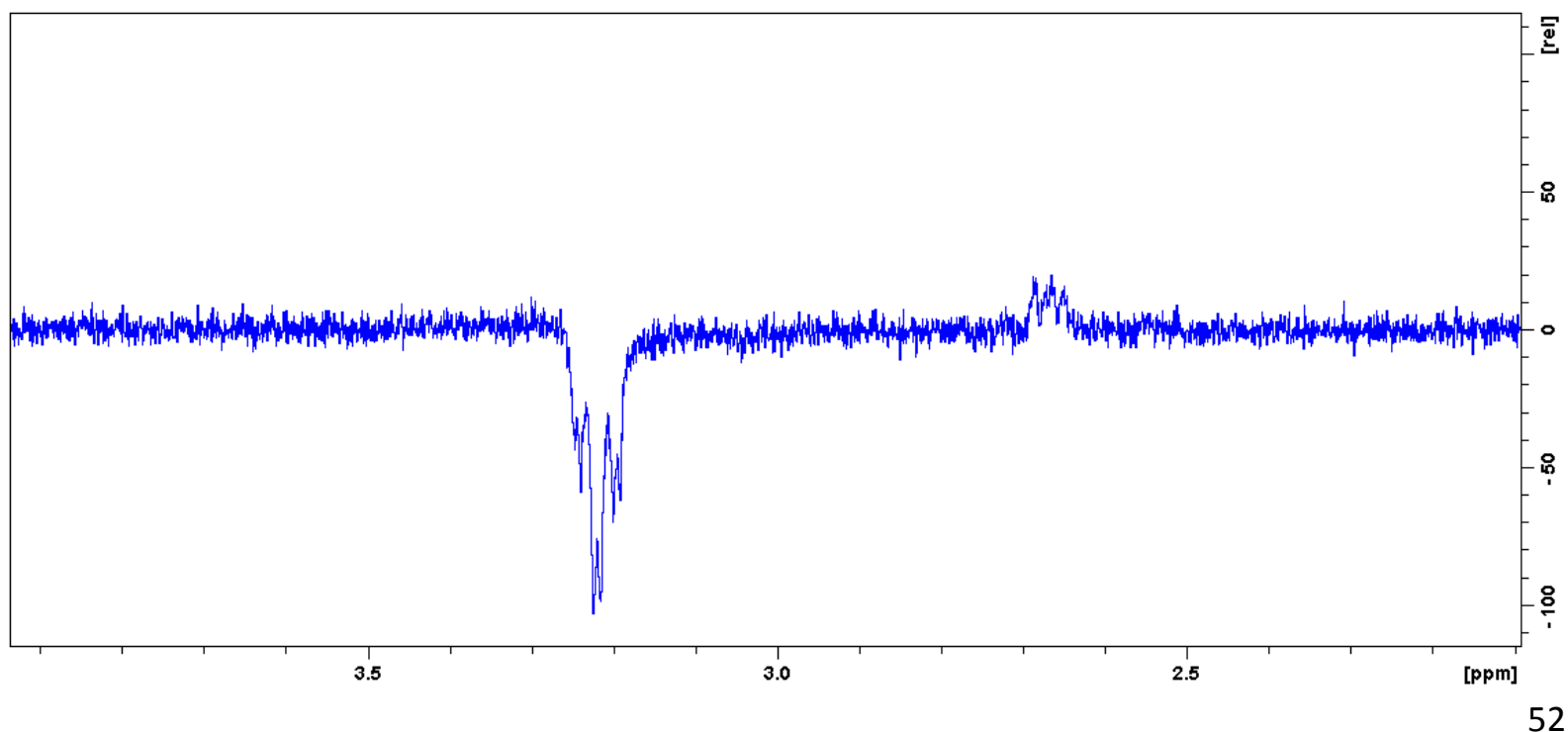


Figure S55. Enlargement of $\mathrm{NOE}(\delta 3.00)$ experiments of compound 3 in $\mathrm{CDCl}_{3}$ at $500 \mathrm{MHz}$.

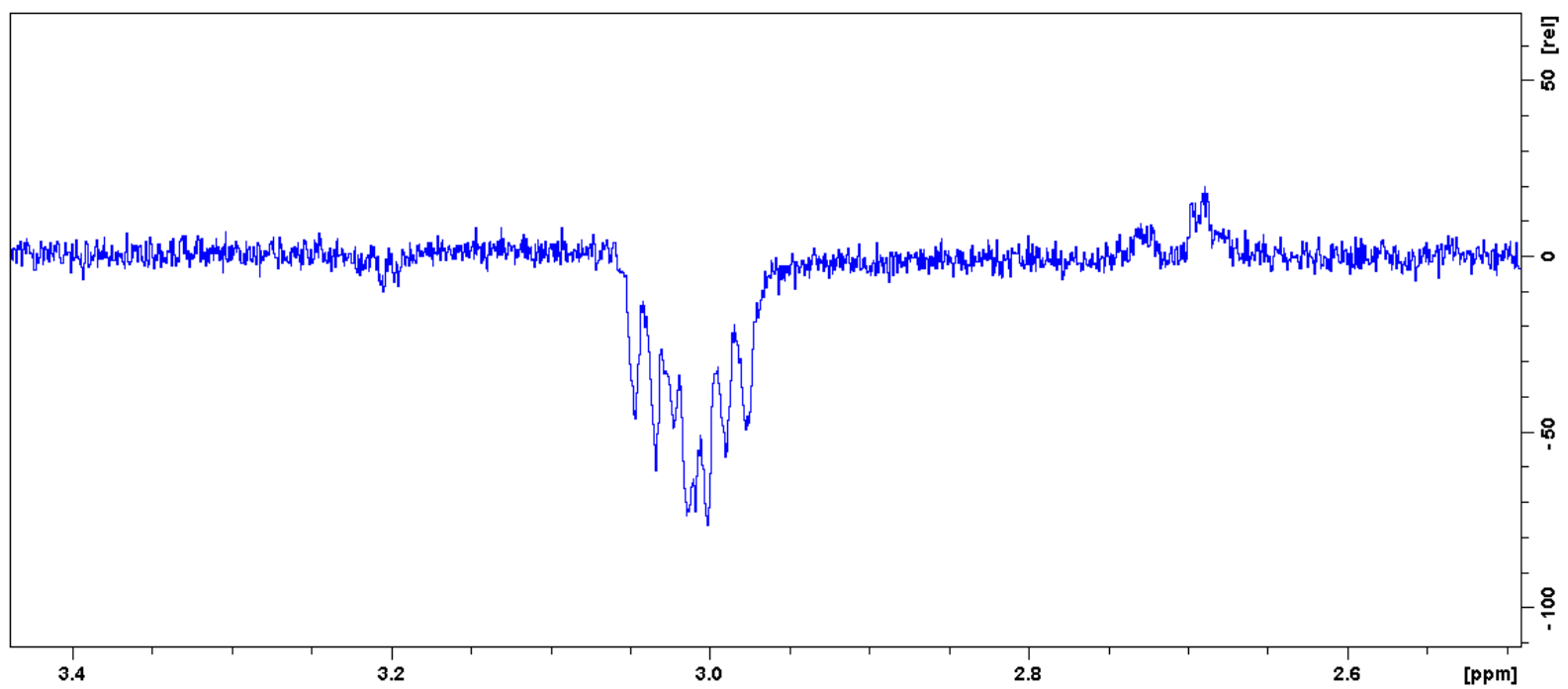

Figure S56. Enlargement of NOE ( $\delta$ 2.55) experiments of compound 3 in $\mathrm{CDCl}_{3}$ at $500 \mathrm{MHz}$.

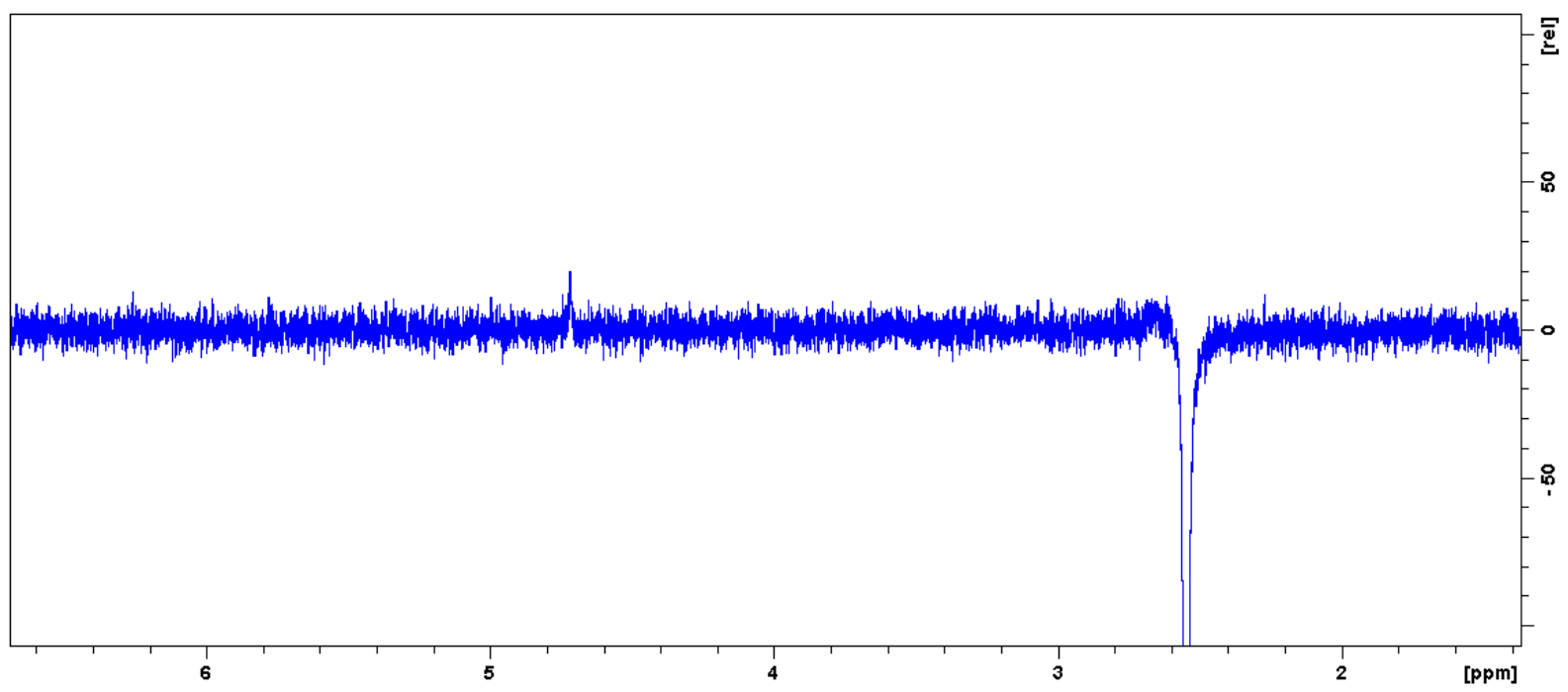


Figure S57. HRESIMS spectrum of compound 3.

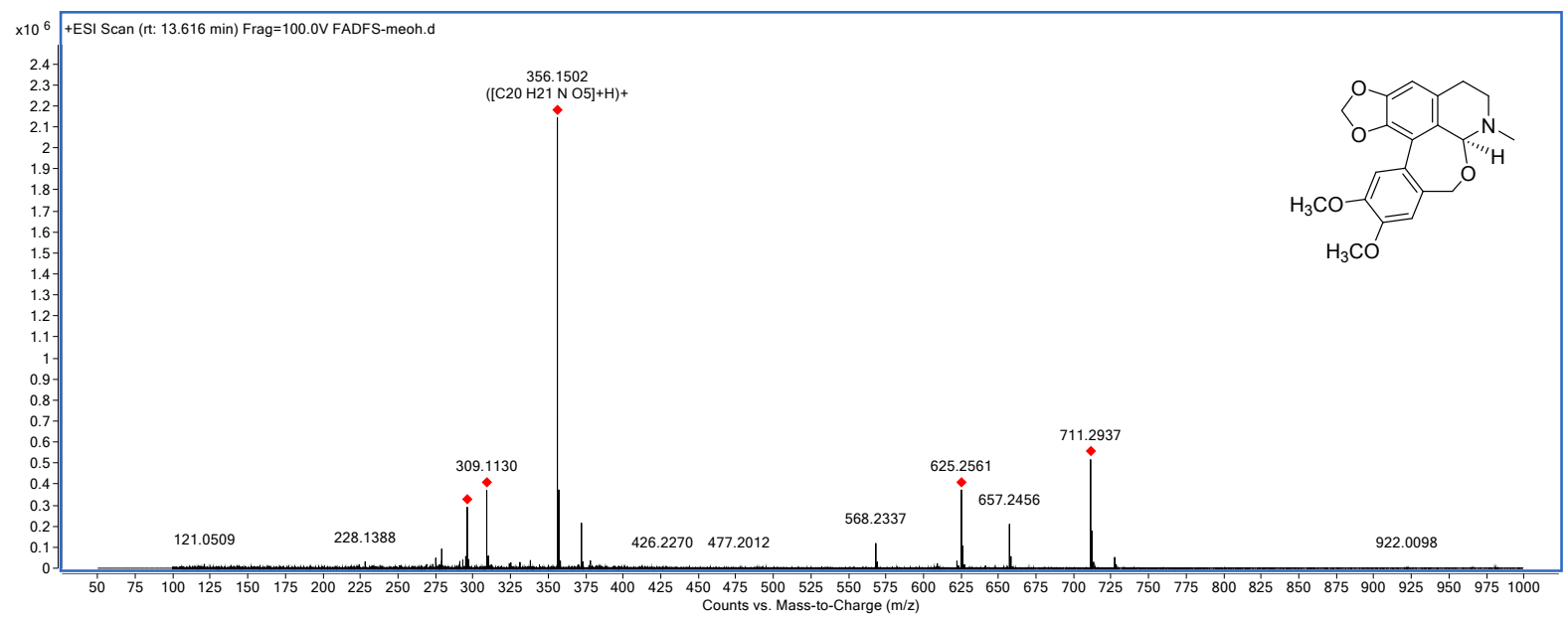

54 
Figure S58. Extracted ion chromatogram, MS and MS/MS spectra of compound 3.
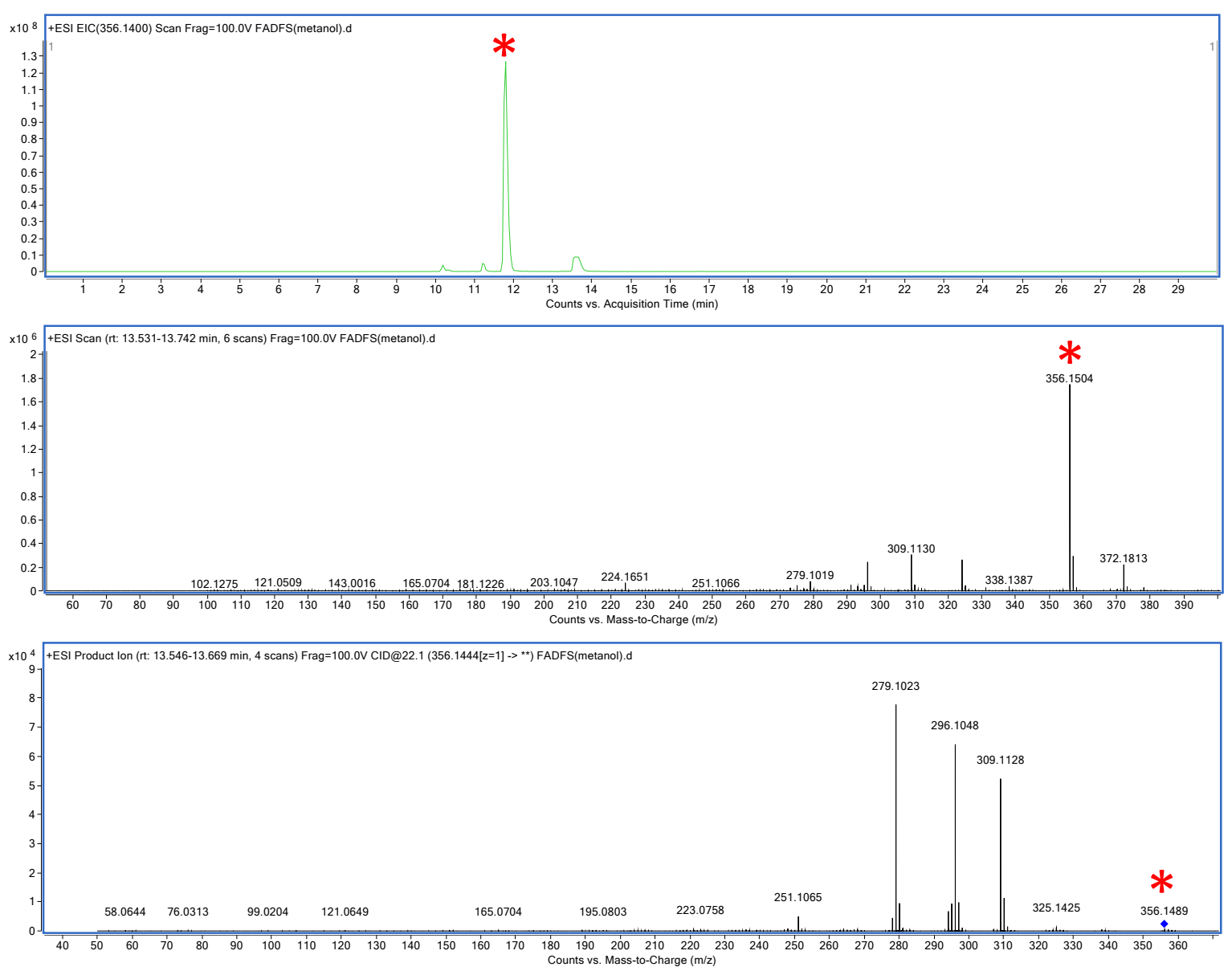
Figure S59. ${ }^{1} \mathrm{H}$ NMR spectrum of compound 4 in $\mathrm{CDCl}_{3}$ at $500 \mathrm{MHz}$. 


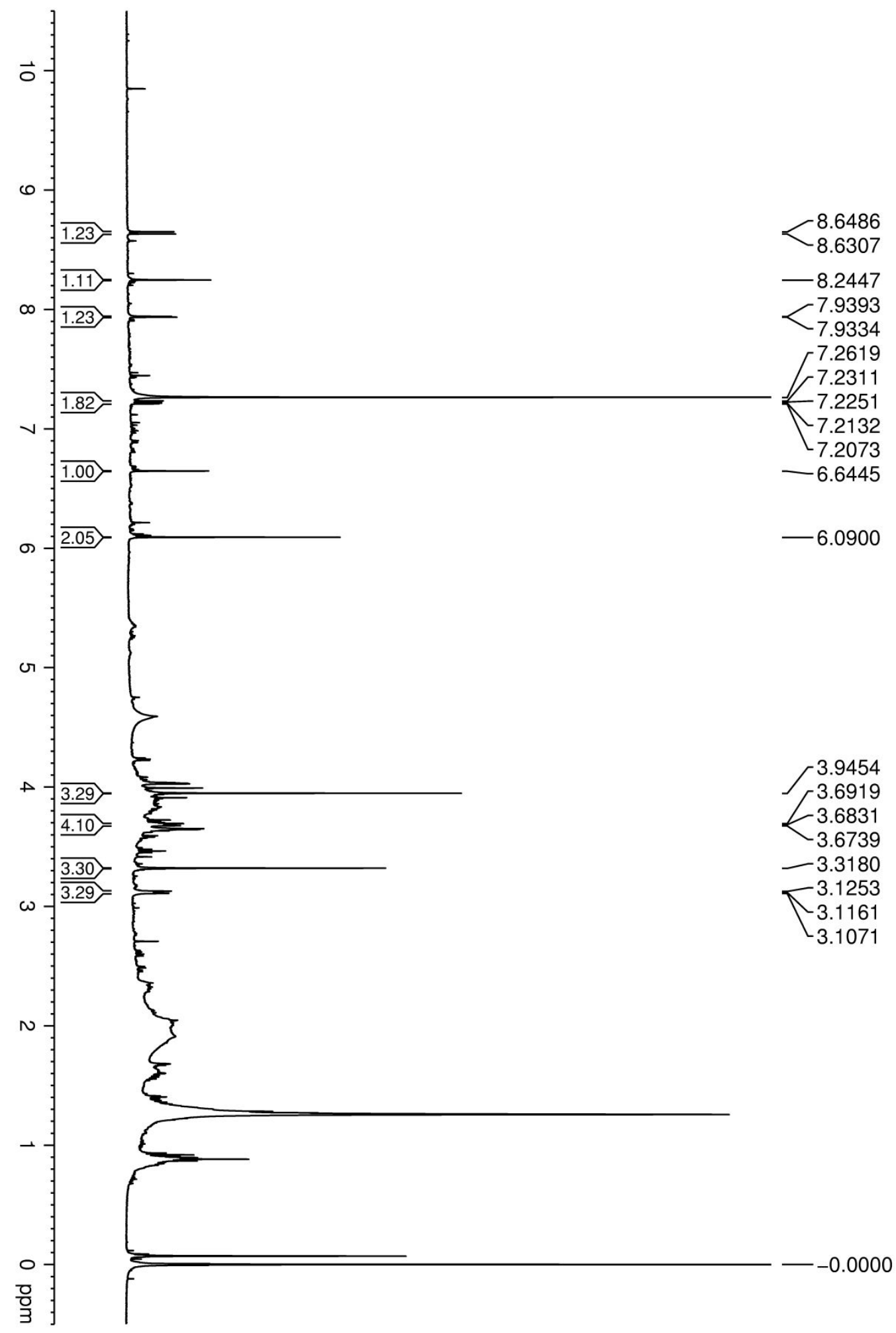

Figure S60. Enlargement ( $\delta$ 8.64-6.09) of ${ }^{1} \mathrm{H}$ NMR spectrum of compound 4 in $\mathrm{CDCl}_{3}$ at 500 $\mathrm{MHz}$. 


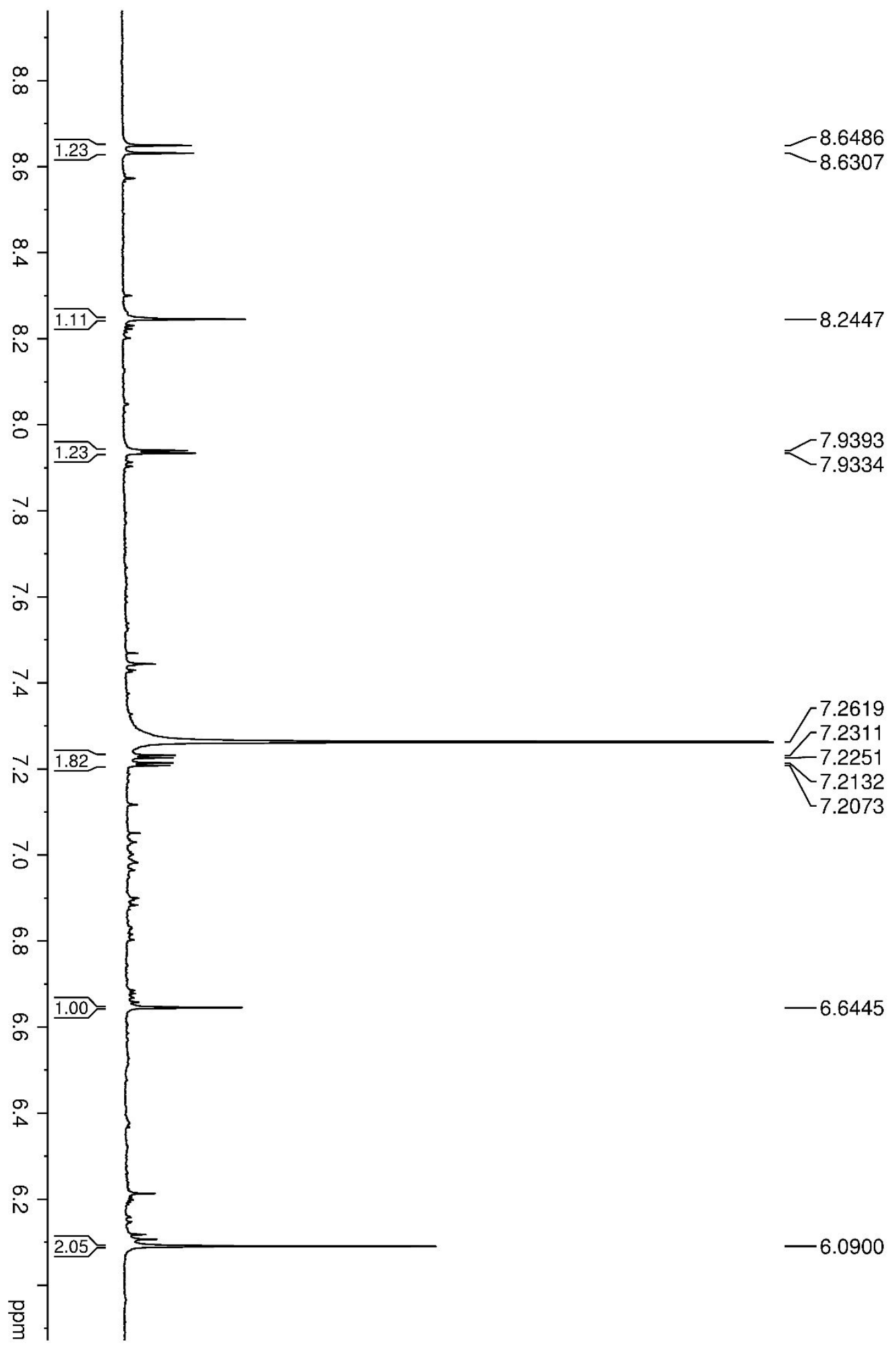

Figure S61. Enlargement ( $\delta$ 8.64-7.93) of ${ }^{1} \mathrm{H}$ NMR spectrum of compound 4 in $\mathrm{CDCl}_{3}$ at 500 $\mathrm{MHz}$. 

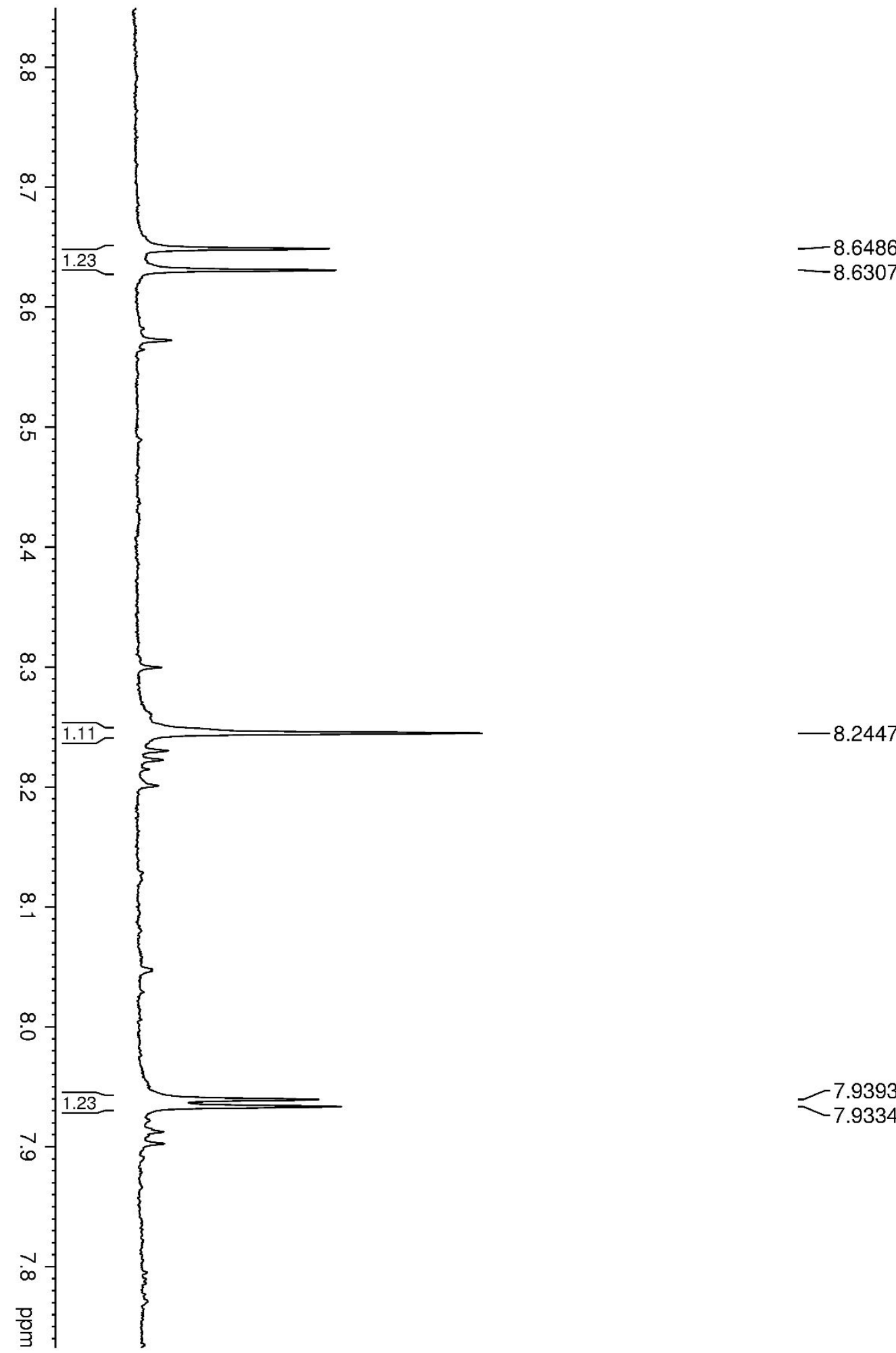

Figure S62. Enlargement ( $\delta$ 7.26-6.09) of ${ }^{1} \mathrm{H}$ NMR spectrum of compound 4 in $\mathrm{CDCl}_{3}$ at 500 $\mathrm{MHz}$. 


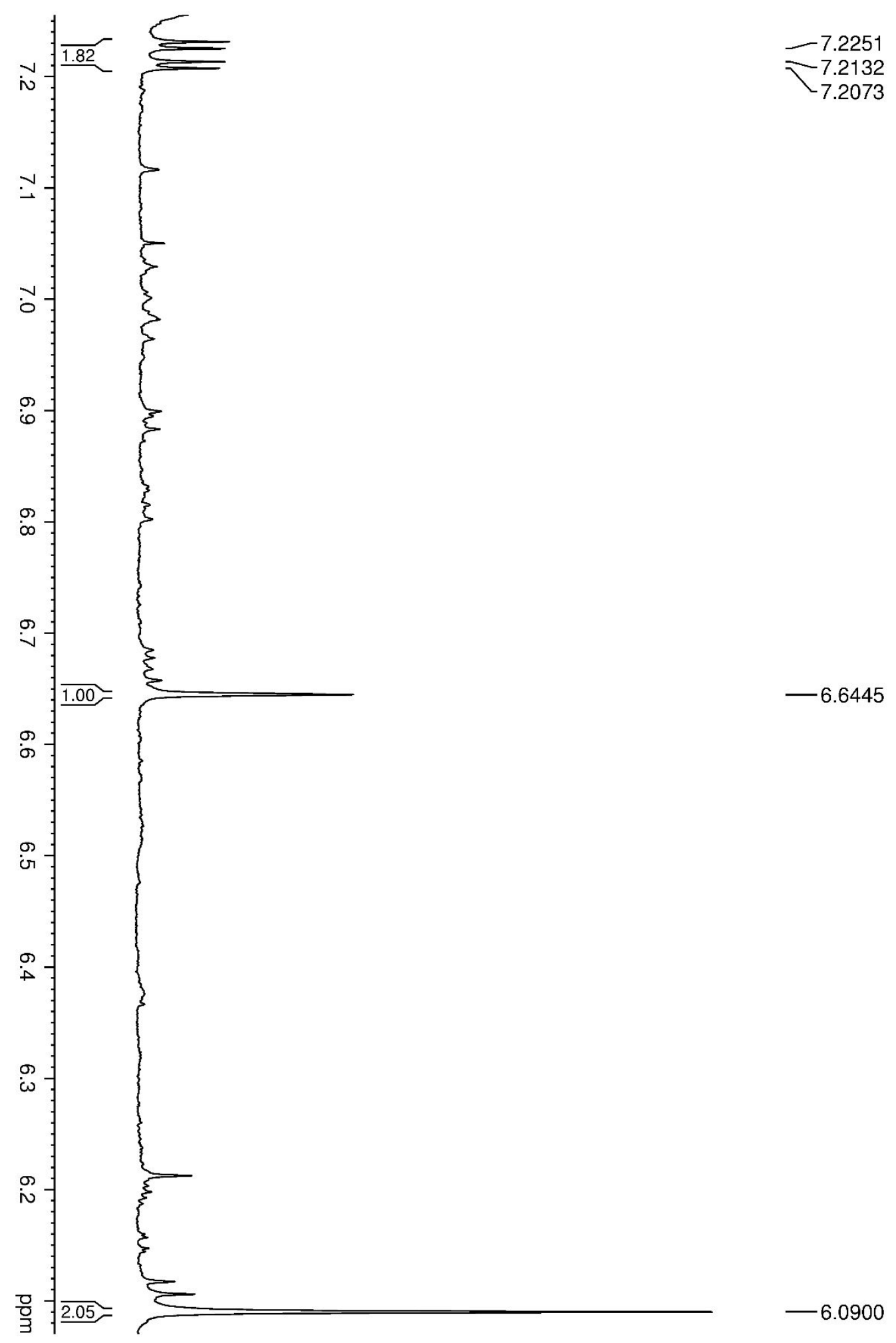

Figure S63. Enlargement ( $\delta 3.94-3.10)$ of ${ }^{1} \mathrm{H}$ NMR spectrum of compound 4 in $\mathrm{CDCl}_{3}$ at 500 $\mathrm{MHz}$. 


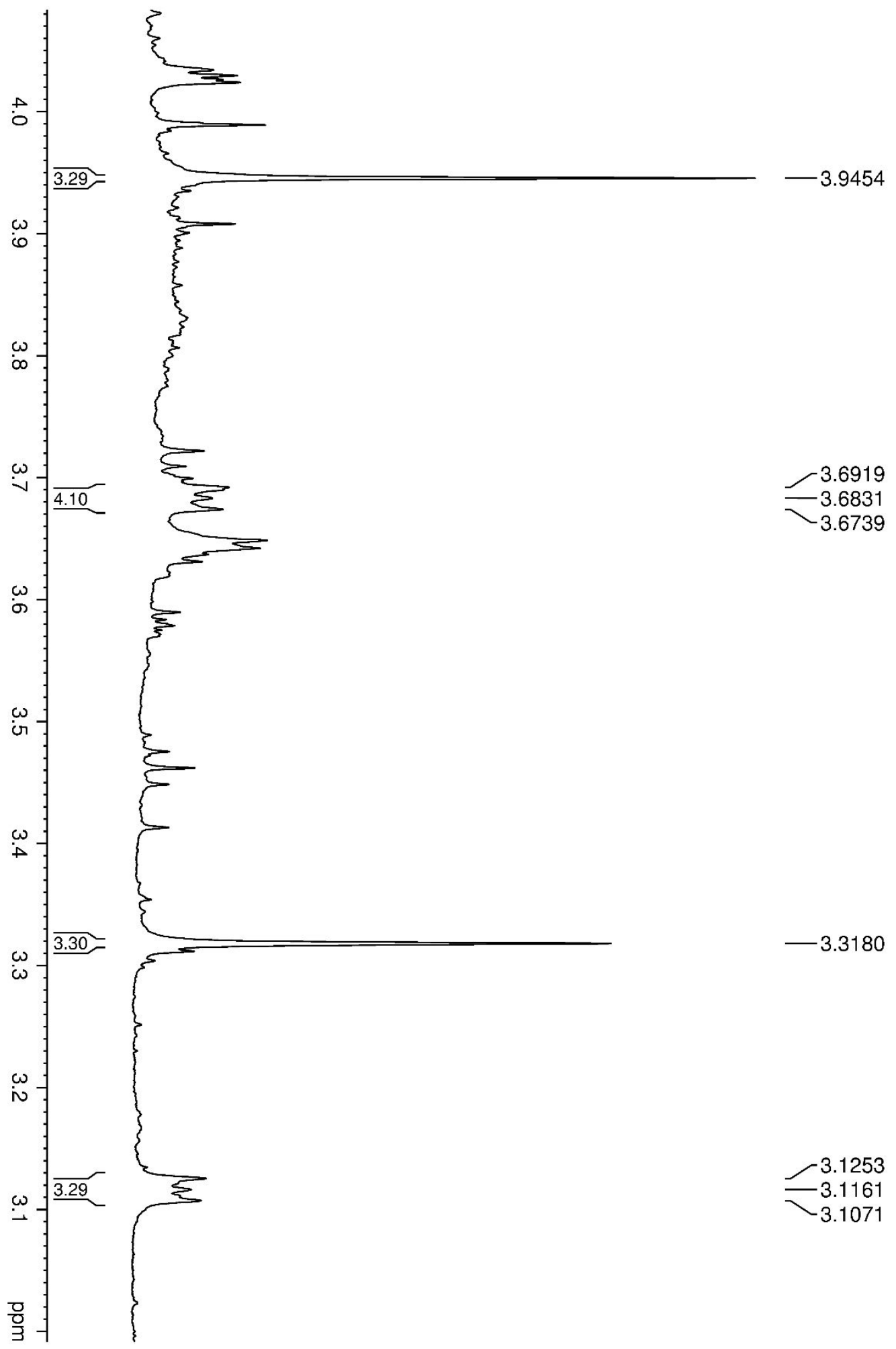

Figure S64. Enlargement ( $\delta 3.69-3.10)$ of ${ }^{1} \mathrm{H}$ NMR spectrum of compound 4 in $\mathrm{CDCl}_{3}$ at 500 $\mathrm{MHz}$. 


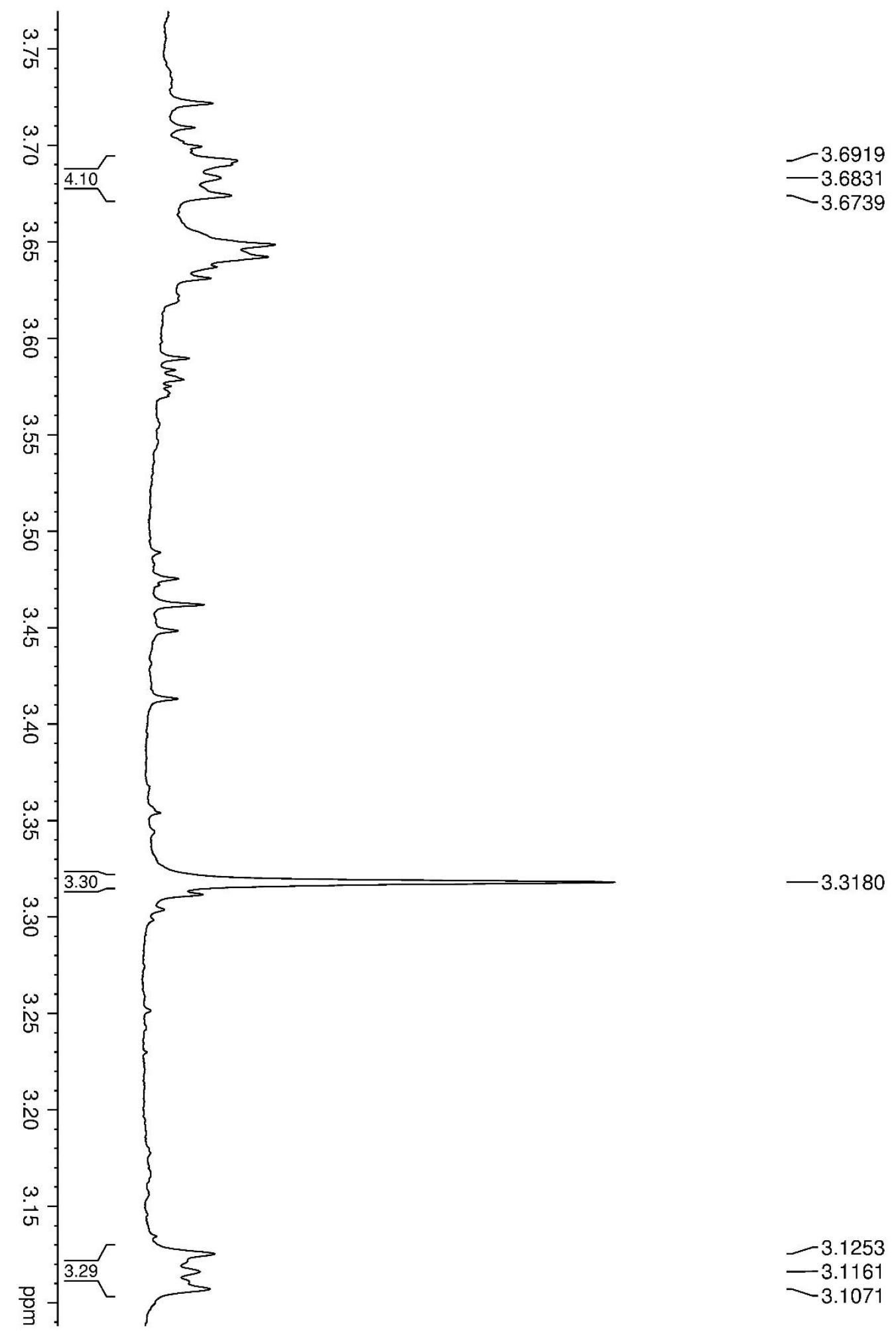

Figure S65. ${ }^{13} \mathrm{C}$ NMR spectrum of compound 4 in $\mathrm{CDCl}_{3}$ at $125 \mathrm{MHz}$. 


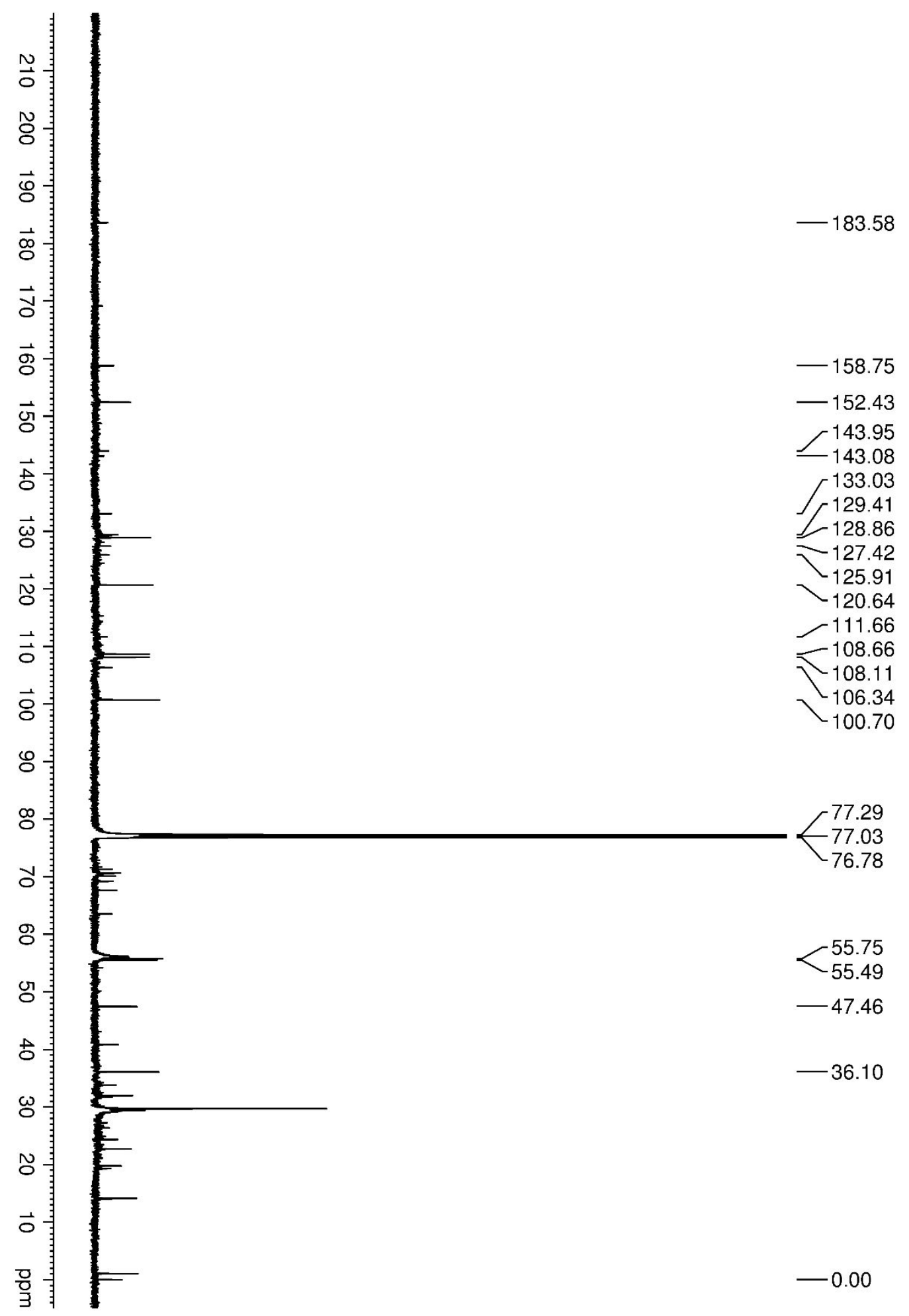


Figure S66. Enlargement ( $\delta$ 183.5-100.6) of ${ }^{13} \mathrm{C}$ NMR spectrum of compound 4 in $\mathrm{CDCl}_{3}$ at $125 \mathrm{MHz}$.

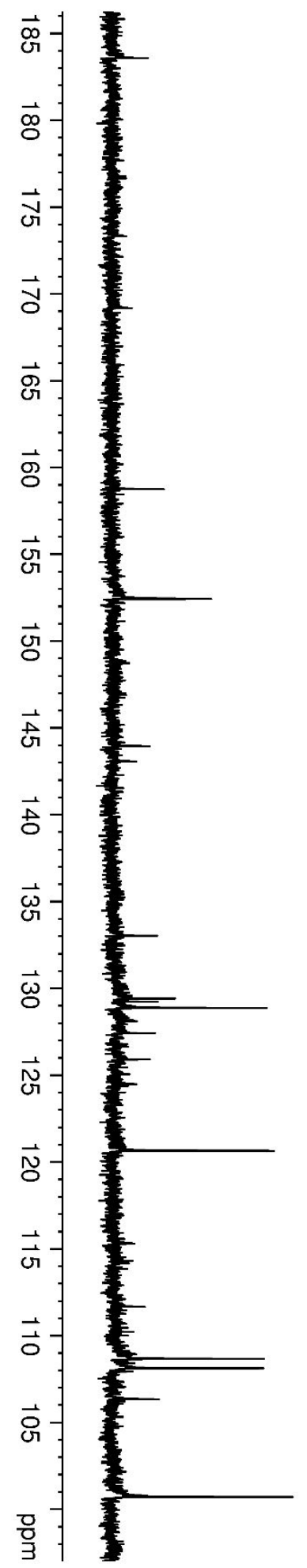


Figure S67. Enlargement ( $\delta 55.7-36.0)$ of ${ }^{13} \mathrm{C}$ NMR spectrum of compound 4 in $\mathrm{CDCl}_{3}$ at 125 $\mathrm{MHz}$.

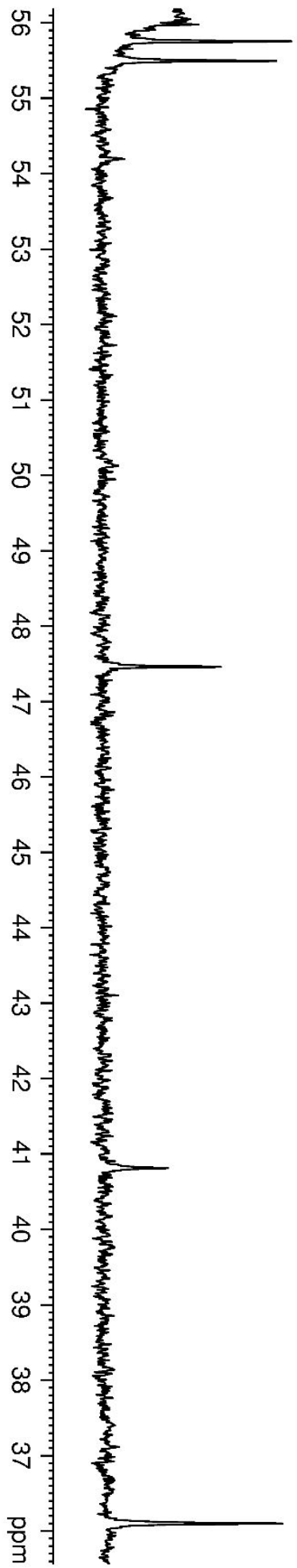

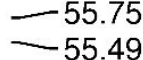

$-47.46$

$-36.10$ 
Figure S68. ${ }^{1} \mathrm{H}-{ }^{1} \mathrm{H}$ COSY correlation map from ${ }^{1} \mathrm{H}$ NMR experiment of compound 4 in $\mathrm{CDCl}_{3}$ at $500 \mathrm{MHz}$.

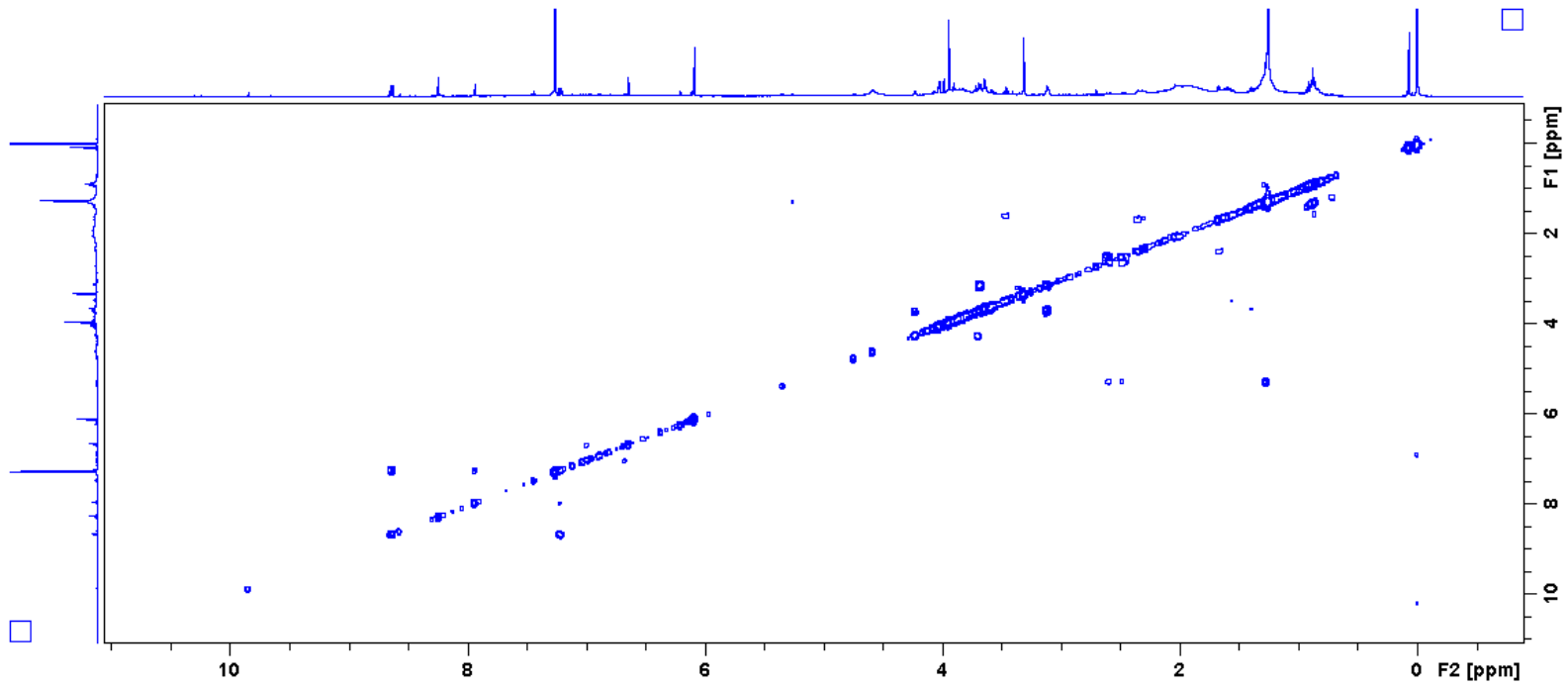

Figure S69. Enlargement $\left(\delta\right.$ 8.64-7.21) of ${ }^{1} \mathrm{H}-{ }^{1} \mathrm{H}$ COSY correlation map from ${ }^{1} \mathrm{H}$ NMR experiment of compound 4 in $\mathrm{CDCl}_{3}$ at $500 \mathrm{MHz}$.

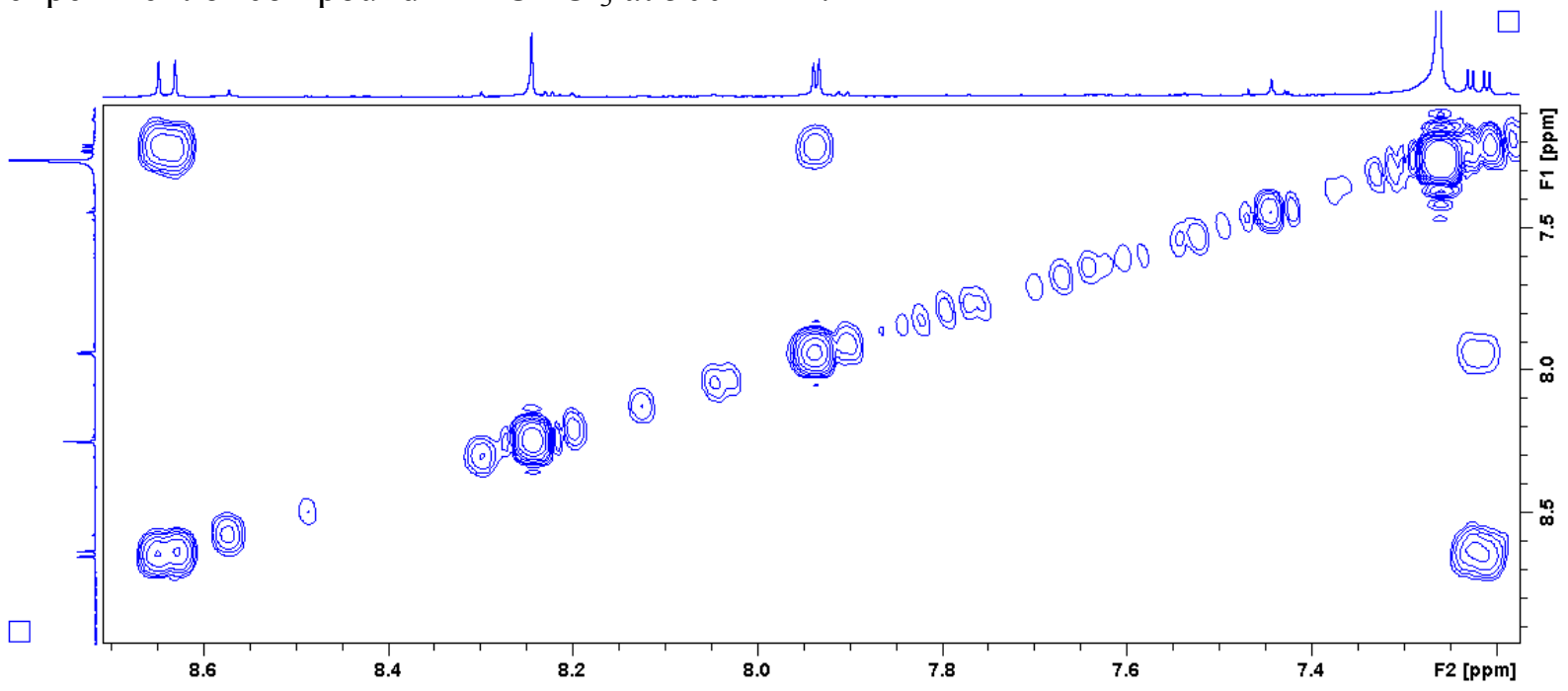

Figure S70. Enlargement $\left(\delta\right.$ 3.68-3.11) of ${ }^{1} \mathrm{H}-{ }^{1} \mathrm{H}$ COSY correlation map from ${ }^{1} \mathrm{H}$ NMR experiment of compound 4 in $\mathrm{CDCl}_{3}$ at $500 \mathrm{MHz}$.

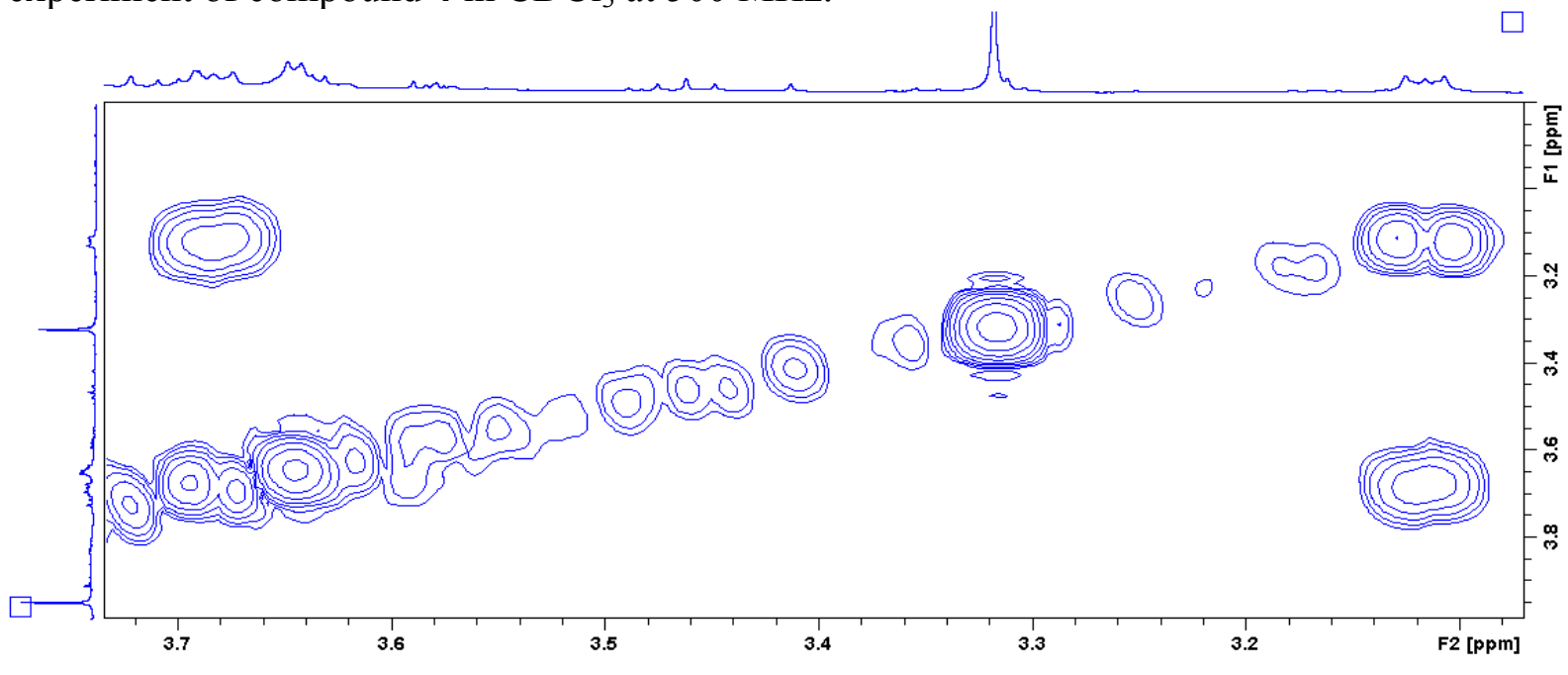


Figure S71. ${ }^{1} \mathrm{H}-{ }^{13} \mathrm{C}$ one-bond correlation map from HSQC NMR experiment of compound 4 in $\mathrm{CDCl}_{3}$ at 500 and $125 \mathrm{MHz}$.

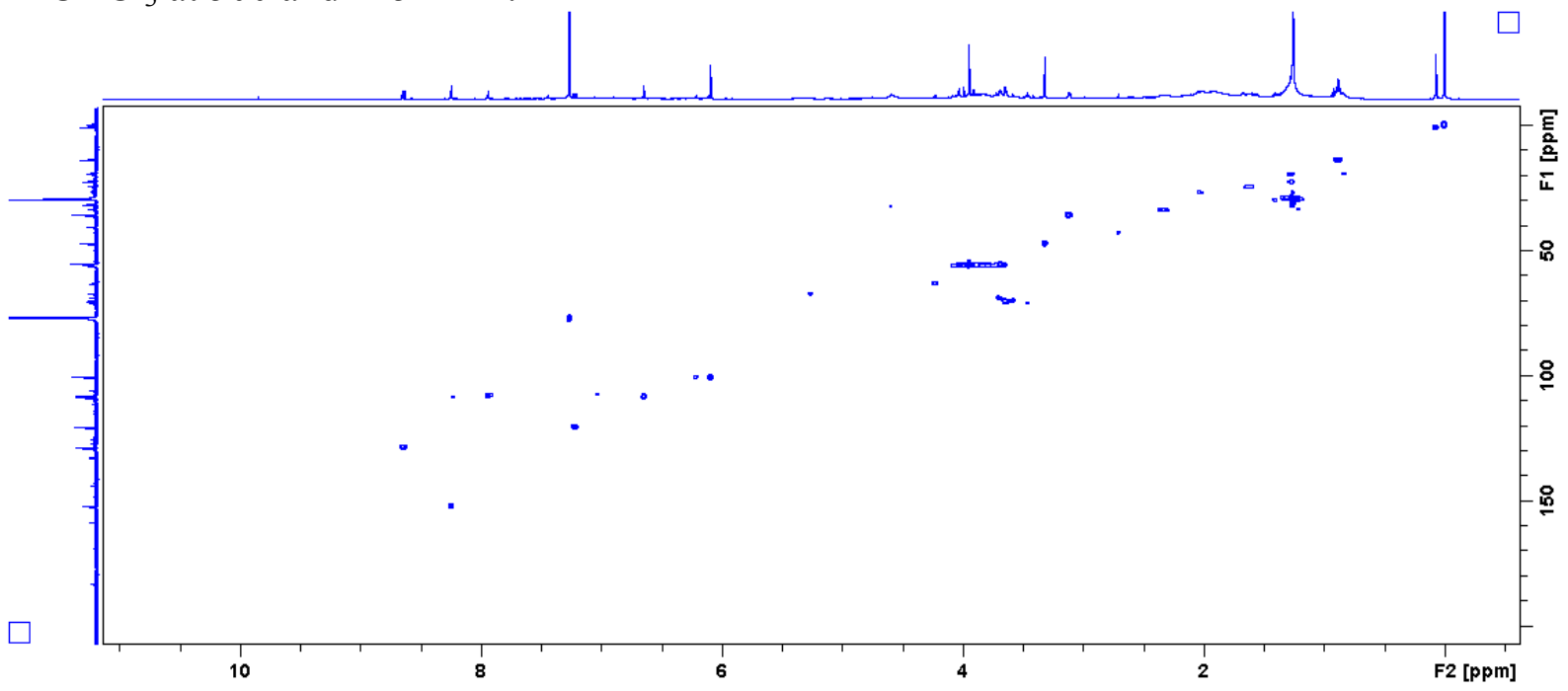

Figure S72. Enlargement $(\delta 8.64-6.08)$ of ${ }^{1} \mathrm{H}-{ }^{13} \mathrm{C}$ one-bond correlation map from HSQC NMR experiment of compound 4 in $\mathrm{CDCl}_{3}$ at 500 and $125 \mathrm{MHz}$.

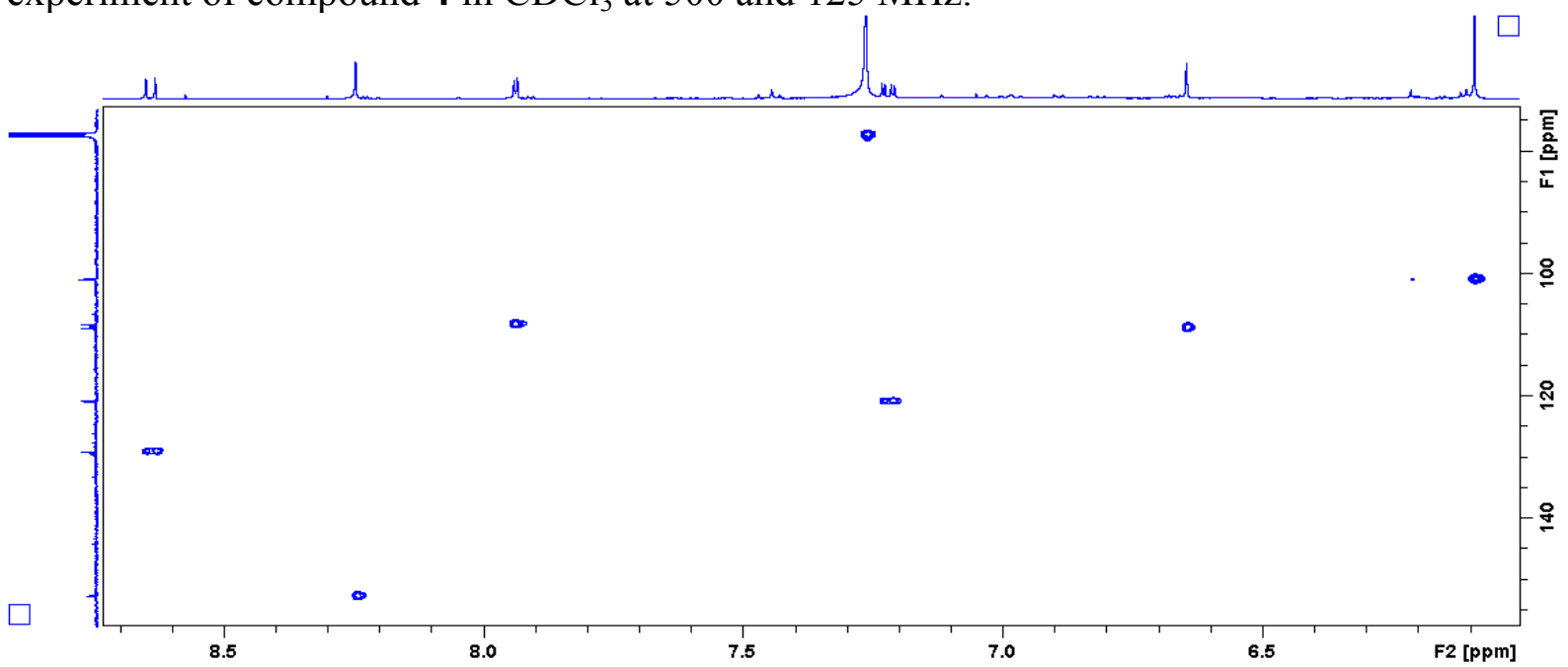

Figure S73. Enlargement $(\delta 4.05-3.11)$ of ${ }^{1} \mathrm{H}-{ }^{13} \mathrm{C}$ one-bond correlation map from HSQC NMR experiment of compound 4 in $\mathrm{CDCl}_{3}$ at 500 and $125 \mathrm{MHz}$.

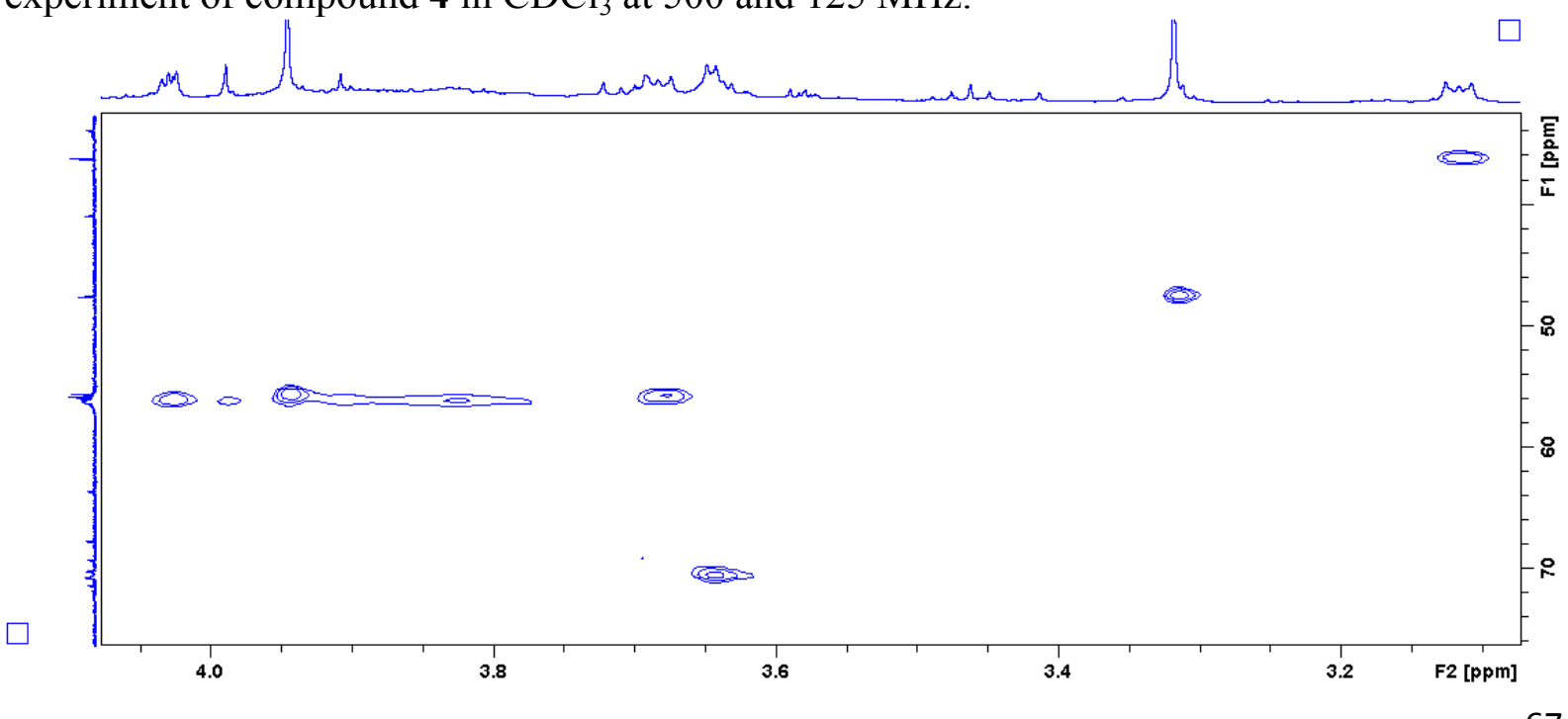


Figure S74. ${ }^{1} \mathrm{H}-{ }^{13} \mathrm{C}$ long-range correlation map from HMBC NMR experiment of compound 4 in $\mathrm{CDCl}_{3}$ at 500 and $125 \mathrm{MHz}$.

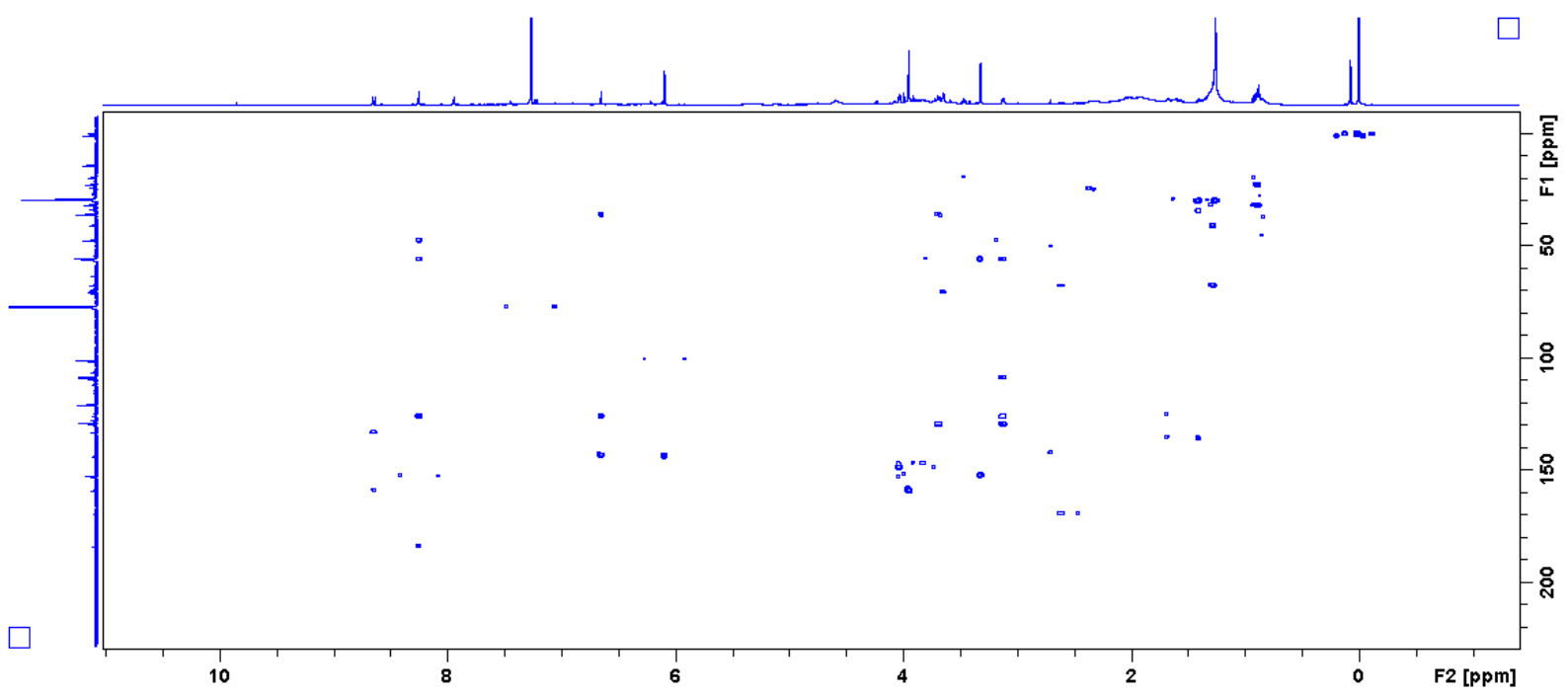

Figure S75. Enlargement $\left(\delta\right.$ 8.64-6.08) of ${ }^{1} \mathrm{H}^{-13} \mathrm{C}$ long-range correlation map from HMBC $\mathrm{NMR}$ experiment of compound 4 in $\mathrm{CDCl}_{3}$ at 500 and $125 \mathrm{MHz}$.

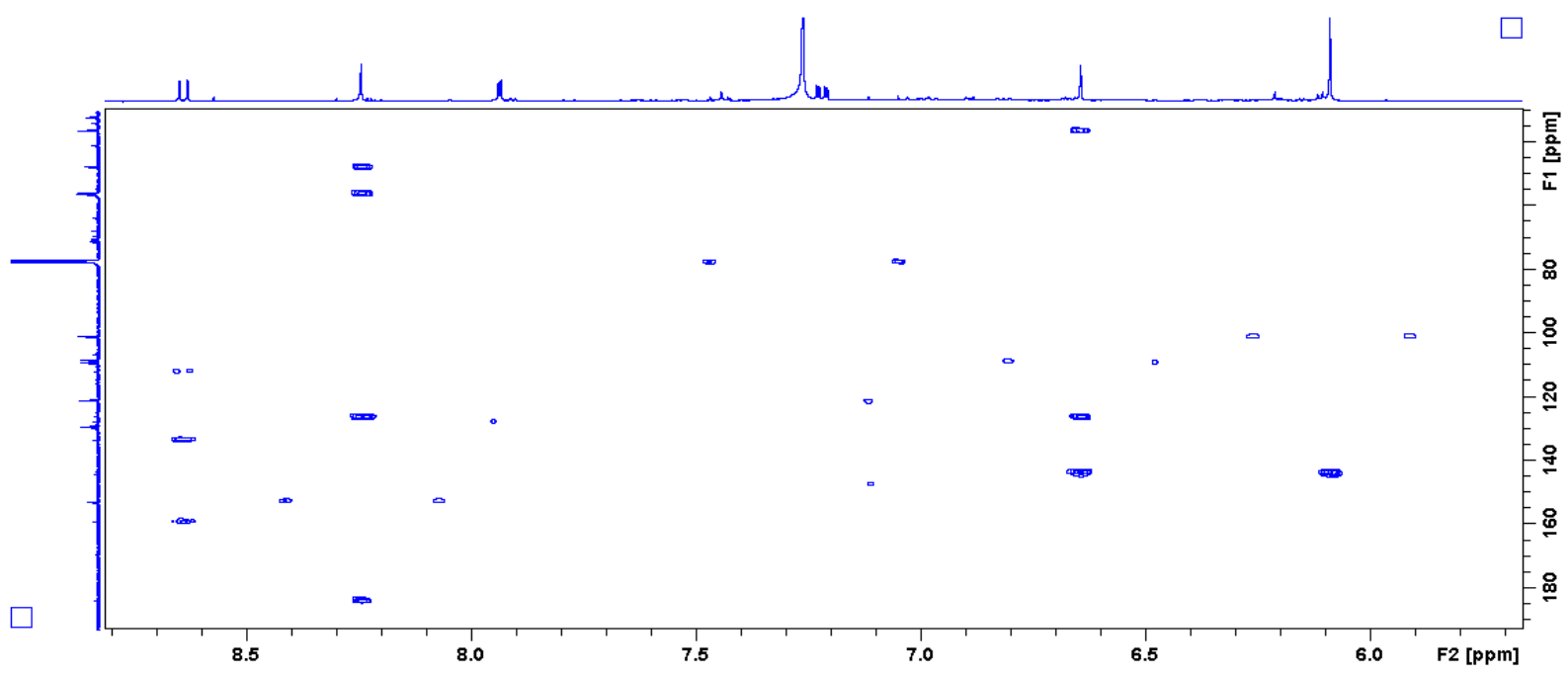


Figure S76. Enlargement $(\delta 3.94-3.11)$ of ${ }^{1} \mathrm{H}-{ }^{13} \mathrm{C}$ long-range correlation map from HMBC NMR experiment of compound 4 in $\mathrm{CDCl}_{3}$ at 500 and $125 \mathrm{MHz}$.

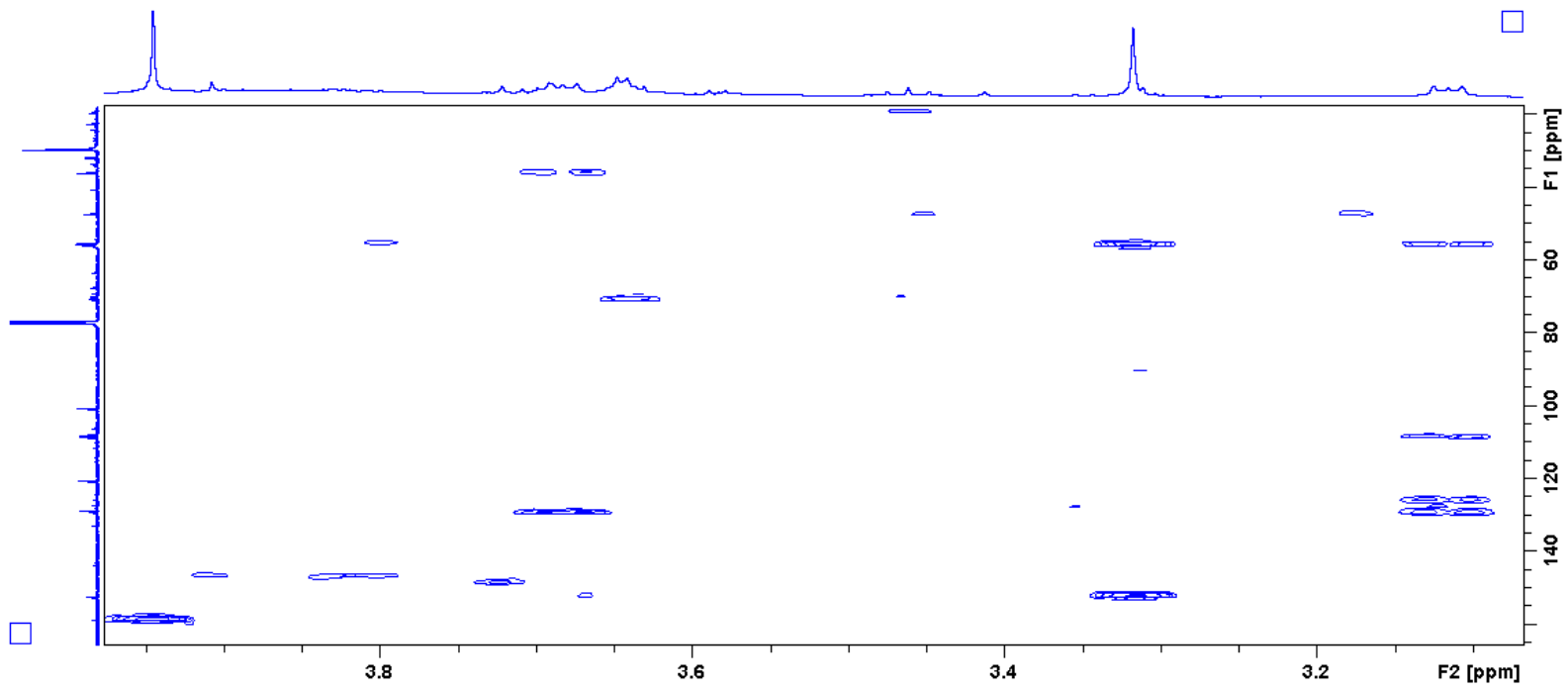


Figure S77. HRESIMS spectrum of compound 4.

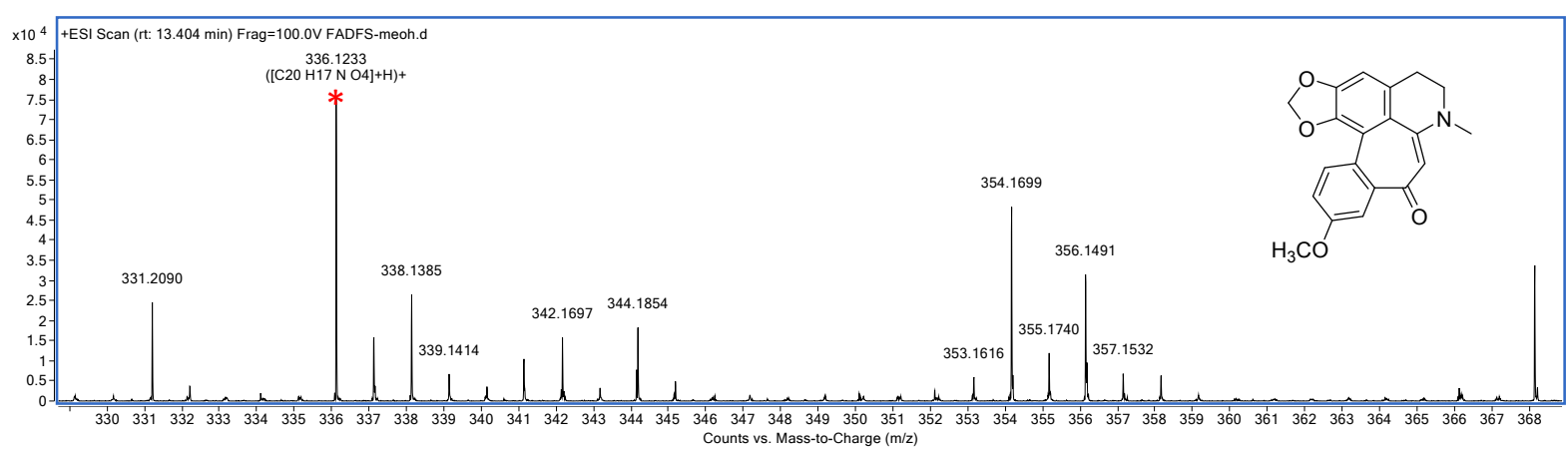


Figure S78. ESIMS/MS spectrum of compound 4.

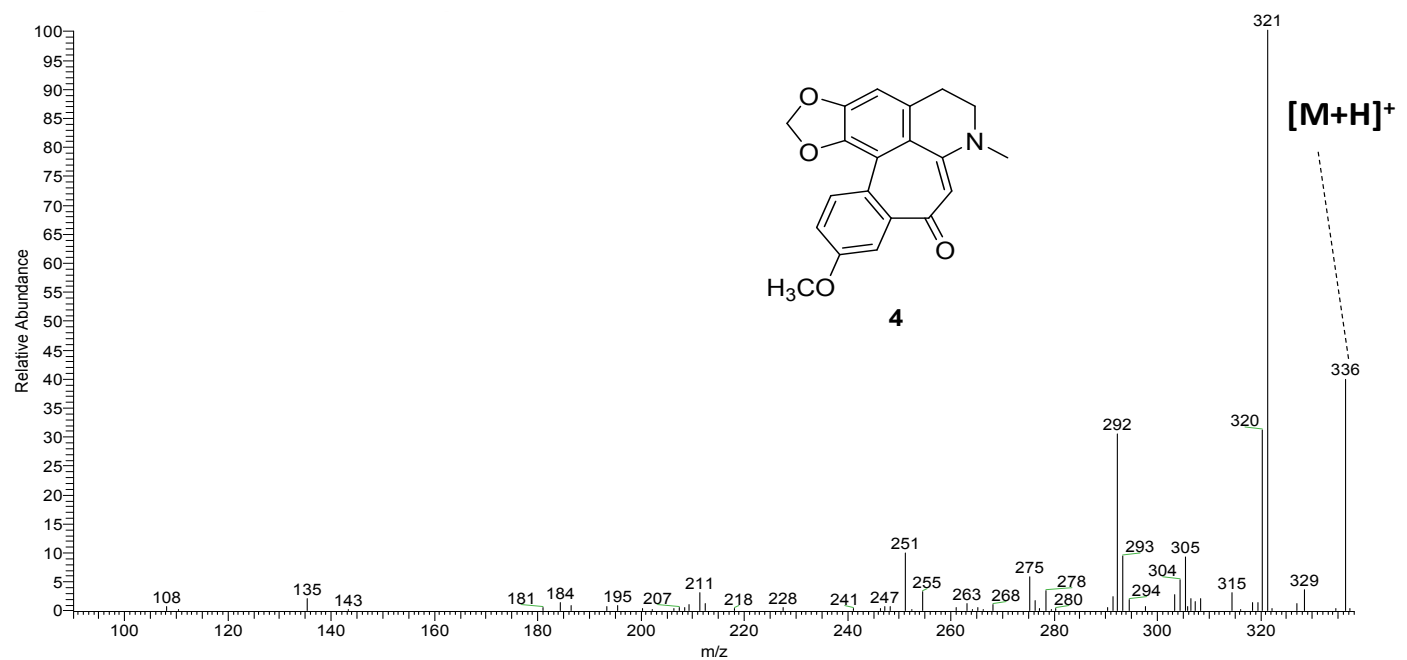


Figure S79. ${ }^{1} \mathrm{H}$ NMR spectrum of compound 5 in $\mathrm{CDCl}_{3}$ at $500 \mathrm{MHz}$. 


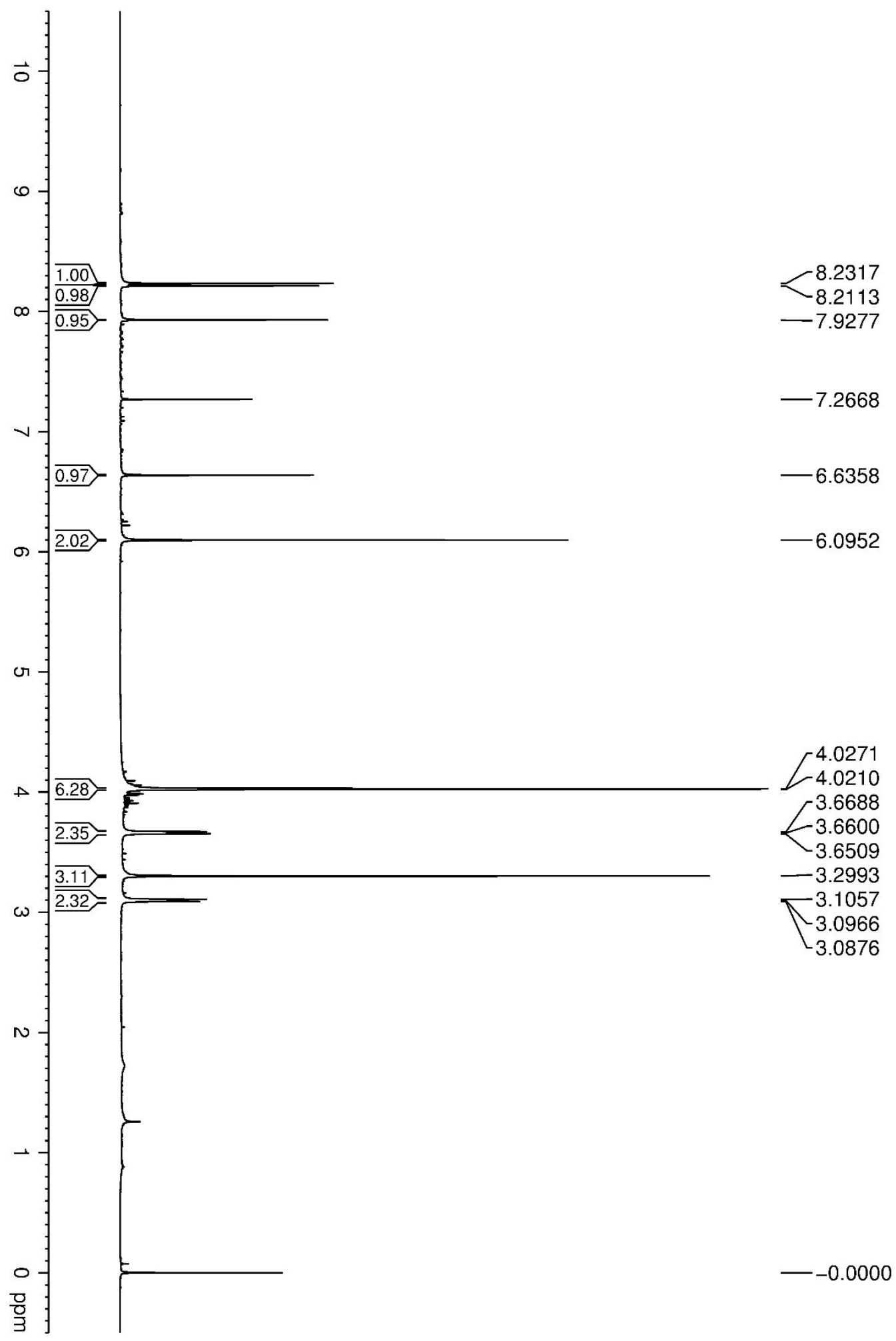


Figure S80. Enlargement ( $\delta$ 8.23-6.09) of ${ }^{1} \mathrm{H}$ NMR spectrum of compound $\mathbf{5}$ in $\mathrm{CDCl}_{3}$ at 500 $\mathrm{MHz}$.

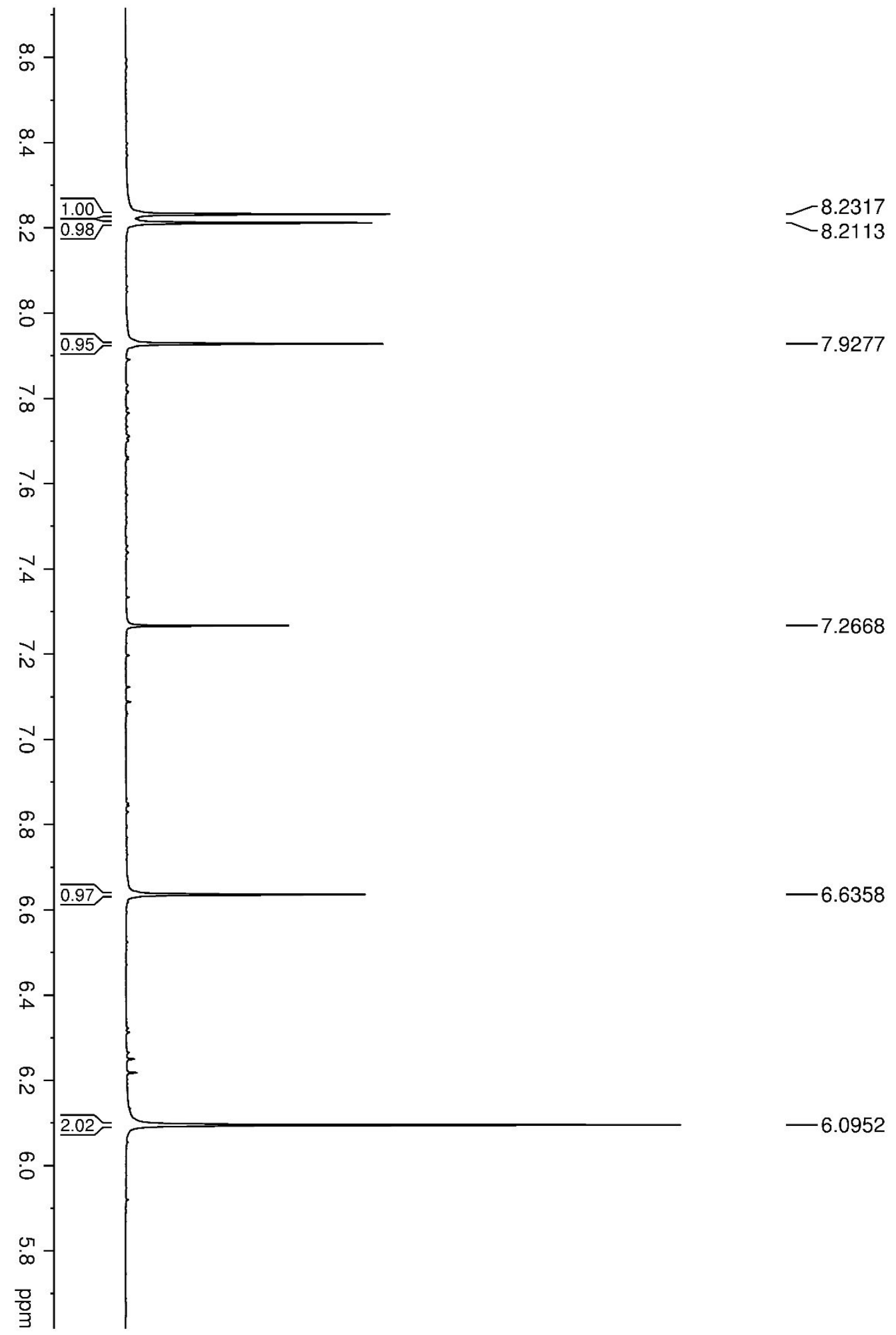


Figure S81. Enlargement $\left(\delta\right.$ 4.02-3.08) of ${ }^{1} \mathrm{H}$ NMR spectrum of compound $\mathbf{5}$ in $\mathrm{CDCl}_{3}$ at 500 $\mathrm{MHz}$.

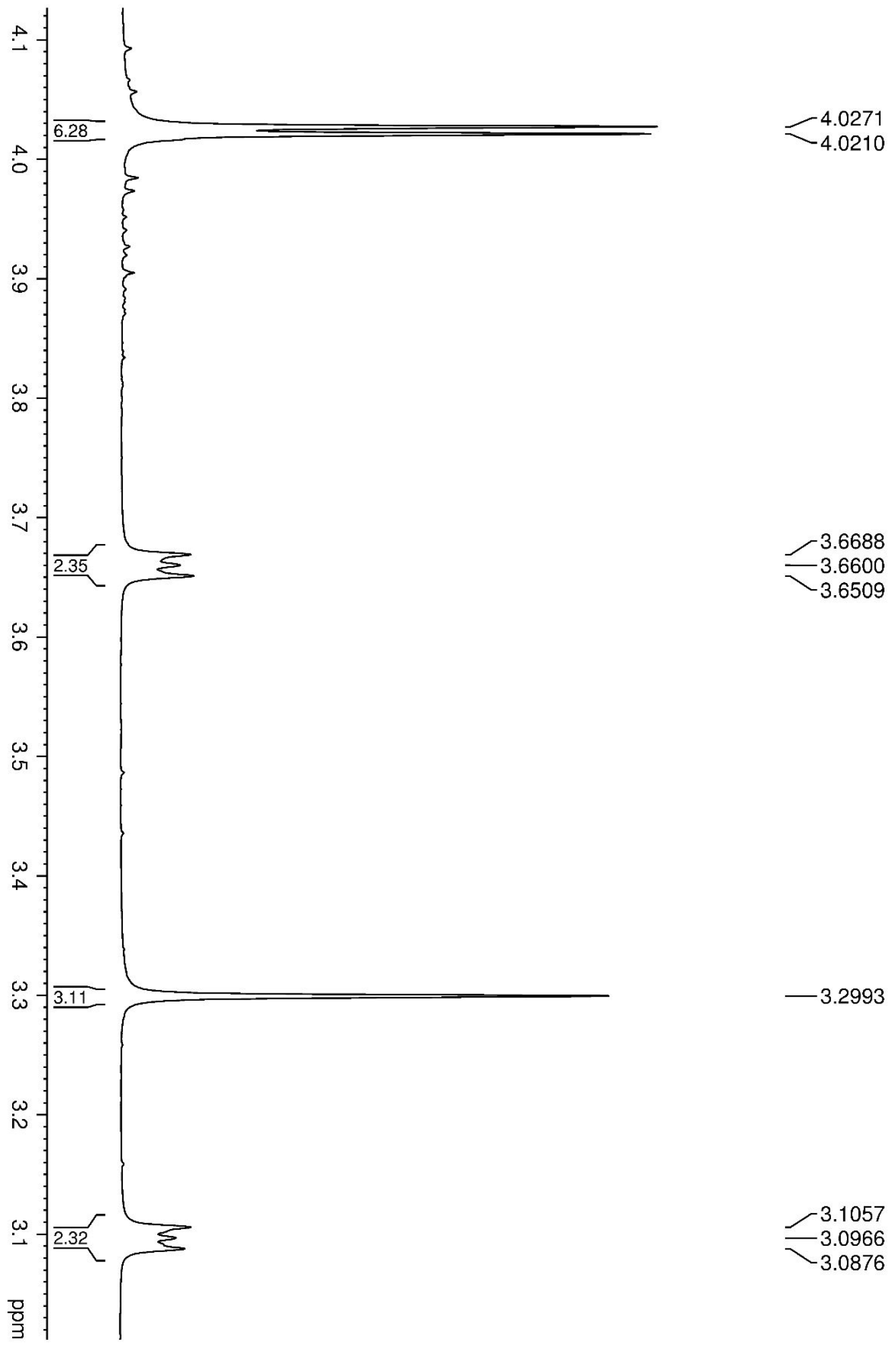


Figure S82. ${ }^{13} \mathrm{C}$ NMR spectrum of compound 5 in $\mathrm{CDCl}_{3}$ at $125 \mathrm{MHz}$.

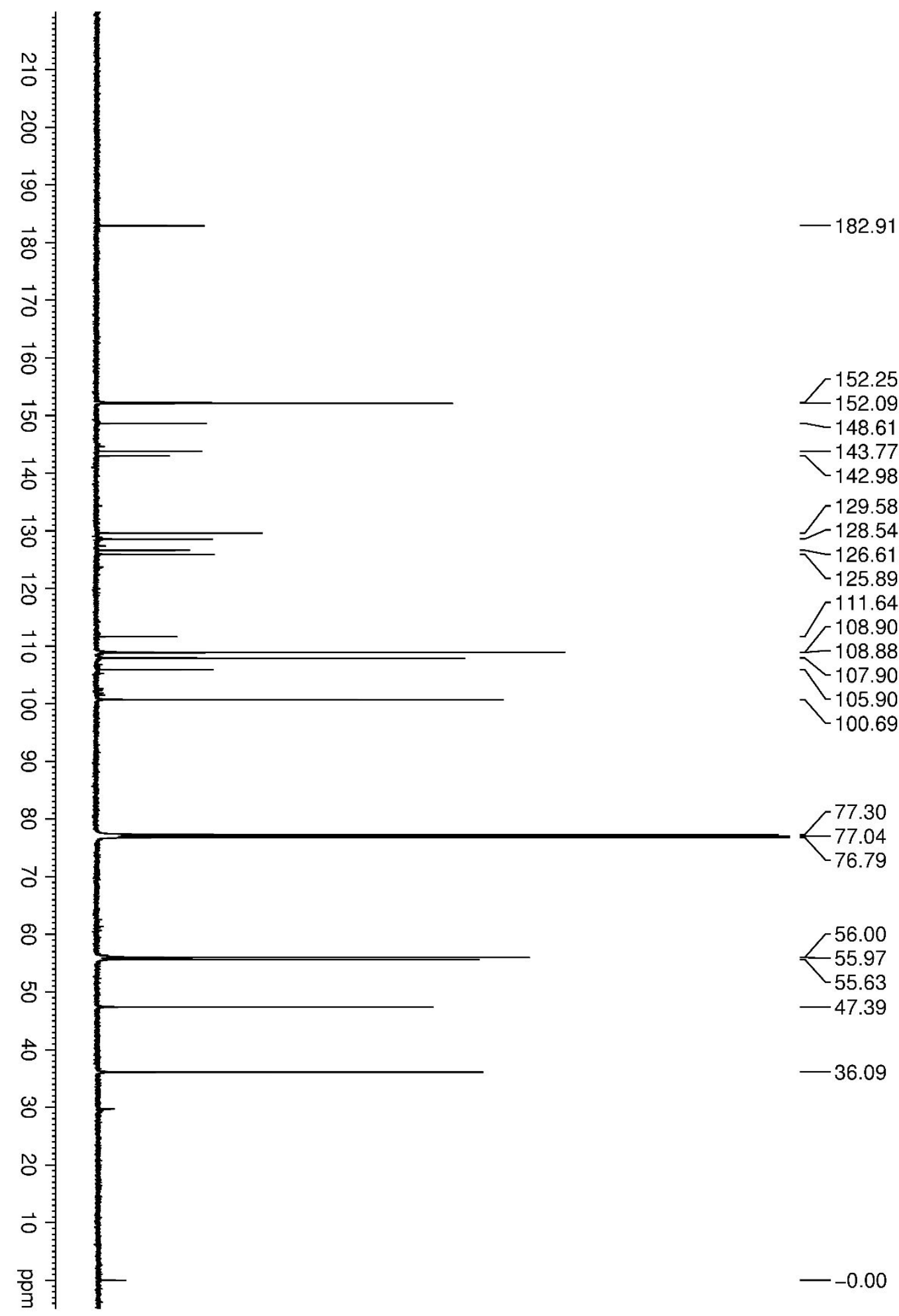


Figure S83. Enlargement ( $\delta$ 182.9-125.8) of ${ }^{13} \mathrm{C}$ NMR spectrum of compound $\mathbf{5}$ in $\mathrm{CDCl}_{3}$ at $125 \mathrm{MHz}$.

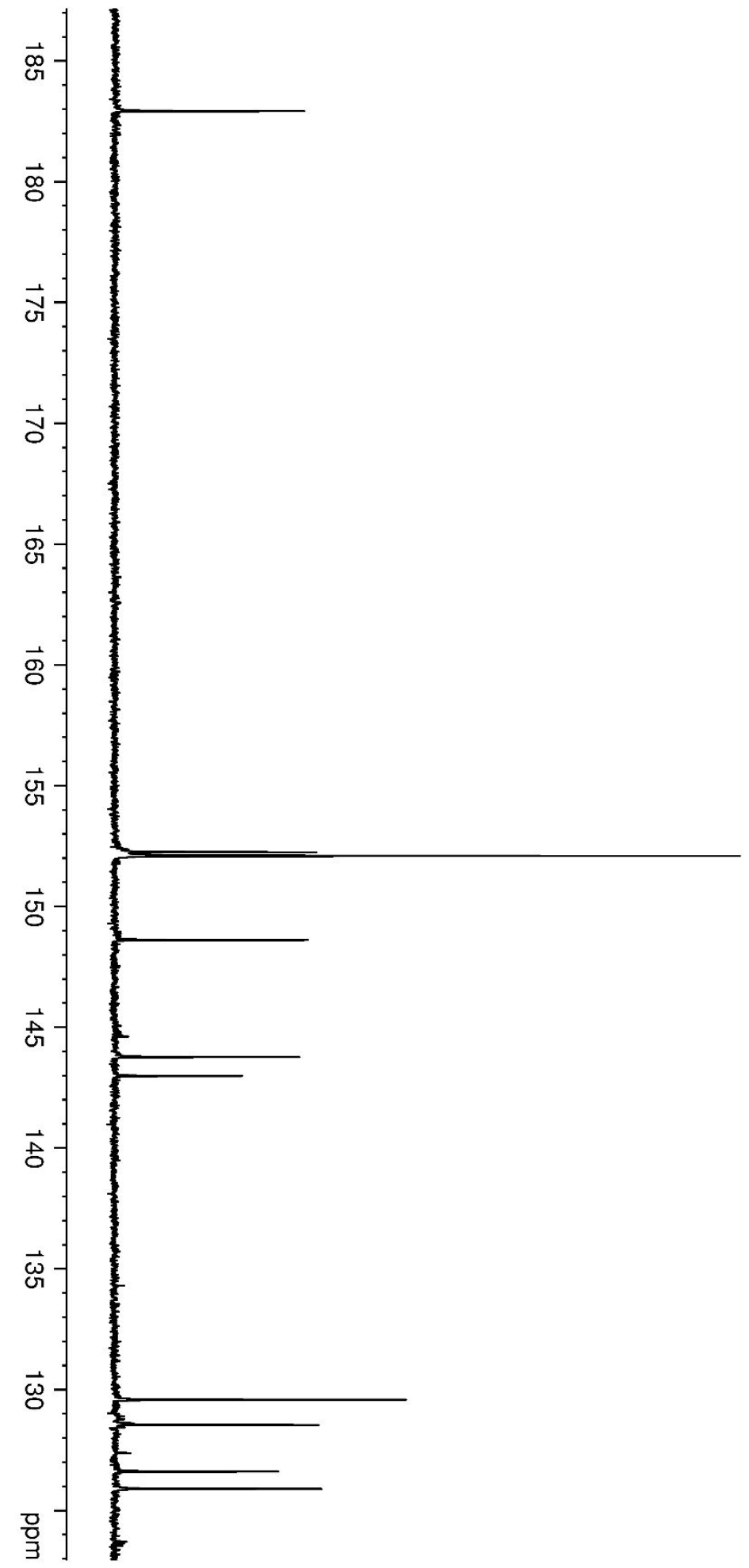


Figure S84. Enlargement $(\delta 111.6-36.0)$ of ${ }^{13} \mathrm{C}$ NMR spectrum of compound 5 in $\mathrm{CDCl}_{3}$ at 125 $\mathrm{MHz}$.

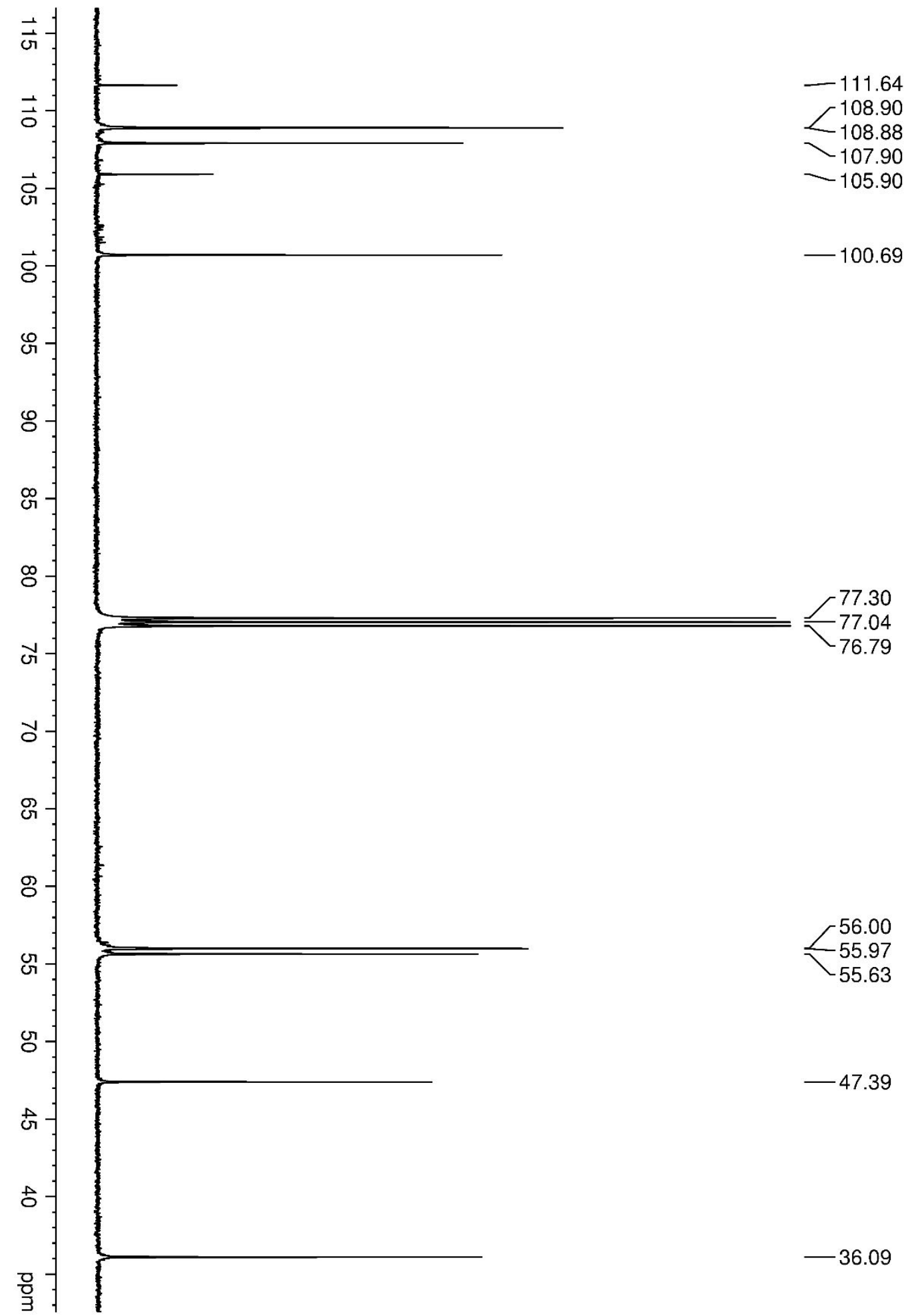


Figure S85. ${ }^{13} \mathrm{C}$ NMR DEP135 spectrum of compound 5 in $\mathrm{CDCl}_{3}$ at $125 \mathrm{MHz}$.

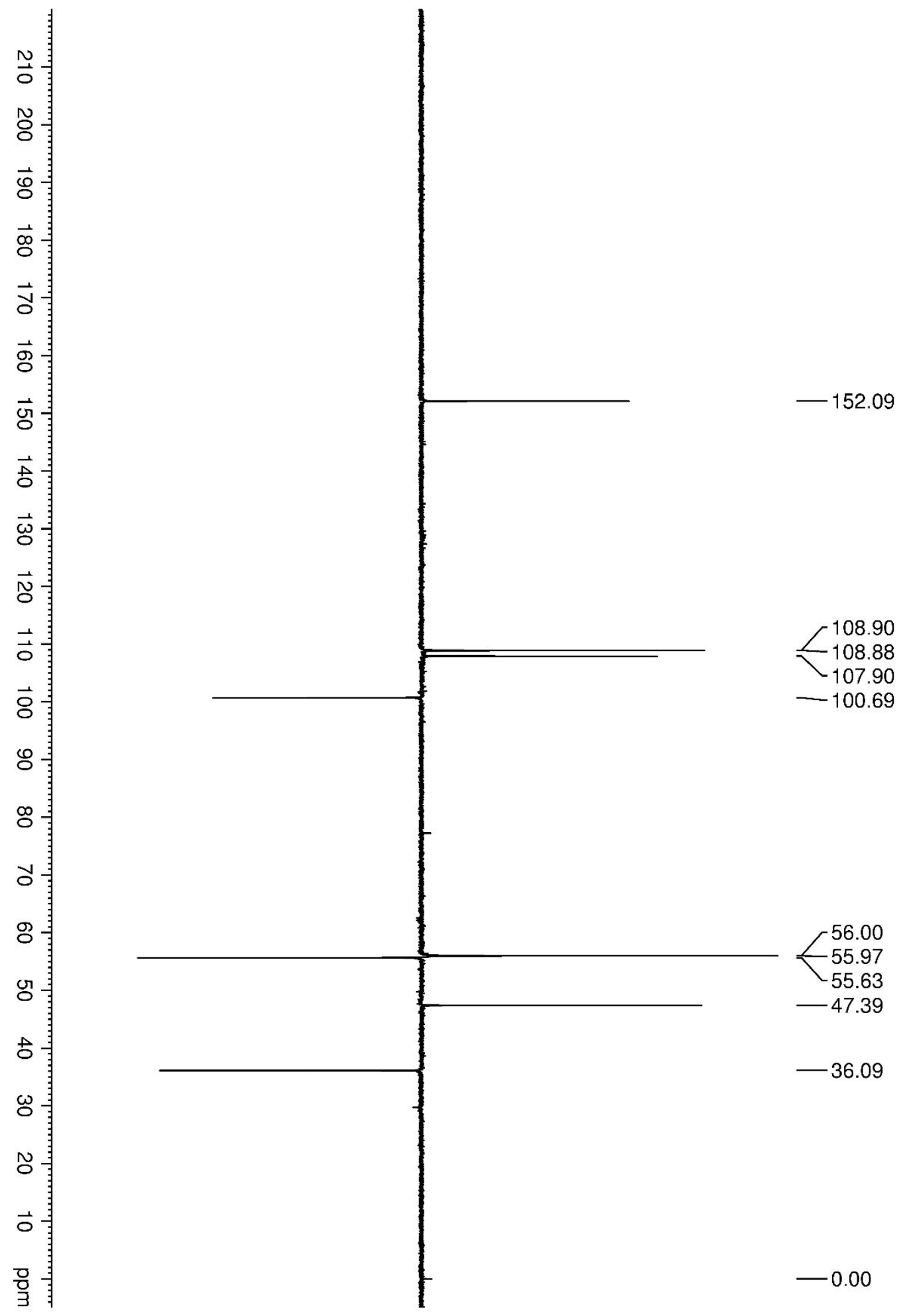


Figure S86. Enlargement $(\delta 152.0-100.6)$ of ${ }^{13} \mathrm{C}$ NMR DEP135 spectrum of compound 5 in $\mathrm{CDCl}_{3}$ at $125 \mathrm{MHz}$.

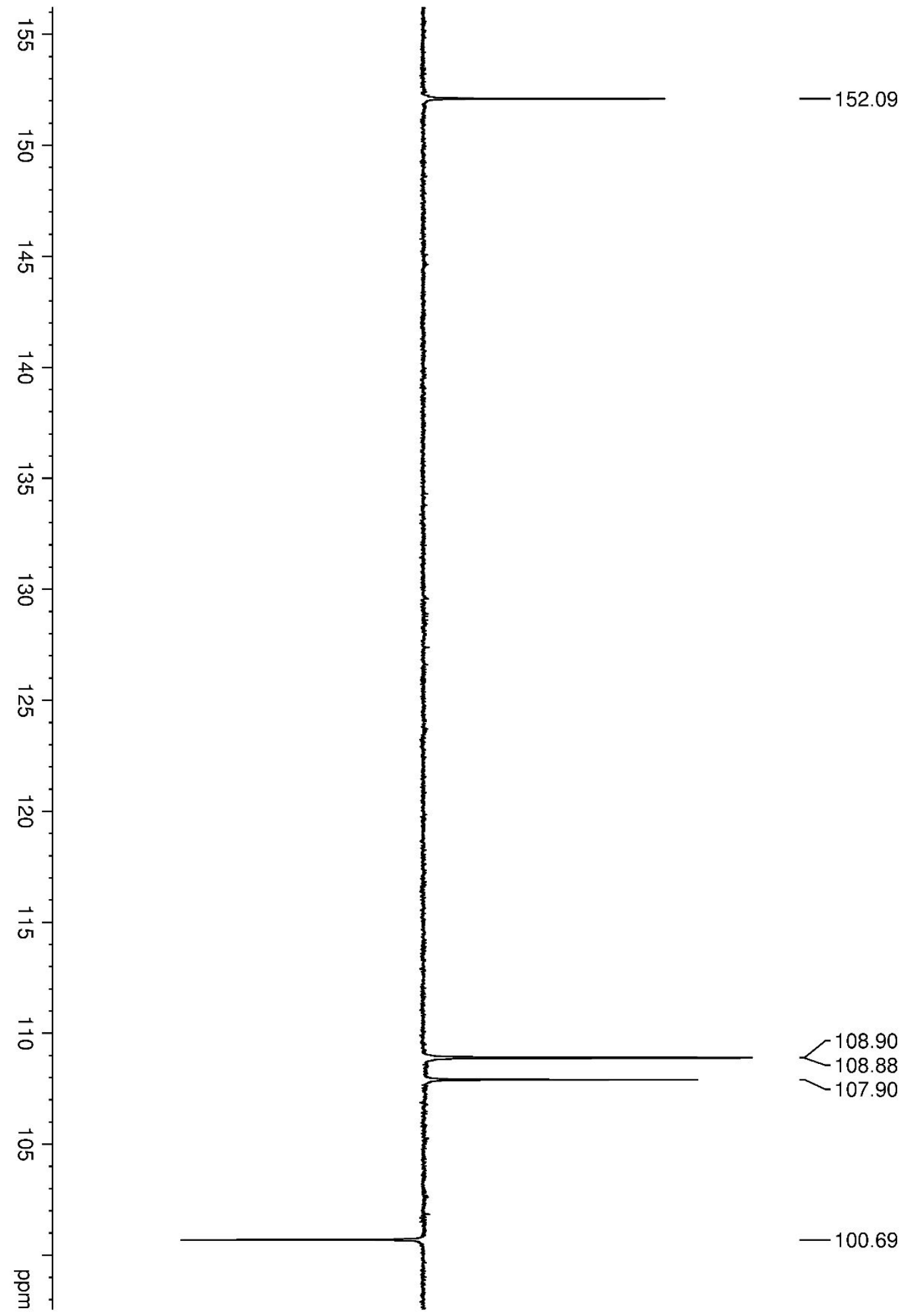


Figure S87. Enlargement $(\delta 56.0-36.0)$ of ${ }^{13} \mathrm{C}$ NMR DEP135 spectrum of compound 5 in $\mathrm{CDCl}_{3}$ at $125 \mathrm{MHz}$.

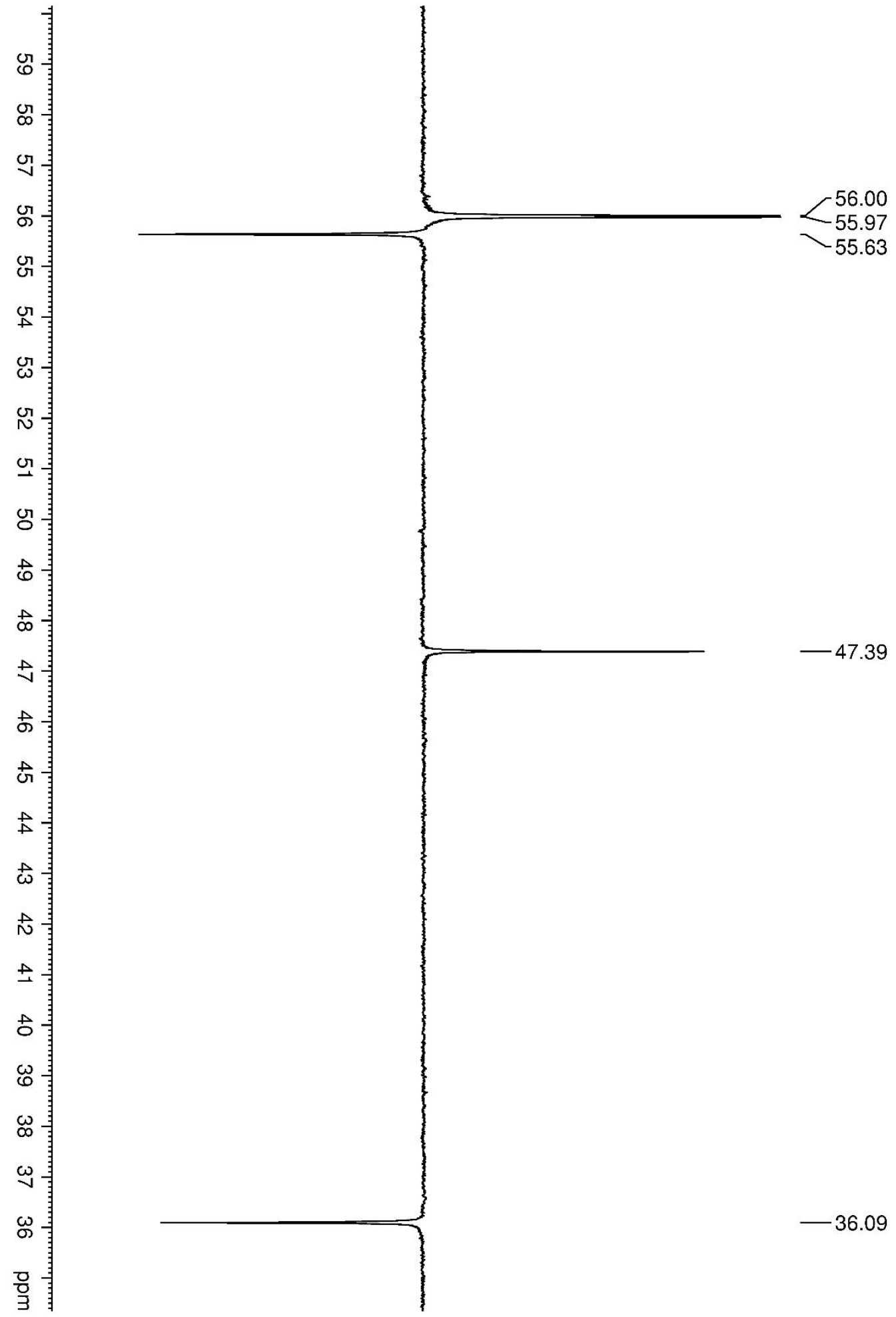


Figure S88. ${ }^{1} \mathrm{H}-{ }^{1} \mathrm{H}$ COSY correlation map from ${ }^{1} \mathrm{H}$ NMR experiment of compound 5 in $\mathrm{CDCl}_{3}$ at $500 \mathrm{MHz}$.

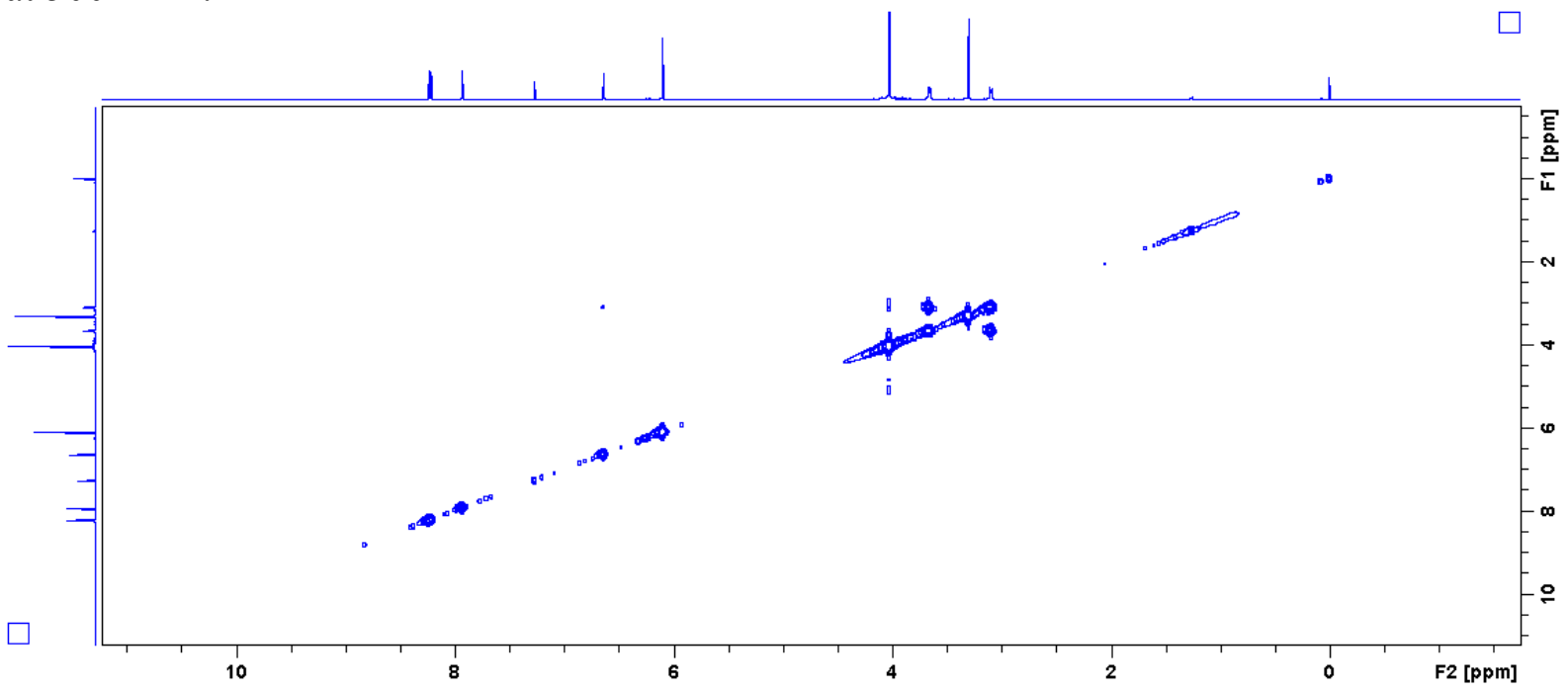

Figure S89. Enlargement $\left(\delta\right.$ 8.23-6.09) of ${ }^{1} \mathrm{H}-{ }^{1} \mathrm{H}$ COSY correlation map from ${ }^{1} \mathrm{H}$ NMR experiment of compound 5 in $\mathrm{CDCl}_{3}$ at $500 \mathrm{MHz}$.

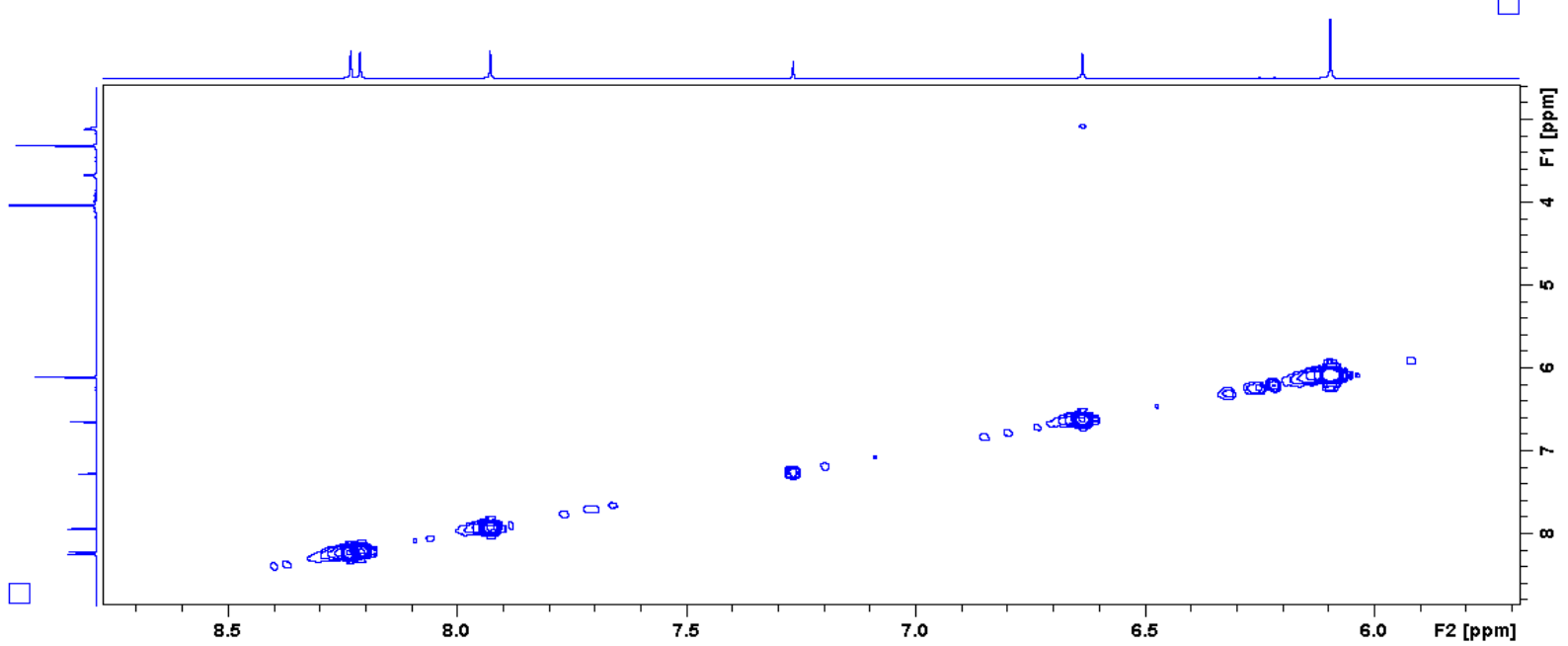

Figure S90. Enlargement $\left(\delta\right.$ 4.02-3.08) of ${ }^{1} \mathrm{H}-{ }^{1} \mathrm{H}$ COSY correlation map from ${ }^{1} \mathrm{H}$ NMR experiment of compound 5 in $\mathrm{CDCl}_{3}$ at $500 \mathrm{MHz}$.

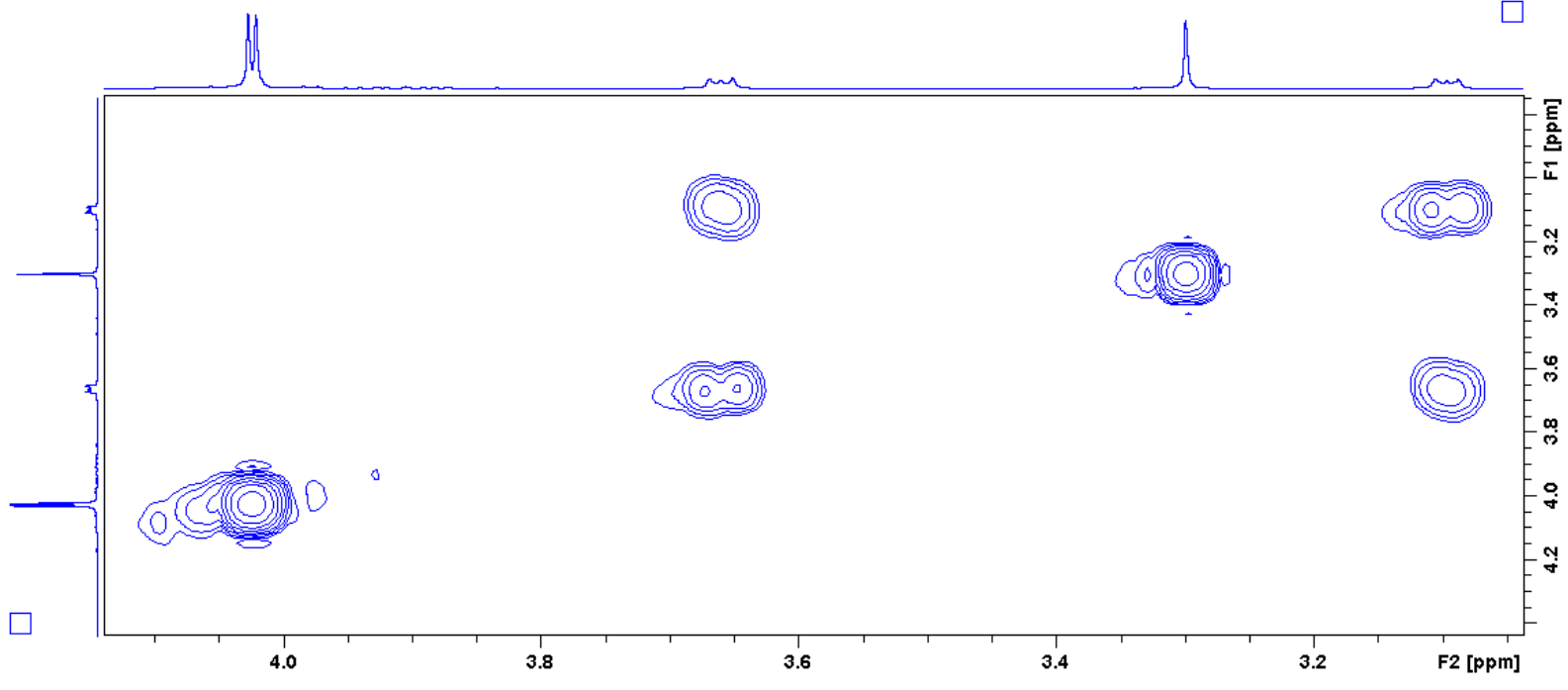


Figure S91. ${ }^{1} \mathrm{H}-{ }^{13} \mathrm{C}$ one-bond correlation map from HSQC NMR experiment of compound 5 in $\mathrm{CDCl}_{3}$ at 500 and $125 \mathrm{MHz}$.

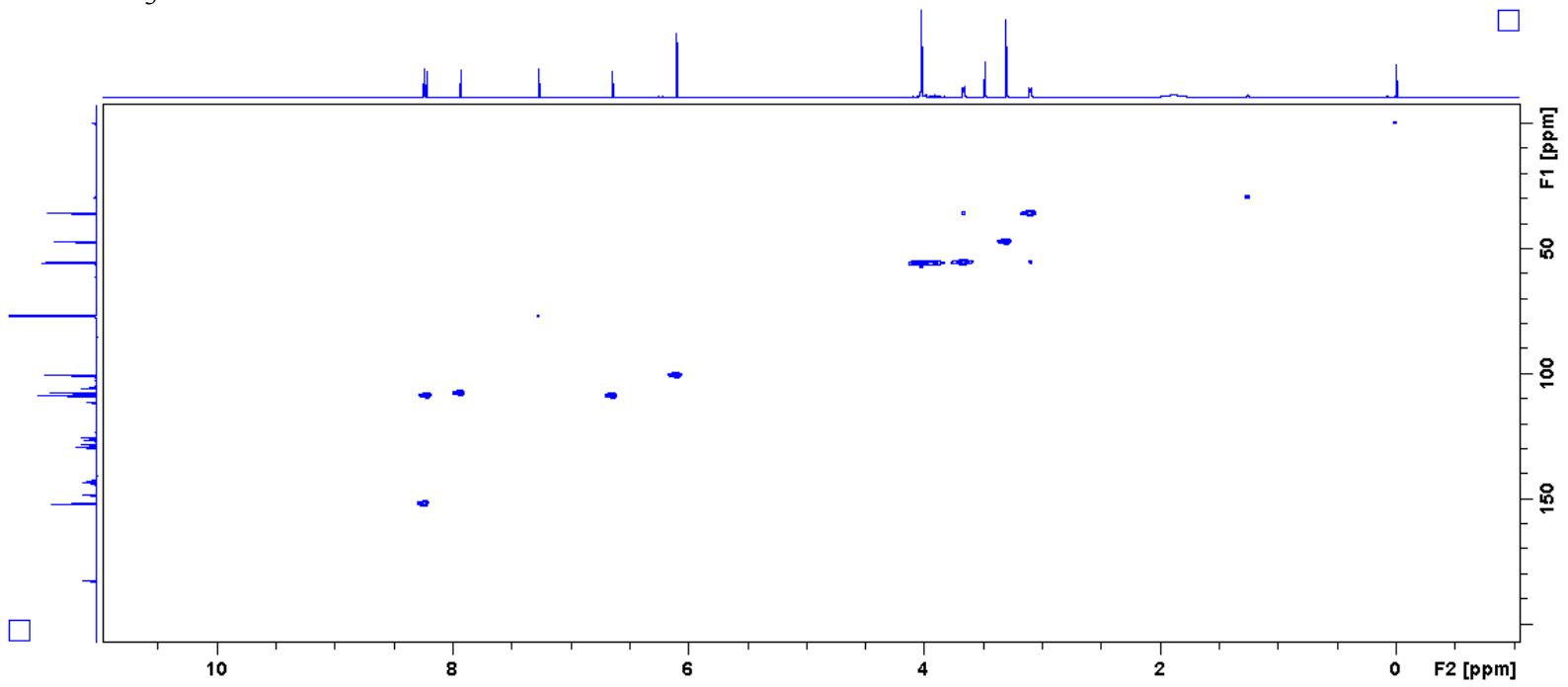

Figure S92. Enlargement $(\delta 8.23-6.09)$ of ${ }^{1} \mathrm{H}-{ }^{13} \mathrm{C}$ one-bond correlation map from HSQC NMR experiment of compound 5 in $\mathrm{CDCl}_{3}$ at 500 and $125 \mathrm{MHz}$.

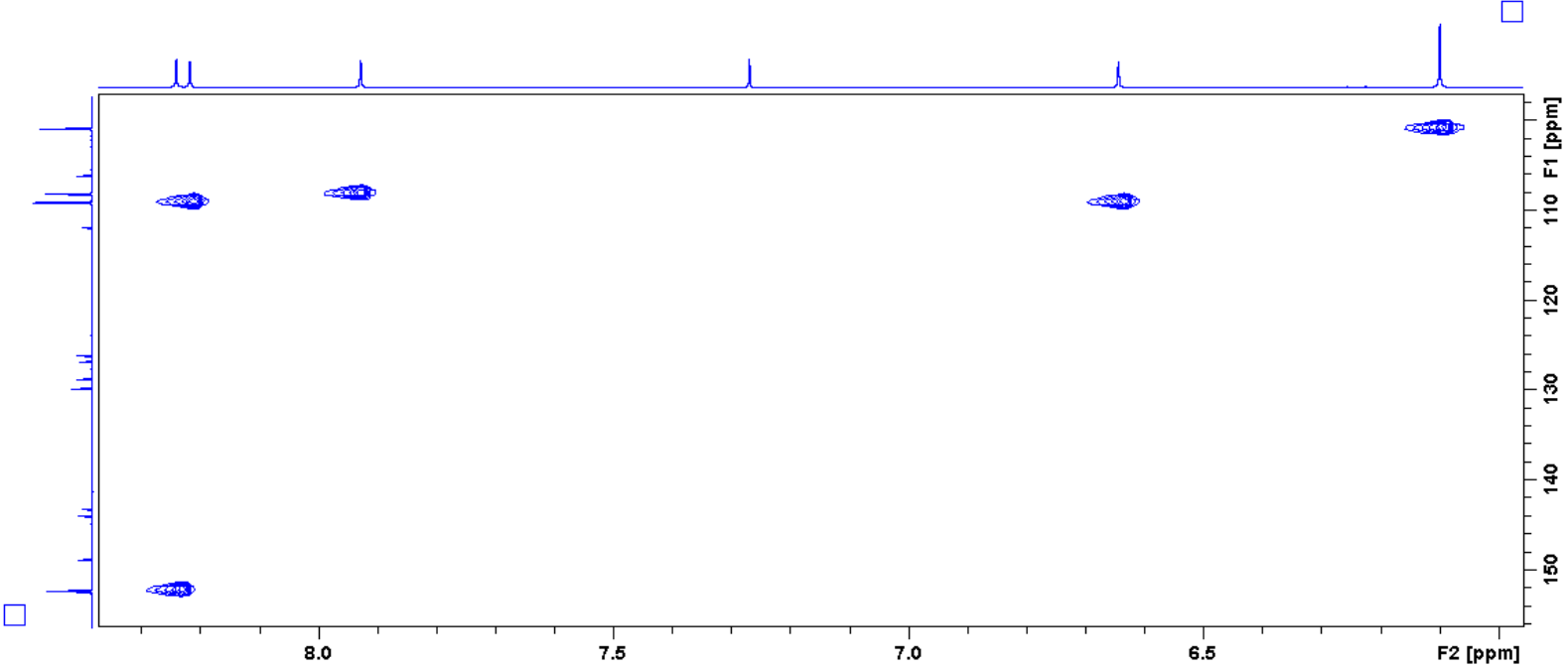

Figure S93. Enlargement $(\delta 4.02-3.08)$ of ${ }^{1} \mathrm{H}-{ }^{13} \mathrm{C}$ one-bond correlation map from HSQC NMR experiment of compound $\mathbf{5}$ in $\mathrm{CDCl}_{3}$ at 500 and $125 \mathrm{MHz}$.

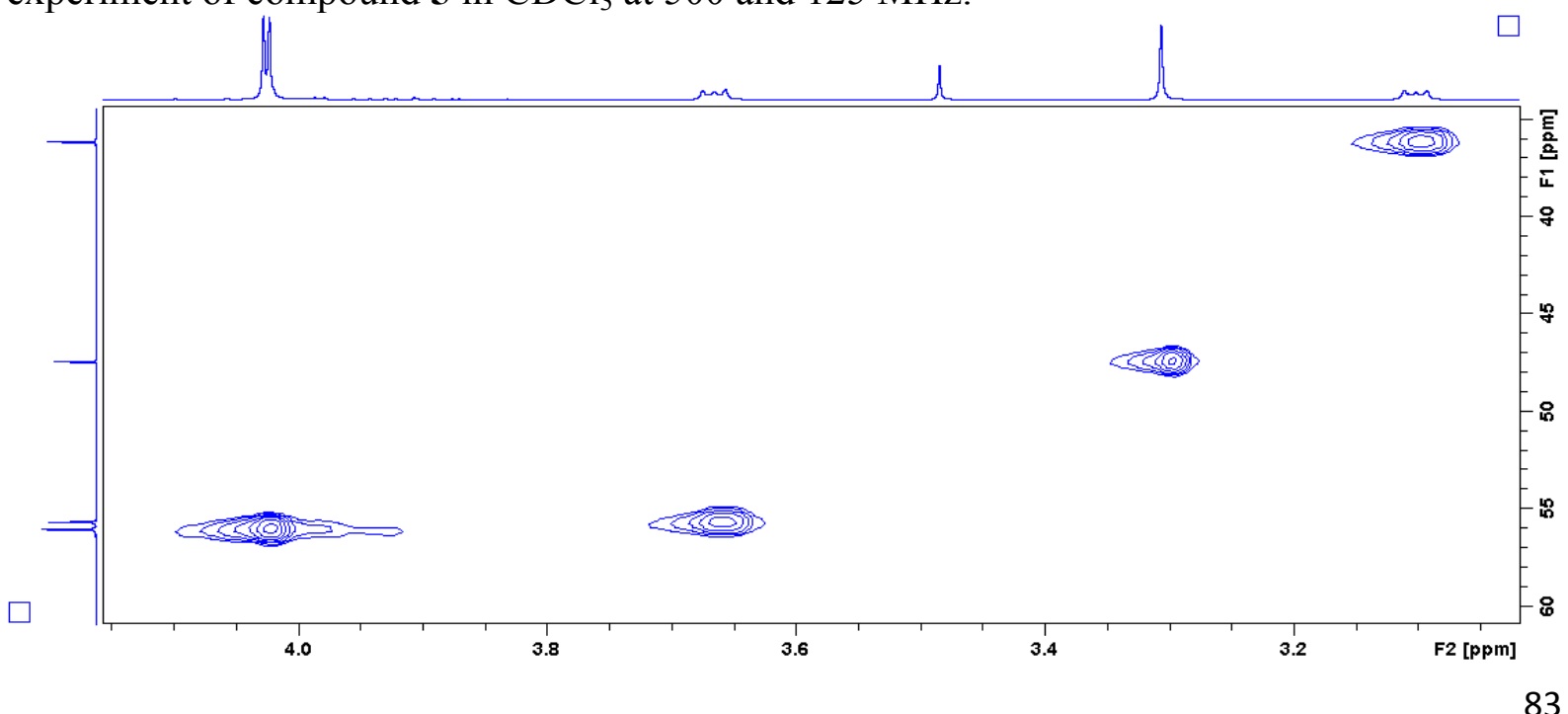


Figure S94. ${ }^{1} \mathrm{H}-{ }^{13} \mathrm{C}$ long-range correlation map from HMBC NMR experiment of compound 5 in $\mathrm{CDCl}_{3}$ at 500 and $125 \mathrm{MHz}$.

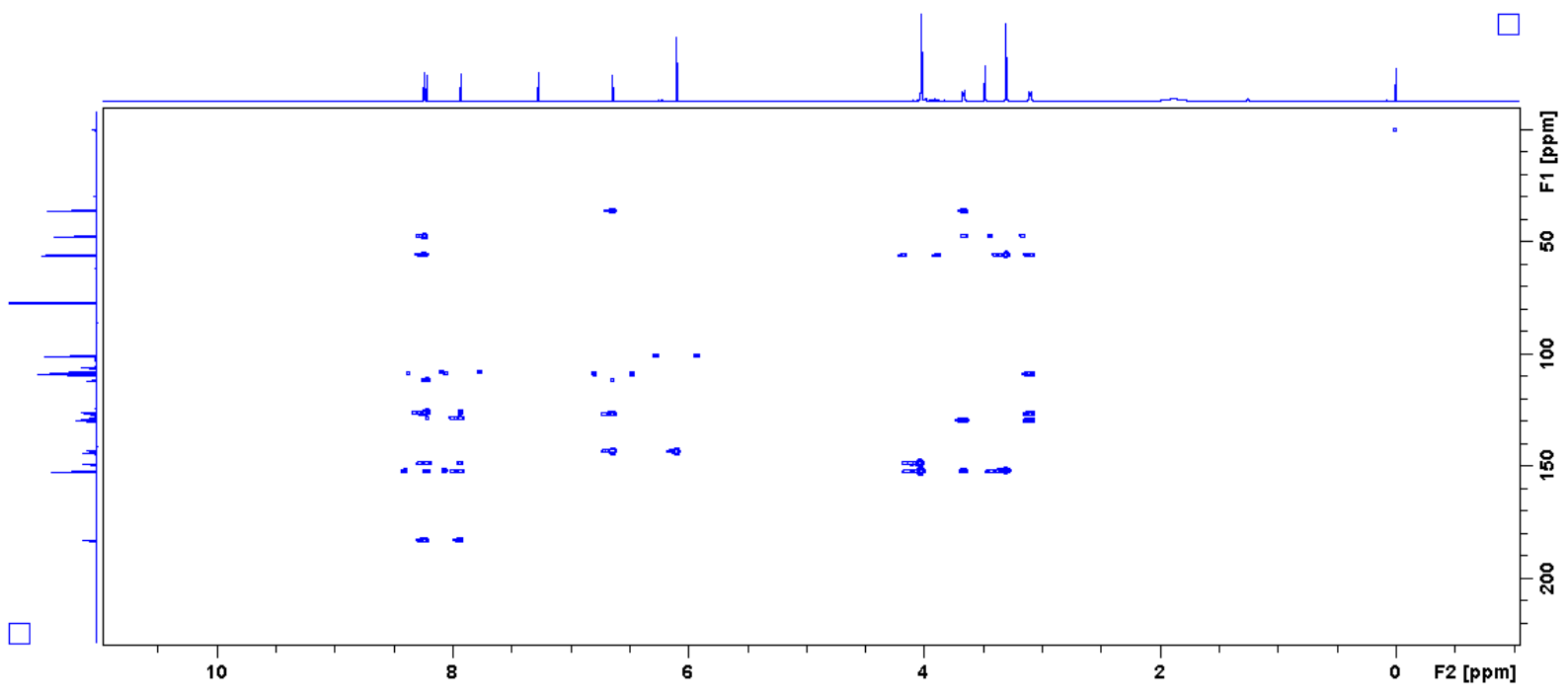

Figure S95. Enlargement $(\delta 8.23-6.09)$ of ${ }^{1} \mathrm{H}^{13} \mathrm{C}$ long-range correlation map from HMBC NMR experiment of compound 5 in $\mathrm{CDCl}_{3}$ at 500 and $125 \mathrm{MHz}$.

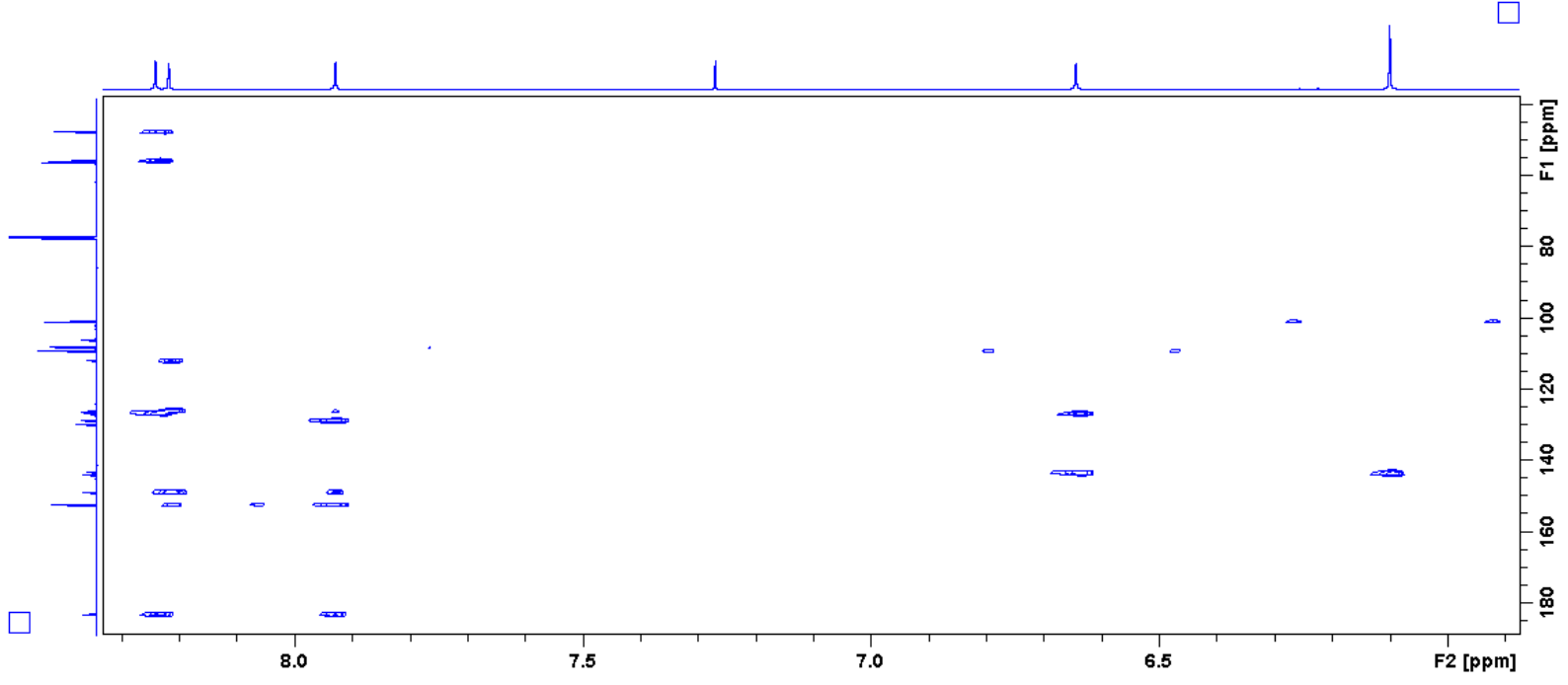


Figure S96. Enlargement $\left(\delta\right.$ 4.02-3.08) of ${ }^{1} \mathrm{H}-{ }^{13} \mathrm{C}$ long-range correlation map from HMBC NMR experiment of compound $\mathbf{5}$ in $\mathrm{CDCl}_{3}$ at 500 and $125 \mathrm{MHz}$.

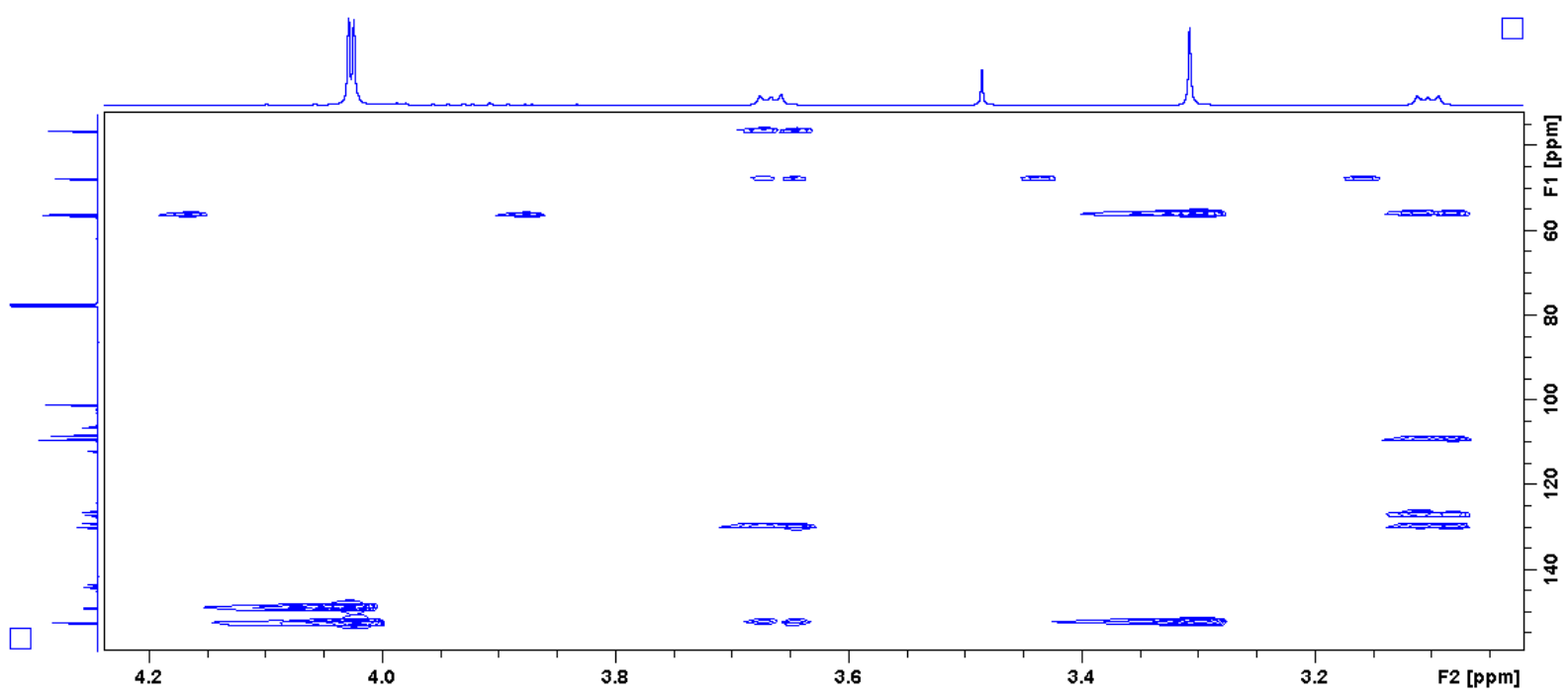


Figure S97. HRESIMS spectrum of compound $\mathbf{5}$.

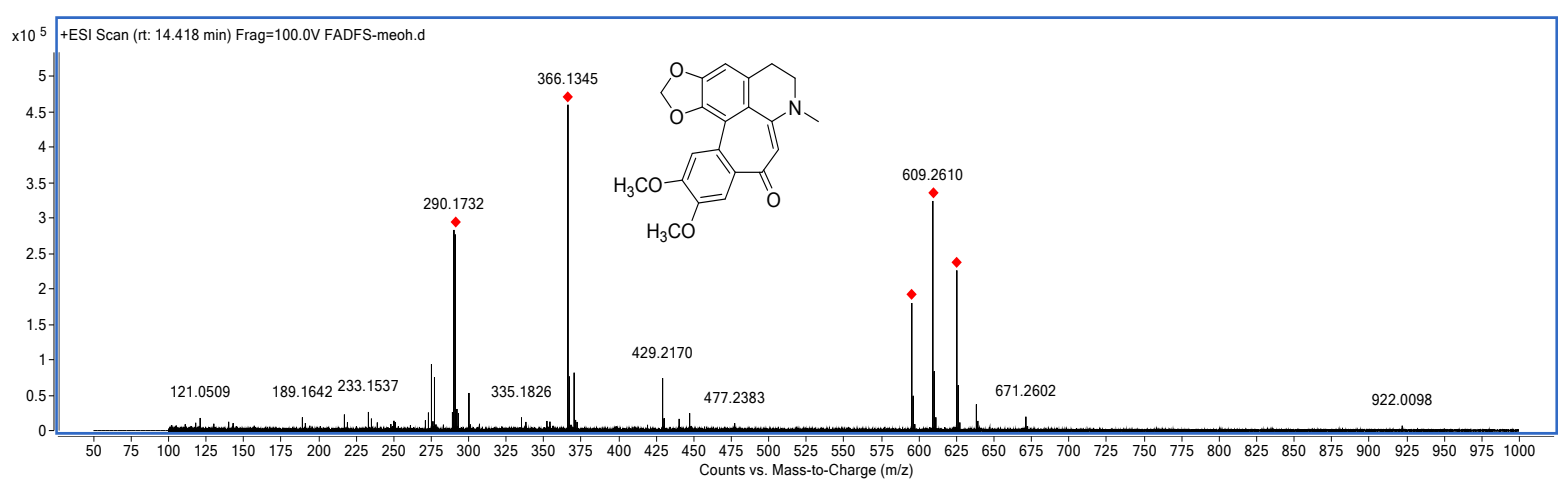


Figure S98. Extracted ion chromatogram, MS and MS/MS spectra of compound 5.
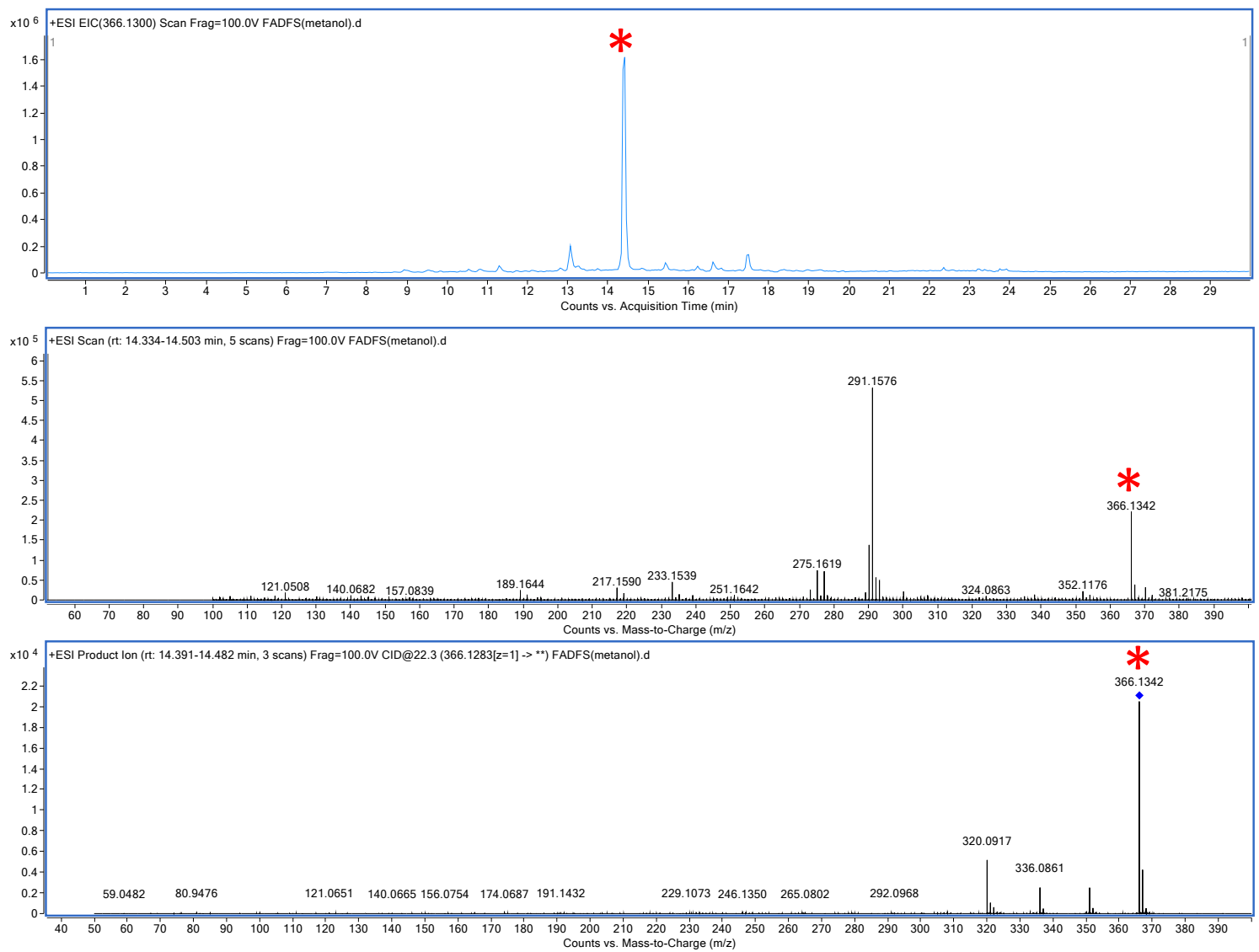
Figure S99. ${ }^{1} \mathrm{H}$ NMR spectrum of compound 10 in $\mathrm{CDCl}_{3}$ at $500 \mathrm{MHz}$.

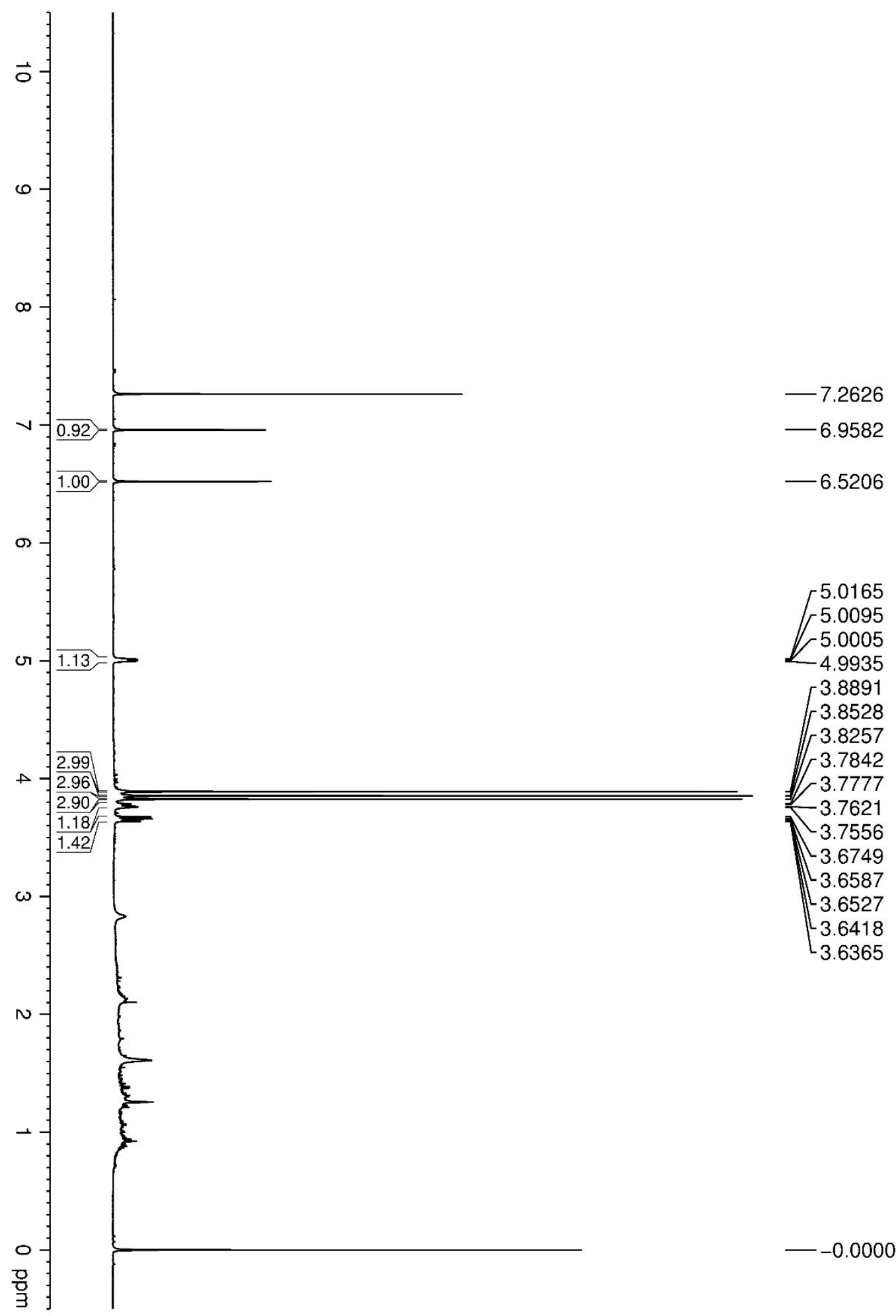


Figure S100. Enlargement $(\delta 7.26-6.52)$ of ${ }^{1} \mathrm{H}$ NMR spectrum of compound 10 in $\mathrm{CDCl}_{3}$ at $500 \mathrm{MHz}$.

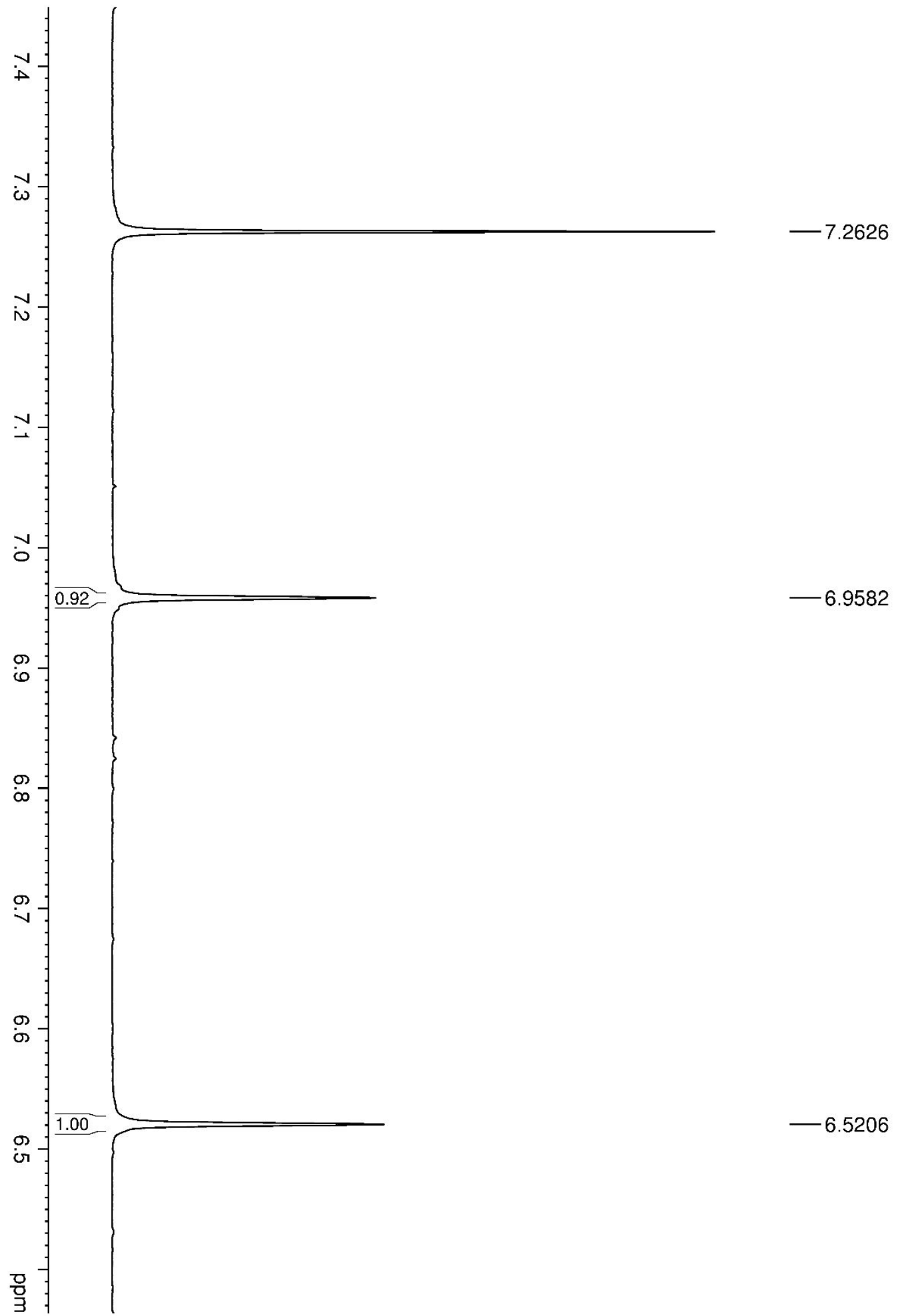


Figure S101. Enlargement ( $\delta 5.01-3.63)$ of ${ }^{1} \mathrm{H}$ NMR spectrum of compound 10 in $\mathrm{CDCl}_{3}$ at $500 \mathrm{MHz}$.

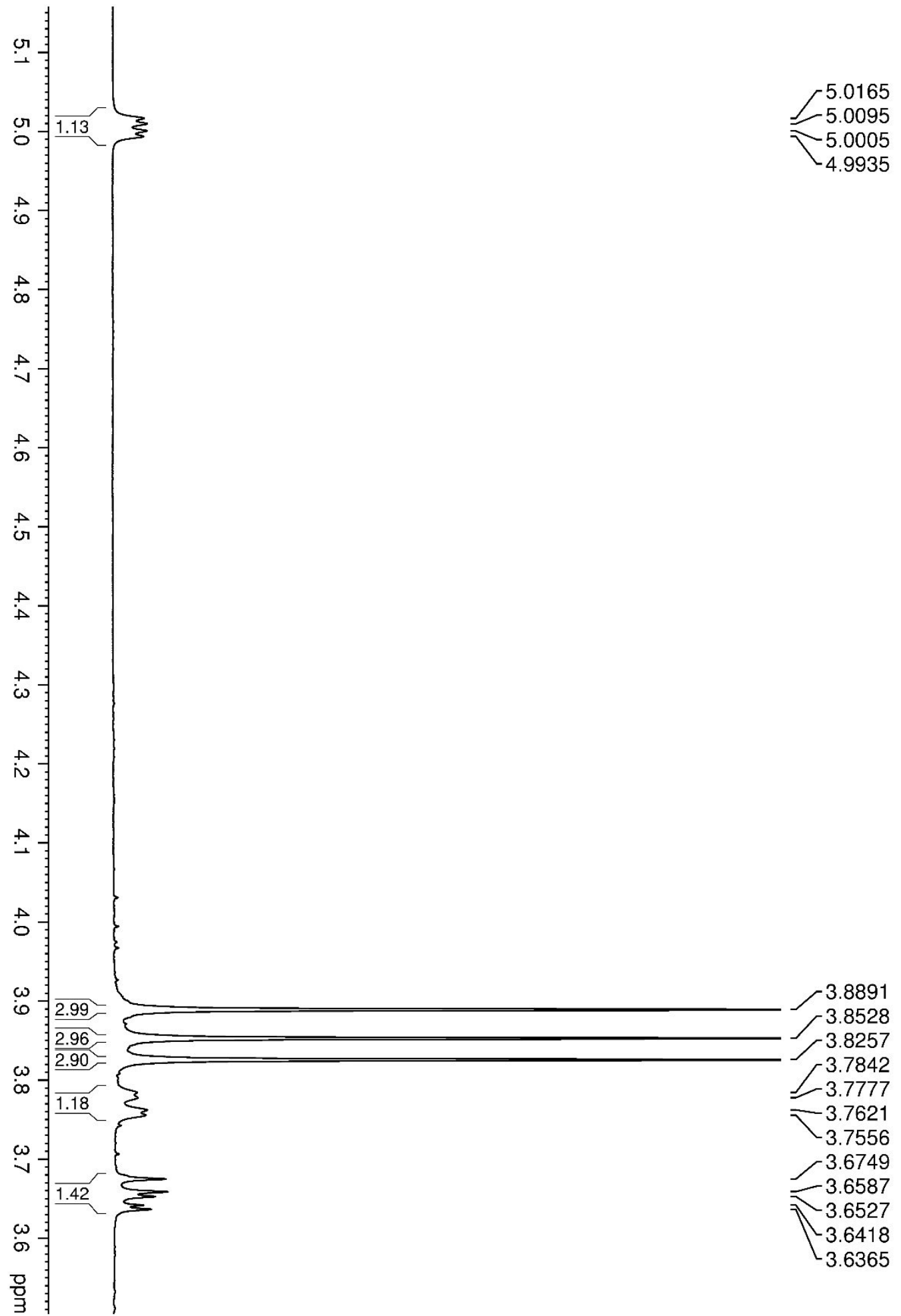


Figure S102. ${ }^{13} \mathrm{C}$ NMR spectrum of compound $\mathbf{1 0}$ in $\mathrm{CDCl}_{3}$ at $125 \mathrm{MHz}$.

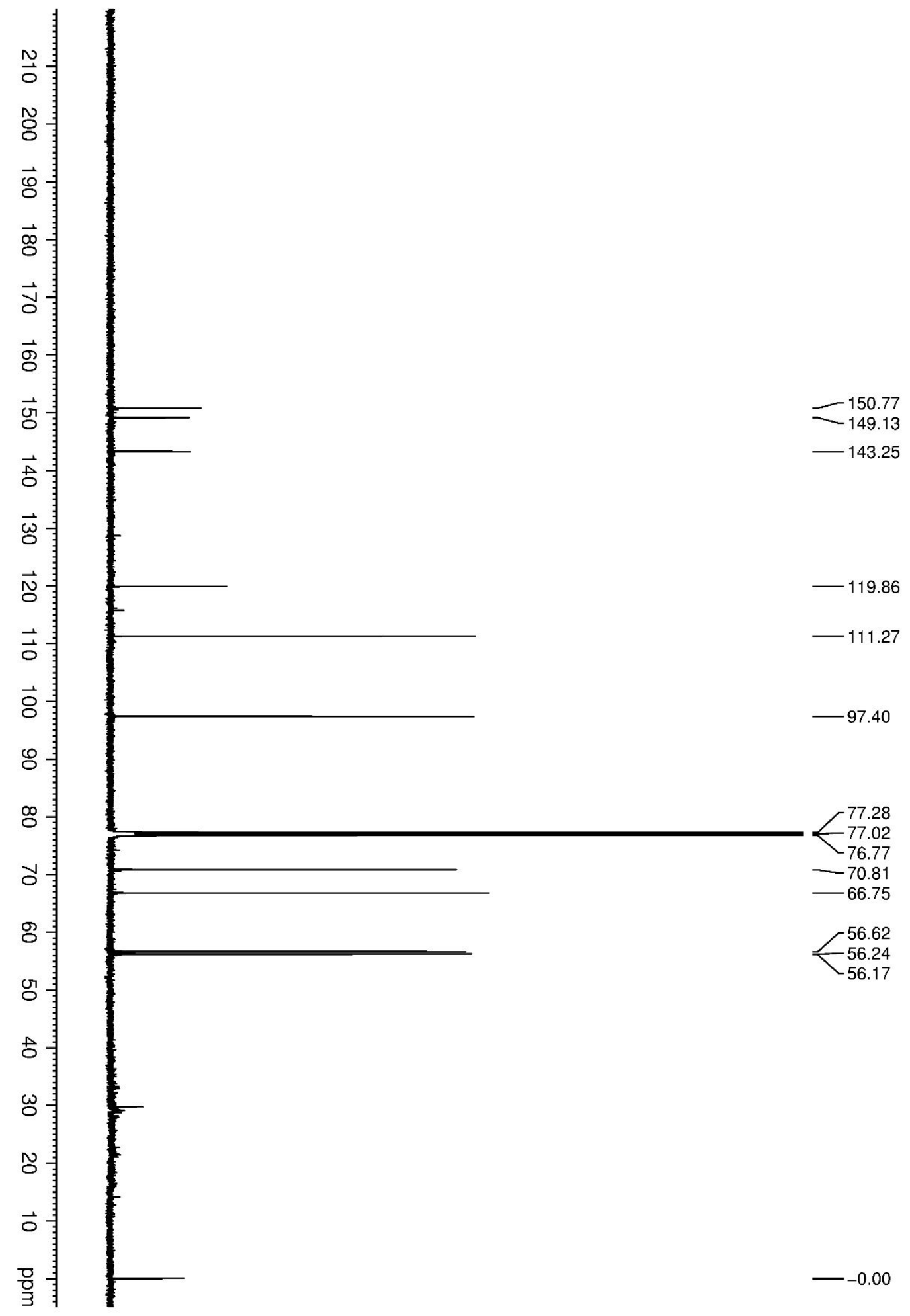


Figure S103. Enlargement $\left(\delta\right.$ 150.7-97.3) of ${ }^{13} \mathrm{C}$ NMR spectrum of compound 10 in $\mathrm{CDCl}_{3}$ at $125 \mathrm{MHz}$.

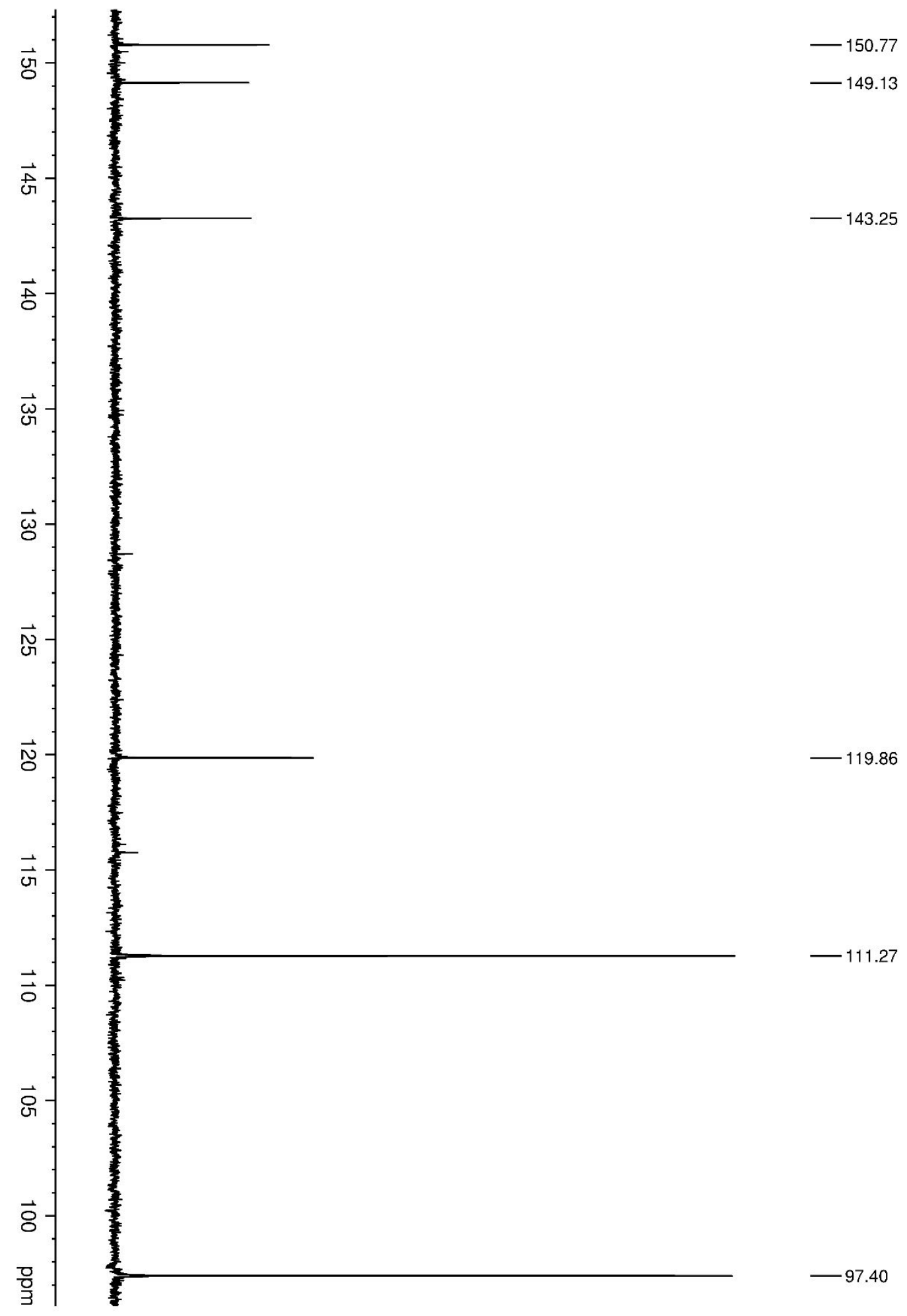


Figure S104. Enlargement $(\delta 70.8-56.1)$ of ${ }^{13} \mathrm{C}$ NMR spectrum of compound 10 in $\mathrm{CDCl}_{3}$ at $125 \mathrm{MHz}$.

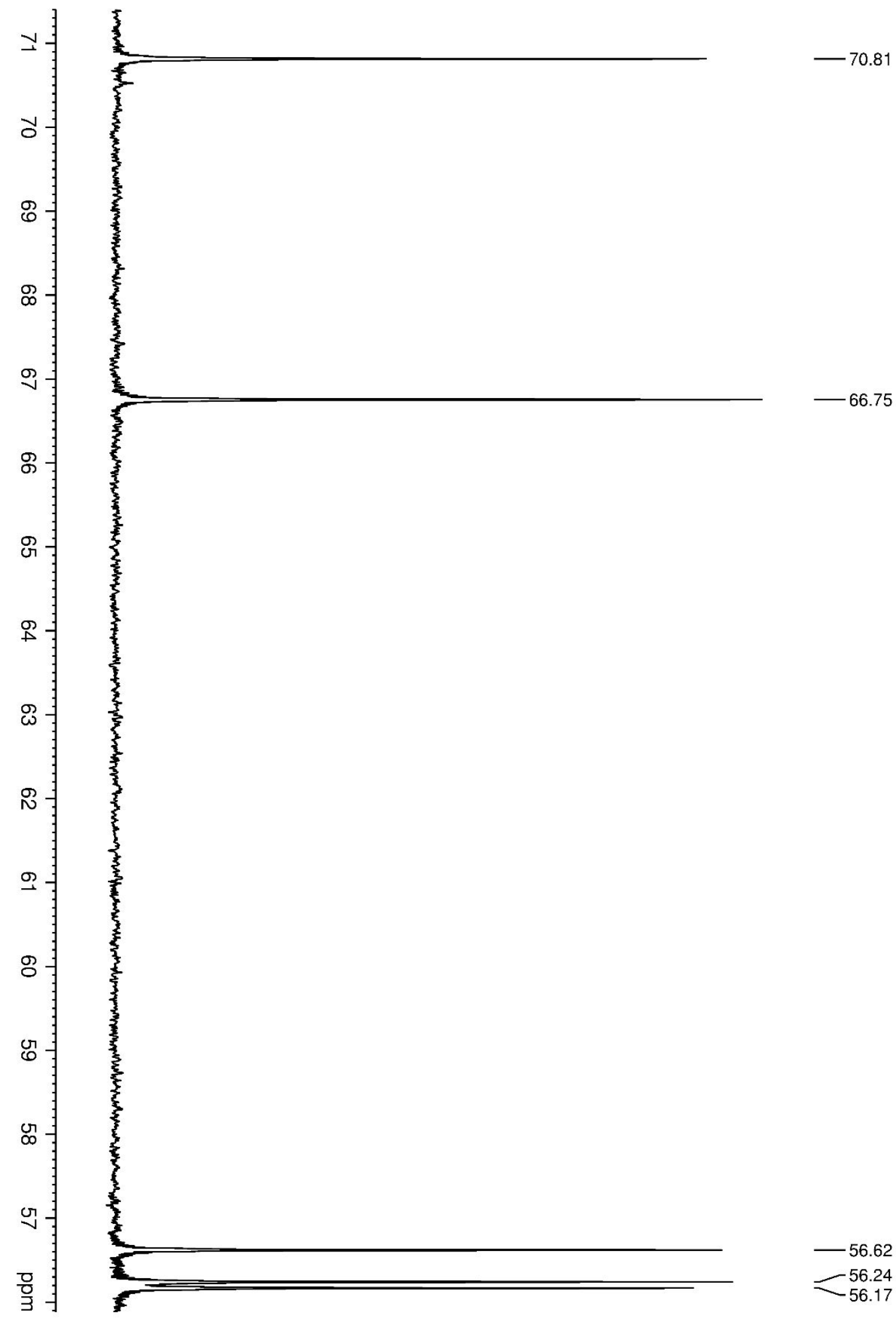


Figure S105. ${ }^{13} \mathrm{C}$ NMR DEP135 spectrum of compound 10 in $\mathrm{CDCl}_{3}$ at $125 \mathrm{MHz}$.

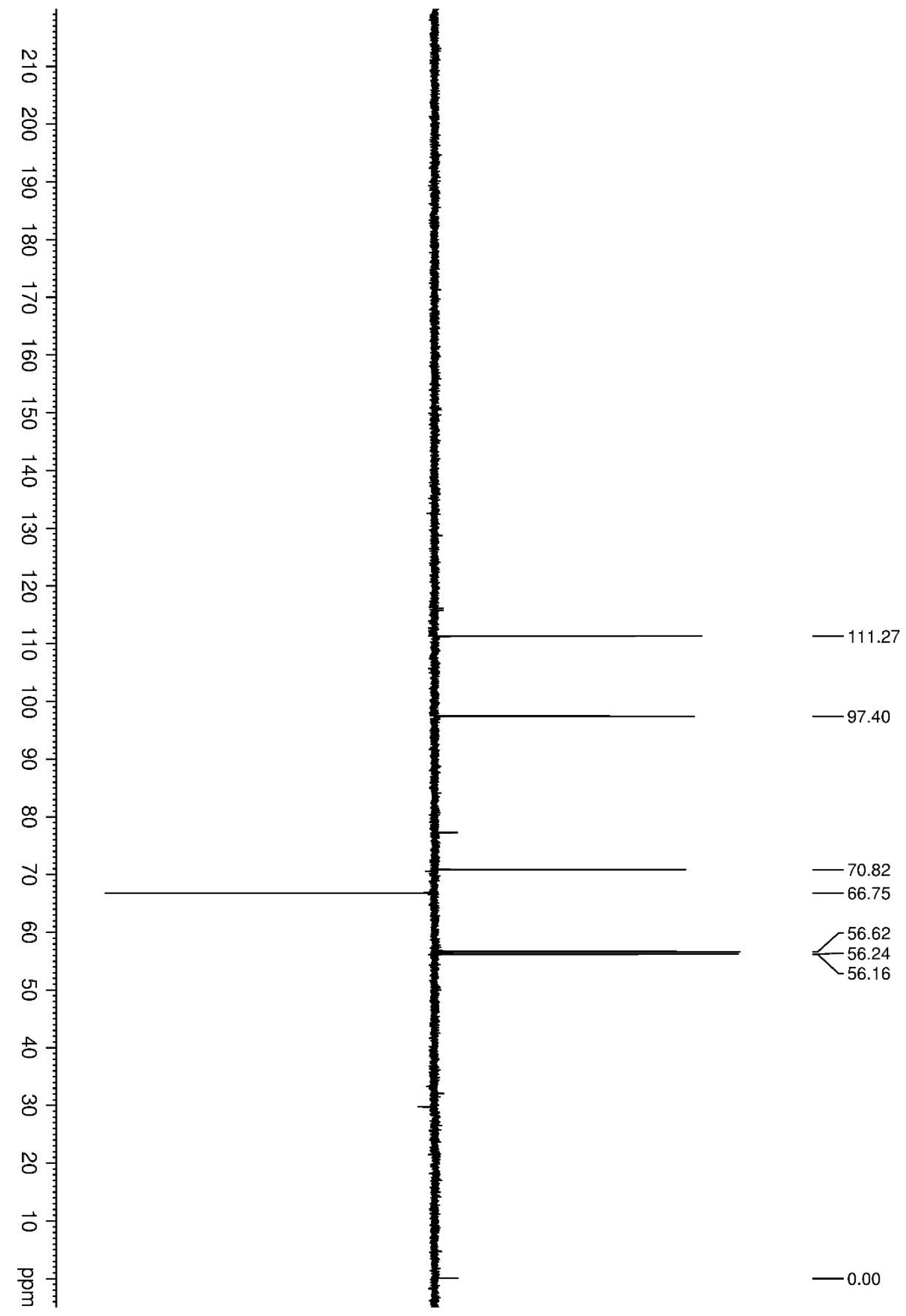


Figure S106. Enlargement ( $\delta$ 111.2-56.1) of ${ }^{13} \mathrm{C}$ NMR DEP135 spectrum of compound 10 in $\mathrm{CDCl}_{3}$ at $125 \mathrm{MHz}$.

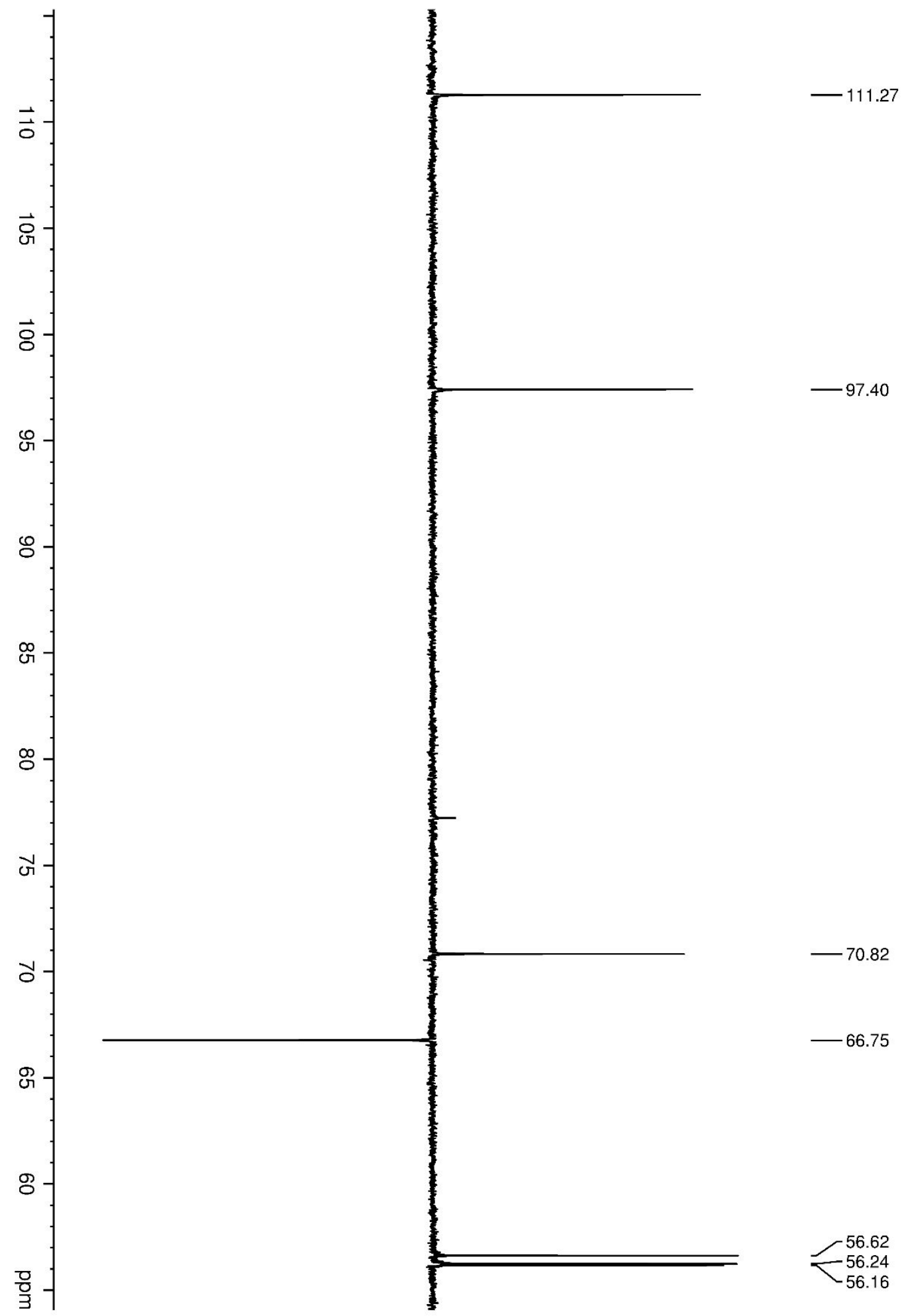


Figure S107. Enlargement $(\delta 70.8-56.1)$ of ${ }^{13} \mathrm{C}$ NMR DEP135 spectrum of compound 10 in $\mathrm{CDCl}_{3}$ at $125 \mathrm{MHz}$.

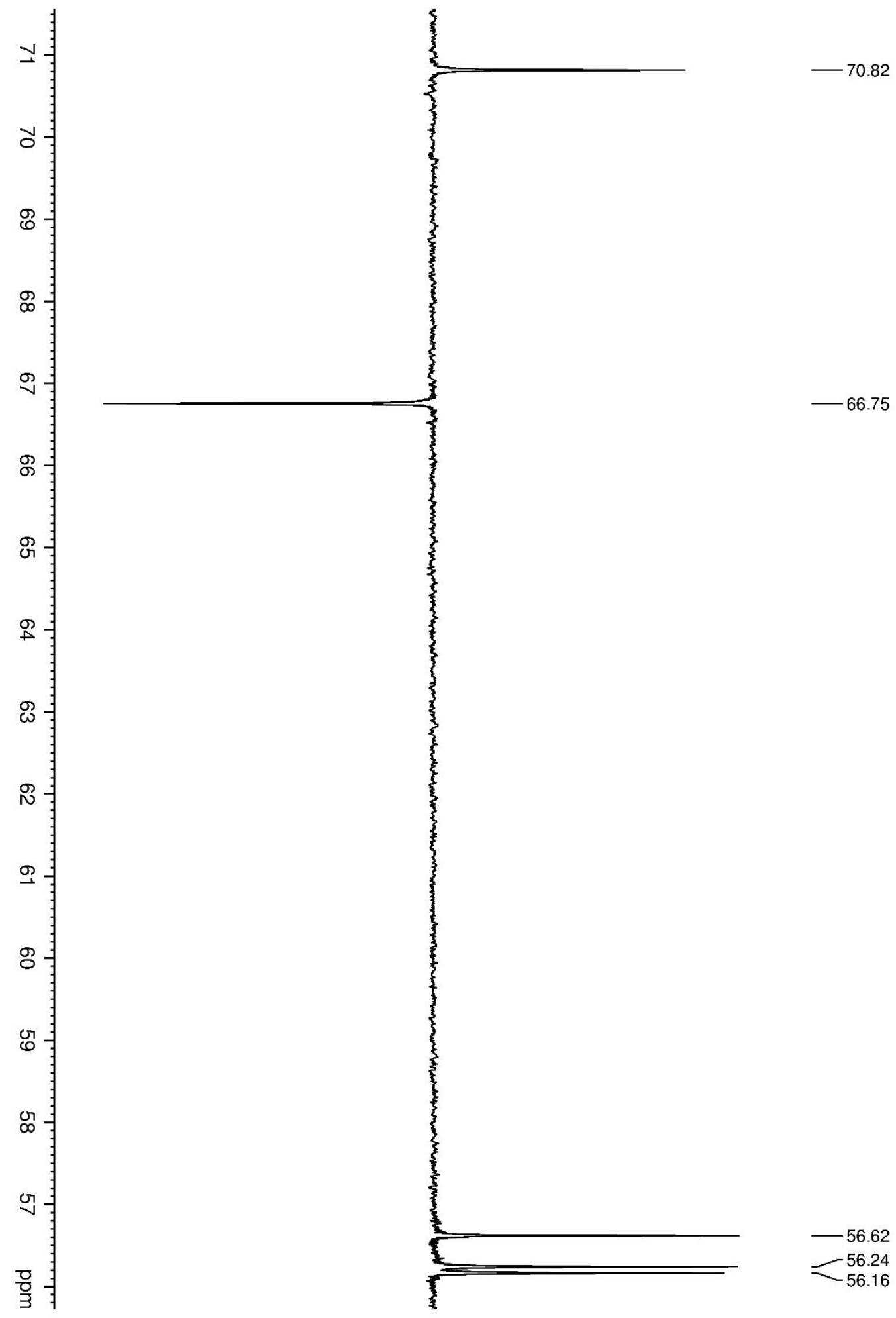


Figure S108. ${ }^{1} \mathrm{H}-{ }^{1} \mathrm{H}$ COSY correlation map from ${ }^{1} \mathrm{H}$ NMR experiment of compound 10 in $\mathrm{CDCl}_{3}$ at $500 \mathrm{MHz}$.

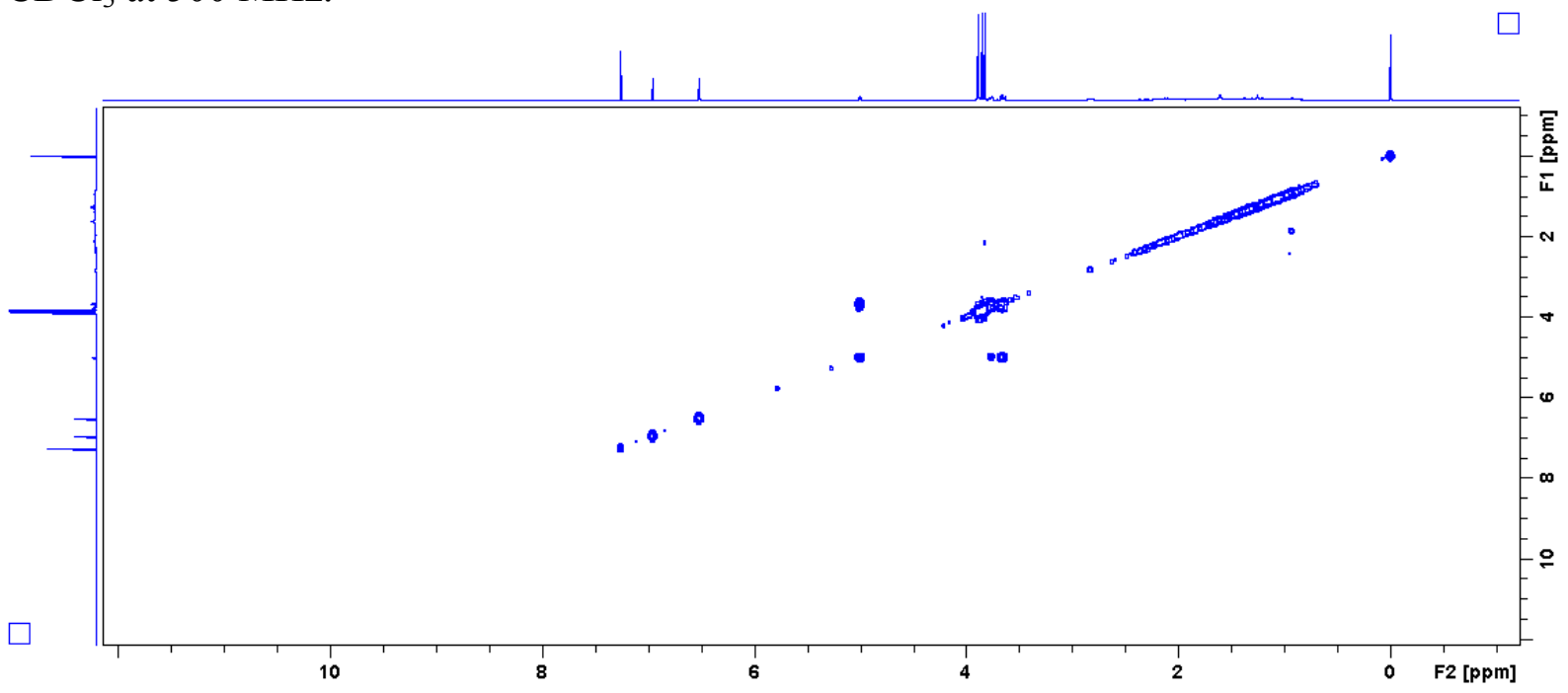

Figure S109. Enlargement $\left(\delta\right.$ 7.26-3.63) of ${ }^{1} \mathrm{H}-{ }^{1} \mathrm{H}$ COSY correlation map from ${ }^{1} \mathrm{H}$ NMR experiment of compound 10 in $\mathrm{CDCl}_{3}$ at $500 \mathrm{MHz}$.

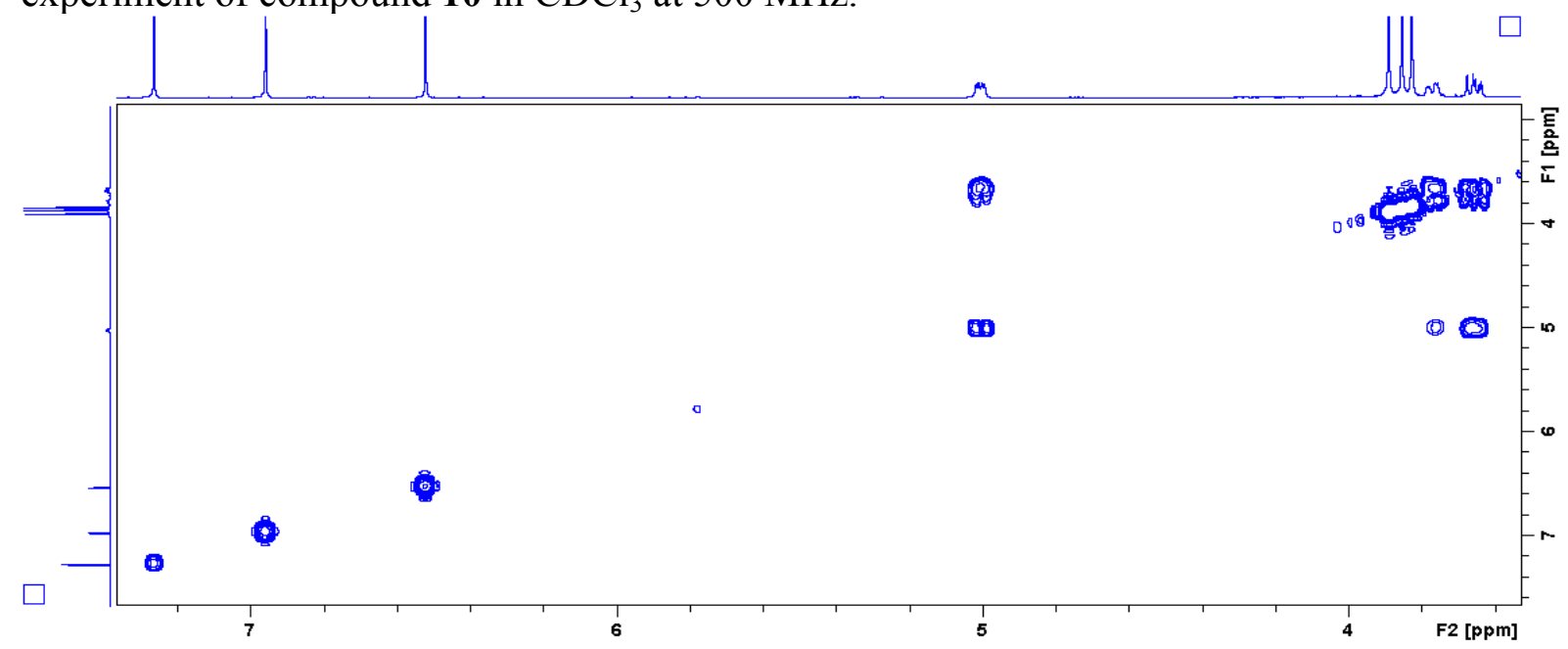

Figure S110. Enlargement $\left(\delta\right.$ 5.01-3.63) of ${ }^{1} \mathrm{H}-{ }^{1} \mathrm{H}$ COSY correlation map from ${ }^{1} \mathrm{H}$ NMR experiment of compound $\mathbf{1 0}$ in $\mathrm{CDCl}_{3}$ at $500 \mathrm{MHz}$.

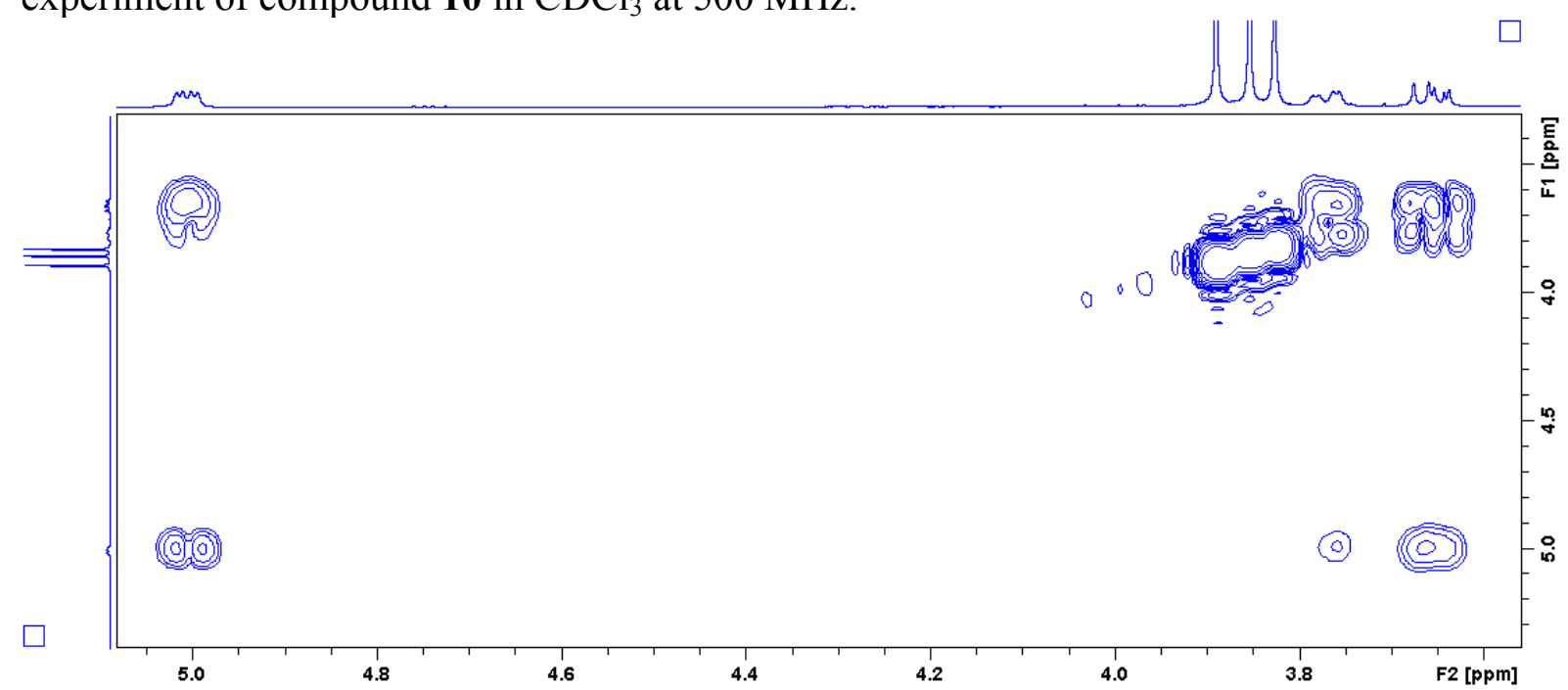


Figure S111. ${ }^{1} \mathrm{H}-{ }^{13} \mathrm{C}$ one-bond correlation map from HSQC NMR experiment of compound $\mathbf{1 0}$ in $\mathrm{CDCl}_{3}$ at 500 and $125 \mathrm{MHz}$.

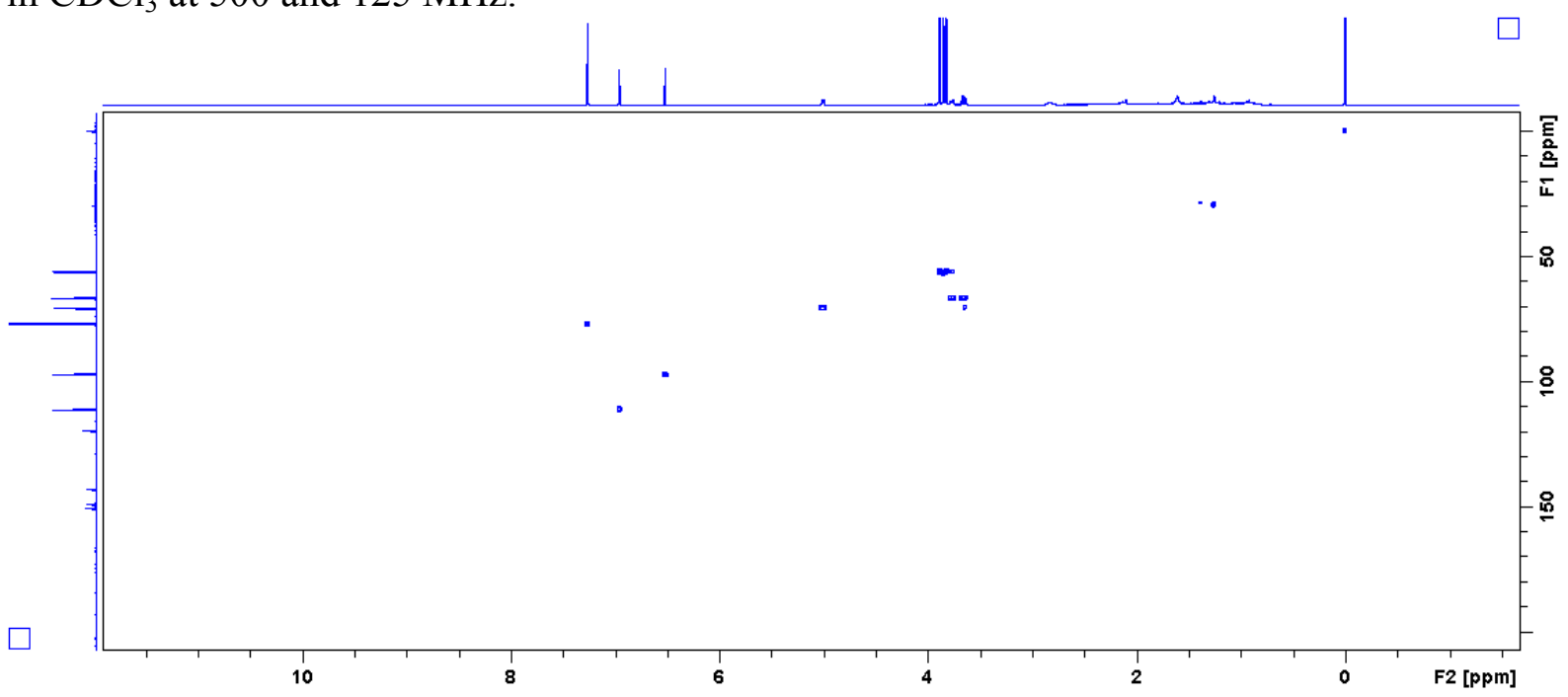

Figure S112. Enlargement $(\delta 8.23-6.09)$ of ${ }^{1} \mathrm{H}-{ }^{13} \mathrm{C}$ one-bond correlation map from HSQC NMR experiment of compound $\mathbf{1 0}$ in $\mathrm{CDCl}_{3}$ at 500 and $125 \mathrm{MHz}$.

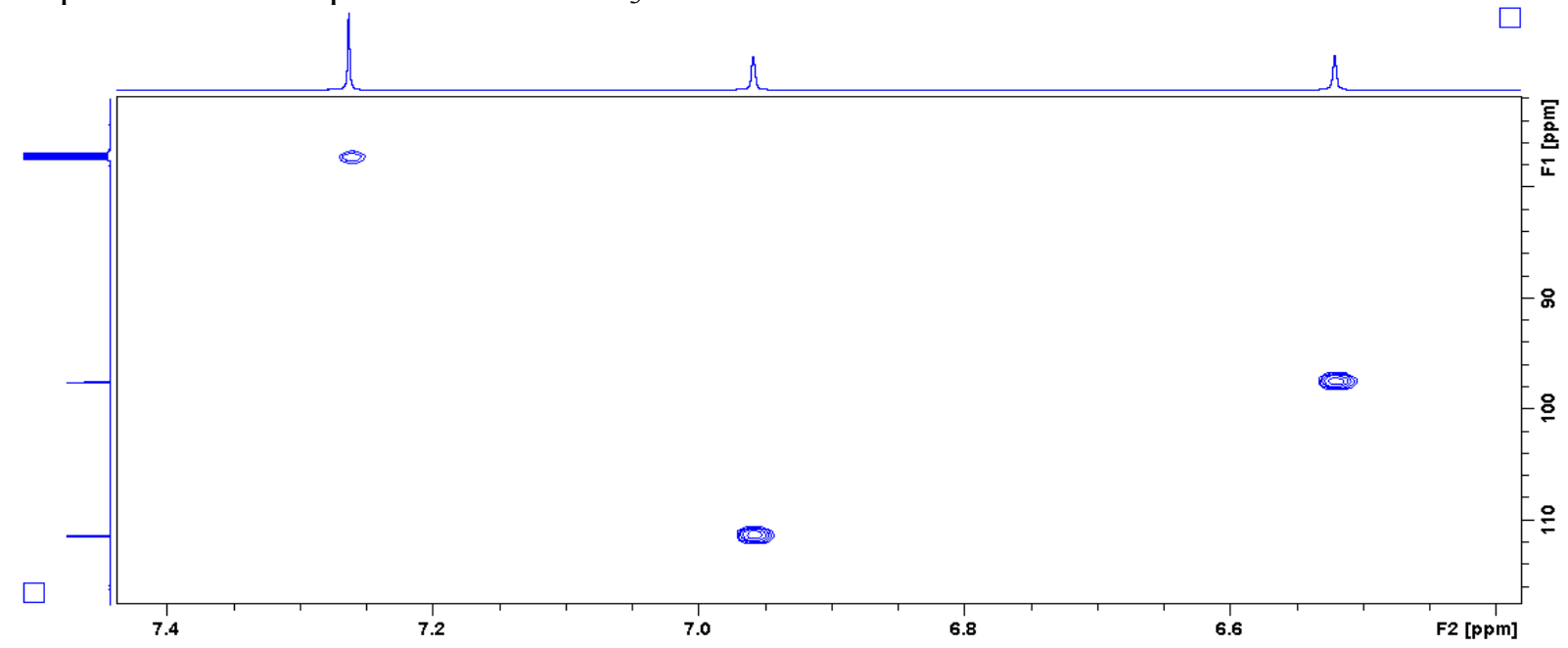

Figure S113. Enlargement $(\delta 4.02-3.08)$ of ${ }^{1} \mathrm{H}-{ }^{13} \mathrm{C}$ one-bond correlation map from HSQC NMR experiment of compound $\mathbf{1 0}$ in $\mathrm{CDCl}_{3}$ at 500 and $125 \mathrm{MHz}$.

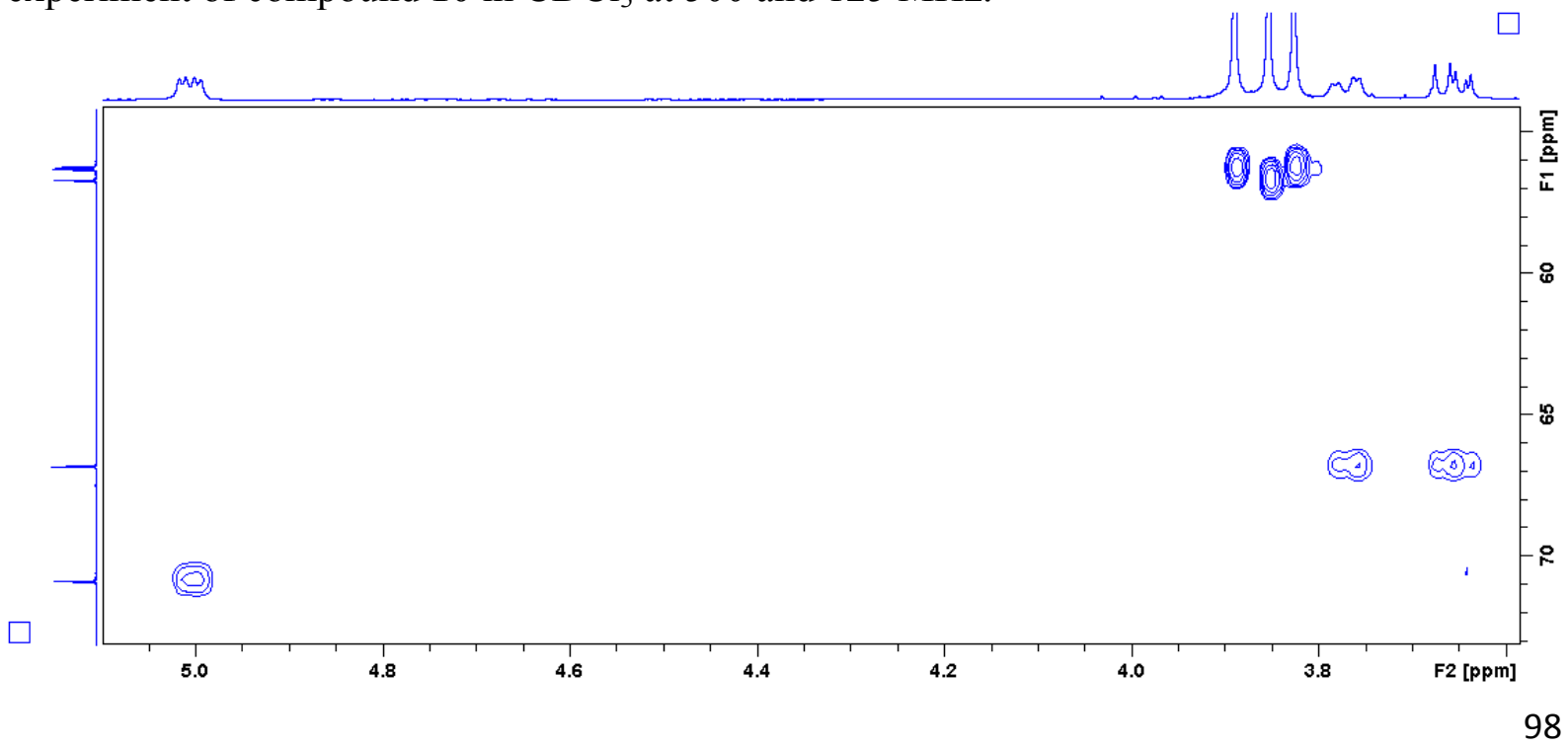


Figure S114. ${ }^{1} \mathrm{H}-{ }^{13} \mathrm{C}$ long-range correlation map from HMBC NMR experiment of compound 10 in $\mathrm{CDCl}_{3}$ at 500 and $125 \mathrm{MHz}$.

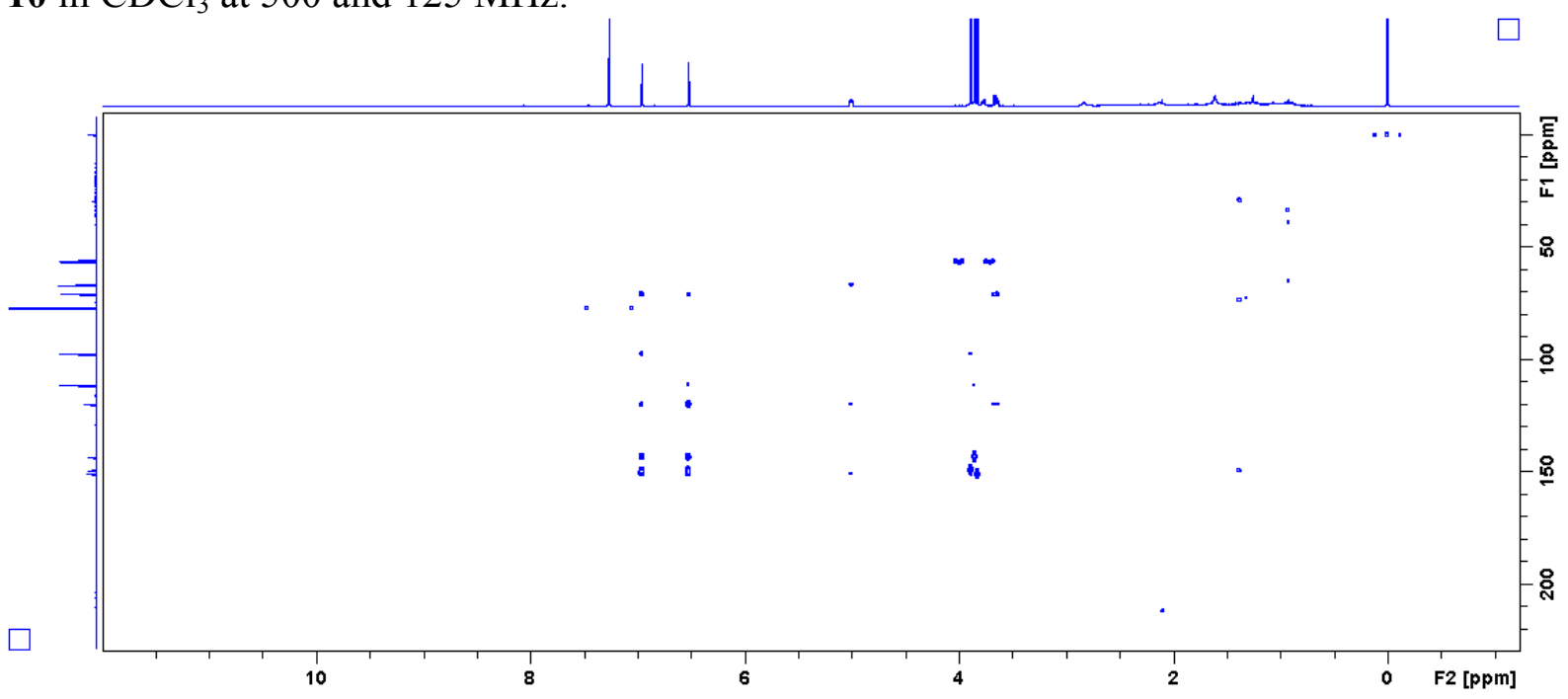

Figure S115. Enlargement $(\delta 7.45-6.50)$ of ${ }^{1} \mathrm{H}-{ }^{13} \mathrm{C}$ long-range correlation map from HMBC NMR experiment of compound 10 in $\mathrm{CDCl}_{3}$ at 500 and $125 \mathrm{MHz}$.

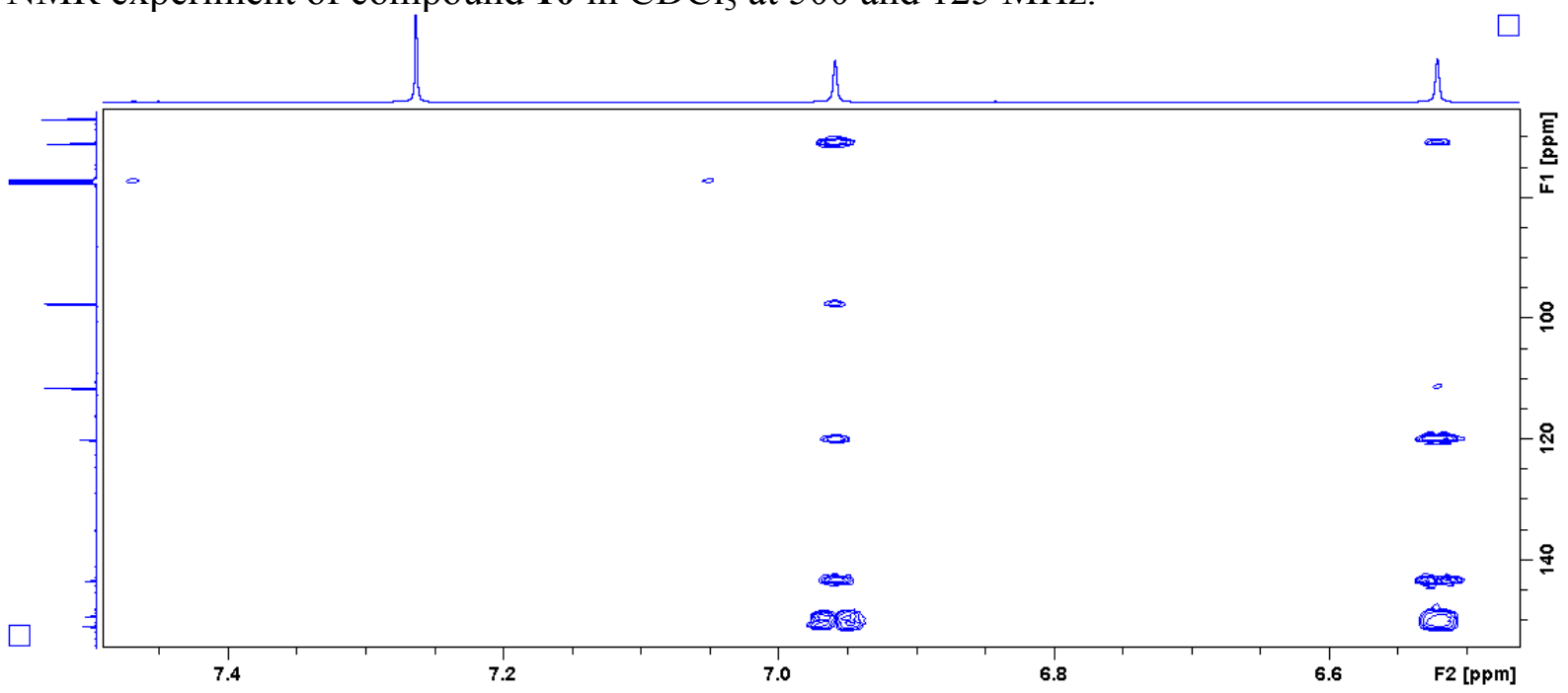

Figure S116. Enlargement $(\delta 5.00-3.60)$ of ${ }^{1} \mathrm{H}-{ }^{13} \mathrm{C}$ long-range correlation map from HMBC NMR experiment of compound $\mathbf{1 0}$ in $\mathrm{CDCl}_{3}$ at 500 and $125 \mathrm{MHz}$.

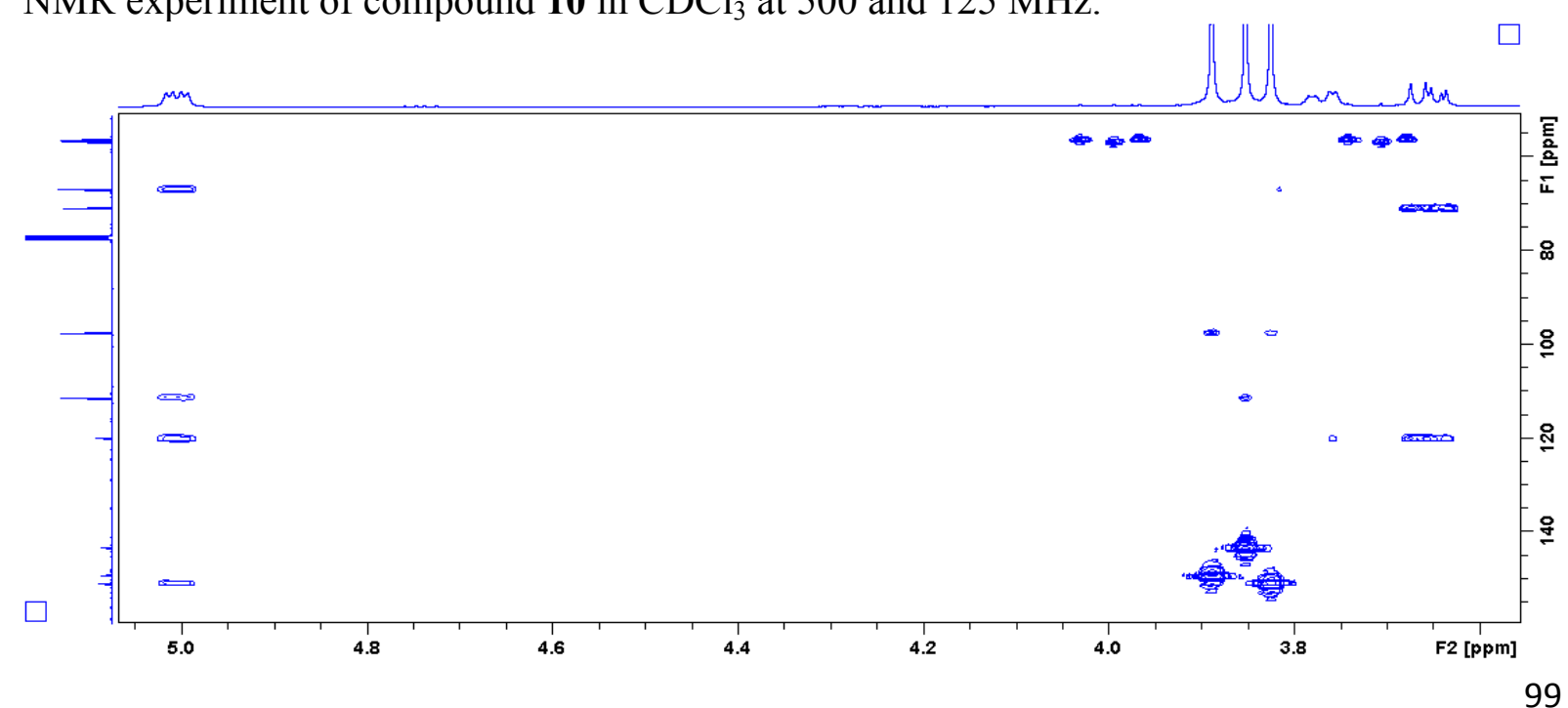


Figure S117. HRESIMS spectrum of compound $\mathbf{1 0 .}$

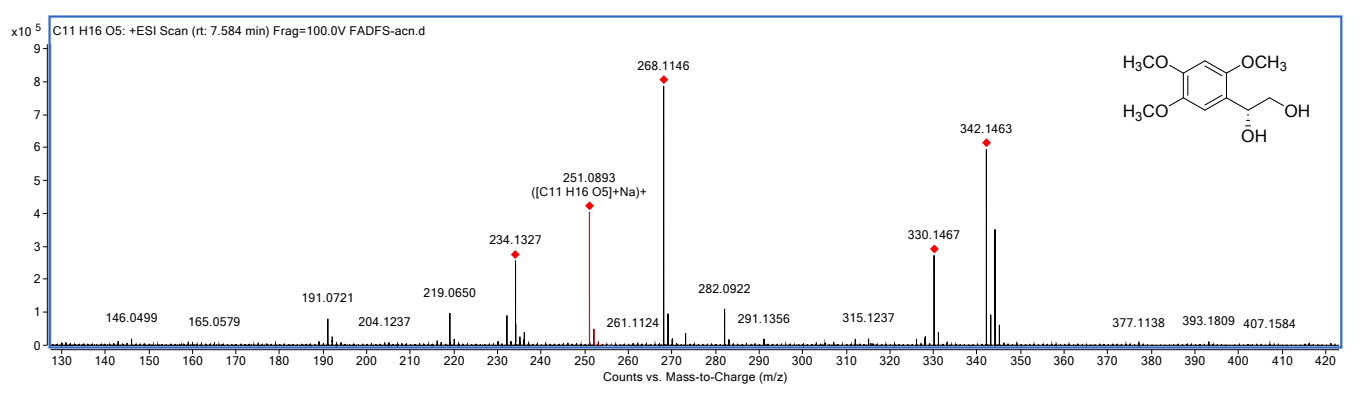


Figure S118. Structures of alkaloids 2, 6, 7, 9 and 11 whose NMR data were fully assigned.
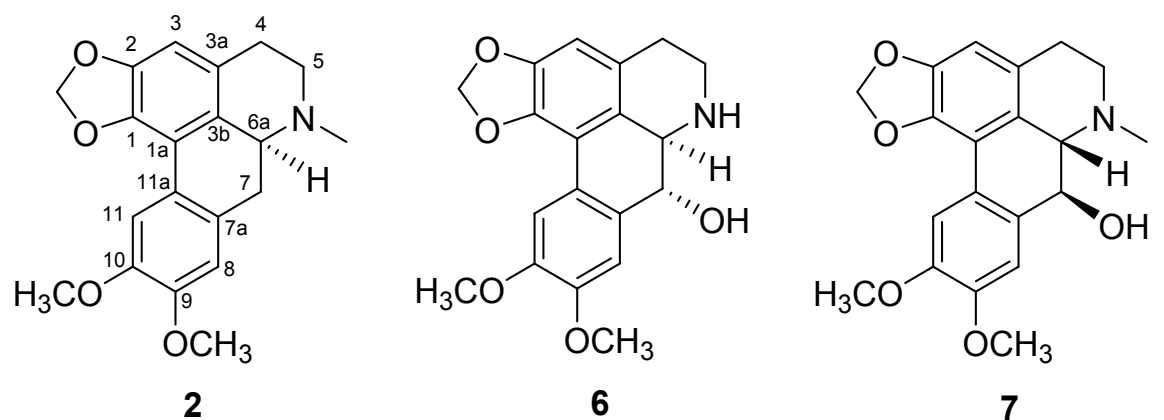<smiles>COc1cc2c(cc1OC)-c1c3c(cc4ccnc(c14)C2=O)OCO3</smiles>

9<smiles>COc1cc2c(cc1OC)-c1c3c(cc4c1[C@@H](O)[C@](C)(CC2)[N+]4(C)C)OCO3</smiles>

11 
Table S1. ${ }^{1} \mathrm{H}$ NMR Data for Compounds 2,6,7 and 9 (500 MHz), and 11 (300 MHz) ( $\delta$ in ppm and $J$ Values in (Hz) in Parentheses)

\begin{tabular}{|c|c|c|c|c|c|}
\hline position & $\mathbf{2}^{a}$ & $\mathbf{6}^{a}$ & $7^{a}$ & $9^{b}$ & $11^{b}$ \\
\hline \multicolumn{6}{|l|}{1} \\
\hline \multicolumn{6}{|l|}{$1 \mathrm{a}$} \\
\hline \multicolumn{6}{|l|}{2} \\
\hline 3 & $6.52 \mathrm{~s}$ & $6.52 \mathrm{~s}$ & $6.53 \mathrm{~s}$ & $7.11 \mathrm{~s}$ & $6.55 \mathrm{~s}$ \\
\hline \multicolumn{6}{|l|}{$3 a$} \\
\hline \multicolumn{6}{|l|}{$3 b$} \\
\hline $4 a x$ & $2.64 \mathrm{~m}$ & $2.77 \mathrm{~m}$ & $2.61 \mathrm{dt}(5.2 ; 16.3)$ & $7.76 \mathrm{~d}(5.2)$ & $3.01 \mathrm{dd}(4.8 ; 18.0)$ \\
\hline 4 eq & $3.15 \mathrm{~m}$ & $3.25 \mathrm{~m}$ & 2.81 ddd $(4.6 ; 7.6 ; 16.3)$ & & 3.21 ddd $(6.5 ; 13.0 ; 18.0)$ \\
\hline $5 \mathrm{ax}$ & $2.58 \mathrm{~m}$ & $3.10 \mathrm{~m}$ & $3.02 \mathrm{~m}$ & $8.79 \mathrm{~d}(5.2)$ & 3.66 ddd $(6.5 ; 13.0 ; 18.6)$ \\
\hline $5 e q$ & $3.09 \mathrm{~m}$ & $3.67 \mathrm{~m}$ & & & 3.71 ddd $(4.8 ; 13.0 ; 18.6)$ \\
\hline $6 \mathrm{a}$ & $3.23 \mathrm{~m}$ & $4.07 \mathrm{~d}(11.8)$ & $3.52 \mathrm{~d}(12.3)$ & & $4.44 \mathrm{~d}(12.1)$ \\
\hline 7 & $2.69 \mathrm{t}(14.5)$ & $4.91 \mathrm{~d}(11.8)$ & $4.60 \mathrm{dd}(0.7 ; 12.3)$ & & $5.18 \mathrm{~d}(0.9 ; 12.1)$ \\
\hline 1 & $3.09 \mathrm{~m}$ & & & & \\
\hline \multicolumn{6}{|l|}{$7 \mathrm{a}$} \\
\hline 8 & $6.78 \mathrm{~s}$ & $7.27 \mathrm{~s}$ & $7.28 \mathrm{~d}(0.7)$ & $7.91 \mathrm{~s}$ & $7.40 \mathrm{~d}(0.9)$ \\
\hline \multicolumn{6}{|l|}{9} \\
\hline \multicolumn{6}{|l|}{10} \\
\hline 11 & $7.66 \mathrm{~s}$ & $7.58 \mathrm{~s}$ & $7.63 \mathrm{~s}$ & $7.94 \mathrm{~s}$ & $7.62 \mathrm{~s}$ \\
\hline \multicolumn{6}{|l|}{$11 \mathrm{a}$} \\
\hline \multirow{2}{*}{$\left(\mathrm{OCH}_{2} \mathrm{O}\right) 1-2$} & $5.93 \mathrm{~d}(1.4)$ & $5.97 \mathrm{~d}(1.2)$ & $5.95 \mathrm{~d}(1.4)$ & $6.38 \mathrm{~s}$ & $6.00 \mathrm{~d}(1.3)$ \\
\hline & $6.08 \mathrm{~d}(1.4)$ & $6.11 \mathrm{~d}(1.2)$ & $6.07 \mathrm{~d}(1.4)$ & & $6.17(1.3)$ \\
\hline $\mathrm{OCH}_{3}-9$ & $3.92 \mathrm{~s}$ & $3.90 \mathrm{~s}$ & $3.97 \mathrm{~s}$ & $4.06 \mathrm{~s}$ & $3.98 \mathrm{~s}$ \\
\hline $\mathrm{OCH}_{3}-10$ & $3.92 \mathrm{~s}$ & $3.91 \mathrm{~s}$ & $3.93 \mathrm{~s}$ & $4.00 \mathrm{~s}$ & $3.92 \mathrm{~s}$ \\
\hline $\mathrm{N}-\mathrm{CH}_{3}$ & $2.58 \mathrm{~s}$ & & $2.59 \mathrm{~s}$ & & $3.32 \mathrm{~s}$ \\
\hline
\end{tabular}

${ }^{a}$ The experiments were obtained at $293 \mathrm{~K}$ with TMS as internal reference $(0.00 \mathrm{ppm})$ in $\mathrm{CDCl}_{3} \cdot{ }^{\mathrm{b}}$ in $\mathrm{CDCl}_{3}+\mathrm{drops}_{\mathrm{C}} \mathrm{CD}_{3} \mathrm{OD}$. 
Table S2. ${ }^{13} \mathrm{C}$ NMR Data for Compounds 2,6,7 and $9(125 \mathrm{MHz})$, and $11(75 \mathrm{MHz})(\delta$ in ppm)

\begin{tabular}{llllll}
\hline position & $\mathbf{2}^{\boldsymbol{a}}$ & $\mathbf{6}^{\boldsymbol{a}}$ & $\mathbf{7}^{\boldsymbol{a}}$ & $\mathbf{9}^{\boldsymbol{b}}$ & $\mathbf{1 1}^{\boldsymbol{a}}$ \\
\hline 1 & 142.0 & 142.2 & 141.9 & 147.0 & 142.9 \\
$1 \mathrm{a}$ & 116.6 & 115.9 & 116.8 & 108.2 & 116.0 \\
2 & 147.1 & 148.0 & 147.0 & 151.5 & 148.8 \\
3 & 106.6 & 106.9 & 106.8 & 102.7 & 106.4 \\
$3 \mathrm{a}$ & 118.2 & 119.8 & 127.8 & 135.4 & 123.0 \\
$3 \mathrm{~b}$ & 125.8 & 125.4 & 122.7 & 122.5 & 119.1 \\
4 & 28.6 & 26.7 & 22.4 & 124.1 & 27.2 \\
5 & 53.3 & 41.5 & 48.7 & 144.7 & 67.1 \\
$6 \mathrm{a}$ & 62.1 & 59.2 & 65.1 & 145.3 & 76.6 \\
7 & 33.8 & 70.1 & 69.5 & 181.0 & 68.5 \\
$7 \mathrm{a}$ & 127.9 & 130.4 & 132.0 & 125.8 & 130.7 \\
8 & 111.2 & 107.5 & 107.3 & 109.6 & 107.5 \\
9 & 148.7 & 149.1 & 148.9 & 149.4 & 149.6 \\
10 & 147.6 & 148.2 & 147.8 & 153.7 & 148.1 \\
11 & 110.5 & 110.3 & 110.4 & 108.8 & 110.0 \\
$11 \mathrm{a}$ & 123.4 & 121.2 & 121.1 & 127.6 & 119.6 \\
$\left(\mathrm{OCH}_{2} \mathrm{O}\right) 1-2$ & 100.6 & 101.1 & 100.7 & 102.5 & 101.4 \\
$\mathrm{OCH}_{3}-9$ & 55.9 & 55.9 & 55.9 & 56.3 & 56.0 \\
$\mathrm{OCH}_{3}-10$ & 55.9 & 56.1 & 56.1 & 56.1 & 56.1 \\
$\mathrm{~N}-\mathrm{CH}_{3}$ & 43.4 & & 40.6 & & 48.9 \\
\hline
\end{tabular}

${ }^{a}$ The experiments were obtained at $293 \mathrm{~K}$ with TMS as internal reference $(0.00 \mathrm{ppm})$ in $\mathrm{CDCl}_{3}$. ${ }^{\mathrm{b}}$ in $\mathrm{CDCl}_{3}+$ drops of $\mathrm{CD}_{3} \mathrm{OD}$. 
Figure S119. ${ }^{1} \mathrm{H}$ NMR spectrum of compound 1 in $\mathrm{CDCl}_{3}$ at $500 \mathrm{MHz}$.

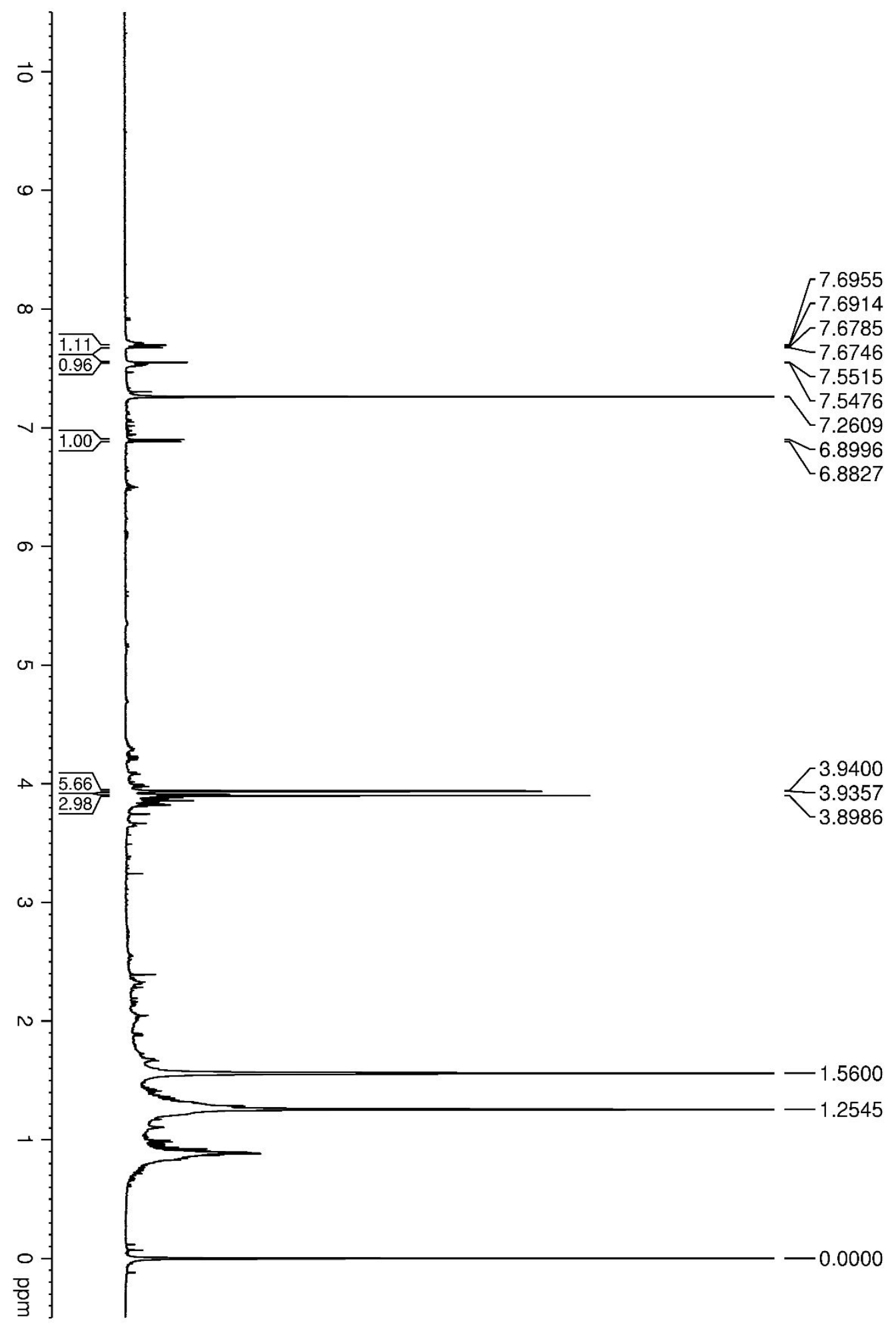


Figure S120. Enlargement $\left(\delta\right.$ 7.69-6.88) of ${ }^{1} \mathrm{H}$ NMR spectrum of compound 1 in $\mathrm{CDCl}_{3}$ at $500 \mathrm{MHz}$.

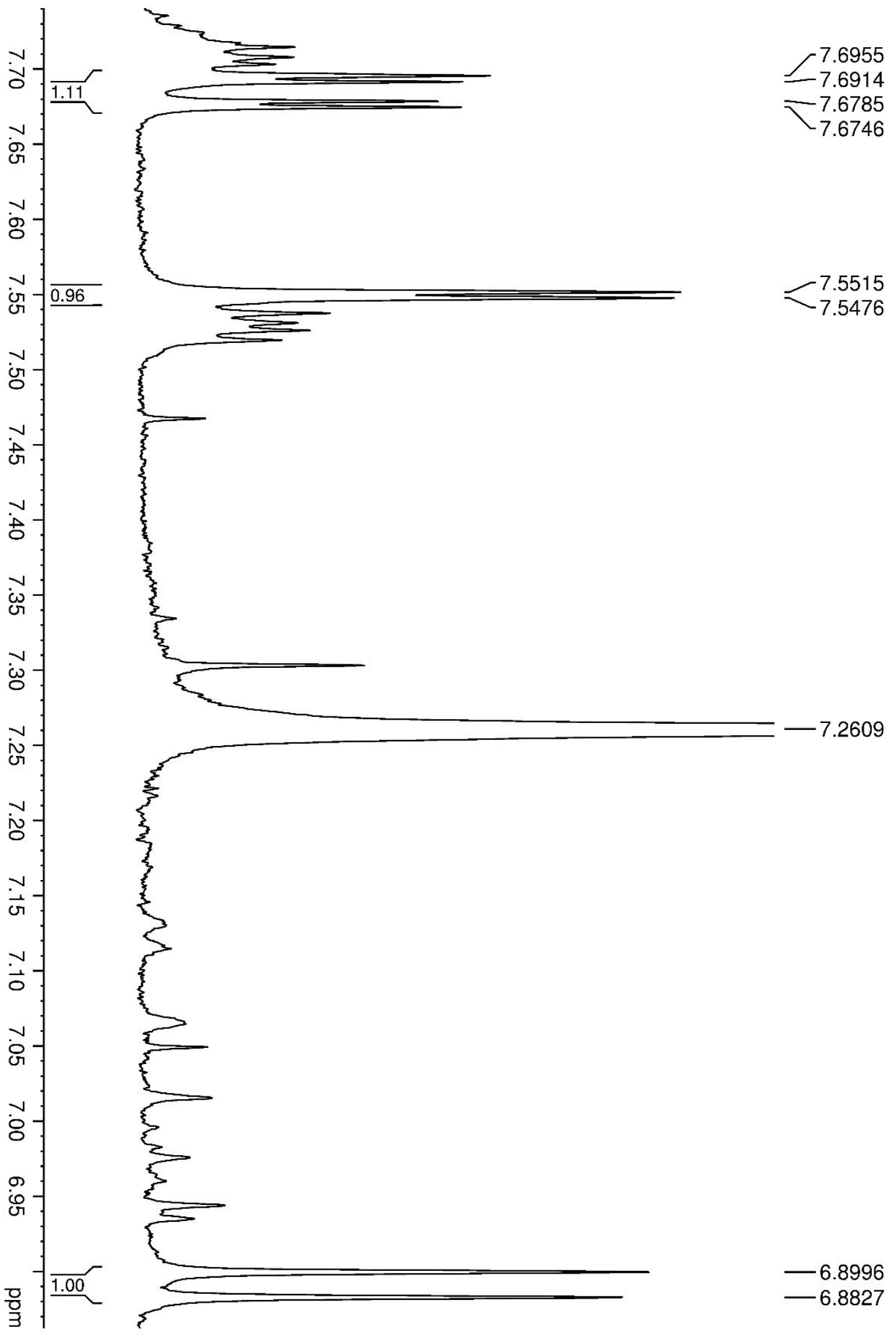


Figure S121. Enlargement ( $\delta$ 3.94-3.89) of ${ }^{1} \mathrm{H}$ NMR spectrum of compound 1 in $\mathrm{CDCl}_{3}$ at $500 \mathrm{MHz}$.

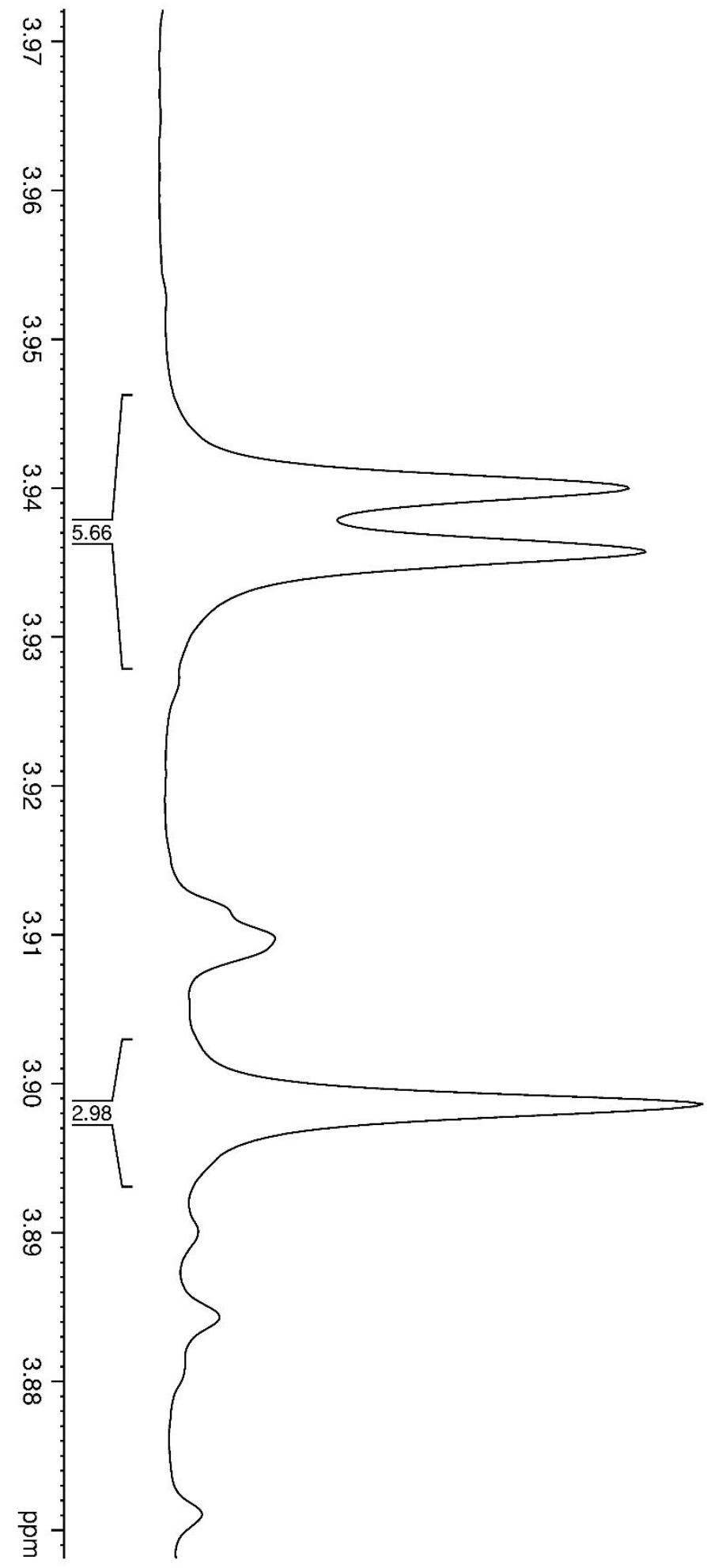


Figure S122. ${ }^{1} \mathrm{H}$ NMR spectrum of compound 2 in $\mathrm{CDCl}_{3}$ at $500 \mathrm{MHz}$.

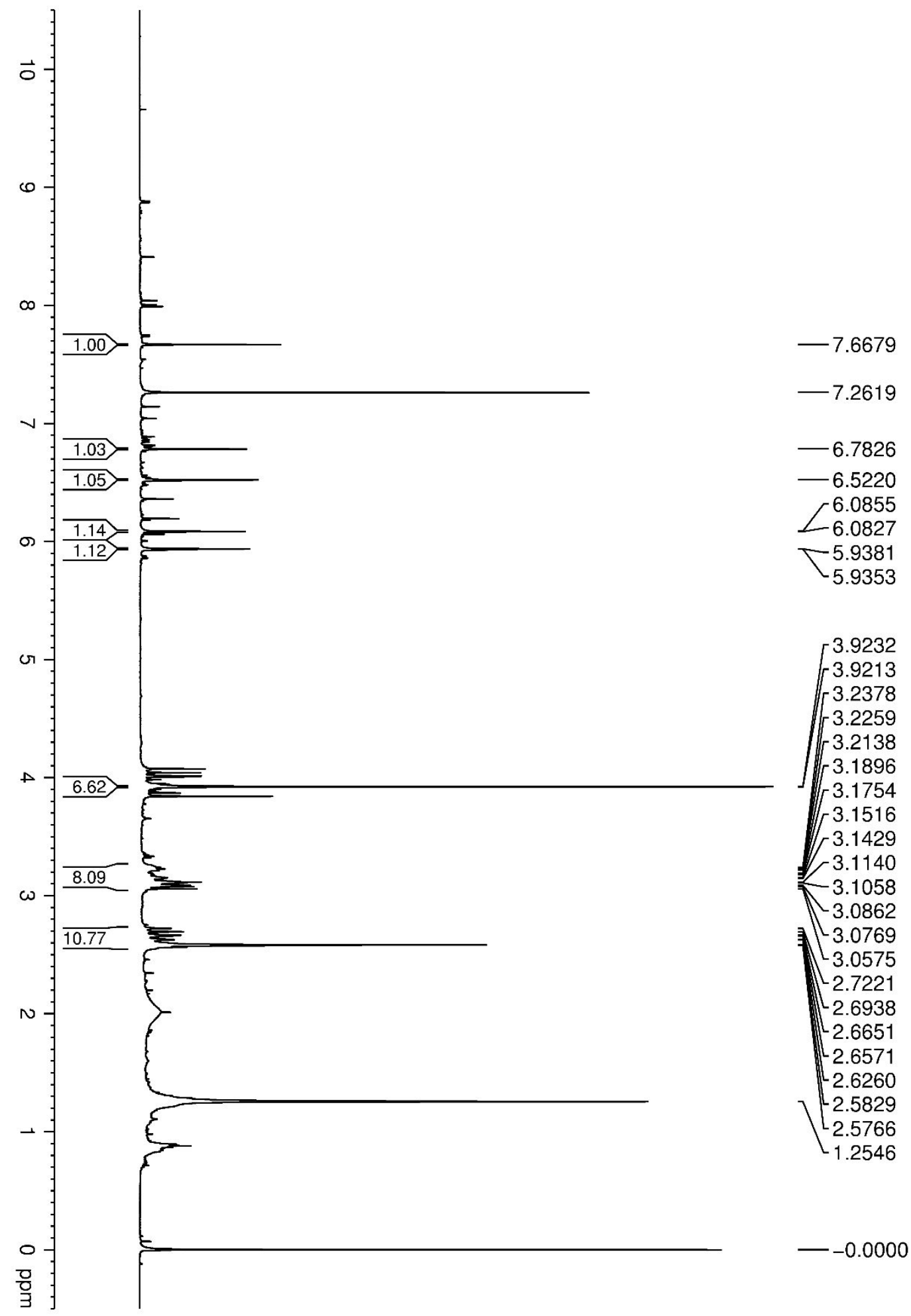


Figure S123. Enlargement $\left(\delta\right.$ 7.66-6.52) of ${ }^{1} \mathrm{H}$ NMR spectrum of compound 2 in $\mathrm{CDCl}_{3}$ at $500 \mathrm{MHz}$.

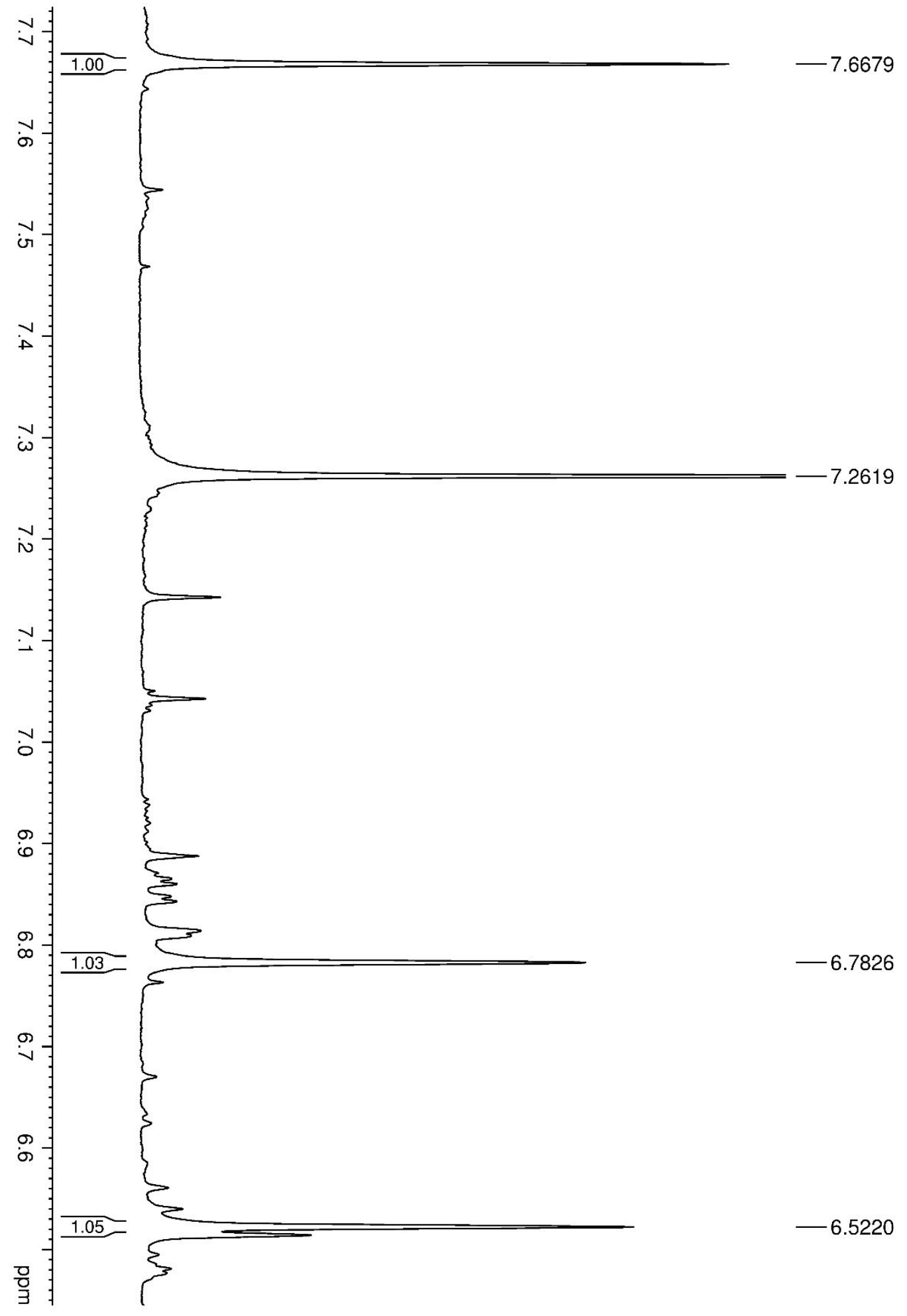


Figure S124. Enlargement $(\delta 6.08-5.93)$ of ${ }^{1} \mathrm{H}$ NMR spectrum of compound 2 in $\mathrm{CDCl}_{3}$ at $500 \mathrm{MHz}$.

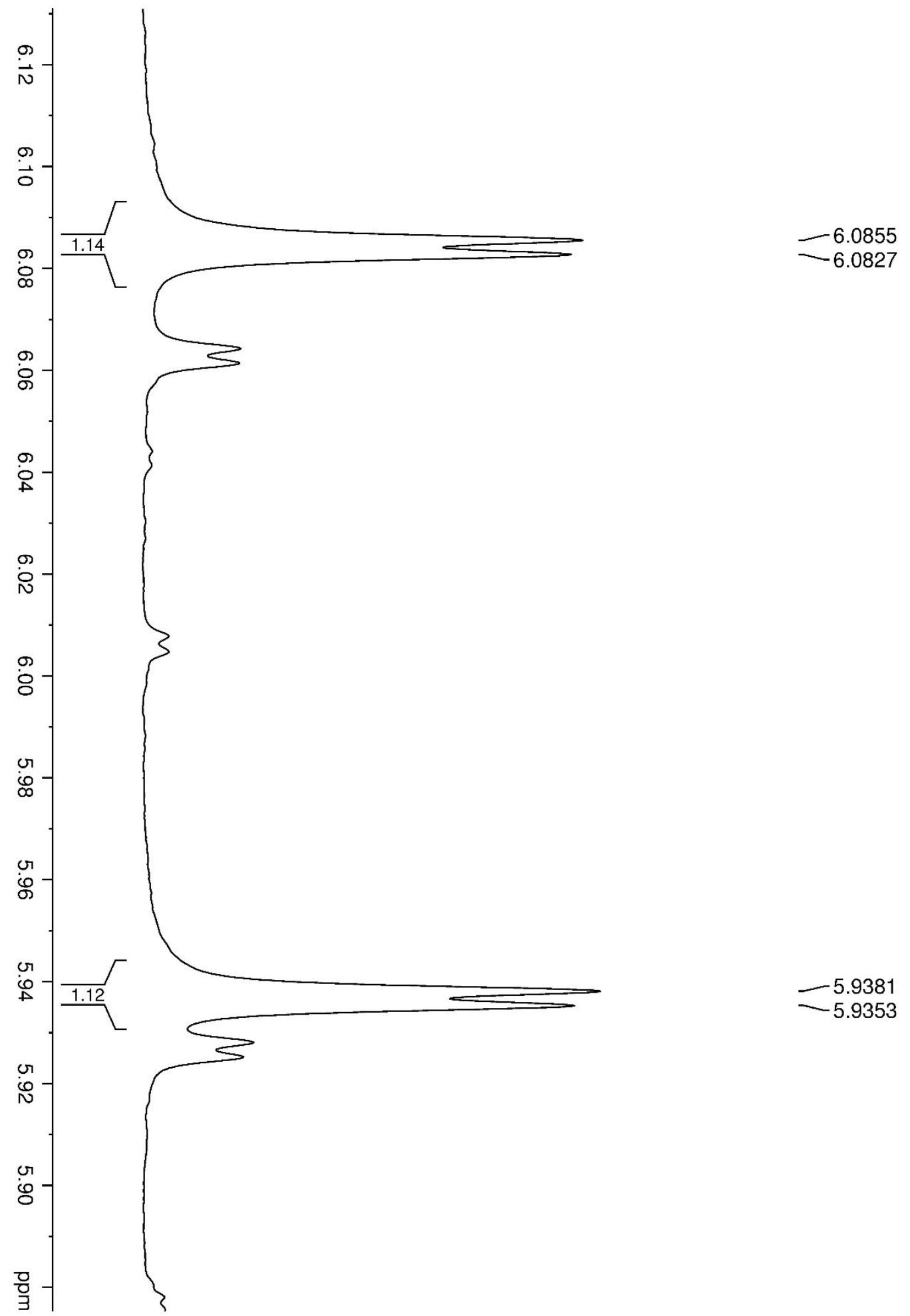


Figure S125. Enlargement $(\delta 3.92-2.57)$ of ${ }^{1} \mathrm{H}$ NMR spectrum of compound 2 in $\mathrm{CDCl}_{3}$ at $500 \mathrm{MHz}$.

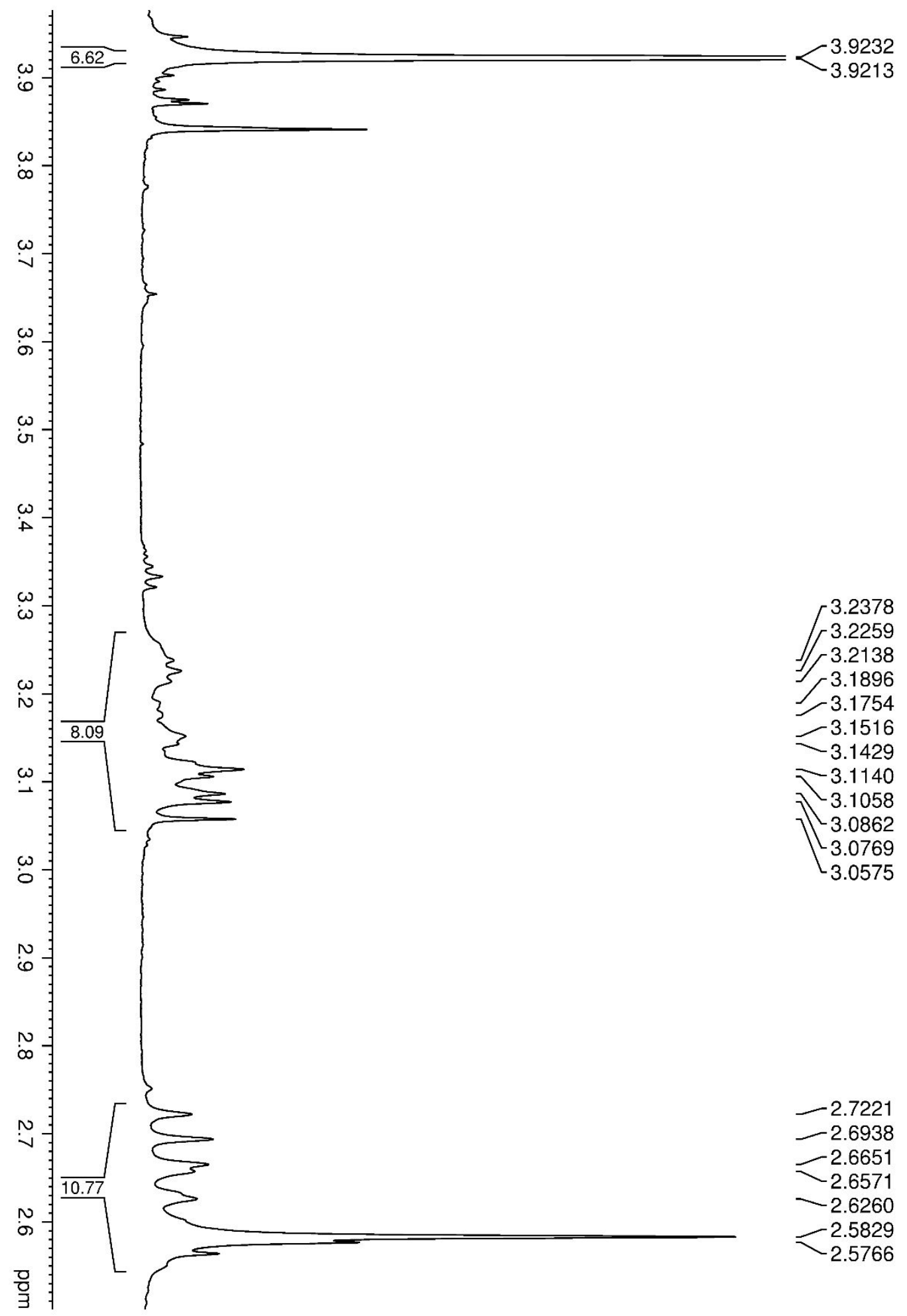


Figure S126. ${ }^{1} \mathrm{H}$ NMR spectrum of compound 6 in $\mathrm{CDCl}_{3}$ at $500 \mathrm{MHz}$.

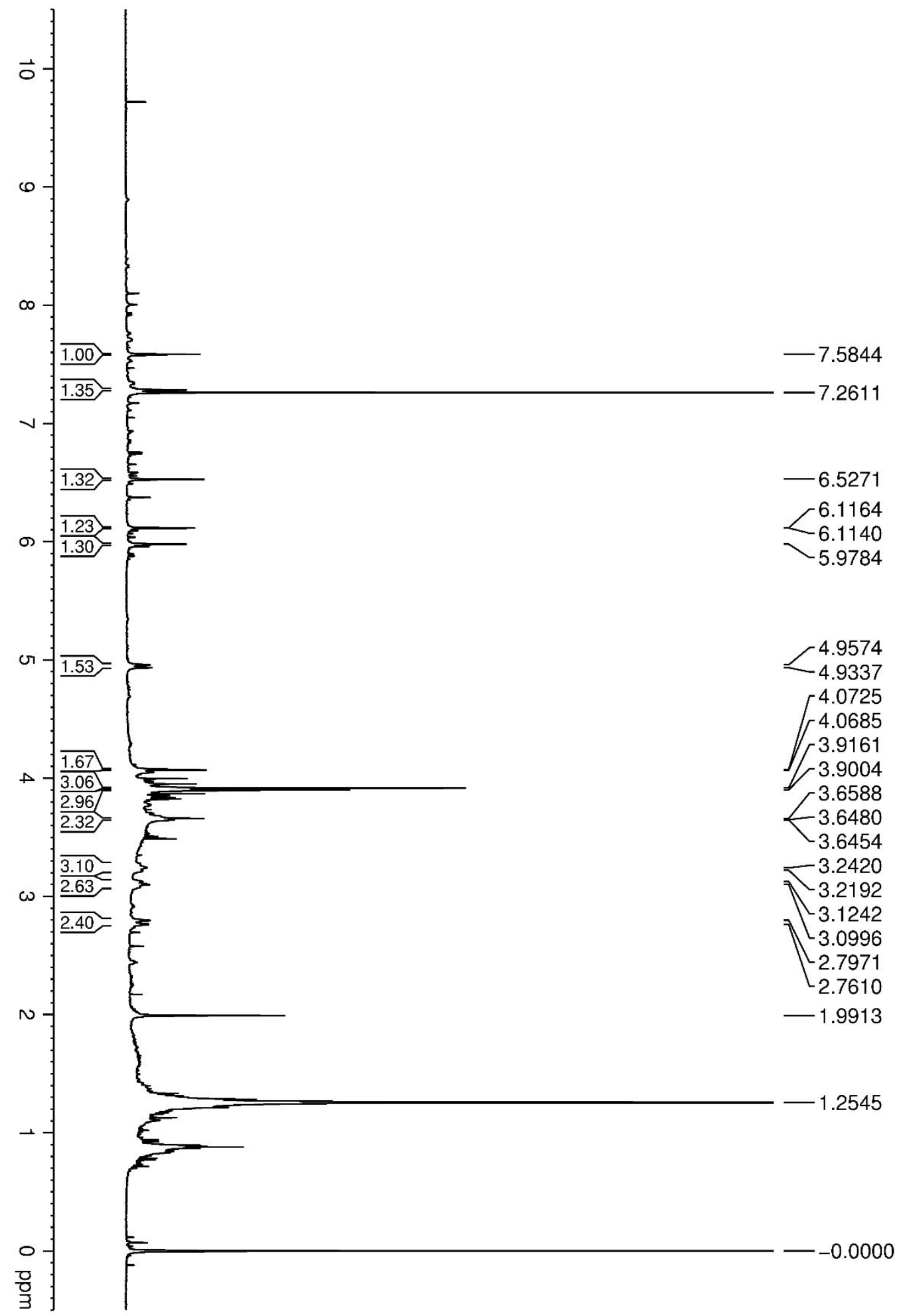


Figure S127. Enlargement $(\delta 7.58-6.52)$ of ${ }^{1} \mathrm{H}$ NMR spectrum of compound 6 in $\mathrm{CDCl}_{3}$ at $500 \mathrm{MHz}$.

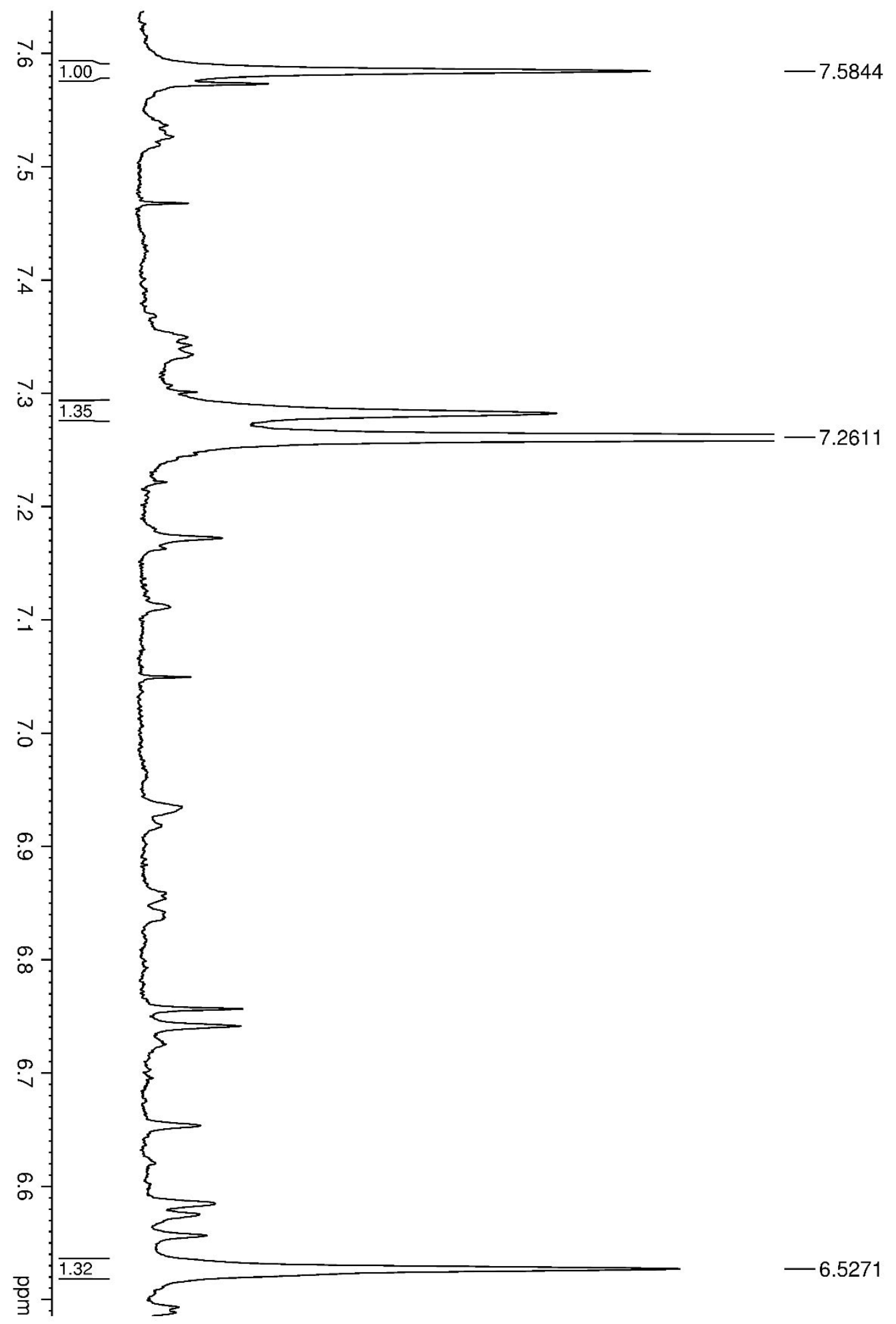


Figure S128. Enlargement ( $\delta 6.11-4.93)$ of ${ }^{1} \mathrm{H}$ NMR spectrum of compound 6 in $\mathrm{CDCl}_{3}$ at $500 \mathrm{MHz}$.

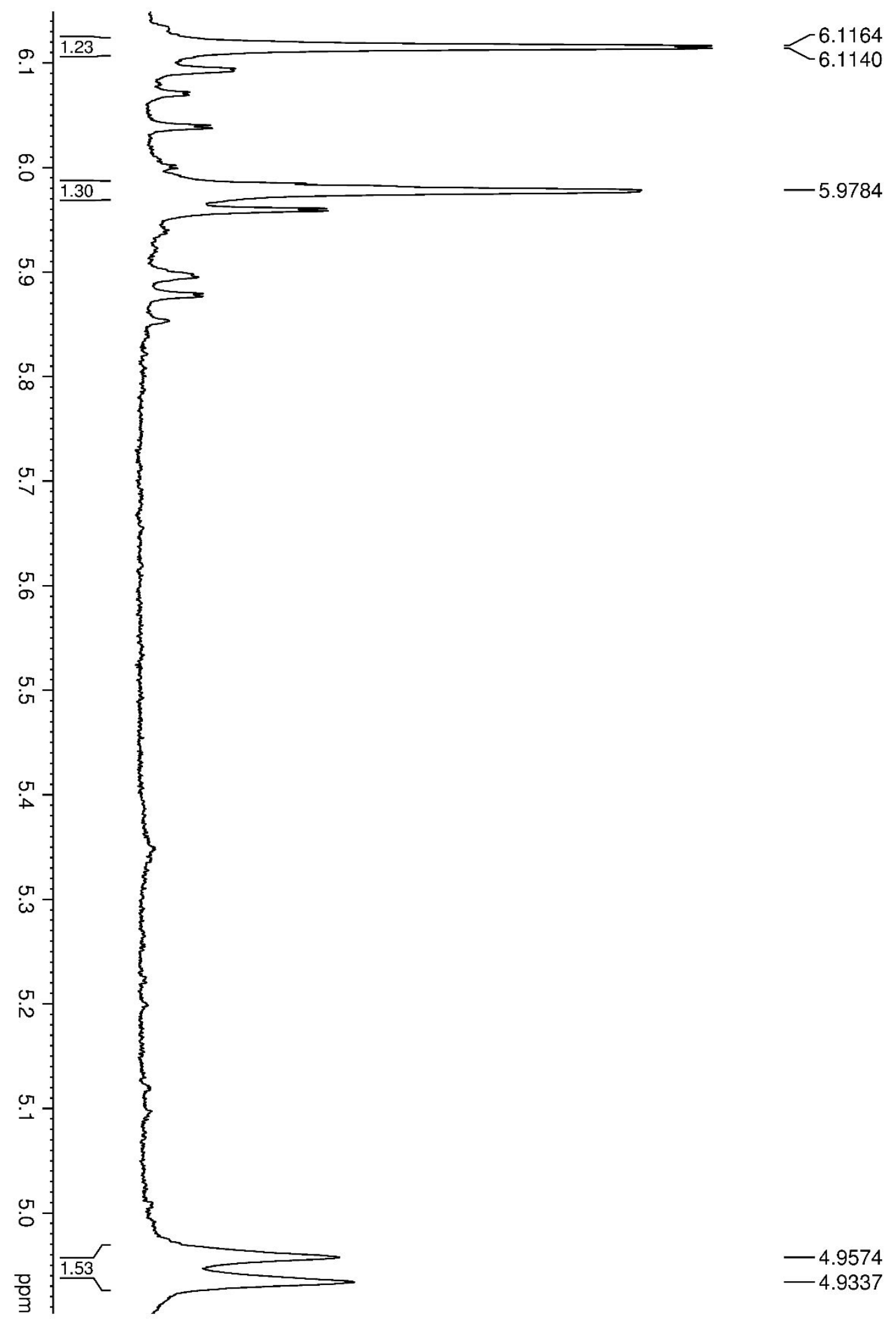


Figure S129. Enlargement ( $\delta$ 4.07-2.76) of ${ }^{1} \mathrm{H}$ NMR spectrum of compound 6 in $\mathrm{CDCl}_{3}$ at $500 \mathrm{MHz}$.

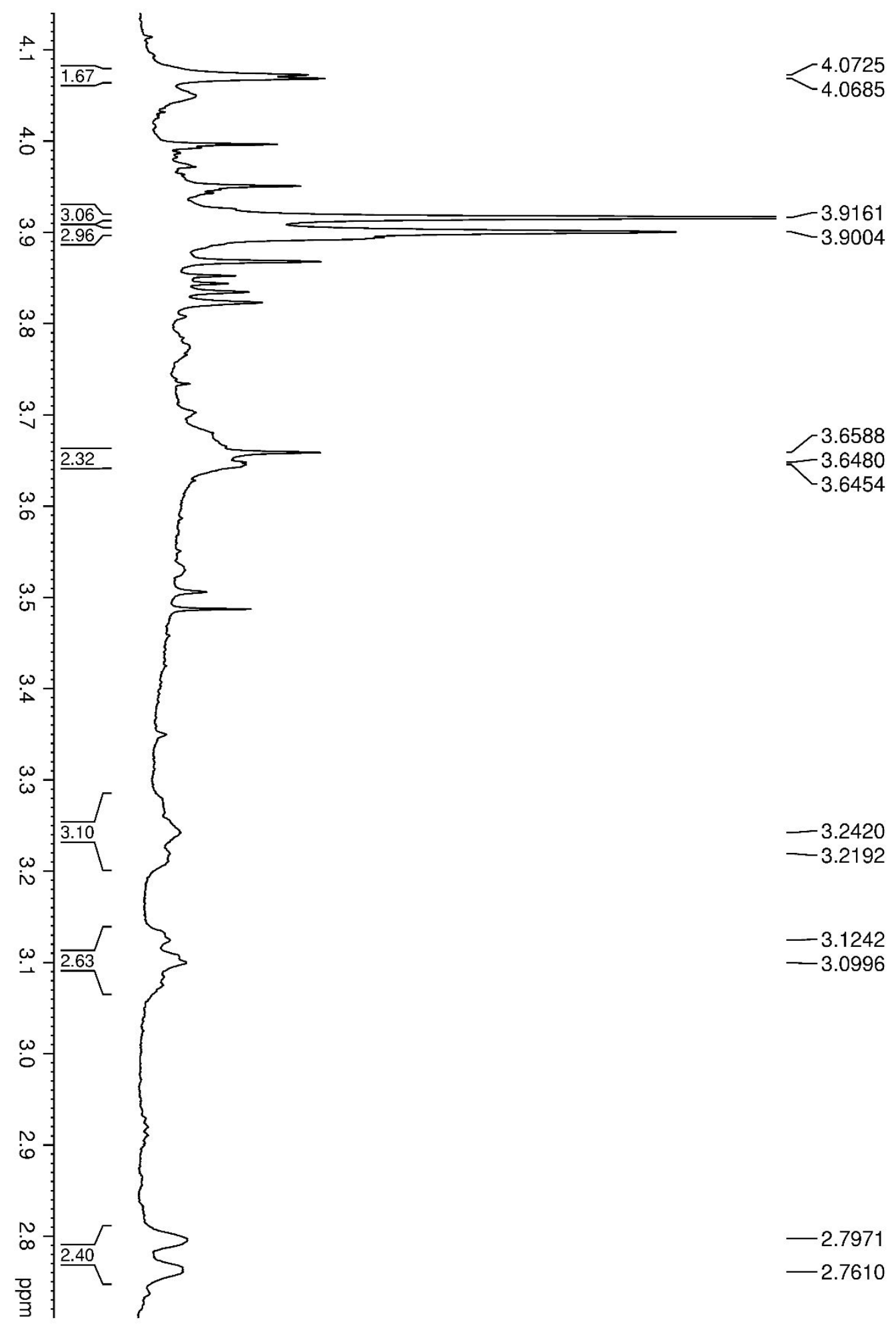


Figure S130. ${ }^{1} \mathrm{H}$ NMR spectrum of compound 7 in $\mathrm{CDCl}_{3}$ at $500 \mathrm{MHz}$.
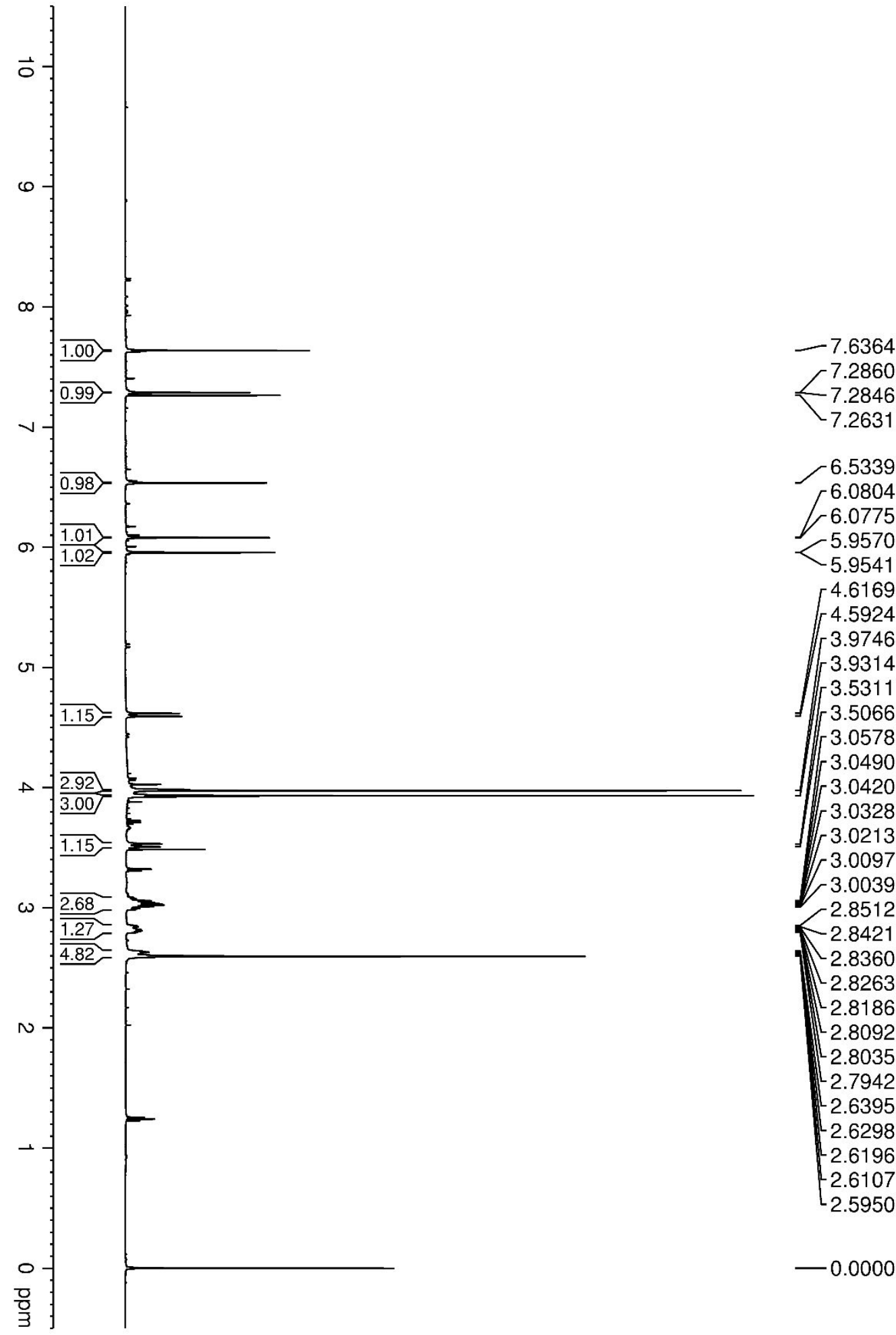
Figure S131. Enlargement ( $\delta$ 7.63-7.26) of ${ }^{1} \mathrm{H}$ NMR spectrum of compound 7 in $\mathrm{CDCl}_{3}$ at $500 \mathrm{MHz}$.

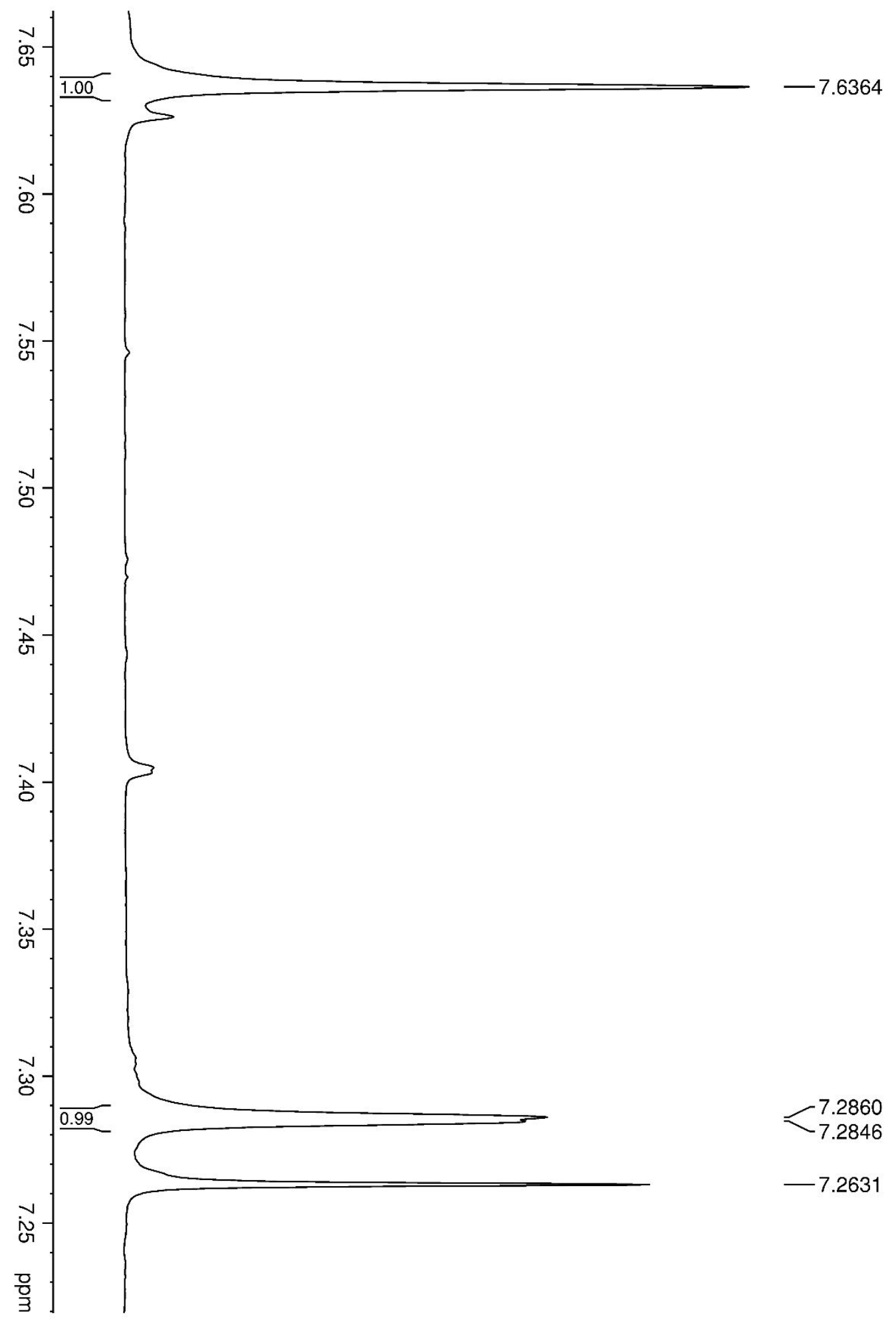


Figure S132. Enlargement ( $\delta 6.53-5.95)$ of ${ }^{1} \mathrm{H}$ NMR spectrum of compound 7 in $\mathrm{CDCl}_{3}$ at $500 \mathrm{MHz}$.

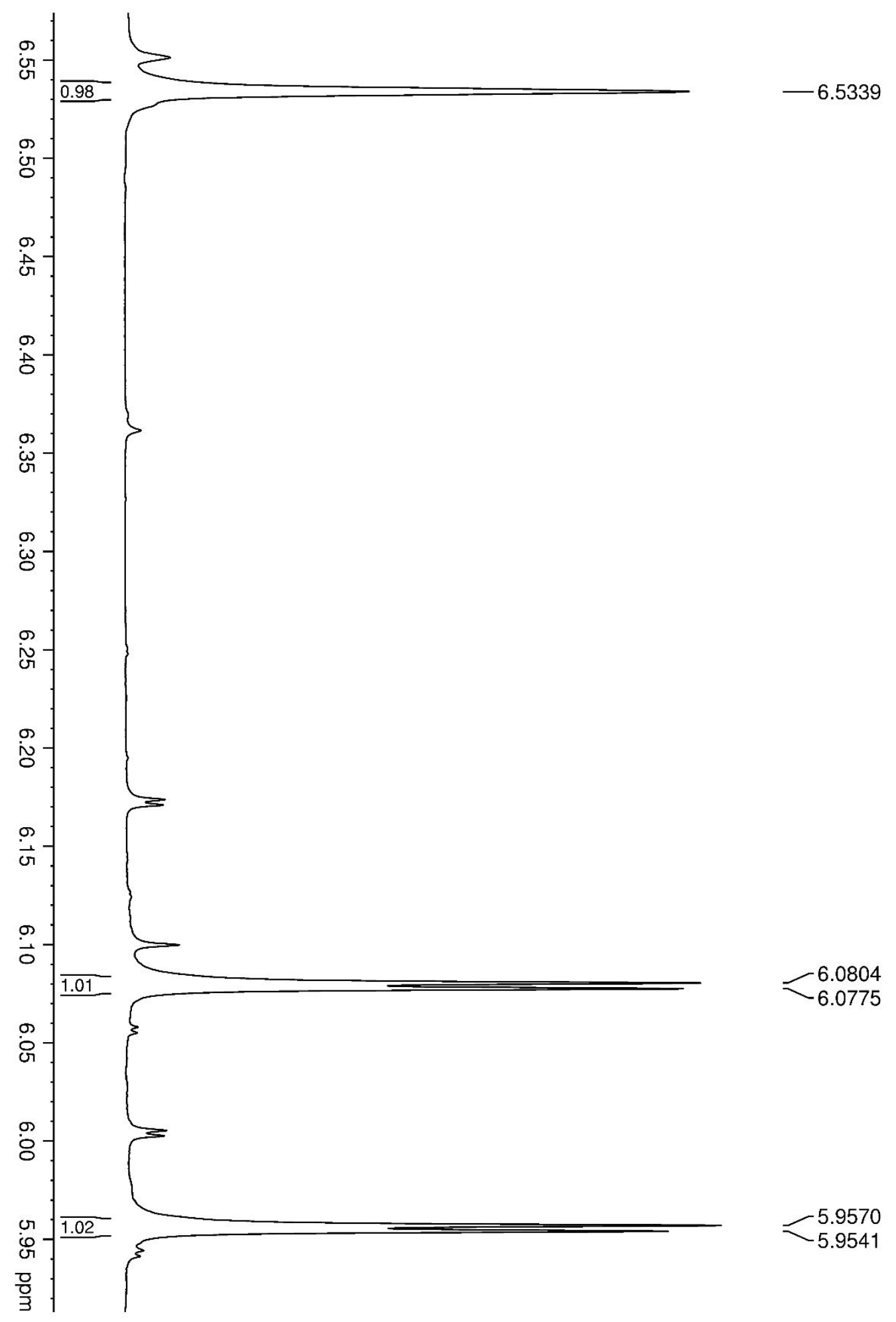


Figure S133. Enlargement ( $\delta 4.61-3.50)$ of ${ }^{1} \mathrm{H}$ NMR spectrum of compound 7 in $\mathrm{CDCl}_{3}$ at $500 \mathrm{MHz}$.

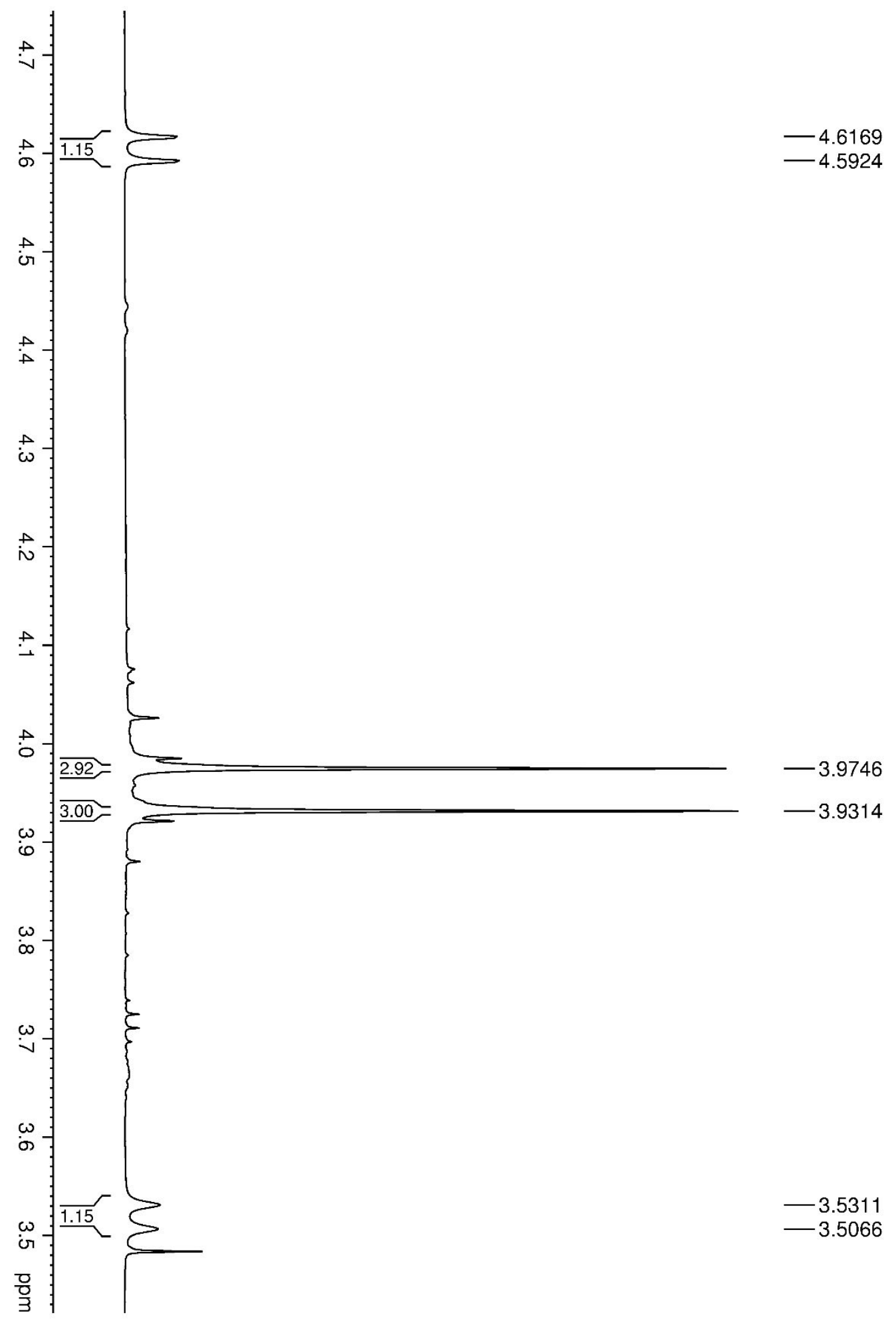


Figure S134. Enlargement ( $\delta 3.08-2.59)$ of ${ }^{1} \mathrm{H}$ NMR spectrum of compound 7 in $\mathrm{CDCl}_{3}$ at $500 \mathrm{MHz}$.

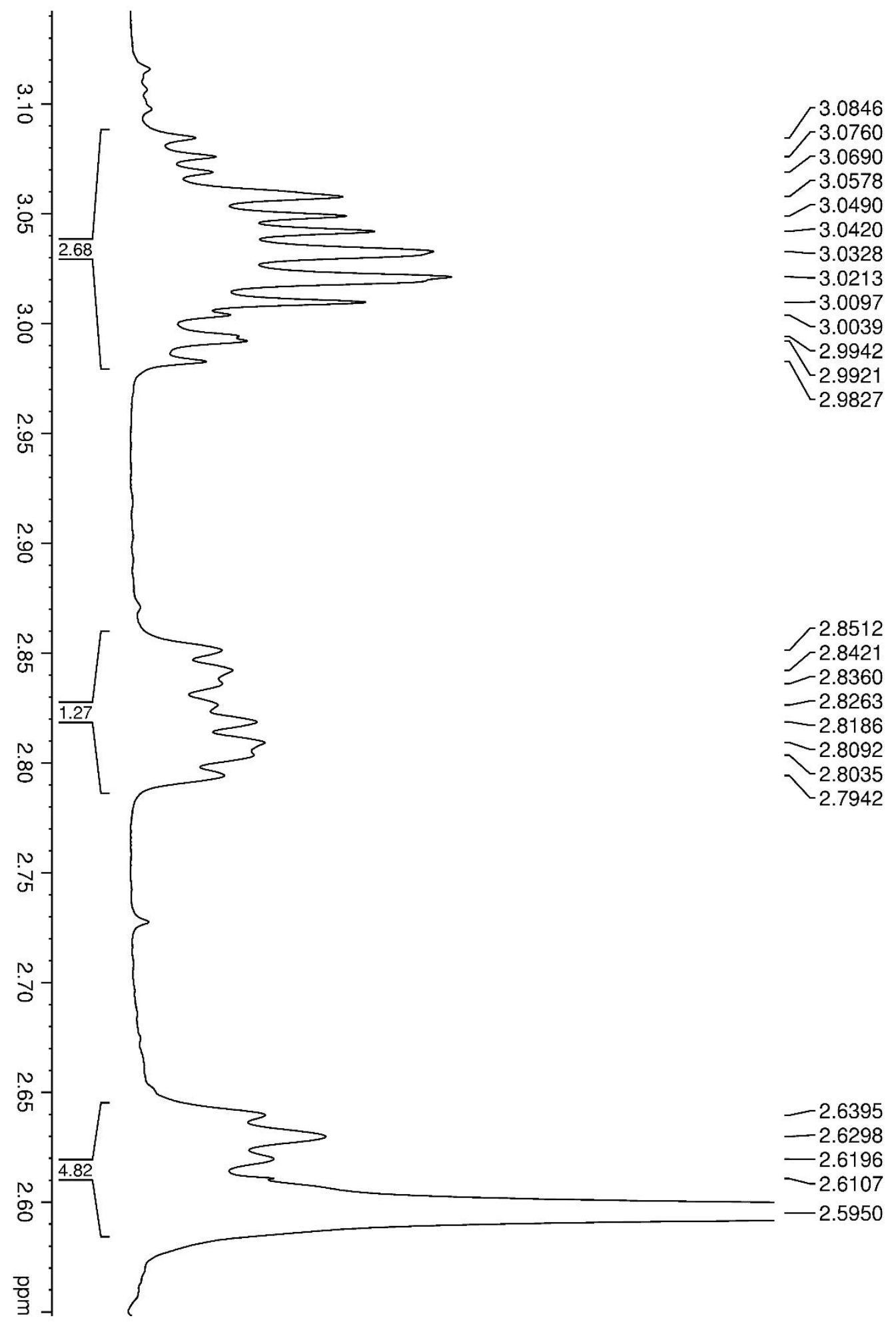


Figure S135. ${ }^{1} \mathrm{H}$ NMR spectrum of compound 8 in $\mathrm{CDCl}_{3}$ at $500 \mathrm{MHz}$.

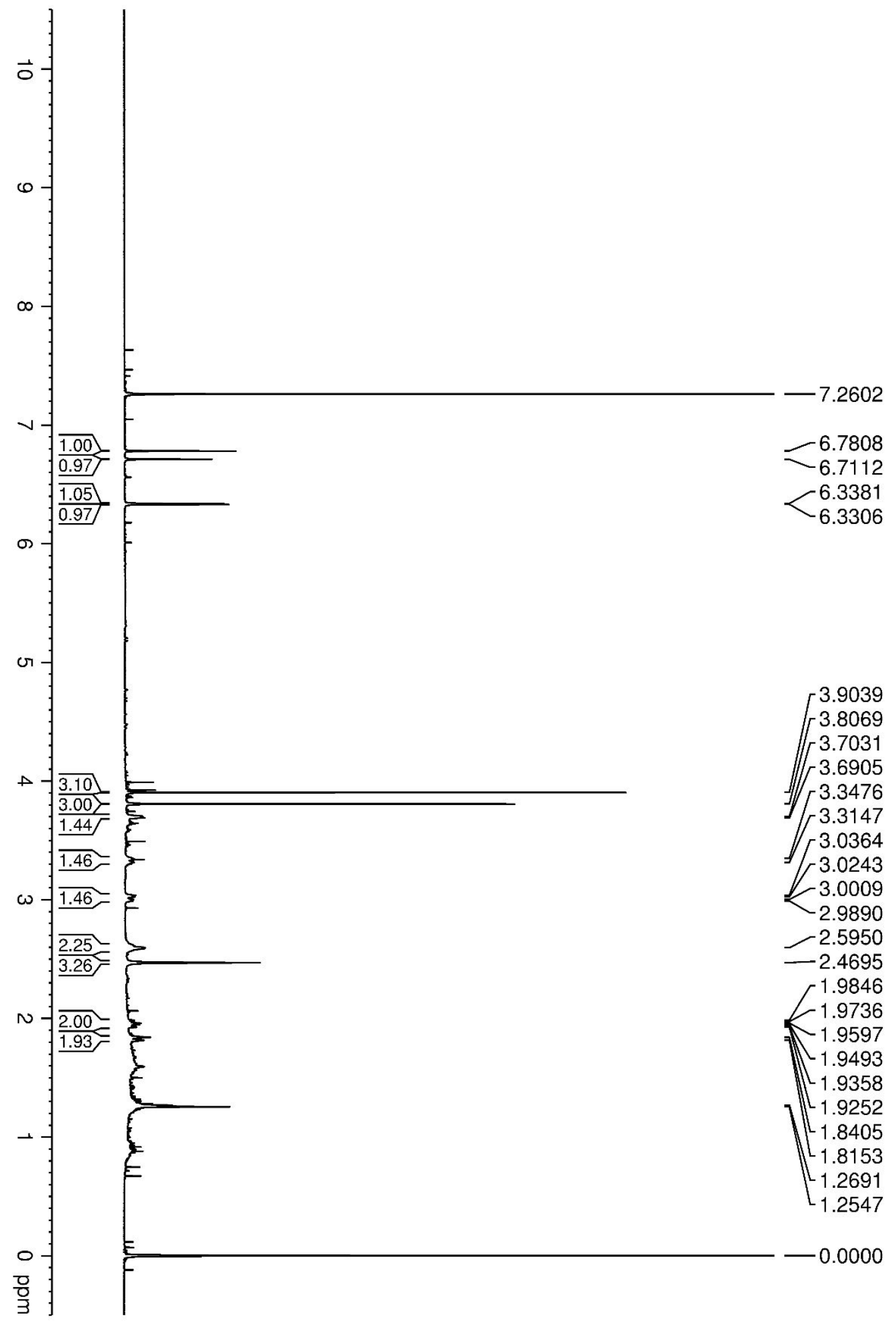


Figure S136. Enlargement ( $\delta 6.78-6.33)$ of ${ }^{1} \mathrm{H}$ NMR spectrum of compound $\mathbf{8}$ in $\mathrm{CDCl}_{3}$ at $500 \mathrm{MHz}$.

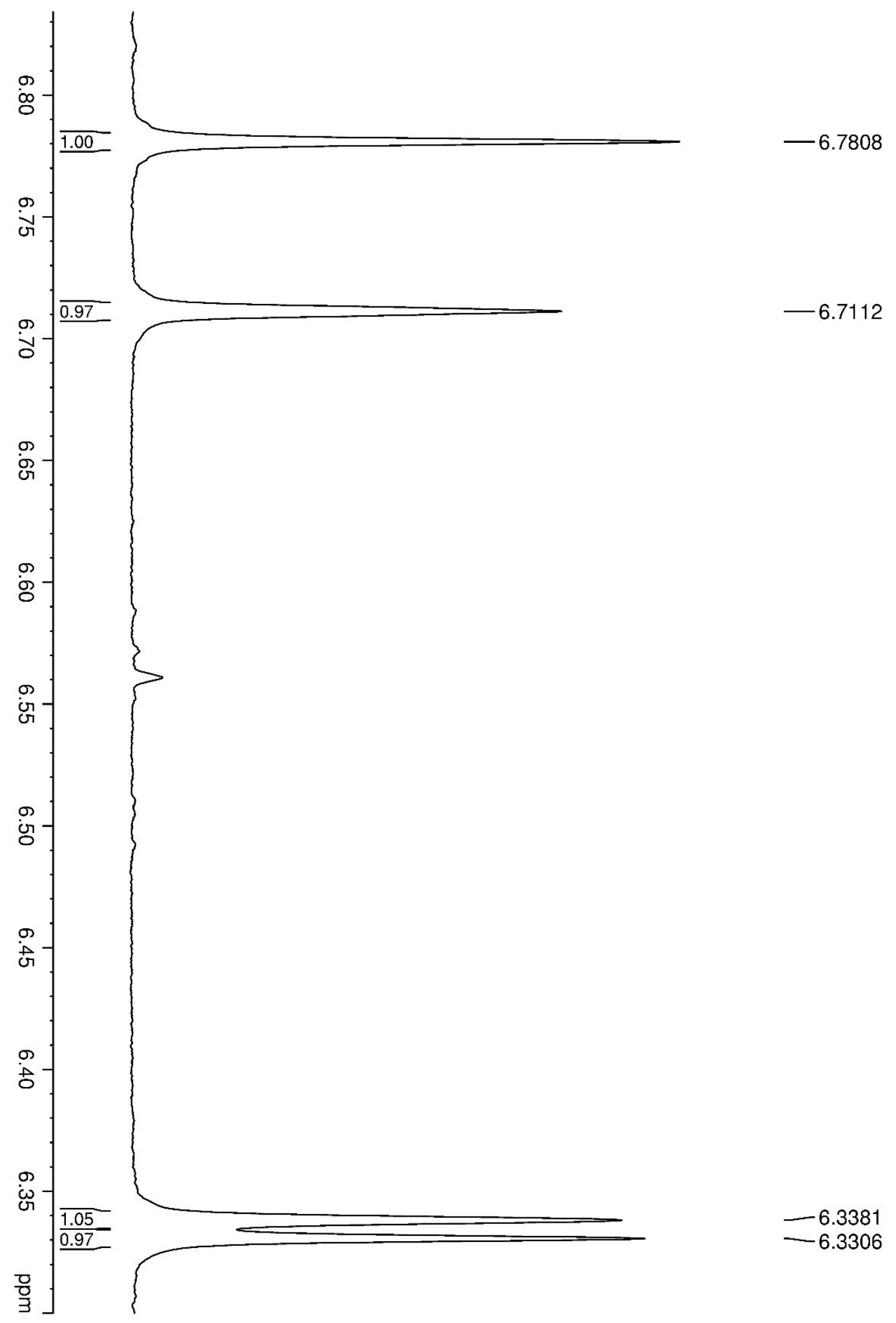


Figure S137. Enlargement ( $\delta 3.90-2.98)$ of ${ }^{1} \mathrm{H}$ NMR spectrum of compound $\mathbf{8}$ in $\mathrm{CDCl}_{3}$ at $500 \mathrm{MHz}$.

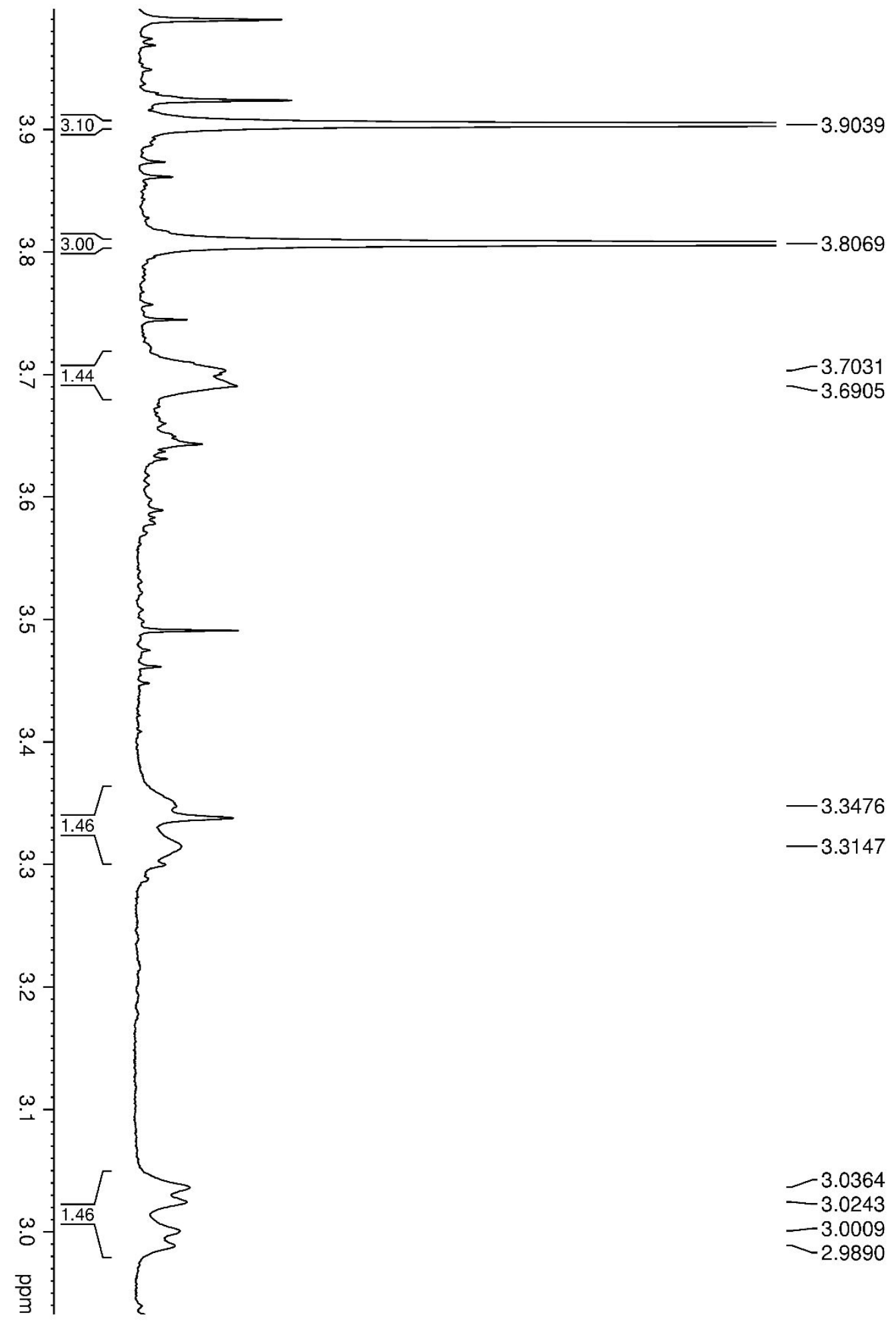


Figure S138. Enlargement ( $\delta 2.59-1.81)$ of ${ }^{1} \mathrm{H}$ NMR spectrum of compound $\mathbf{8}$ in $\mathrm{CDCl}_{3}$ at $500 \mathrm{MHz}$.

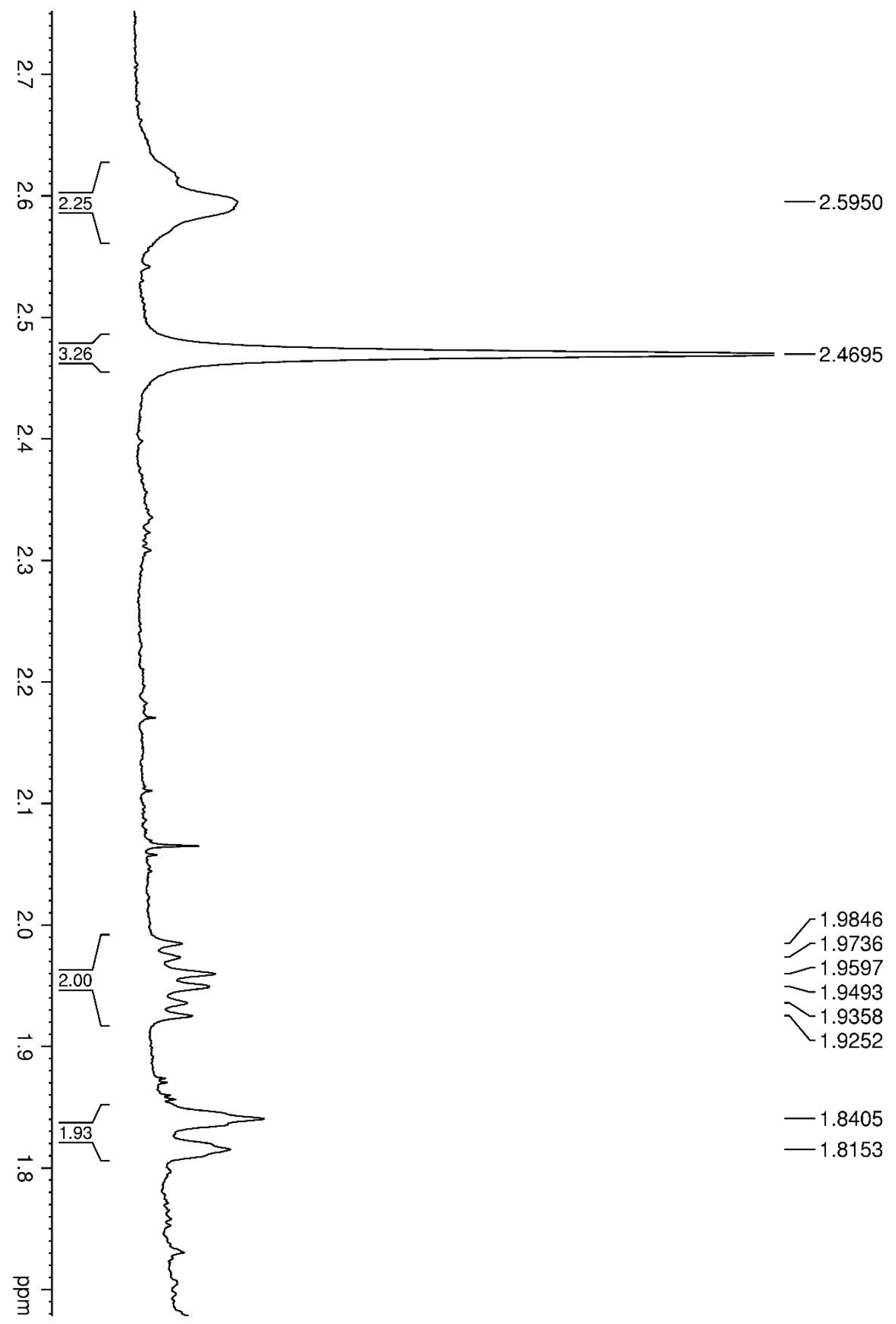


Figure S139. ${ }^{1} \mathrm{H}$ NMR spectrum of compound 9 in $\mathrm{CDCl}_{3}+$ drops of $\mathrm{CD}_{3} \mathrm{OD}$ at 300 $\mathrm{MHz}$.

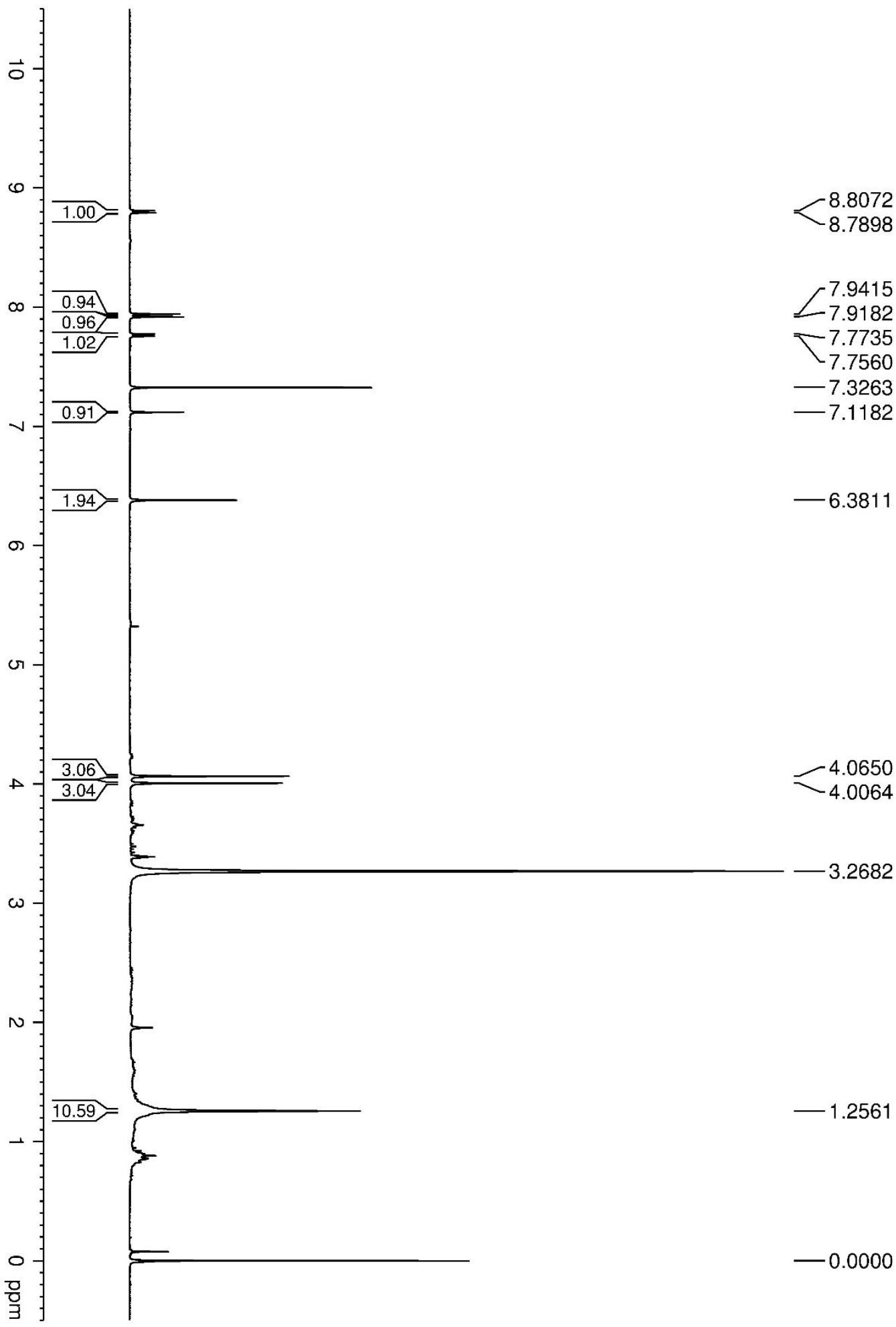


Figure S140. Enlargement ( $\delta 8.80-6.38)$ of ${ }^{1} \mathrm{H}$ NMR spectrum of compound 9 in $\mathrm{CDCl}_{3}$ + drops of $\mathrm{CD}_{3} \mathrm{OD}$ at $300 \mathrm{MHz}$.

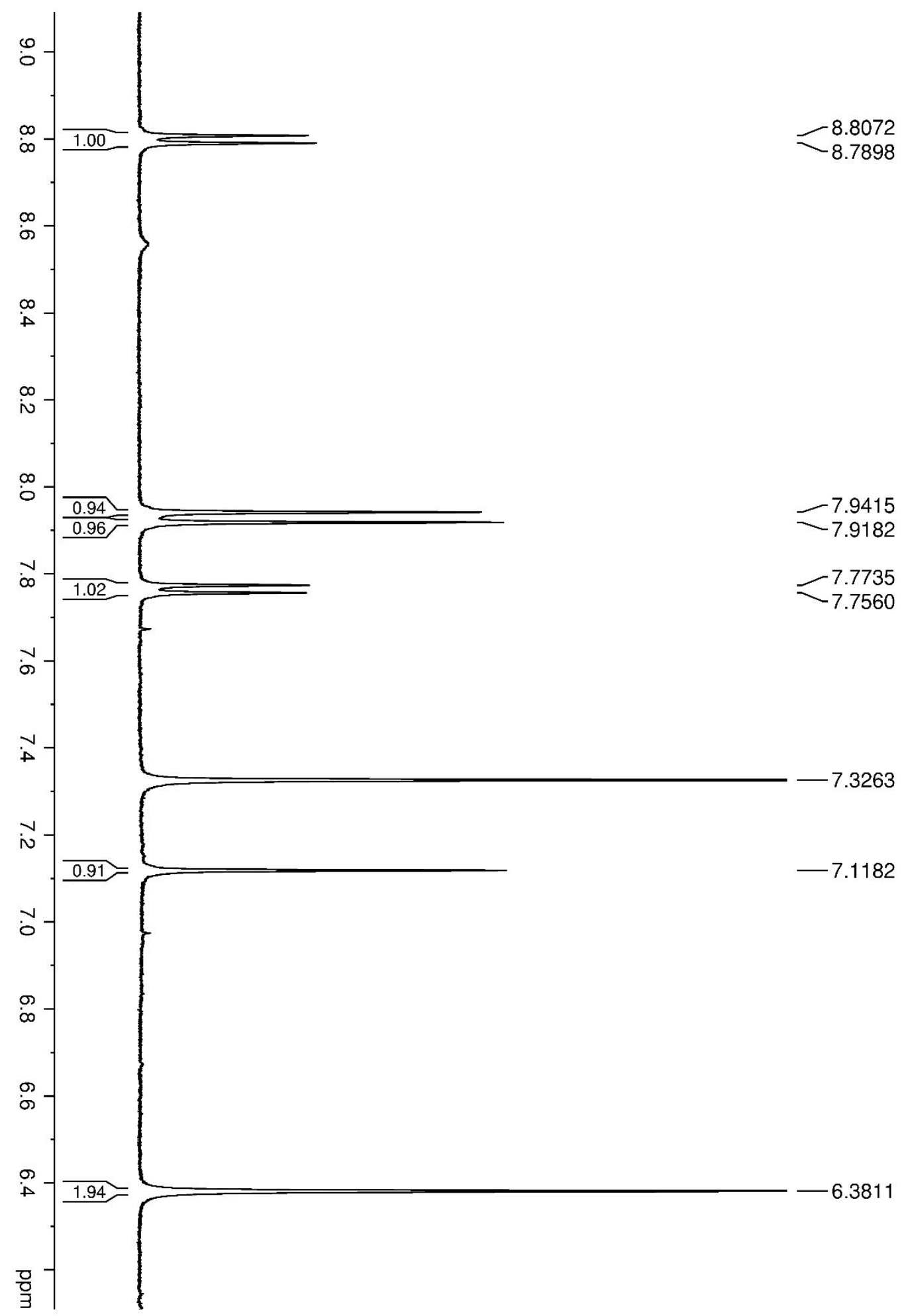


Figure S141. Enlargement ( $\delta 4.06-4.00)$ of ${ }^{1} \mathrm{H}$ NMR spectrum of compound 9 in $\mathrm{CDCl}_{3}$ + drops of $\mathrm{CD}_{3} \mathrm{OD}$ at $300 \mathrm{MHz}$.

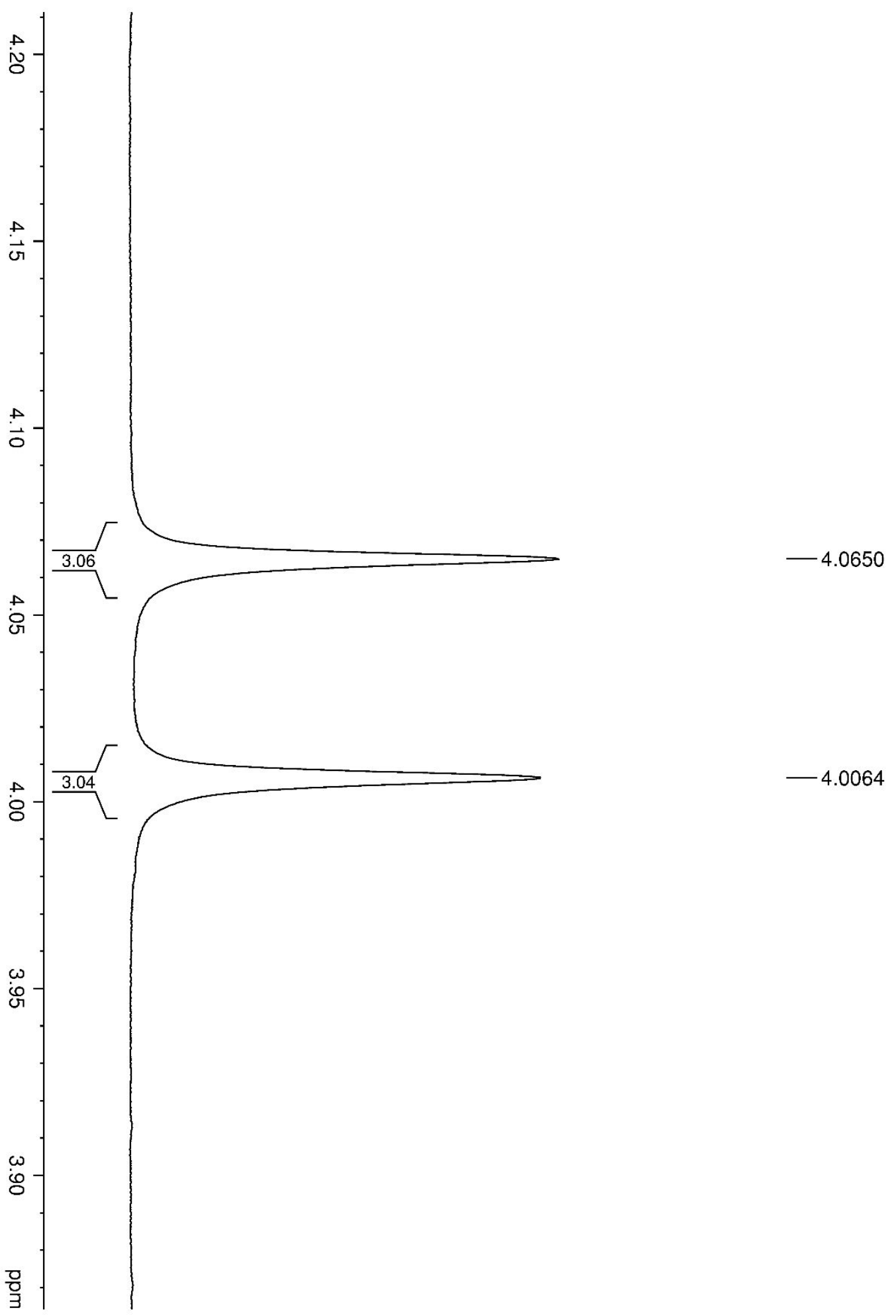


Figure S142. ${ }^{1} \mathrm{H}$ NMR spectrum of compound 11 in $\mathrm{CDCl}_{3}$ at $500 \mathrm{MHz}$.

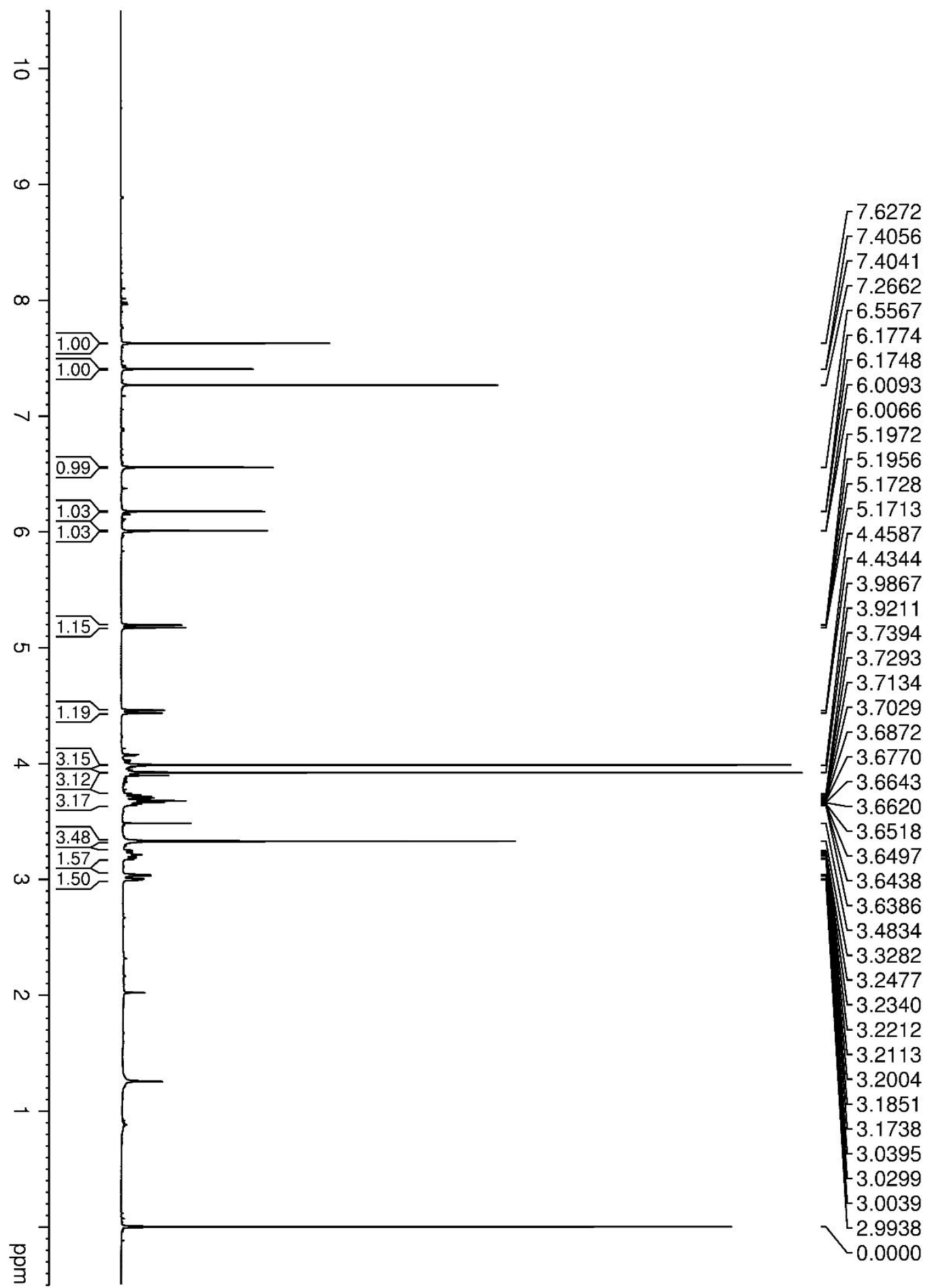


Figure S143. Enlargement ( $\delta$ 7.62-6.55) of ${ }^{1} \mathrm{H}$ NMR spectrum of compound 11 in $\mathrm{CDCl}_{3}$ at $500 \mathrm{MHz}$.

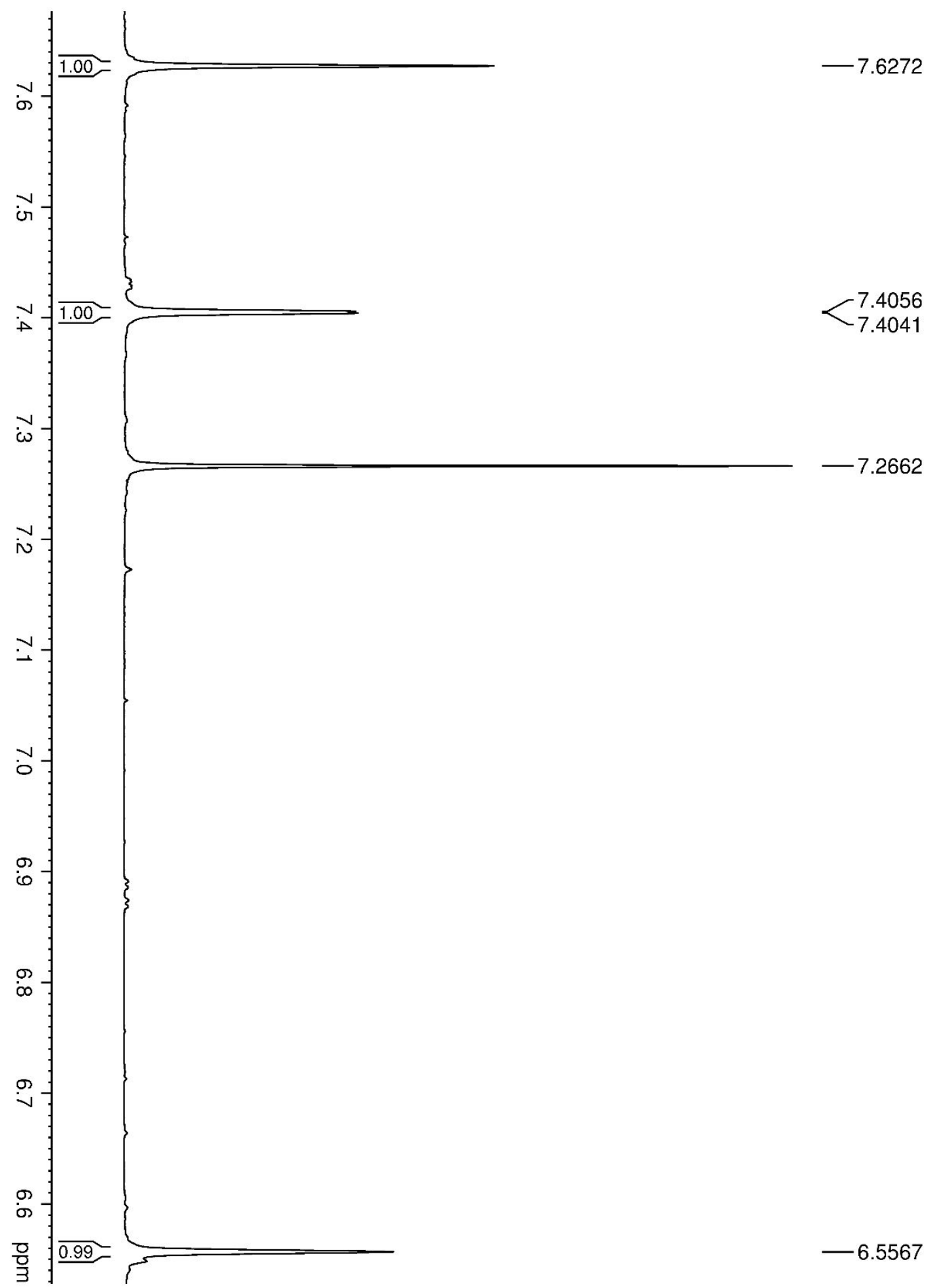


Figure S144. Enlargement ( $\delta$ 6.17-6.00) of ${ }^{1} \mathrm{H}$ NMR spectrum of compound 11 in $\mathrm{CDCl}_{3}$ at $500 \mathrm{MHz}$.

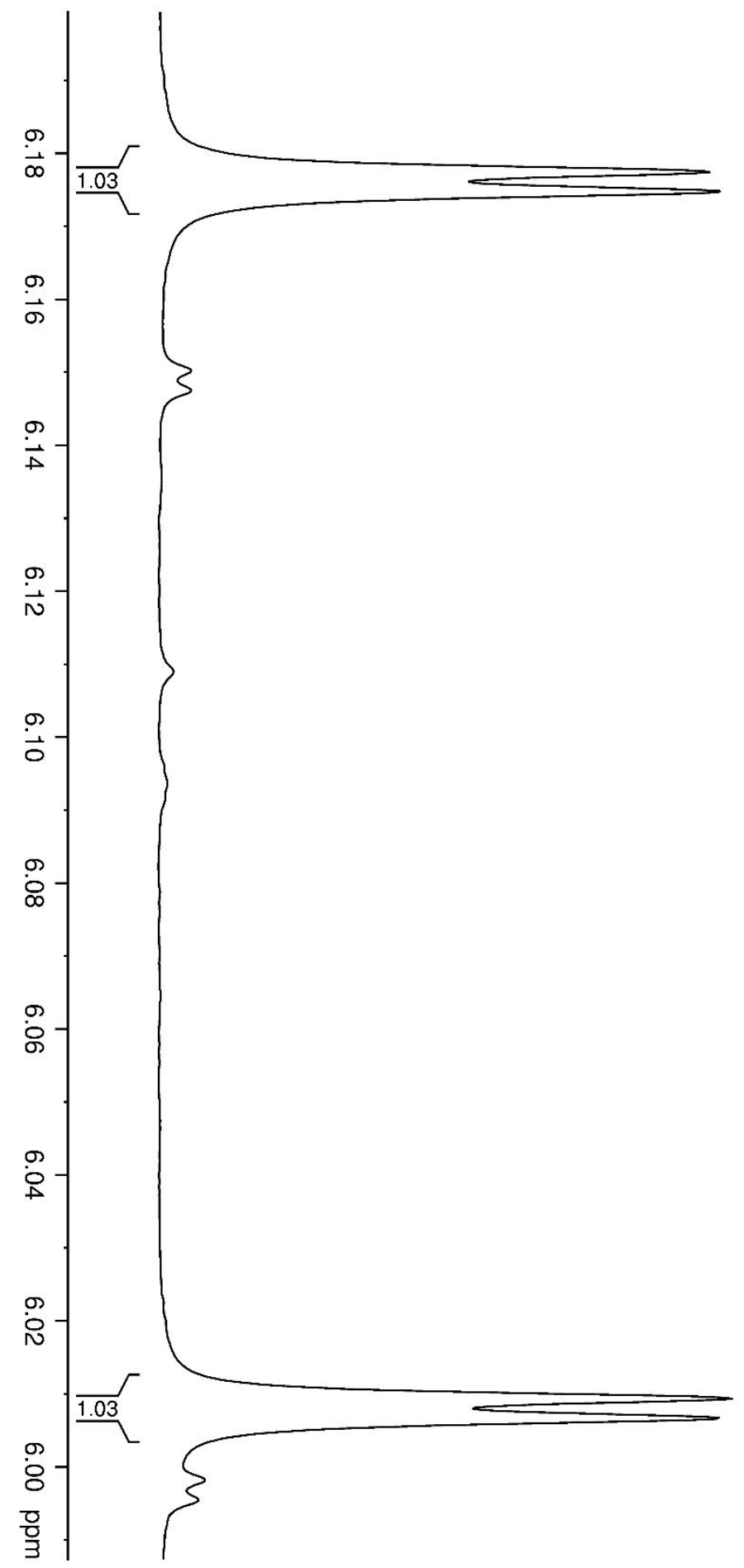


Figure S145. Enlargement ( $\delta$ 5.19-3.92) of ${ }^{1} \mathrm{H}$ NMR spectrum of compound 11 in $\mathrm{CDCl}_{3}$ at $500 \mathrm{MHz}$.

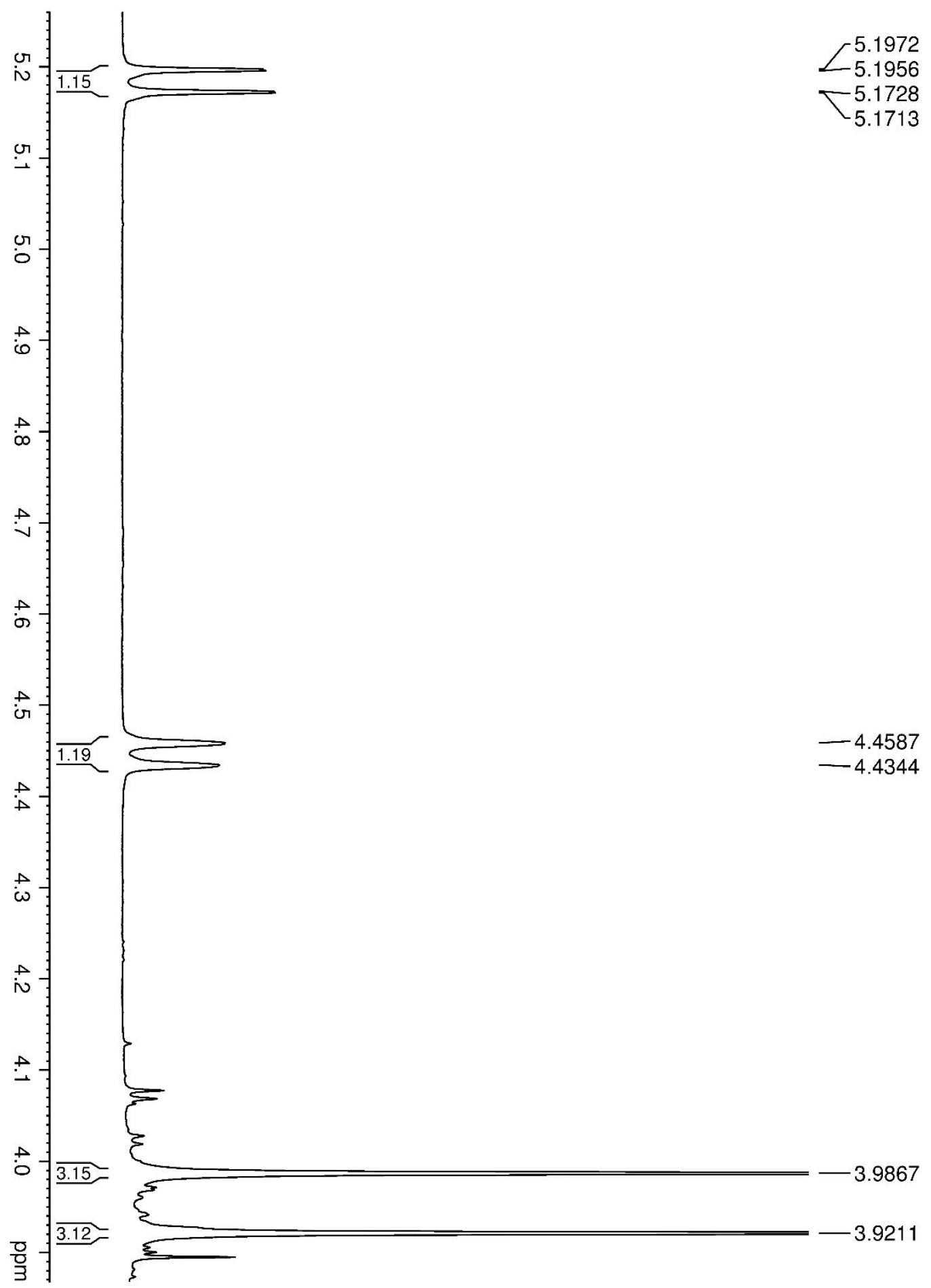


Figure S146. Enlargement ( $\delta 3.73-2.99)$ of ${ }^{1} \mathrm{H}$ NMR spectrum of compound 11 in $\mathrm{CDCl}_{3}$ at $500 \mathrm{MHz}$.

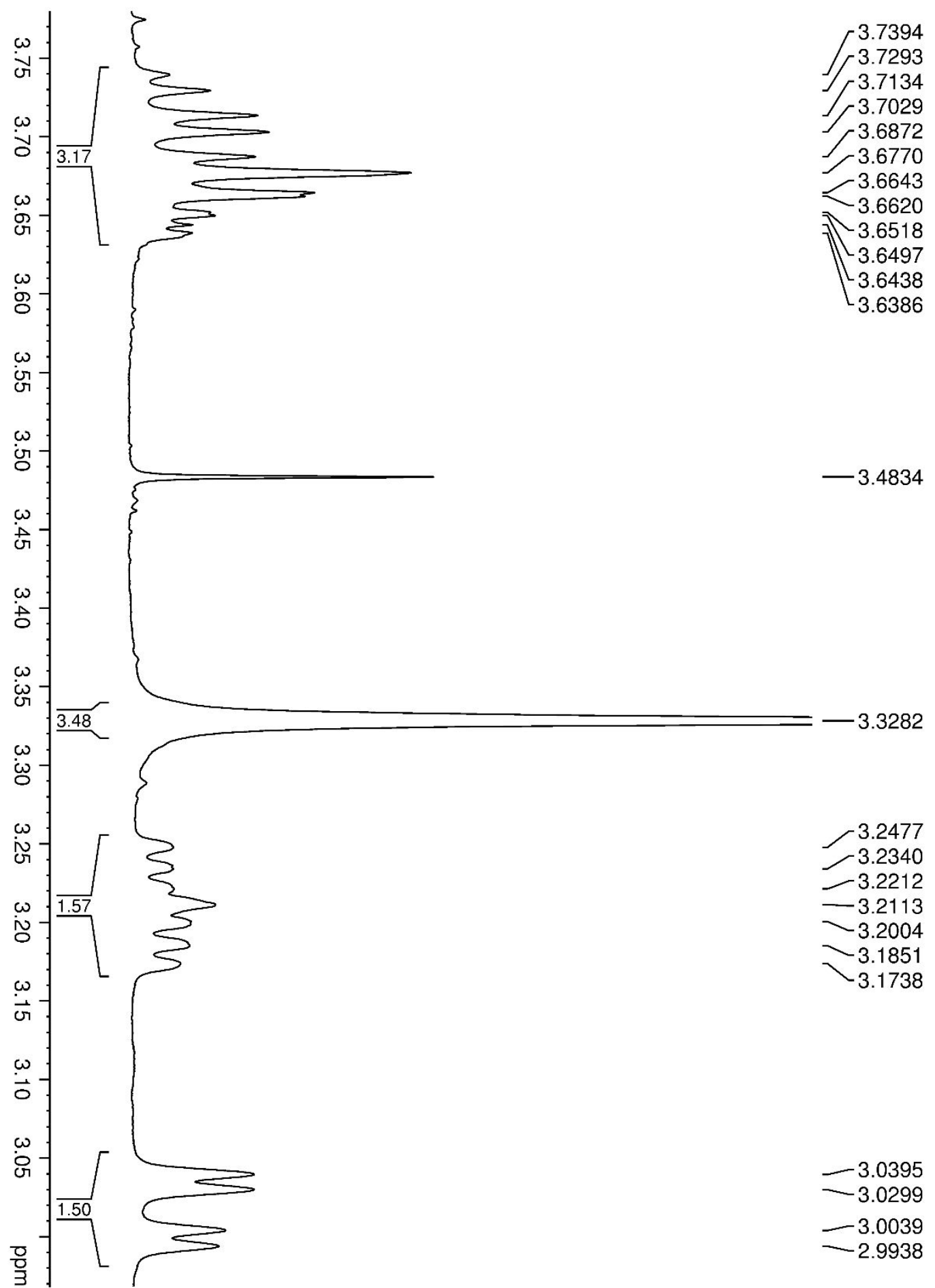




\section{Molecular geometry details of the ECD calculations}

Figure 3 - Compound R

Energy $=-1205.33806998$ hartrees

Optimized coordinates $\mathrm{x}, \mathrm{y}, \mathrm{z}$

$\begin{array}{llll}\mathrm{C} & -0.017427 & -2.310137 & -1.317671 \\ \mathrm{H} & -0.518651 & -1.877761 & -2.189620 \\ \mathrm{H} & 0.405124 & -3.270426 & -1.613843 \\ \mathrm{O} & -0.976855 & -2.570566 & -0.290950 \\ \mathrm{C} & -0.661143 & 0.422184 & -0.272118 \\ \mathrm{C} & -3.129283 & 1.863148 & -0.055055 \\ \mathrm{H} & -4.052326 & 2.415089 & 0.075304 \\ \mathrm{C} & 1.046045 & -1.417090 & -0.767486 \\ \mathrm{C} & 2.357554 & -1.874610 & -0.694416 \\ \mathrm{C} & 0.707797 & -0.183768 & -0.205869 \\ \mathrm{C} & 3.344207 & -1.142860 & -0.065949 \\ \mathrm{H} & 2.620466 & -2.837087 & -1.119565 \\ \mathrm{C} & 1.704564 & 0.528349 & 0.475278 \\ \mathrm{C} & 3.010095 & 0.066574 & 0.550591 \\ \mathrm{H} & 1.452028 & 1.453679 & 0.967417 \\ \mathrm{O} & 4.621573 & -1.642234 & 0.030568 \\ \mathrm{O} & 4.013843 & 0.707111 & 1.205626 \\ \mathrm{C} & 3.720562 & 1.940240 & 1.857227 \\ \mathrm{H} & 2.977092 & 1.802110 & 2.646081 \\ \mathrm{H} & 4.657998 & 2.272190 & 2.298913 \\ \mathrm{H} & 3.370436 & 2.688261 & 1.141877 \\ \mathrm{H} & 5.554170 & -1.078768 & -0.900176 \\ \mathrm{H} & 5.626879 & 0.003699 & -0.774684 \\ \mathrm{H} & -1.9150517 & -1.535859 & -0.687647 \\ \mathrm{H} & -256414 & -1.314069 & -1.925635 \\ \mathrm{H} & -1.766403 & -0.382973 \\ \mathrm{H} & -0.255270 & -0.173869\end{array}$




\begin{tabular}{llll}
$\mathrm{C}$ & -4.420111 & -1.736552 & 0.140368 \\
$\mathrm{C}$ & -3.108893 & 0.463378 & -0.013952 \\
$\mathrm{C}$ & -4.429514 & -0.216549 & 0.301317 \\
$\mathrm{H}$ & -5.195961 & -2.186214 & 0.762636 \\
$\mathrm{H}$ & -4.641811 & -2.004375 & -0.895850 \\
$\mathrm{H}$ & -5.221922 & 0.209185 & -0.319022 \\
$\mathrm{H}$ & -4.683209 & 0.044046 & 1.334332 \\
$\mathrm{~N}$ & -3.134509 & -2.340165 & 0.483416 \\
$\mathrm{C}$ & -2.813257 & -2.247854 & 1.904564 \\
$\mathrm{H}$ & -3.596758 & -2.750765 & 2.474348 \\
$\mathrm{H}$ & -1.871724 & -2.760384 & 2.102208 \\
$\mathrm{H}$ & -2.725424 & -1.218065 & 2.276314 \\
$\mathrm{C}$ & -1.942483 & 2.497687 & -0.276189 \\
$\mathrm{C}$ & -0.750465 & 1.803967 & -0.368271 \\
$\mathrm{C}$ & -0.372669 & 3.945047 & -0.861704 \\
$\mathrm{H}$ & 0.141067 & 4.775272 & -0.384416 \\
$\mathrm{H}$ & -0.403332 & 4.045322 & -1.951393 \\
$\mathrm{O}$ & 0.272191 & 2.720099 & -0.516059 \\
$\mathrm{O}$ & -1.704793 & 3.847154 & -0.359234 \\
$\mathrm{H}$ & -2.519139 & -1.869476 & -1.403157 \\
\hline & & & \\
$\mathrm{H}$ & &
\end{tabular}

Figure 3 - Compound S

Energy $=-1205.35405890$ hartrees

Optimized coordinates $\mathrm{x}, \mathrm{y}, \mathrm{z}$
$\begin{array}{llll}\text { C } & -0.101871 & -1.765888 & -1.707343\end{array}$
$\mathrm{H} \quad-0.610018 \quad-1.007581 \quad-2.312117$
$\mathrm{H} \quad 0.312116 \quad-2.513128 \quad-2.383233$
O $\quad-1.070560 \quad-2.464529 \quad-0.918594$
$\begin{array}{llll}\text { C } & -0.650239 & 0.563452 & -0.122545\end{array}$
$\begin{array}{llll}\text { C } & -3.300815 & 1.596029 & -0.170068\end{array}$
H $\quad-4.305738 \quad 2.000464 \quad-0.189953$ 


\begin{tabular}{|c|c|c|c|}
\hline $\mathrm{C}$ & 0.992029 & -1.128628 & -0.895149 \\
\hline $\mathrm{C}$ & 2.283723 & -1.648009 & -0.899691 \\
\hline $\mathrm{C}$ & 0.725833 & 0.016482 & -0.139547 \\
\hline $\mathrm{C}$ & 3.296960 & -1.047883 & -0.179853 \\
\hline $\mathrm{H}$ & 2.516850 & -2.535911 & -1.477328 \\
\hline $\mathrm{C}$ & 1.748051 & 0.623950 & 0.594069 \\
\hline $\mathrm{C}$ & 3.032754 & 0.099877 & 0.580878 \\
\hline $\mathrm{H}$ & 1.525605 & 1.498082 & 1.189123 \\
\hline $\mathrm{O}$ & 4.554733 & -1.603916 & -0.165400 \\
\hline $\mathrm{O}$ & 4.080579 & 0.612958 & 1.273134 \\
\hline $\mathrm{C}$ & 3.869813 & 1.787425 & 2.053405 \\
\hline $\mathrm{H}$ & 3.133832 & 1.609971 & 2.841307 \\
\hline $\mathrm{H}$ & 4.832777 & 2.018462 & 2.504239 \\
\hline $\mathrm{H}$ & 3.551046 & 2.623260 & 1.425970 \\
\hline $\mathrm{C}$ & 5.484243 & -0.995911 & -1.070741 \\
\hline $\mathrm{H}$ & 5.615400 & 0.064426 & -0.843331 \\
\hline $\mathrm{H}$ & 6.431557 & -1.516802 & -0.939448 \\
\hline $\mathrm{H}$ & 5.141453 & -1.112746 & -2.102411 \\
\hline $\mathrm{C}$ & -1.530845 & -1.772667 & 0.253737 \\
\hline $\mathrm{C}$ & -1.779425 & -0.290285 & -0.004390 \\
\hline $\mathrm{C}$ & -3.879036 & -2.165534 & -0.029562 \\
\hline $\mathrm{C}$ & -3.077028 & 0.214962 & -0.034889 \\
\hline $\mathrm{C}$ & -4.269113 & -0.700441 & 0.109329 \\
\hline $\mathrm{H}$ & -4.693480 & -2.813511 & 0.299382 \\
\hline $\mathrm{H}$ & -3.683204 & -2.398952 & -1.078510 \\
\hline $\mathrm{H}$ & -5.018641 & -0.448676 & -0.645441 \\
\hline $\mathrm{H}$ & -4.740801 & -0.520402 & 1.080983 \\
\hline $\mathrm{H}$ & -0.752354 & -1.855337 & 1.017492 \\
\hline $\mathrm{N}$ & -2.675252 & -2.504055 & 0.730900 \\
\hline $\mathrm{C}$ & -2.849445 & -2.392338 & 2.175330 \\
\hline $\mathrm{H}$ & -3.713650 & -2.987191 & 2.476599 \\
\hline
\end{tabular}




$\begin{array}{llll}\mathrm{H} & -1.969629 & -2.794638 & 2.681244 \\ \mathrm{H} & -3.004181 & -1.364154 & 2.530930 \\ \mathrm{C} & -2.205006 & 2.405897 & -0.274252 \\ \mathrm{C} & -0.913338 & 1.907275 & -0.252040 \\ \mathrm{C} & -0.815257 & 4.072592 & -0.762017 \\ \mathrm{H} & -0.479439 & 4.963638 & -0.238032 \\ \mathrm{H} & -0.760381 & 4.182860 & -1.849138 \\ \mathrm{O} & -0.024282 & 2.951705 & -0.358952 \\ \mathrm{O} & -2.162256 & 3.773046 & -0.386763\end{array}$

Molecular geometry details of the specific rotation calculations

Figure 5 - Compound R

Energy $=-804.760737768$ hartrees

Optimized coordinates $\mathrm{x}, \mathrm{y}, \mathrm{z}$

$\begin{array}{llll}\mathrm{O} & -2.316722 & -2.094520 & 0.082770 \\ \mathrm{C} & -1.423281 & -1.070368 & -0.002085 \\ \mathrm{C} & -1.955444 & 0.226931 & 0.071459 \\ \mathrm{C} & -1.110486 & 1.324703 & -0.014598 \\ \mathrm{C} & 0.264100 & 1.144294 & -0.175238 \\ \mathrm{C} & 0.802902 & -0.134885 & -0.248506 \\ \mathrm{C} & -0.057584 & -1.228299 & -0.158043 \\ \mathrm{O} & -3.299952 & 0.316030 & 0.222057 \\ \mathrm{H} & -1.521084 & 2.320319 & 0.042906 \\ \mathrm{O} & 1.145842 & 2.184184 & -0.261581 \\ \mathrm{C} & 2.289862 & -0.328797 & -0.403765 \\ \mathrm{H} & 0.373280 & -2.215583 & -0.234081 \\ \mathrm{C} & -3.884424 & 1.604621 & 0.313839 \\ \mathrm{H} & -4.953303 & 1.439561 & 0.435208 \\ \mathrm{H} & -3.504907 & 2.152566 & 1.181366 \\ \mathrm{H} & -3.712450 & 2.187483 & -0.595793 \\ \mathrm{C} & -1.818985 & -3.417045 & -0.022544\end{array}$




$\begin{array}{llll}\mathrm{H} & -2.685602 & -4.070827 & 0.059119 \\ \mathrm{H} & -1.330310 & -3.585422 & -0.986984 \\ \mathrm{H} & -1.118694 & -3.647328 & 0.786249 \\ \mathrm{C} & 0.639343 & 3.506547 & -0.330819 \\ \mathrm{H} & -0.026957 & 3.634434 & -1.189061 \\ \mathrm{H} & 0.114247 & 3.784120 & 0.587993 \\ \mathrm{H} & 1.506251 & 4.153495 & -0.452887 \\ \mathrm{O} & 2.544645 & -1.621639 & -0.940809 \\ \mathrm{H} & 3.494738 & -1.771094 & -0.857970 \\ \mathrm{C} & 3.012480 & -0.179057 & 0.924380 \\ \mathrm{H} & 2.843457 & 0.822576 & 1.328579 \\ \mathrm{H} & 2.634576 & -0.925522 & 1.630997 \\ \mathrm{O} & 4.399614 & -0.396179 & 0.663715 \\ \mathrm{H} & 4.867489 & -0.506911 & 1.497348 \\ \mathrm{H} & 2.680948 & 0.433256 & -1.086676\end{array}$

Figure 5 - Compound S

Energy $=-804.760737766$ hartrees

Optimized coordinates $\mathrm{x}, \mathrm{y}, \mathrm{z}$
$\begin{array}{llll}\text { O } & 2.316457 & -2.094672 & 0.082858\end{array}$
$\begin{array}{llll}\text { C } & 1.423105 & -1.070459 & -0.002100\end{array}$
$\begin{array}{llll}\text { C } & 1.955431 & 0.226820 & 0.071294\end{array}$
$\begin{array}{llll}\text { C } & 1.110599 & 1.324659 & -0.014914\end{array}$
$\begin{array}{llll}\text { C } & -0.264035 & 1.144362 & -0.175484\end{array}$
$\begin{array}{llll}\text { C } & -0.802964 & -0.134737 & -0.248626\end{array}$
$\begin{array}{llll}\text { C } & 0.057415 & -1.228250 & -0.158067\end{array}$
$\begin{array}{llll}\text { O } & 3.299944 & 0.315755 & 0.221800\end{array}$
$\begin{array}{llll}\mathrm{H} & 1.521275 & 2.320259 & 0.042321\end{array}$
O $\quad-1.145618 \quad 2.184379 \quad-0.262032$
C $\quad-2.289882 \quad-0.328629 \quad-0.403778$
$\begin{array}{llll}\mathrm{H} & -0.373642 & -2.215467 & -0.234003\end{array}$ 


$\begin{array}{llll}\mathrm{C} & 3.884522 & 1.604277 & 0.314095 \\ \mathrm{H} & 4.953291 & 1.439065 & 0.436225 \\ \mathrm{H} & 3.713211 & 2.187197 & -0.595622 \\ \mathrm{H} & 3.504495 & 2.152191 & 1.181413 \\ \mathrm{C} & 1.818587 & -3.417175 & -0.022409 \\ \mathrm{H} & 2.685145 & -4.071024 & 0.059348 \\ \mathrm{H} & 1.118234 & -3.647333 & 0.786362 \\ \mathrm{H} & 1.329960 & -3.585533 & -0.986872 \\ \mathrm{C} & -0.638906 & 3.506685 & -0.330282 \\ \mathrm{H} & 0.027653 & 3.635049 & -1.188255 \\ \mathrm{H} & -1.505674 & 4.153851 & -0.452224 \\ \mathrm{H} & -0.114025 & 3.783589 & 0.588858 \\ \mathrm{H} & -2.681067 & 0.433502 & -1.086583 \\ \mathrm{C} & -3.012375 & -0.178911 & 0.924412 \\ \mathrm{H} & -2.634360 & -0.925204 & 1.631170 \\ \mathrm{H} & -2.843444 & 0.822763 & 1.328558 \\ \mathrm{O} & -4.399501 & -0.396229 & 0.663861 \\ \mathrm{H} & -4.867429 & -0.506553 & 1.497521 \\ \mathrm{O} & -2.544749 & -1.621413 & -0.940864 \\ \mathrm{H} & -3.494862 & -1.770768 & -0.858056\end{array}$

